\title{
A study of $B_{s}^{0} \rightarrow J / \psi \phi$ in the $\mathrm{D} \varnothing$ experiment and an example of HEP technology transfer
}

\section{Daniela Ursula Bauer}

\author{
Imperial College of Science, Technology and Medicine
}

A thesis submitted for the degree of
Doctor of Philosophy
of The University of London
and the Diploma of Imperial College.

August, 2002 


\title{
A study of $B_{s}^{0} \rightarrow J / \psi \phi$ in the $\mathrm{D} \varnothing$ experiment and an example of HEP technology transfer
}

\author{
Daniela Ursula Bauer \\ Imperial College of Science, Technology and Medicine
}

August, 2002

\begin{abstract}
After years of preparation, data taking with the upgraded $\mathrm{D} \emptyset$ detector at the Tevatron protonantiproton collider has begun. The large amount of data produced in a $p \bar{p}$-collider requires sophisticated triggers to filter out the interesting events. Described in this thesis is the development of trigger software for the newly implemented Silicon Microstrip Tracker.

$\mathrm{D} \emptyset$ is a multi-purpose detector with a broad physics programme. One area being studied at $\mathrm{D} \emptyset$ is $B$ mesons. An algorithm for reconstructing the $B_{s}^{0}$ and $B_{d}^{0}$ mesons and for measuring their lifetimes has been developed and is described in this thesis. The results suggest that an improvement of the current lifetime measurements can be achieved within the next two years.

The reconstruction of a $J / \psi$ meson forms the basis for a wide range of $b$-physics. Data taken with the muon system during the commissioning period of the detector has been analysed and a signal for the $J / \psi$ meson has been found.

Systematic transfer of HEP technologies into other areas and their commercial exploitation will play an important role in the future of particle physics. An area of particular interest is DNA sequencing as shown by the recent completion of the sequencing of the human genome. The final part of this thesis details the development of a simulation for a high throughput sequencing device which is currently being developed at Imperial College.
\end{abstract}




\section{Acknowledgements}

I would like to thank ....

John Hassard for finding the funding for this $\mathrm{PhD}$ and making it all possible.

Gavin Davies for the encouragement and for the help in dealing with the authorities. He is just so much more diplomatic than I could ever be.

Gavin Davies, Vivek Jain, Ray Beuselinck, John Hassard and Trevor Bacon for reading this thesis, correcting spelling and grammar, offering insights and hints and for generally being helpful.

My colleagues Robert Illingworth and Ray Beuselinck for helping me get started with $\mathrm{D} \varnothing$ software and Robert for explaining the finer points of the SMT to me. Vivek Jain, Kin Yip, Wendy Taylor and Farrukh Azfar for dealing with the pitfalls of $b$-physics, Monte-Carlo data or both.

Ray Beuselinck for sorting out countless software problems, his special sense of humour, letting me use his fridge, driving my stuff all the way to Whitechapel and for extracting my laptop from the claws of Dell.

On a different note, I'd like to thank my fellow graduate students/post-docs at FNAL (especially Lucio, Freya and Greg) and at Imperial (especially Richard, Rod, Robert, Barry and Ricardo) for all the entertainment they provided. Michele would have been an almost ideal cubicle mate at FNAL, if it wasn't for those endless conversations with his girlfriend.

I like to thank Jonathan Hays $\odot$ for those interesting times. I would have enjoyed the last 3 years a lot less without him.

I acknowledge that PPARC paid the fees for this $\mathrm{PhD}$. 


\section{Contents}

$\begin{array}{ll}\text { Abstract } & 2\end{array}$

Acknowledgements $\quad 3$

$\begin{array}{ll}\text { Contents } & 4\end{array}$

$\begin{array}{lr}\text { List of Figures } & 8\end{array}$

List of Tables $\quad 12$

Chapter 1. Introduction $\quad 13$

1.1 The Standard Model 13

1.2 Triggering and reconstruction software 14

1.3 Technology transfer $\quad 15$

Chapter 2. $B$-mesons: CP-violation, mixing and lifetimes 16

2.1 Symmetries in the Standard Model 16

2.2 The discovery of CP-violation 18

$\begin{array}{lll}2.3 & \text { The CKM matrix } & 19\end{array}$

2.4 Mixing in the neutral B-meson system 22

2.5 Types of CP-violation 25

2.6 Lifetimes 27

2.7 The decay $B_{s}^{0} \rightarrow J / \psi \phi$

2.8 The decay $B_{d}^{0} \rightarrow J / \psi K^{* 0}$

$2.9 B$-meson production at the Tevatron 34 
$\begin{array}{lll}\text { Chapter 3. } & \text { The DØ Detector at the Tevatron } & 36\end{array}$

3.1 The Tevatron at Fermilab 36

3.2 The DØ Detector 36

$\begin{array}{lll}3.2 .1 & \text { Coordinate System } & 39\end{array}$

3.2.2 Central Tracking System 39

3.2.3 Solenoid Magnet 43

3.2.4 Preshower Detectors 43

3.2.5 Calorimeter 44

3.2.6 Intercryostat Detector 45

$\begin{array}{lll}3.2 .7 & \text { Muon System } & 46\end{array}$

$\begin{array}{lll}3.3 & \text { Trigger } & 47\end{array}$

3.3.1 Level 1 and Level 2 Trigger 47

3.3.2 Level 3 Trigger 48

3.4 Software 49

Chapter 4. Clustering for the Silicon Microstrip Tracker 51

4.1 SMT clustering for the Level 3 trigger $\quad 51$

4.1.1 Coordinate systems $\quad 52$

4.1.2 Two-dimensional clustering $\quad 54$

4.1.3 Three-dimensional clustering 56

$\begin{array}{lll}4.2 & \text { Evaluation of the software } & 58\end{array}$

4.2.1 Monte Carlo matching $\quad 58$

4.2.2 Results: Resolutions, efficiencies and timing 59

4.3 Calibration 63

$\begin{array}{lll}4.4 \text { Cosmics } & 67\end{array}$

$\begin{array}{ll}\text { Chapter 5. Analysis } & 73\end{array}$

$\begin{array}{lll}5.1 & \text { Introduction } & 73\end{array}$

5.2 The Monte Carlo data sample $\quad 74$

$\begin{array}{lll}5.2 .1 & \text { Software } & 74\end{array}$

$\begin{array}{lll}5.2 .2 & \text { Signal sample } & 75\end{array}$ 
$\begin{array}{lll}\text { 5.2.3 Background sample } & 76\end{array}$

5.3 The reconstruction of $B_{s}^{0} \rightarrow J / \psi \phi$

$\begin{array}{lll}\text { 5.3.1 Muon reconstruction } & 78\end{array}$

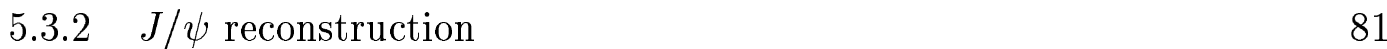

5.3.3 $\phi$ reconstruction 84

5.3.4 $B_{s}^{0}$ reconstruction $\quad 86$

$\begin{array}{lll}5.3 .5 & \text { Vertex reconstruction } & 89\end{array}$

$\begin{array}{lll}\text { 5.3.6 Lifetime } & 94\end{array}$

$\begin{array}{llr}5.3 .7 & \text { Fitting } & 97\end{array}$

5.4 The reconstruction of $B_{d}^{0} \rightarrow J / \psi K^{* 0} \quad 100$

$\begin{array}{lll}5.4 .1 & \text { Lifetime } & 105\end{array}$

$\begin{array}{lll}5.5 & \text { Cross-talk } & 108\end{array}$

$\begin{array}{lll}5.6 & \text { Event yields } & 108\end{array}$

5.7 Angular distributions $\quad 110$

$\begin{array}{lll}5.7 .1 & \text { Generators for } b \text {-physics } & 110\end{array}$

5.7.2 Shaping angular distributions 113

$5.8 \mathrm{~J} / \psi$ reconstruction using real data $\quad 114$

$\begin{array}{lll}\text { Chapter 6. Technology transfer } & 118\end{array}$

$\begin{array}{lll}6.1 & \text { Introduction } & 118\end{array}$

$\begin{array}{lll}6.2 & \text { DNA sequencing } & 119\end{array}$

$\begin{array}{lll}\text { 6.2.1 The DNA helix } & 119\end{array}$

6.2.2 Traditional DNA sequencing 119

$\begin{array}{lll}\text { 6.2.3 Capillary electrophoresis } & 122\end{array}$

6.2.4 Lambert-Beer's Law 123

6.3 The $\mu$ DiaGene Project 124

6.3.1 Efficiency of capillary electrophoresis 124

6.3.2 Status of the $\mu$ DiaGene project 125

6.3.3 Algorithms developed for $\mu$ DiaGene 126

6.4 MEDUSA: A simulation tool for $\mu$ DiaGene 128 
$\begin{array}{lll}\text { 6.4.1 MEDUSA Overview } & 128\end{array}$

$\begin{array}{ll}\text { 6.4.2 The main program } & 129\end{array}$

$\begin{array}{lll}6.4 .3 & \text { Algorithm } & 130\end{array}$

6.4.4 Input data 131

$\begin{array}{lll}\text { 6.4.5 Results } & 134\end{array}$

$\begin{array}{lll}\text { Chapter 7. Conclusion } & 136\end{array}$

7.1 The DØ Run II b-physics programme 136

$\begin{array}{lll}7.2 & \text { Summary and Outlook } & 139\end{array}$

Appendix A. $\quad 142$

A.1 Lorentz transformations 142

A.2 A cookbook recipe for calculating the transversity angle $\cos \theta_{t} \quad 143$

A.3 Error on the reconstructed proper decay length 143

A.4 Straight line fit: Method of the Least Squares 144

A.5 The Method of Maximum Likelihood 146

$\begin{array}{ll}\text { References } & 151\end{array}$ 


\section{List of Figures}

2.1 Two unitarity triangles of the CKM matrix.

2.2 Box diagrams for $B_{s}^{0} \leftrightarrow \bar{B}_{s}^{0}$ mixing via top quarks. 23

2.3 Hadronic decay of a $\bar{B}^{0}$ in the spectator model. 29

2.4 More realistic representation of a hadronic decay. 29

$2.5 \quad$ Feynman diagram for $B_{s}^{0} \rightarrow J / \psi \phi$

$2.6 \quad$ Feynman diagram for $B_{d}^{0} \rightarrow J / \psi K^{* 0}$

2.7 Leading order diagrams for $b$-quark production at the Tevatron. 35

3.1 Fermilab accelerators schematic. $\quad 37$

3.2 Side view of the D $\varnothing$ Run II detector. 38

3.3 The DØ tracking system. $\quad 40$

3.4 The Silicon Microstrip Tracker.

3.5 The liquid-argon calorimeter. $\quad 45$

3.6 Level 1 and 2 trigger overview. 48

4.1 The local coordinate system of SMT ladders and wedges. 52

4.2 Illustration for equation 4.1 . 53

4.3 The global coordinate system at D $\varnothing$. 54

$\begin{array}{lll}4.4 & \text { Ghost killing. } & 57\end{array}$

4.5 Spatial resolution in $x y$ for the SMT barrels. 60

4.6 Spatial resolution in $z$ for the SMT barrels. 60

4.7 Efficiency and misidentification for 2d clusters. $\quad 61$

4.8 Efficiency and misidentification for 3d clusters. $\quad 61$ 
4.9 Spatial resolution for F-Disks. $\quad 62$

4.10 Spatial resolution for H-Disks. $\quad 62$

4.11 Timing for clustering. $\quad 64$

4.12 Pedestals for channels on a chip at the end of a ladder. 65

4.13 Influence of dead channels on pedestal fit. 66

4.14 Difference between fitted and measured pedestal divided by the error on $\begin{array}{ll}\text { the measured pedestal. } & 67\end{array}$

$\begin{array}{ll}4.15 & \text { Setup for the cosmic ray test. }\end{array}$

4.16 Variables used by the cosmic track finder. $\quad 69$

4.17 Event display for a cosmic track from online reconstruction. $\quad 70$

4.18 Event display for a cosmic track from offline reconstruction. $\quad 70$

$\begin{array}{ll}4.19 \text { Track residuals in } x \text { for cosmics. } & 71\end{array}$

$\begin{array}{ll}4.20 \text { Track residuals in } z \text { for cosmics. } & 71\end{array}$

4.21 Track residuals in $z$ for cosmics from offline reconstruction. $\quad 72$

$\begin{array}{lll}5.1 & \text { Muon reconstruction: } p_{t} \text { spectra. } & 79\end{array}$

5.2 Muon reconstruction: $p_{x}$ and $p_{z}$ resolution. $\quad 79$

5.3 Muon reconstruction: $p_{t}$ resolution as a function of $p_{t}$. 80

5.4 Muon reconstruction: Purity. $\quad 80$

5.5 Monte Carlo $J / \psi$ mass resolution. $\quad 82$

5.6 Muon reconstruction: Number of muon candidates per event. $\quad 82$

$5.7 \mathrm{~J} / \psi$ transverse momentum.

$5.8 \mathrm{~J} / \psi$ momentum resolution.

$5.9 J / \psi \chi^{2}$ distribution. $\quad 84$

$5.10 \phi$ reconstruction. $\quad 85$

$5.11 \phi$ transverse momentum.

5.12 Invariant mass of $B_{s}^{0}$ candidates (minimum cuts). $\quad 87$

5.13 Invariant mass of $B_{s}^{0}$ candidates passing all cuts. $\quad 87$

5.14 Transverse momentum for reconstructed and generated $B_{s}^{0}$. 88

$5.15 \chi^{2}$ distribution for $B_{s}^{0}$ candidates. $\quad 88$

$\begin{array}{ll}5.16 & \text { Number of minimum bias events. }\end{array}$ 
5.17 The reconstructed and MC primary vertex and the MC B-momentum. 91

5.18 Projected residual $\left(\Delta \vec{r} \cdot \vec{p}_{B}\right)$ for the primary vertex. 91

5.19 Primary vertex resolution in $x$. 92

5.20 Primary vertex resolution in $z$. 92

$\begin{array}{lll}5.21 & B_{s}^{0} \text { secondary vertex resolution. } & 93\end{array}$

5.22 Proper decay length resolution in the channel $B_{s}^{0} \rightarrow J / \psi \phi . \quad 96$

5.23 Transverse decay length resolution. $\quad 96$

$\begin{array}{ll}5.24 B_{s}^{0} \text { proper decay length distribution in the signal region. } & 99\end{array}$

$\begin{array}{lll}5.25 & B_{s}^{0} \text { proper decay length distribution in the sidebands. } & 99\end{array}$

$\begin{array}{ll}5.26 K-\pi \text { ambiguity. } & 101\end{array}$

5.27 Invariant mass of $B_{d}^{0}$ candidates (minimum cuts). 101

$5.28 K^{* 0}$ transverse momentum. 102

$5.29 B_{d}^{0}$ secondary vertex resolution. 102

$\begin{array}{llll}5.30 & B_{d}^{0} \chi^{2} \text { distribution. } & 103\end{array}$

5.31 Invariant mass of $B_{d}^{0}$ candidates after applying all cuts. 103

5.32 Proper decay length resolution in the channel $B_{d}^{0} \rightarrow J / \psi K^{* 0}$. 104

$\begin{array}{ll}5.33 B_{d}^{0} \text { proper decay length distribution in signal region. } & 107\end{array}$

$\begin{array}{ll}5.34 B_{d}^{0} \text { proper decay length distribution in sideband region. } & 107\end{array}$

$\begin{array}{ll}5.35 \text { Cross-talk. } & 109\end{array}$

5.36 Transversity angle as simulated by QQ. 112

5.37 Transversity angle as simulated by EvtGen. 112

5.38 Transversity angle distribution at generator level. 113

5.39 Transversity angle distribution in the $B_{s}^{0} \rightarrow J / \psi \phi$ signal sample. $\quad 114$

5.40 Real data: Mass distribution for $\mu$-pairs. Minimal cuts only. 115

5.41 Real data: Transverse momentum vs. invariant mass of muon pairs. 116

5.42 Real data: Pseudo-rapidity $\eta$ vs. invariant mass of $\mu$-pairs. 116

5.43 Real data: Mass distribution for $\mu$-pairs after applying all cuts. 117

$\begin{array}{lll}6.1 & \text { DNA structure. } & 119\end{array}$

6.2 The four bases of DNA. 120

$\begin{array}{lll}\text { 6.3 Traditional DNA sequencing. } & 121\end{array}$ 
6.4 Lambert-Beer's law. 123

$\begin{array}{lll}6.5 & \mu \text { DiaGene schematic. } & 125\end{array}$

$\begin{array}{lll}6.6 & \text { Vertexing algorithm. } & 127\end{array}$

$\begin{array}{ll}\text { 6.7 Velocity sorting algorithm. } & 127\end{array}$

$\begin{array}{lll}6.8 & \text { MEDUSA schematic } & 128\end{array}$

6.9 Fits to measured velocities. 132

6.10 MEDUSA: Simulation and data from measurement. $\quad 134$ 


\section{List of Tables}

2.1 Experimental results for $B$-hadron lifetimes.

3.1 Planned Tevatron parameters for Run II. 37

3.2 Silicon Microstrip Tracker detector parameters. 42

$\begin{array}{lll}3.3 & \text { Trigger rates for Run II. } & 47\end{array}$

4.1 SMT cluster resolutions. $\quad 63$

5.1 Particle masses according to the Particle Data Group. 77

$\begin{array}{lll}5.2 & \text { Summary of cuts used for } B_{s}^{0} \text { reconstruction. } & 89\end{array}$

5.3 Results from the unbinned maximum likelihood fit for the $B_{s}^{0}$ lifetime. $\quad 98$

$\begin{array}{lll}5.4 & \text { Summary of cuts used for } B_{d}^{0} \text { reconstruction. } & 105\end{array}$

5.5 Results from the unbinned maximum likelihood fit for the $B_{d}^{0}$ lifetime. $\quad 106$

5.6 Event yields for Run IIa. 110 


\section{Chapter 1}

\section{Introduction}

In this thesis I describe my contribution to the $\mathrm{D} \varnothing$ experiment at Fermilab in preparation for Run II. This work is divided into two parts: Work for the Level 3 trigger and Monte Carlo studies of $B$-mesons. The last part of the thesis concerns a completely different project: The development of a DNA sequencing chip as an example of HEP technology transfer.

\subsection{The Standard Model}

The Standard Model of particle physics used to describe three of the four fundamental forces of nature has proven to be a hugely successful theory. Even precision measurements have so far found no deviations from its predictions, with the exception of the neutrino masses. But when indications of massive neutrinos were found [1] they could be easily incorporated into the existing model.

A number of problems remain though: The Standard Model makes no provision for including gravity and it requires a large number of free parameters: three gauge couplings, nine Fermion masses (twelve when incorporating massive neutrinos), four quark mixing parameters and two parameters describing the Higgs potential. Not all parameters of the Standard Model have been measured to the same precision 
and we have yet to discover the Higgs.

I have concentrated on a particular aspect of the Standard Model: Measuring the decay $B_{s}^{0} \rightarrow J / \psi \phi$ and the related decay of $B_{d}^{0} \rightarrow J / \psi K^{* 0}$. Both decays have been measured before by other experiments, but never by $\mathrm{D} \emptyset$. Reproducing the results will provide a test for the upgraded $\mathrm{D} \emptyset$ detector and its newly developed software. Once this has been achieved the goal will be to improve the earlier measurements and check for inconsistencies with the Standard Model.

In chapter 2 the different aspects concerning the theory of $B$-mesons are presented. This is followed by a description of the experimental apparatus including an overview of the different trigger stages for the $\mathrm{D} \emptyset$ detector. Chapter 5 contains the results of my Monte Carlo studies. It concludes with first results from real data taken during the commissioning period of the detector.

\subsection{Triggering and reconstruction software}

Before an attempt to reconstruct particles in a detector can be made the raw data have to be transformed into more concrete objects: clusters, tracks or jets are typical examples.

As most of the collisions between the proton and anti-proton bunches result in events with no or little useful information (usually referred to as minimum bias events) the detector relies on triggers to filter out the interesting events. These events comprise less than $1 \%$ of all events occurring in the detector. Whether the triggers are hardware or software based, they have to be fast and reliable, as any data lost at trigger stage are not recoverable.

Chapter 4 describes my work for the Level 3 trigger. The Level 3 unpacking tool for the Silicon-Microstrip-Tracker delivers a set of two dimensional clusters, which are not always suitable for higher-level triggers. I developed the tool to combine these clusters into three-dimensional clusters that provide $x, y, z$ coordinates. Then I analysed its performance and that of the unpacking tool using Monte Carlo data. 
When the first data from a test-stand became available, I analysed these data by developing a cosmic track finder, which confirmed that the tools were working properly.

\subsection{Technology transfer}

High Energy Physics (HEP) is expensive. The accelerators and detectors used have been getting bigger and more sophisticated over time. Over the years research in particle physics has spilled over into other disciplines with the creation of the World Wide Web at CERN as one of the most famous examples. Yet few attempts have been made to systematically exploit the achievements of HEP.

The HEP group at Imperial College has actively and successfully encouraged technology transfer over a period of years. One of these projects, a biotechnology development, is presented here. Chapter 6 starts with an introduction to the $\mu$ DiaGene project, which aims to develop a fast and poison free DNA sequencing chip. When I joined the project preliminary measurements had been made. Based on these measurements and theoretical considerations I developed a simulation used to research different possible configurations and to provide Monte Carlo data for the development of the analysis software. 


\section{Chapter 2}

\section{$B$-mesons: CP-violation, mixing and lifetimes}

The aim of this chapter is to provide a brief overview of the theoretical aspects of the main $b$-physics topics at $\mathrm{D} \varnothing$. A summary of the experimental studies conducted so far can be found in section 7.1.

This chapter starts with a brief introduction to the concept of symmetries in the Standard Model. Then the formalism of the CKM matrix is presented and a description of mixing in the neutral $B$-meson systems is given. This is followed by a discussion of the different types of $C P$-violation within the Standard Model. $B$-mesons at the Tevatron are produced with a large boost. This makes $\mathrm{D} \varnothing$ an ideal environment for studying their lifetimes. The relevant theory is presented in section 2.6. The following two sections give the details of the decays studied in this thesis. The chapter concludes with a brief discourse on $b$-quark production, as the $b \bar{b}$ crosssection is later used to calculate the expected event rates in the signal channels.

\subsection{Symmetries in the Standard Model}

The Standard Model describes the strong, weak and electromagnetic interactions in terms of renormalizable gauge theories [2]. Gauge theories are based on invariance 
under a set of local (i.e. space-time dependent) transformations. In order for a gauge theory to be physically meaningful it has to be renormalizable. This means it must be possible to absorb the divergences that appear as higher-order corrections into the redefinition of the theory's free parameters.

Symmetries, whether conserved or broken, play a major role in all quantum field theories. The Standard Model uses a $S U(3) \times S U(2) \times U(1)$ gauge symmetry to describe the strong $(S U(3))$ and electroweak $(S U(2) \times(U(1))$ interactions. The spontaneous symmetry breaking of $S U(2) \times U(1)$ (Higgs mechanism) generates the particle masses while preserving the renormalizability of the theory.

While gauge invariance and renormalizability already severely restrict the form of the Standard Model Lagrangian, there are three further symmetries to be taken into account:

\section{Parity}

Parity symmetry $P$ consists of the invariance of physics under a discrete transformation which changes the sign of the space coordinates $x, y, z$. A right-handed coordinate system becomes left-handed under this transformation. Parity is conserved in strong and electromagnetic interactions. It is violated in weak interactions, most prominently in the neutrinos: In the limit of vanishing neutrino masses all neutrinos are left-handed ${ }^{1}$ and all $^{2}$ anti-neutrinos are right-handed. For this reason the weak isospin group is usually referred to as $S U(2)_{L}$ as only the left-handed fermion fields participate in weak interactions ${ }^{3}$. It is $U(1)_{Y}$ associated with the hypercharge $Y$ ( $Q=T_{3}+Y / 2$ with charge $Q$ and $T_{3}$ the third component of the weak isospin) which couples to both helicity states.

\footnotetext{
${ }^{1}$ Left-handed neutrino: The projection of its spin is antiparallel to the direction of motion.

${ }^{2} \mathrm{~A}$ non-zero mass means it is possible to perform a Lorentz transformation and change a lefthanded neutrino into a right-handed neutrino and vice versa.

${ }^{3}$ The weak isospin for right-handed leptons and quarks is $0\left(T=T_{3}=0\right)$.
} 


\section{Charge conjugation}

Charge conjugation $C$ converts a particle into its anti-particle. It changes the sign of all internal quantum numbers such as charge, baryon number and strangeness while leaving mass, energy, momentum and spin unchanged. Only particles that are their own anti-particles can be eigenstates of $C$. Like parity, $C$ is conserved for strong and electromagnetic interactions, but violated in weak decays:

$$
\begin{array}{lll}
\Gamma\left(\pi^{+} \rightarrow \mu^{+} \nu_{L}\right)=99.98 \% \neq \Gamma\left(\pi^{+} \rightarrow \mu^{+} \nu_{R}\right)=0 & \mathrm{P} \text { violation } \\
\Gamma\left(\pi^{+} \rightarrow \mu^{+} \nu_{L}\right)=99.98 \% \neq \Gamma\left(\pi^{-} \rightarrow \mu^{-} \bar{\nu}_{L}\right)=0 & \mathrm{C} \text { violation }
\end{array}
$$

but

$$
\Gamma\left(\pi^{+} \rightarrow \mu^{+} \nu_{L}\right)=\Gamma\left(\pi^{-} \rightarrow \mu^{-} \bar{\nu}_{R}\right)
$$

\section{Time reversal and the CPT theorem}

Time reversal $T$ reverses the sign of the time coordinate, i.e. it swaps the initial and final state. The invariance under the product of all three symmetries is thought to always hold for any Lorentz invariant local quantum field theory. This is known as the $C P T$ theorem. A consequence of $C P T$ invariance is that the masses of a particle and its anti-particle should be equal. This has been verified for example in the kaon system and it was found that $\frac{\left|m_{K^{0}}-m_{\bar{K}^{0}}\right|}{m_{\text {average }}}<10^{-18}[3]$.

Parity violation in weak interactions was discovered in 1957 [4]. For a long time it was thought that $C P$ was conserved until in 1964 Christenson, Cronin and Turlay found evidence for $C P$-violation in the neutral kaon system [5].

\subsection{The discovery of CP-violation}

Neutral kaons are observed as $K_{L}^{0}$ with a lifetime $\tau=(5.17 \pm 0.04) \times 10^{-8} \mathrm{~s}$ and as $K_{S}^{0}$ with a lifetime of $\tau=(0.8935 \pm 0.0008) \times 10^{-10} \mathrm{~s}[3]$. These kaons are a mixture 
of the flavour eigenstates $K^{0}$ and $\bar{K}^{0}$ :

$$
\begin{aligned}
& \left|K_{L}^{0}\right\rangle=p\left|K^{0}\right\rangle+q\left|\bar{K}^{0}\right\rangle \\
& \left|K_{S}^{0}\right\rangle=p\left|K^{0}\right\rangle-q\left|\bar{K}^{0}\right\rangle
\end{aligned}
$$

If $C P$ is conserved then $q=p$ and $K_{S}^{0}$ and $K_{L}^{0}$ would be eigenstates of $C P$, with eigenvalues +1 and -1 respectively.

The original experiment looked at the decay of neutral $K$ mesons into pions. The short-lived $C P$-eigenstate should always decay into two pions, the long-lived eigenstate into three. But what Christenson et al. found was that a tiny fraction of the $K_{L}^{0}$ decayed into two pions and therefore $C P$ was not conserved.

The experimental quantities measured are the ratios of the decay amplitudes

$$
\begin{gathered}
\eta_{+-}=\frac{A\left(K_{L} \rightarrow \pi^{+} \pi^{-}\right)}{A\left(K_{S} \rightarrow \pi^{+} \pi^{-}\right)}=\left|\eta_{+-}\right| e^{i \phi_{+-}} \\
\eta_{00}=\frac{A\left(K_{L} \rightarrow \pi^{0} \pi^{0}\right)}{A\left(K_{S} \rightarrow \pi^{0} \pi^{0}\right)}=\left|\eta_{00}\right| e^{i \phi_{00}}
\end{gathered}
$$

and their current values are [3]

$$
\begin{aligned}
& \left|\eta_{+-}\right|=(2.276 \pm 0.017) \times 10^{-3} \quad \phi_{+-}=(43.3 \pm 0.5)^{\circ} \\
& \left|\eta_{00}\right|=(2.262 \pm 0.017) \times 10^{-3} \quad \phi_{00}=(43.2 \pm 1.0)^{\circ}
\end{aligned}
$$

\subsection{The CKM matrix}

The weak interaction couples to the quark doublets $\left(\begin{array}{c}u \\ d^{\prime}\end{array}\right),\left(\begin{array}{l}c \\ s^{\prime}\end{array}\right),\left(\begin{array}{l}t \\ b^{\prime}\end{array}\right)$, where $d^{\prime}, s^{\prime}, b^{\prime}$ are linear combinations of the physical quarks $d, s$ and $b$, which are produced through rotation in flavour space.

$$
\left(\begin{array}{c}
\left|d^{\prime}\right\rangle \\
\left|s^{\prime}\right\rangle \\
\left|b^{\prime}\right\rangle
\end{array}\right)=\mathbf{V}_{\mathrm{CKM}} \cdot\left(\begin{array}{c}
|d\rangle \\
|s\rangle \\
|b\rangle
\end{array}\right)=\left(\begin{array}{lll}
V_{u d} & V_{u s} & V_{u b} \\
V_{c d} & V_{c s} & V_{c b} \\
V_{t d} & V_{t s} & V_{t b}
\end{array}\right)\left(\begin{array}{c}
|d\rangle \\
|s\rangle \\
|b\rangle
\end{array}\right)
$$


$\mathbf{V}_{\text {CKM }}$ is known as the Cabibbo-Kobayashi-Maskawa matrix. Its elements $V_{i j}$ are complex numbers, measuring the coupling between quarks of different flavours. It is unitary $\left(\boldsymbol{V} \boldsymbol{V}^{+}=1\right)$ by construction. Of its nine parameters a global phase, two relative phases between the $u, c, t$-quarks and two relative phases between the $d$, $s, b$-quarks are not observable. This leaves three angles and a complex phase $\delta$, related to the coupling of the $W$ to the quarks. This phase is used to incorporate $C P$-violation into the Standard Model. As it is possible to omit this phase in a theory with two quark generations, Kobayashi and Maskawa originally proposed the existence of the third generation in order to provide a mechanism for $C P$-violation [6]. In this parameterization $\mathbf{V}_{\text {CKM }}$ becomes

$$
\mathbf{V}_{\mathrm{CKM}}=\left(\begin{array}{ccc}
c_{12} c_{13} & s_{12} c_{13} & s_{13} e^{-i \delta} \\
-s_{12} c_{23}-c_{12} s_{13} s_{23} e^{i \delta} & c_{12} c_{23}-s_{12} s_{13} s_{23} e^{i \delta} & c_{13} s_{23} \\
s_{12} s_{23}-c_{12} s_{13} c_{23} e^{i \delta} & -c_{12} s_{23}-s_{12} s_{13} c_{23} e^{i \delta} & c_{13} c_{23}
\end{array}\right)
$$

where $c_{i j}=\cos \theta_{i j}, s_{i j}=\sin \theta_{i j}$. The angles $\theta_{i j}$ vary between 0 and $\pi / 2$, the phase $\delta$ between 0 and $2 \pi$.

The Wolfenstein [7] parameterization in $A, \lambda, \rho, \eta$ is an expansion in $\lambda=\left|V_{u s}\right|=0.22$ with four independent parameters. The parameter $\eta$ takes the role of the $\mathrm{CP}$ violating phase $\delta$.

$$
\mathbf{V}_{\mathrm{CKM}} \approx\left(\begin{array}{ccc}
1-\frac{\lambda^{2}}{2} & \lambda & A \lambda^{3}(\rho-i \eta) \\
-\lambda & 1-\frac{\lambda^{2}}{2} & A \lambda^{2} \\
A \lambda^{3}(1-\rho-i \eta) & -A \lambda^{2} & 1
\end{array}\right)
$$

Unitarity imposes a number of restrictions on the matrix elements. The most useful ones are

$$
V_{u d} V_{u b}^{*}+V_{c d} V_{c b}^{*}+V_{t d} V_{t b}^{*}=0
$$




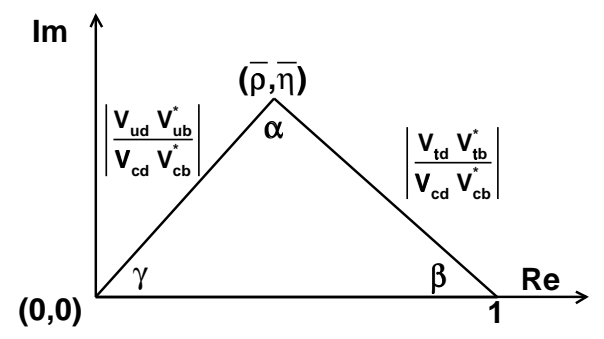

(a) Unitarity triangle for equation 2.7

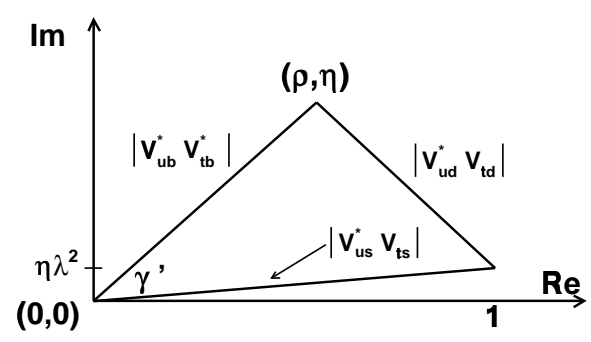

(b) Unitarity triangle for equation 2.8

Figure 2.1: Two unitarity triangles of the CKM matrix. The parameters $\bar{\rho}$ and $\bar{\eta}$ are defined as $\bar{\rho} \equiv\left(1-\lambda^{2} / 2\right) \rho$ and $\bar{\eta} \equiv\left(1-\lambda^{2} / 2\right) \eta$.

and

$$
V_{u d}^{*} V_{t d}+V_{u s}^{*} V_{t s}+V_{u b}^{*} V_{t b}=0
$$

These conditions can be represented as triangles in the complex plane (fig. 2.1). In the Wolfenstein parameterization up to $\lambda^{3}$ both triangles are identical and are usually referred to as the unitarity triangle. The angles of the unitary triangle are then defined as:

$$
\alpha \equiv \arg \left[-\frac{V_{t d} V_{t b}^{*}}{V_{u d} V_{u b}^{*}}\right], \quad \beta \equiv \arg \left[-\frac{V_{c d} V_{c b}^{*}}{V_{t d} V_{t b}^{*}}\right], \quad \gamma \equiv \arg \left[-\frac{V_{u d} V_{u b}^{*}}{V_{c d} V_{c b}^{*}}\right] .
$$

Like most perturbative expansions, the higher order terms are not unique and different papers will quote different formulae [7], [8]. A common parameterisation of the higher orders of the CKM matrix is defined by imposing the relations

$$
s_{12}=\lambda \quad s_{23}=A \lambda^{2} \quad s_{13} e^{-i \delta}=A \lambda^{3}(\rho-i \eta)
$$


to all orders of $\lambda$. This leads to the following extension:

$$
\mathbf{V}_{\mathrm{CKM}} \approx \mathbf{V}_{\mathrm{CKM}}\left(\lambda^{3}\right)+\left(\begin{array}{ccc}
-\frac{\lambda^{4}}{8} & 0 & 0 \\
-A^{2} \lambda^{5}\left(\rho+i \eta-\frac{1}{2}\right) & -\left(\frac{1}{8}+\frac{A}{2}\right) \lambda^{4} & 0 \\
\frac{1}{2} A \lambda^{5}(\rho+i \eta) & -A \lambda^{4}\left(\rho+i \eta-\frac{1}{2}\right) & -\frac{A^{2}}{2} \lambda^{4}
\end{array}\right)
$$

With this extension the $C P$-violating phase $\eta$ appears also in the $V_{t s}$ and $V_{c d}$ matrix elements and the two triangles become distinct from each other (see fig. 2.1). This effect is beyond the reach of the Tevatron experiments, but will be relevant for LHC measurements $[9]$.

\subsection{Mixing in the neutral B-meson system}

Particle-antiparticle mixing, as in the neutral kaon system, and oscillations between the flavour eigenstates also exist in the $B_{d}^{0}$ and $B_{s}^{0}$ meson systems. Such a system is produced in one of the two possible states of well-defined flavour: $\left|B_{s}^{0}\right\rangle(\sim \bar{b} s)$ or $\left|\bar{B}_{s}^{0}\right\rangle(\sim b \bar{s})$. This initial state evolves into a time-dependent superposition of the two flavour states according to

$$
i \frac{\partial}{\partial t}\left(\begin{array}{c}
\left|B_{q}^{0}(t)\right\rangle \\
\left|\bar{B}_{q}^{0}(t)\right\rangle
\end{array}\right)=\left(\mathbf{M}-i \frac{\Gamma}{2}\right)\left(\begin{array}{l}
\left|B_{q}^{0}(t)\right\rangle \\
\left|\bar{B}_{q}^{0}(t)\right\rangle
\end{array}\right)
$$

where $\boldsymbol{\Gamma}=\boldsymbol{\Gamma}^{+}$and $\mathbf{M}=\mathbf{M}^{+}$are the decay and mass matrices. The mixing is due to the off-diagonal elements $M_{12}=M_{21}^{*}$ and $\Gamma_{12}=\Gamma_{21}^{*}$.

The probability density to observe an initial $B_{q=d, s}^{0}$ meson decaying as a $B_{q}^{0}$ or $\bar{B}_{q}^{0}$ meson at a time $t$ after its creation is given by [9]

$$
P(t)=\frac{\Gamma^{2}-\left(\frac{\Delta \Gamma}{2}\right)^{2}}{2 \Gamma} e^{-\Gamma t}\left[\cosh \frac{\Delta \Gamma t}{2}+\mu \cos (\Delta m t)\right]
$$

with $\mu=-1$ for $B_{q}^{0} \rightarrow \bar{B}_{q}^{0}$ and $\mu=+1$ for $B_{q}^{0} \rightarrow B_{q}^{0}$. The decay width difference $\Delta \Gamma$ is defined as $\Gamma_{\text {Heavy }}-\Gamma_{L \text { ight }}$ and $\Gamma=\frac{\Gamma_{H}+\Gamma_{L}}{2}$. When measuring the time-dependent 

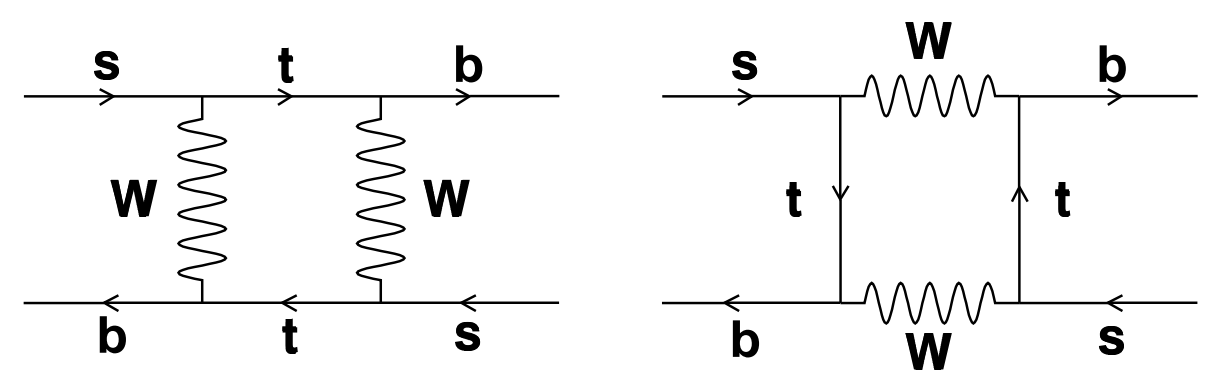

Figure 2.2: Box diagrams for $B_{s}^{0} \leftrightarrow \bar{B}_{s}^{0}$ mixing via top quarks.

asymmetry

$$
A(t)=\frac{P_{B_{q}^{0} \rightarrow B_{q}^{0}}(t)-P_{B_{q}^{0} \rightarrow \bar{B}_{q}^{0}}(t)}{P_{B_{q}^{0} \rightarrow B_{q}^{0}}(t)+P_{B_{q}^{0} \rightarrow \bar{B}_{q}^{0}}(t)}=\frac{\cos (\Delta m t)}{\cosh \frac{\Delta \Gamma t}{2}}
$$

the mass difference $\Delta m$ is proportional to the oscillation frequency, while a non-zero width difference will result in a time-dependent damping of the oscillations.

The matrix element $M_{12}$ can be calculated from the box diagrams (see fig. 2.2) which are dominated by the exchange of virtual top quarks. This leads to

$$
M_{12}=\frac{G_{F}^{2} m_{W}^{2} \eta_{B} B_{B_{q}} f_{B_{q}}^{2}}{12 \pi^{2}} S_{0}\left(\frac{m_{t}^{2}}{m_{W}^{2}}\right)\left|V_{t q}^{*} V_{t b}\right|^{2}
$$

where $G_{F}$ is the Fermi constant, $m_{W}$ the $W$ mass, $V_{t q=d, s}$ and $V_{t b}$ the relevant CKM matrix elements. The function $S_{0}$ can be approximated by $0.784\left(\frac{m_{t}^{2}}{m_{W}^{2}}\right)^{0.76}$ and the QCD correction $\eta_{B}$ is of order unity [3]. The decay constant $f_{B}$ and the bag-parameter $B_{B_{q}}$ have been determined using lattice calculations. Their current values are [9]

$$
f_{B}=(200 \pm 40) \mathrm{MeV}, \quad f_{B_{s}}=(230 \pm 40) \mathrm{MeV}, \quad \frac{f_{B_{s}}}{f_{B}}=1.15 \pm 0.07
$$

and

$$
B_{B_{q}}\left(m_{b}\right)=0.91 \pm 0.06, \quad \frac{B_{B_{s}}}{B_{B}}=1.00(3)
$$


Assuming $C P$-violation in mixing is negligible [10] the mass difference between the heavy and light eigenstate is related to $M_{12}$ by

$$
\Delta m=2\left|M_{12}\right|
$$

and

$$
\frac{\Delta m_{B_{s}}}{\Delta m_{B_{d}}} \approx \frac{f_{B_{s}}^{2}}{f_{B_{d}}^{2}} \frac{B_{B_{s}}}{B_{B_{d}}}\left|\frac{V_{t s}}{V_{t d}}\right|^{2}
$$

While the mass difference $\Delta m_{B_{d}^{0}}$ has been measured to be $(0.472 \pm 0.017) \times 10^{12} \hbar s^{-1}$ [3], the rapid oscillations in the $B_{s}^{0}$ system have not yet been resolved. Measurements give $x_{s}=\frac{\Delta m_{B_{s}}}{\Gamma}>15.7$ at $95 \%$ confidence level [3].

The matrix element $\Gamma_{12}$ describes real transitions due to decay modes common to the particle and anti-particle (e.g. $B_{d}^{0}\left(\bar{B}_{d}^{0}\right) \rightarrow \pi^{+} \pi^{-}$). These decays involve $b \rightarrow c \bar{c} q$ transitions, which are Cabbibo suppressed for $q=d$, but favoured for $q=s$. Therefore the width difference in the $B_{d}^{0}$ system is extremely small and usually assumed to be zero, but the width difference in the $B_{s}^{0}$ system might be sizeable. Theoretical predictions [11] yield

$$
\frac{\Delta \Gamma_{s}}{\Gamma_{s}}=0.12 \pm 0.06 \%
$$

A measurement [12] by the ALEPH experiment in the $B_{s}^{0}\left(\overline{B_{s}^{0}}\right) \rightarrow D_{s}^{(*)+} D_{s}^{(*)-}$ channel using

$$
2 \operatorname{BR}\left(B_{s}^{0}\left(\overline{B_{s}^{0}}\right) \rightarrow D_{s}^{(*)+} D_{s}^{(*)-}\right) \simeq \frac{\Delta \Gamma}{\Gamma_{L}}
$$

gives

$$
\frac{\Delta \Gamma}{\Gamma_{L}}=0.26_{-0.15}^{+0.30}
$$

which is consistent with the theoretical predictions. 


\subsection{Types of CP-violation}

There are three basic types of $C P$-violation:

$\boldsymbol{C P}$-violation in the mixing of neutral mesons as in the kaon example above. This type of $C P$-violation is also known as indirect $C P$-violation.

$\boldsymbol{C P}$-violation in the decay of neutral and charged mesons, also referred to as direct $C P$-violation. Here the transition rate $T$ from an initial state $i$ to a final state $f$ of a decay and its $C P$ conjugate process are different: $|\langle f|T| i\rangle|^{2} \neq|\langle\bar{f}|T| \bar{i}\rangle|^{2}$.

This type of $C P$-violation has been measured by the KTEV [13] and NA48 [14] experiments using

$$
R=\frac{\Gamma\left(K_{L} \rightarrow \pi^{0} \pi^{0}\right) / \Gamma\left(K_{S} \rightarrow \pi^{0} \pi^{0}\right)}{\Gamma\left(K_{L} \rightarrow \pi^{+} \pi^{-}\right) / \Gamma\left(K_{S} \rightarrow \pi^{+} \pi^{-}\right)} \simeq 1-6 \operatorname{Re}\left(\epsilon^{\prime} / \epsilon\right)
$$

where $\epsilon^{\prime}$ refers to direct and $\epsilon$ to indirect $C P$-violation. A non-zero value of $R e\left(\epsilon^{\prime} / \epsilon\right)$ indicates direct $C P$-violation. The current experimental value is [3]

$$
\operatorname{Re}\left(\epsilon^{\prime} / \epsilon\right)=(2.1 \pm 0.5) \times 10^{-3}
$$

$\boldsymbol{C P}$-violation in the interference between decays with and without mixing. This type of decay can for example be observed in the neutral $B_{d}^{0}$ system [10], [15]. The light and heavy mass eigenstates of the $B_{d}^{0}$ can be described by

$$
\begin{aligned}
& \left|B_{L}\right\rangle=p\left|B^{0}\right\rangle+q\left|\bar{B}^{0}\right\rangle \\
& \left|B_{H}\right\rangle=p\left|B^{0}\right\rangle-q\left|\bar{B}^{0}\right\rangle
\end{aligned}
$$

where the complex coefficients $p$ and $q$ are normalized so that

$$
|p|^{2}+|q|^{2}=1
$$


The decay amplitudes $A_{f}$ are defined as

$$
\begin{array}{ll}
A_{f}=\left\langle f|T| B^{0}\right\rangle, & A_{\bar{f}}=\left\langle\bar{f}|T| B^{0}\right\rangle \\
\bar{A}_{f}=\left\langle f|T| \bar{B}^{0}\right\rangle, & \bar{A}_{\bar{f}}=\left\langle\bar{f}|T| \bar{B}^{0}\right\rangle
\end{array}
$$

and the parameter $\lambda$ by

$$
\lambda_{f_{C P}} \equiv \frac{q}{p} \frac{\bar{A}_{f}}{A_{f}}=\eta_{f_{C P}} \frac{q}{p} \frac{\bar{A}_{\bar{f}}}{A_{f}}
$$

where $\eta_{f_{C P}}= \pm 1$ is the $C P$-eigenvalue of the state $f_{C P} \cdot C P$-violation leads to $\lambda \neq \pm 1$. Note that $C P$-violation is possible with $|\lambda|=1$ as long as $\operatorname{Im}(\lambda) \neq 0$. The time-dependent asymmetry $a_{f_{C P}}$ in this type of $C P$-violation can be observed by comparing decays into final $C P$-eigenstates

$$
a_{f_{C P}}=\frac{\Gamma\left(B^{0}(t) \rightarrow f_{c p}\right)-\Gamma\left(\bar{B}^{0}(t) \rightarrow f_{c p}\right)}{\Gamma\left(B^{0}(t) \rightarrow f_{c p}\right)+\Gamma\left(\bar{B}^{0}(t) \rightarrow f_{c p}\right)}
$$

The asymmetry $a_{f_{C P}}$ is related to $\lambda$ by [15]

$$
a_{f_{C P}}=\frac{\left(1-|\lambda|^{2}\right) \cos \left(\Delta m_{b} t\right)-2 \operatorname{Im}(\lambda) \sin \left(\Delta m_{B} t\right)}{1+|\lambda|^{2}}
$$

For $B_{d}^{0}$ decays that are dominated by a single $C P$-violating phase (i.e. the effect of $C P$-violation in decay is negligible), $a_{f_{C P}}$ simplifies to

$$
a_{f_{C P}}=\operatorname{Im}(\lambda) \sin \Delta m_{B} t
$$

This is the case in the 'golden' decay $B_{d}^{0} \rightarrow J / \psi\left(\rightarrow l^{+} l^{-}\right) K_{s}^{0}\left(\rightarrow \pi^{+} \pi^{-}\right)$where the final state is common to both the $B^{0}$ and the $\bar{B}^{0}$. At tree-level $\lambda$ can be written as:

$$
\lambda_{B_{d}^{0} \rightarrow J / \psi K_{s}}=-\left(\frac{V_{t b}^{*} V_{t d}}{V_{t b} V_{t d}^{*}}\right)\left(\frac{V_{c b} V_{c s}^{*}}{V_{c b}^{*} V_{c s}}\right)\left(\frac{V_{c s}^{*} V_{c b}}{V_{c s} V_{c b}^{*}}\right)
$$

The first term arises from $B^{0}$ mixing, the second term from $K^{0}$ mixing and the third term from the $B^{0}$ decay. $K^{0}$ mixing is essential as otherwise at tree level the 
$B^{0}(\sim \bar{b} d)$ will decay to a $K^{0}(\sim \bar{s} d)$ and similarly the $\bar{B}^{0}$ to a $\bar{K}^{0}$, which are not $C P$-eigenstates. The effect caused by the fact that $K_{S}$ and $K_{L}$ are not completely $C P$-eigenstates is negligible. Combining equations 2.9 and 2.32 gives

$$
\arg \lambda_{B_{d}^{0} \rightarrow J / \psi K_{s}}=2 \beta
$$

and

$$
a_{B_{d}^{0} \rightarrow J / \psi K_{s}}=\sin 2 \beta \sin \Delta m_{B} t
$$

Recent results for $\sin 2 \beta$ from BABAR [16] using a number of decays including $B_{d}^{0} \rightarrow J / \psi K_{S}^{0}$ give

$$
\sin 2 \beta=0.75 \pm 0.09 \text { (stat) } \pm 0.04 \text { (syst) }
$$

and $|\lambda|=0.93 \pm 0.06$ (stat) \pm 0.02 (syst) which is consistent with the Standard Model expectation of no direct $C P$-violation in this type of decay.

In a similar analysis the BELLE collaboration finds [17]

$$
\sin 2 \beta=0.82 \pm 0.12 \text { (stat) } \pm 0.05 \text { (syst) }
$$

\subsection{Lifetimes}

The simplest way to describe the decay of a $B$-meson is the pure spectator quark model, where the $b$-quark decays to a $c$-quark with only negligible interference from the other quark in the $B$-meson (see fig. 2.3). The $b$-quark couples predominantly to the $c$-quark and its lifetime depends essentially on the matrix element $V_{c b}$. This fact was used in early lifetime measurements of $B$-mesons [18], [19] which provided the first measurement of the magnitude of $V_{c b}$.

According to the spectator model the lifetimes of all $B$-mesons would be equal. Measuring the lifetimes probes to what extent this model is valid and to what degree 
other diagrams and final state interactions (see for example fig. 2.4) contribute to the decays.

The current experimental results are:

\begin{tabular}{|cc|}
\hline Particle & Lifetime in ps \\
\hline$B_{d}^{0}$ & $1.548 \pm 0.032$ \\
$B^{+}$ & $1.653 \pm 0.028$ \\
$B_{s}^{0}$ & $1.493 \pm 0.062$ \\
$B_{c}$ & $0.46 \pm 0.03$ \\
\hline
\end{tabular}

Table 2.1: Experimental results for $B$-hadron lifetimes [3]. The lifetime of the $B_{c}$ is significantly shorter as both quarks can decay weakly.

\section{Heavy Quark Effective Theory}

A very successful theory to describe the decay of mesons containing a heavy and a light quark is the Heavy Quark Effective Theory (HQET) [20], where a heavy quark is defined by $m_{Q} \gg \Lambda_{Q C D}$, with $\Lambda_{Q C D} \sim 0.2 \mathrm{GeV}$. In the HQET the Lagrangian is expressed in an expansion in powers of $1 / m_{Q}$, usually referred to as the Operator Product Expansion (OPE). HQET works best for bound states containing a $b$-quark, and is in most cases also valid for mesons containing $c$-quarks, even though the lower mass of the $c$-quark $(\sim 1.2 \mathrm{GeV})$ can require further corrections. It does not apply to top quarks though, as they decay before forming bound hadronic states.

In the $1 / m_{Q}$ expansion, the leading term describes the decay of a free quark. Differences in the lifetimes of $B$-mesons first appear in the $1 / m_{Q}^{3}$ terms and for the $\Lambda_{b}$ at $1 / m_{Q}^{2}$ level [21].

The lifetime ratios of $B$-mesons and the $\Lambda_{b}$ have been calculated using the OPE to be

$$
\frac{\tau_{B^{+}}}{\tau_{B^{0}}}=1 \pm\left(\frac{f_{B}}{200 M e V}\right)^{2}, \frac{\tau_{B_{s}}}{\tau_{B^{0}}}=1 \pm 0.01, \quad \frac{\Lambda_{b}}{\tau_{B^{0}}}=0.9
$$


with $f_{B}$ as in equation 2.16. The experimental values are

$$
\frac{\tau_{B^{+}}}{\tau_{B^{0}}}=1.062 \pm 0.029, \quad \frac{\tau_{B_{s}}}{\tau_{B^{0}}}=0.964 \pm 0.045, \quad \frac{\Lambda_{b}}{\tau_{B^{0}}}=0.780 \pm 0.037
$$

which are, except for the $\Lambda_{b}$, in good agreement with the theory.

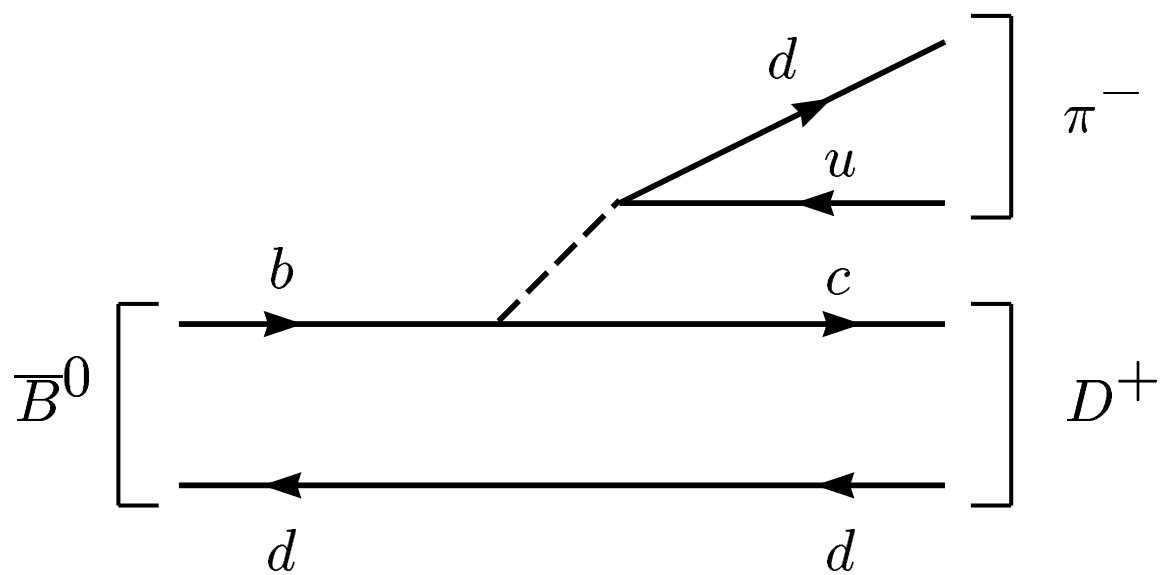

Figure 2.3: Hadronic decay of a $\bar{B}^{0}$ in the spectator model.

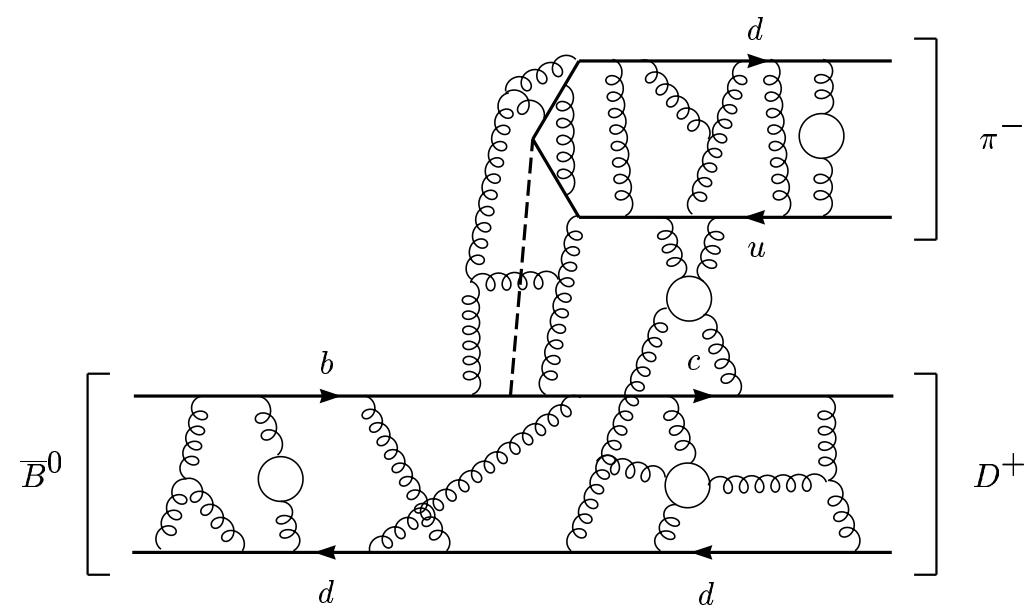

Figure 2.4: More realistic representation of a hadronic decay (diagram taken from [20]). 


\subsection{The decay $B_{s}^{0} \rightarrow J / \psi\left(\rightarrow \mu^{+} \mu^{-}\right) \phi\left(\rightarrow K^{+} K^{-}\right)$}

When the $B_{s}^{0}$ decays into a $J / \psi$ and a $\phi$, they both effectively ${ }^{4}$ decay instantaneously. In the decay $J / \psi \rightarrow \mu^{+} \mu^{-}$and $\phi \rightarrow K^{+} K^{-}$this results in a four track vertex which makes this decay an ideal candidate for measuring the $B_{s}^{0}$ lifetime. A Feynman diagram of the decay is shown in fig. 2.5.

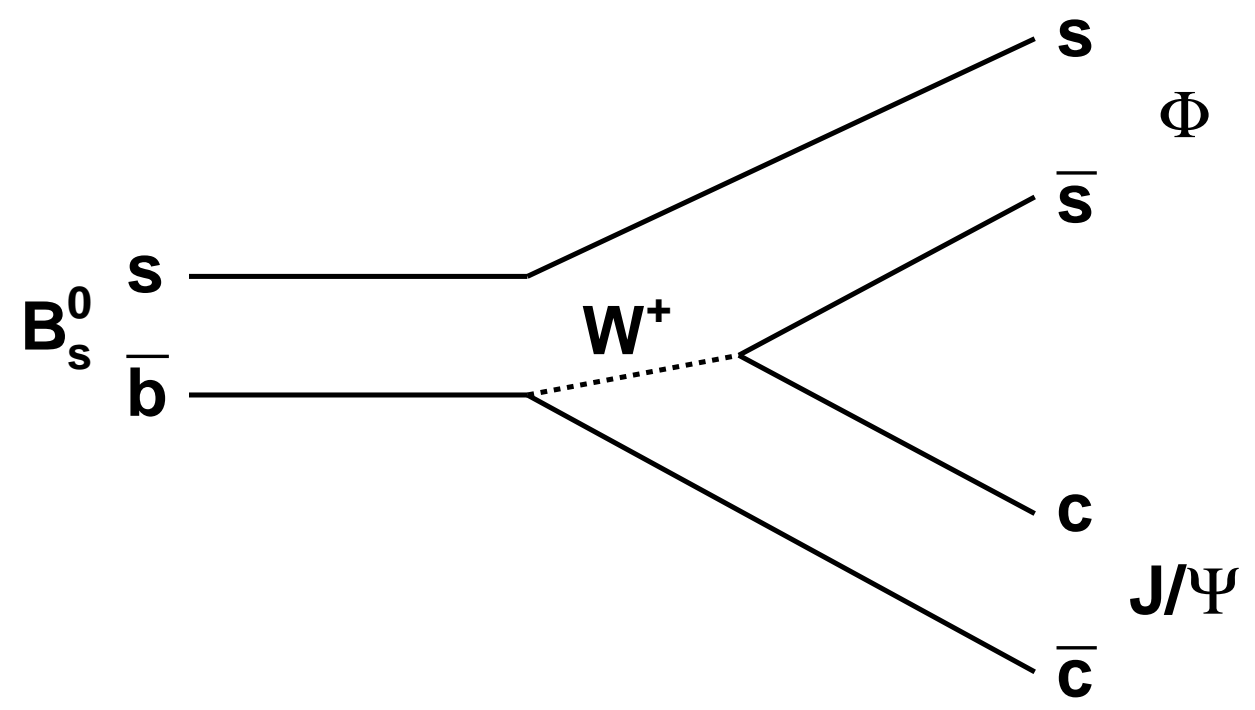

Figure 2.5: Tree level diagram for the decay $B_{s}^{0} \rightarrow J / \psi \phi$.

The decay $B_{s}^{0} \rightarrow J / \psi \phi$ has another interesting feature:

The $B_{s}^{0}$ is a spin 0 particle decaying to two vector mesons $(J / \psi, \phi)$. This implies a relative angular momentum of $\mathrm{L}=0,1,2$ between the vector mesons and the final state will be a mixture between $\mathrm{CP}$ even and odd states with $\mathrm{L}=0,2$ being $\mathrm{CP}$ even and $\mathrm{L}=1 \mathrm{CP}$ odd. The corresponding angular distributions were originally described in terms of helicity [22] which is defined as the sign of the projection of a particle's spin $\vec{s}$ along the direction of its momentum $\vec{p}$ :

$$
h=\frac{\vec{s} \cdot \vec{p}}{|\vec{s}||\vec{p}|}
$$

\footnotetext{
${ }^{4}$ The width of the $J / \psi$ is $(87 \pm 5) \mathrm{keV}$ and that of the $\phi(4.458 \pm 0.032) \mathrm{MeV}$.
} 
To separate the $C P$ even and odd contributions to the final state it is more convenient to use the so-called transversity basis [23] which describes the decay in terms of polarisations analogous to light polarisations. The three amplitudes $A_{0}, A_{\|}$and $A_{\perp}$ refer to longitudinal, parallel and perpendicular polarised states. They are related [15] to the three helicity amplitudes $H_{0}(0,0), H_{+}(1,1), H_{-}(-1,-1)$ through

$$
A_{0}=H_{0}, \quad A_{\|}=\frac{1}{\sqrt{2}}\left(H_{+}+H_{-}\right), \quad A_{\perp}=\frac{1}{\sqrt{2}}\left(H_{+}-H_{-}\right)
$$

and are normalized so that

$$
d \Gamma\left(B_{s}^{0} \rightarrow J / \psi \phi\right) / d t=\left|A_{0}\right|^{2}+\left|A_{\|}\right|^{2}+\left|A_{\perp}\right|^{2}
$$

$A_{\perp}$ refers to $\mathrm{CP}$ odd states, while $A_{\|}$and $A_{0}$ measure $\mathrm{CP}$ even states.

There are three angles which can be used to extract these amplitudes [23]. The first two are defined in a right-handed Cartesian coordinate system in the $J / \psi$ rest-frame. The $x$-axis in this frame is the direction of the $\phi$, the $y$-axis lies in the plane formed by the $K^{+}$and the $K^{-}$with $\vec{p}\left(K^{+}\right)>0$ and the $z$ axis is the normal to this plane. The angles are then

$\theta_{t}$ : The angle between the $\mu^{+}$and the $z$-axis.

$\varphi$ : The angle between the projection of the $\mu^{+}$on the $K^{+} K^{-}$plane and the $x$-axis. The third angle $\psi$ is defined in the $\phi$ rest-frame as the angle between the $K^{+}$and the negative direction of the $J / \psi$ in this frame.

The full angular distribution is

$$
\begin{array}{r}
\frac{d^{4} \Gamma\left[B_{s} \rightarrow\left(l^{+} l^{-}\right)_{J / \psi}\left(K^{+} K^{-}\right)_{\phi}\right]}{d \cos \theta_{t} d \varphi d \cos \psi}=\frac{9}{32 \pi}\left[2\left|A_{0}\right|^{2} \cos ^{2}(\psi)\left(1-\sin ^{2} \theta_{t} \cos ^{2} \varphi\right)\right. \\
+\sin ^{2} \psi\left\{\left|A_{\|}\right|^{2}\left(1-\sin ^{2} \theta_{t} \sin ^{2} \varphi\right)+\left|A_{\perp}\right| \sin ^{2} \theta_{t}-\operatorname{Im}\left(A_{\|}^{*} A_{\perp}\right) \sin 2 \theta_{t} \sin \varphi\right\} \\
\left.+\frac{1}{\sqrt{2}} \sin 2 \psi\left\{\operatorname{Re}\left(A_{0}^{*} A_{\|}\right) \sin ^{2} \theta_{t} \sin 2 \varphi+\operatorname{Im}\left(A_{0}^{*} A_{\perp}\right) \sin 2 \theta_{t} \cos \varphi\right\}\right]
\end{array}
$$


Using this method $\left|A_{\perp}\right|^{2}$ and $\left|A_{0}\right|$ have been measured [24] to be

$$
\begin{aligned}
\left|A_{\perp}\right|^{2} & =0.23 \pm 0.19 \text { (stat) } \pm 0.04 \text { (syst) } \\
\left|A_{0}\right|^{2} & =\frac{\Gamma_{\text {longitudinal }}}{\Gamma}=0.61 \pm 0.14 \text { (stat) } \pm 0.02 \text { (syst) }
\end{aligned}
$$

Depending on the analysis it is often sufficient only to measure the parameter $\cos \left(\theta_{t}\right)$ which is referred to as the transversity variable. The probability density function for CP even decays is then $\frac{3}{8}\left(1+\cos ^{2} \theta_{t}\right)$ and for $C P$ odd decays $\frac{3}{4} \sin ^{2} \theta_{t}$. A 'cookbook' recipe for calculating $\cos \left(\theta_{t}\right)$ is given in Appendix A.2.

As $C P$-violation in the $B_{s}^{0}$ system is expected to be extremely small, the mass eigenstates $B_{s}^{H}$ and $B_{s}^{L}$ are nearly $C P$-eigenstates, with the heavy eigenstate $B_{s}^{H}$ being $\mathrm{CP}$ odd and the light eigenstate $B_{s}^{L} \mathrm{CP}$ even. Combining an angular analysis with a lifetime measurement can considerably improve the access to the width difference $\Delta \Gamma=\Gamma_{h}-\Gamma_{l}$ in the $B_{s}^{0}$ system [25].

In the Standard Model the fractional width difference is related to the $B_{s}^{0}$ mixing parameter $x_{s}=\frac{\Delta M_{B}}{\Gamma}$ by $[26]$

$$
x_{s}=-\frac{\Delta \Gamma}{\Gamma} \frac{2 m_{t}^{2}}{3 \pi m_{b}^{2}}\left(1-\frac{8 m_{c}^{2}}{3 m_{b}^{2}}\right)^{-1} h\left(\frac{m_{t}^{2}}{M_{W}^{2}}\right)
$$

where

$$
h(y)=1-\frac{3 y(1+y)}{4(1-y)^{2}}\left\{1+\frac{2 y}{1-y^{2}} \ln (y)\right\}
$$

with $y \equiv \frac{m_{t}^{2}}{M_{W}^{2}}$.

A precise measurement of $x_{s}$ and $\Delta \Gamma / \Gamma$ will be very sensitive to physics beyond the Standard Model [27]. New physics would result in $x_{s}$ being too large to measure while $\Delta \Gamma / \Gamma$ is accessible or vice versa. 


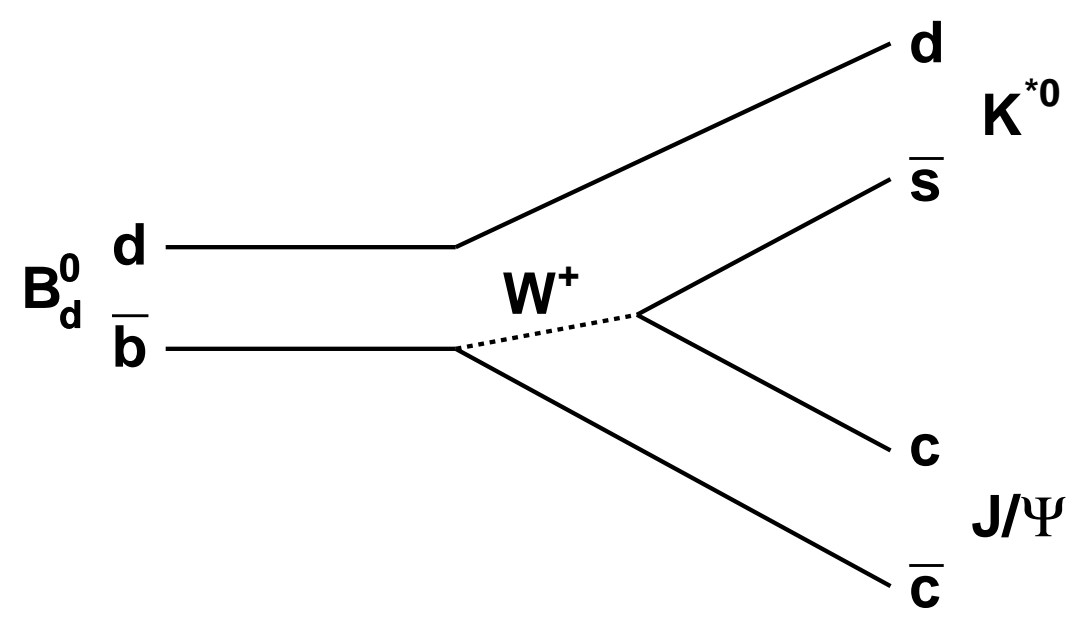

Figure 2.6: Tree level diagram for the decay $B_{d}^{0} \rightarrow J / \psi K^{* 0}$.

\subsection{The decay $B_{d}^{0} \rightarrow J / \psi\left(\rightarrow \mu^{+} \mu^{-}\right) K^{* 0}\left(\rightarrow K^{+} \pi^{-}\right)$}

This decay has similar decay kinematics to $B_{s}^{0} \rightarrow J / \psi \phi$ and both decays are often reconstructed together. A Feynman diagram of the decay is given in fig. 2.6.

As in the $B_{s}^{0}$ decay there is a four track vertex which can be used to measure the $B_{d}^{0}$ lifetime. But due to the larger width of the $K^{* 0}$ with respect to the $\phi$ and the ambiguity introduced by the fact that the $K^{* 0}$ decays into two different types of particles ${ }^{5}$, while the $\phi$ is reconstructed in the channel $K^{+} K^{-}$, the signal will not be as clean as in the $B_{s}^{0}$ channel.

The $K^{* 0}$ is a vector particle, so the theory of angular distributions as outlined before also applies here, with the $K^{* 0}$ taking on the role of the $\phi$. The different polarisations in this decay have been measured [24] to be

$$
\begin{aligned}
& \left|A_{0}\right|^{2}=0.59 \pm 0.06(\text { stat }) \pm 0.01 \text { (syst) } \\
& \left|A_{\perp}\right|^{2}=0.13_{-0.09}^{+0.12}(\text { stat }) \pm 0.06(\text { syst })
\end{aligned}
$$

The $K^{* 0}\left(\bar{K}^{* 0}\right)$ can also decay to $K^{0} \pi^{0}$. Together with an angular analysis this decay can be used to extract the CKM parameter $\sin 2 \beta$, similar to the decay $B_{d}^{0} \rightarrow J / \psi K_{S}^{0}$.

\footnotetext{
${ }^{5}$ This ambiguity of course only exists in a detector without particle identification, like DØ.
} 


\subsection{B-meson production at the Tevatron}

The dominant process for $b$-quark production at Tevatron energies [28] is gluongluon fusion ${ }^{6}$. To leading order the average transverse momentum of a $b$-quark is approximately equal to its mass $<p_{t}>\sim m_{b} \sim 4-5 \mathrm{GeV}$. The leading order QCD diagrams for $b$-quark production are given in fig. 2.7.

The next-to-leading order terms were originally considered corrections, but they turned out to be large. Yet even they could not explain the difference between the theoretically predicted cross-section and the cross-section measured by CDF and $\mathrm{D} \varnothing$ which is at least a factor of two larger [11].

A recent paper by R. Field [29] suggests that three different processes contribute to the $b$-quark production at the Tevatron: The first is flavour creation as described above. The second source of $b$-quarks is flavour excitation, which refers to the scattering of a $b(\bar{b})$-quark out of the initial state (i.e. the proton) into the final state by a gluon or a light quark. Thirdly, parton showers, where the $b$-quarks are produced during fragmentation rather than during the initial hard scattering, are also a significant source of $b$-quarks at the Tevatron. When taking into account the contributions of all three processes the theoretical predictions are in good agreement with the experimental results.

After a $b \bar{b}$ pair is produced it hadronizes to form pairs of $B$-mesons. The fractions of $B^{ \pm}, B_{d}^{0}, B_{s}^{0}$ and $b$-baryons produced have been measured to be [30]

$f_{u}: f_{d}: f_{s}: f_{\text {baryon }}=0.375 \pm 0.023: 0.375 \pm 0.023: 0.160 \pm 0.044: 0.090 \pm 0.029$

with the assumption that $f_{u}=f_{d}$. The ratio $f_{u} / f_{d}$ has been measured to be 0.84 \pm 0.16 .

\footnotetext{
${ }^{6}$ For top-quark production quark-antiquark annihilation prevails.
} 

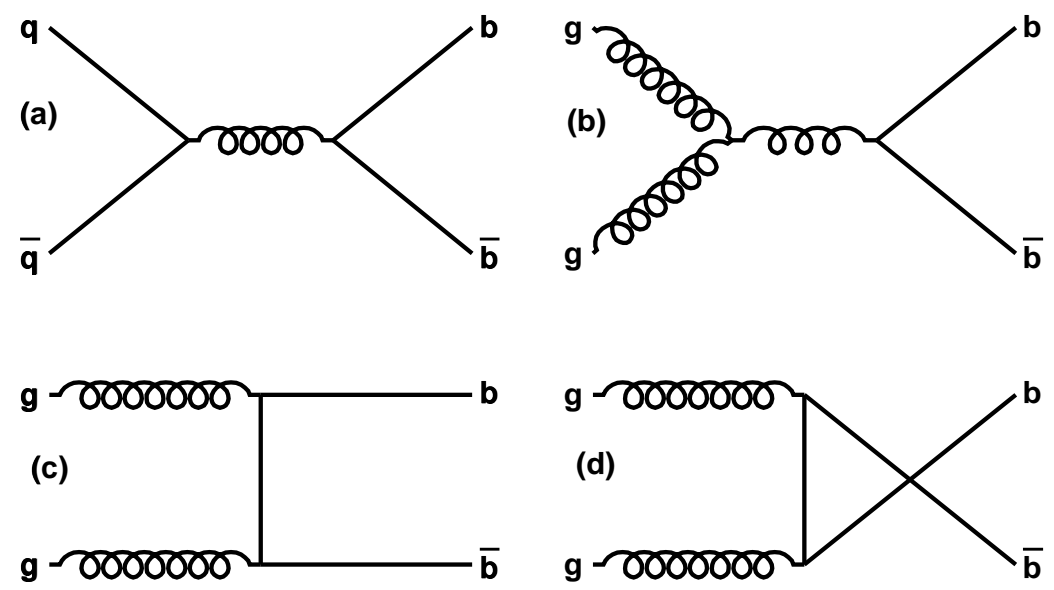

Figure 2.7: Leading order diagrams for $b$-quark production at the Tevatron: (a) quark-antiquark annihilation, (b)-(d) gluon-gluon fusion. 


\section{Chapter 3}

\section{The DØ Detector at the Tevatron}

This chapter gives a brief overview of the Tevatron and the various components of the $\mathrm{D} \emptyset$ detector.

\subsection{The Tevatron at Fermilab}

The Tevatron proton anti-proton collider, with a circumference of $6.4 \mathrm{~km}$, is the highest-energy particle collider currently operational. A schematic of the Fermilab accelerators is shown in fig. 3.1 and the main run parameters are listed in table 3.1. The upgraded accelerator started operating on March 1st 2001. The first collisions at $\mathrm{D} \varnothing$ occurred on April 3rd. The integrated luminosity is expected to be at least $2 \mathrm{fb}^{-1}$ over the first three years.

\subsection{The DØ Detector}

The $\mathrm{D} \varnothing$ experiment is a multi-purpose detector located at the Tevatron. During Run I between 1992 and 1996 a wide range of measurements were made at a centre of mass energy of $1.8 \mathrm{TeV}$, with the observation of the top quark in March 1995 as one of the highlights [31]. After the end of Run I, the Tevatron was upgraded to run at $\sqrt{s}=1.96 \mathrm{TeV}$ with an expected luminosity of $2 \times 10^{32} \mathrm{~cm}^{-2} \mathrm{~s}^{-1}$. While the 


\begin{tabular}{|l||c|c|c|}
\hline RUN & Run IIa $(36 \times 36)$ & Run IIa $(140 \times 103)$ & Run IIb $(140 \times 103)$ \\
\hline Energy $(p, \bar{p})$ & $980 \mathrm{GeV}$ & $980 \mathrm{GeV}$ & $980 \mathrm{GeV}$ \\
Protons/bunch & $2.7 \times 10^{11}$ & $2.7 \times 10^{11}$ & $2.7 \times 10^{11}$ \\
Antiprotons/bunch & $3.0 \times 10^{10}$ & $4.0 \times 10^{10}$ & $1.0 \times 10^{11}$ \\
Proton bunches & 36 & 140 & 140 \\
Antiproton bunches & 36 & 103 & 103 \\
Bunch Spacing & $396 \mathrm{~ns}$ & $132 \mathrm{~ns}$ & $132 \mathrm{~ns}$ \\
Typical Luminosity & $0.86 \times 10^{32} \mathrm{~cm}^{-2} \mathrm{~s}^{-1}$ & $2.1 \times 10^{32} \mathrm{~cm}^{-2} \mathrm{~s}^{-1}$ & $5.3 \times 10^{32} \mathrm{~cm}^{-2} \mathrm{~s}^{-1}$ \\
Integrated Luminosity & $17.3 \mathrm{pb}^{-1} /$ week & $42 \mathrm{pb}^{-1} /$ week & $105 \mathrm{pb}^{-1} /$ week \\
\hline
\end{tabular}

Table 3.1: Planned Tevatron parameters for Run II [32].

strengths of the original detector [33] were its finely segmented hermetic liquid argon calorimeter and large angle muon detection capability, the tracking suffered from the absence of a magnetic field in the central detector region. Therefore, in parallel to the accelerator, the $\mathrm{D} \varnothing$ detector was upgraded [34]. The main improvement was the introduction of a solenoid magnet providing a 2 Tesla field around a newly designed central tracking system (see fig. 3.2).

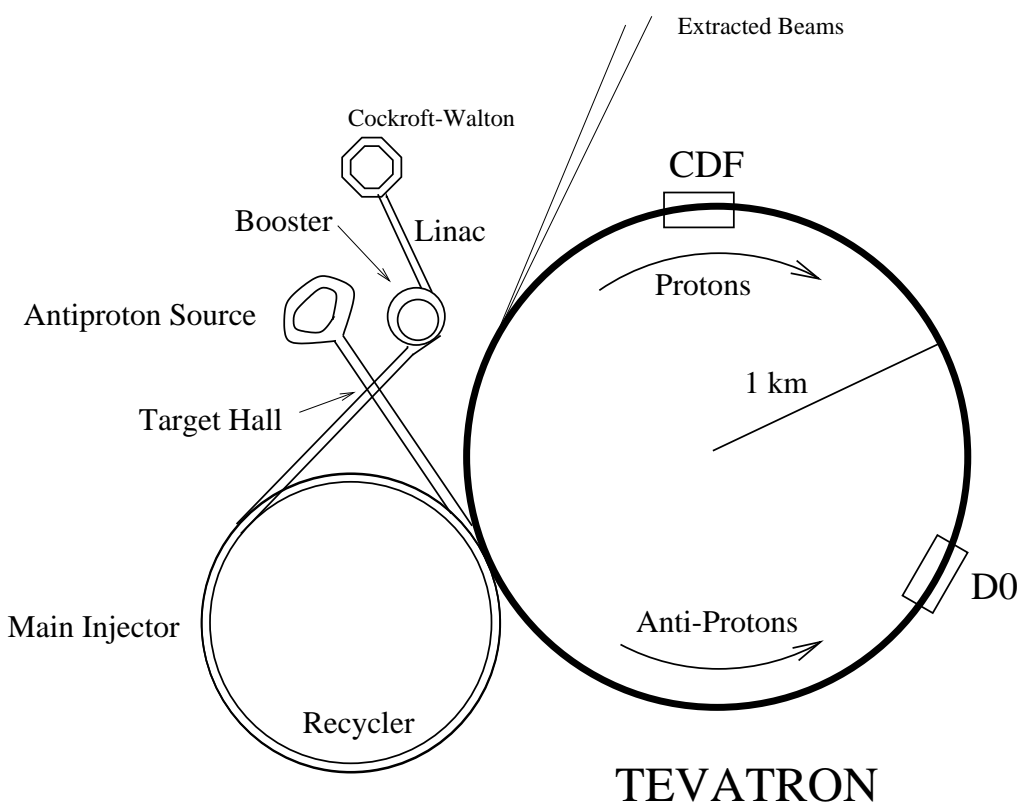

Figure 3.1: Fermilab accelerators schematic. 


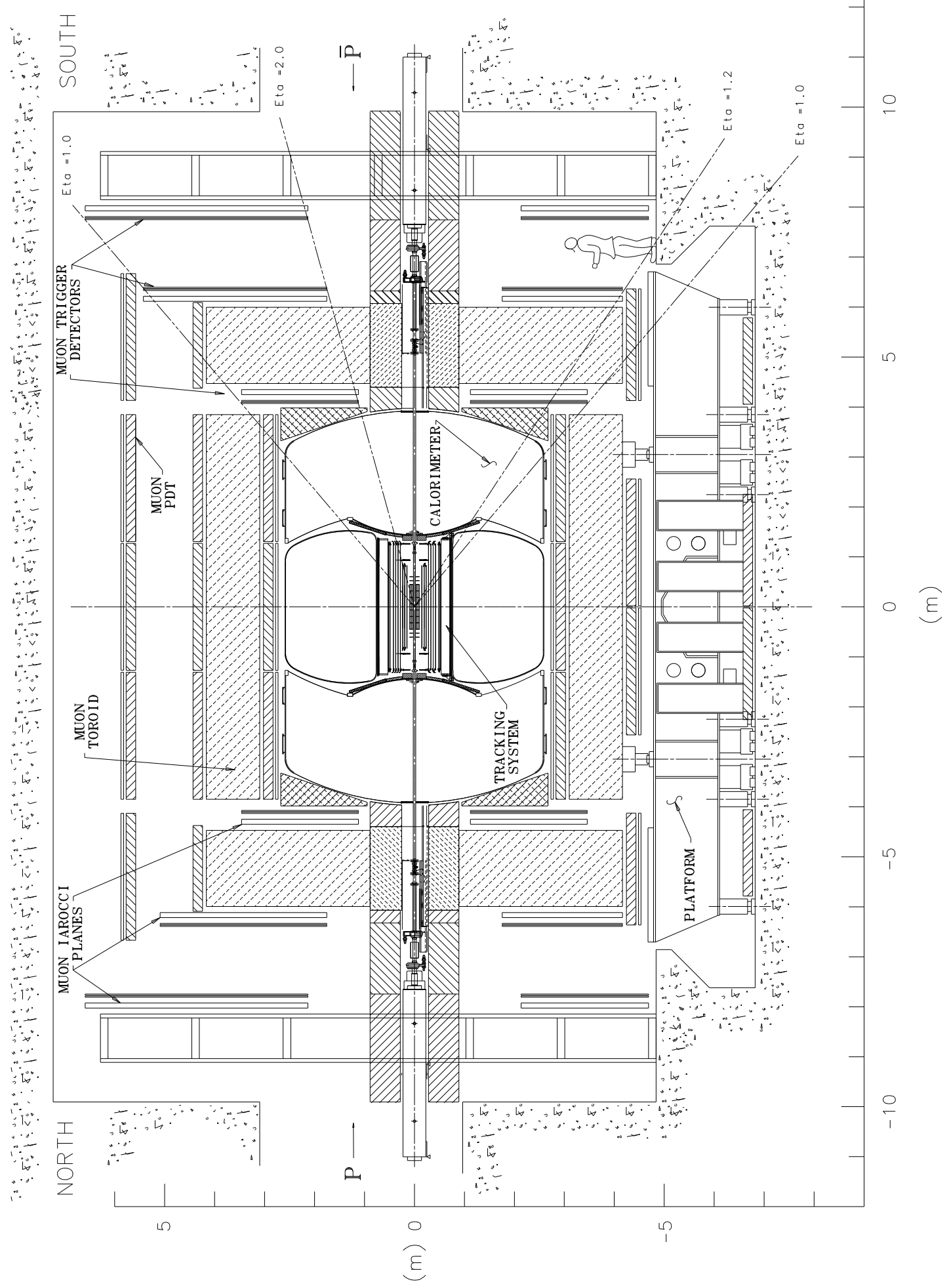

Figure 3.2: Side view of the DØ Run II detector [34]. 


\subsubsection{Coordinate System}

The following coordinate system is used throughout this thesis:

The positive $z$-axis is aligned along the beam in the direction of the protons. The $y$-axis points upwards and the $x$-axis away from the centre of the ring. When using spherical coordinates $(r, \phi, \theta)$, the angle $\theta$ is often replaced by pseudo-rapidity $\eta$ defined as

$$
\eta=-\ln \tan \frac{\theta}{2}
$$

which approaches the true rapidity,

$$
y=\frac{1}{2} \ln \frac{E+p_{z}}{E-p_{z}}
$$

in the limit $m \ll E$, where $m$ is the mass of a particle and $E$ its energy.

Transverse energy is defined analogously to the transverse momentum:

$$
p_{t}=p \sin \theta \quad E_{T}=E \sin \theta
$$

\subsubsection{Central Tracking System}

The introduction of a magnetic field into the $\mathrm{D} \emptyset$ detector was accompanied by the replacement of the inner tracking system. The new tracking system as shown in fig. 3.3 consists of an inner, high-resolution silicon microstrip tracker surrounded by a scintillating fibre tracker. It is designed to cover a large range of pseudo-rapidity $(|\eta| \leq 3)$ and will measure the momenta of charged particles. Other goals are electron identification, e $/ \pi$ rejection and $b$-tagging by the identification of displaced secondary vertices. The expected transverse momentum resolution [35] is $\Delta p_{t} / p_{t}=$ $\left(2+0.2 p_{t}\right) \%$. 


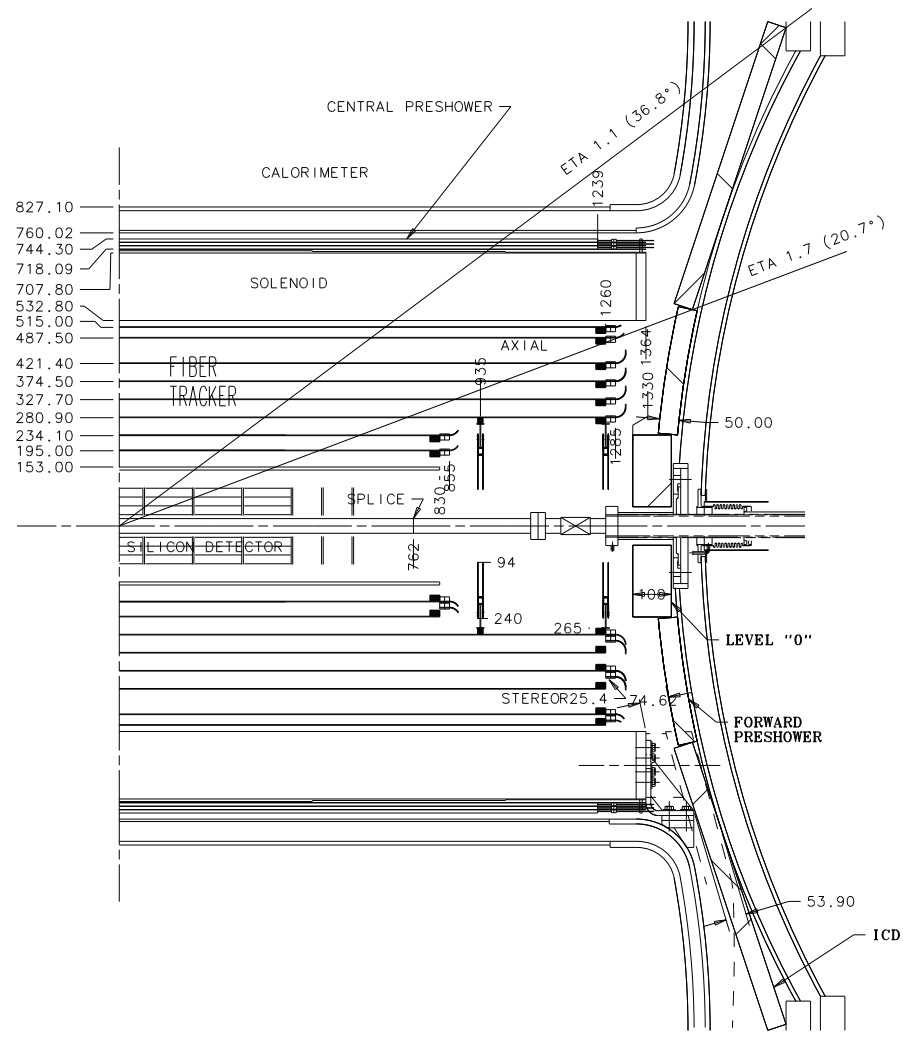

Figure 3.3: The DØ tracking system [34].

\section{Silicon Microstrip Tracker (SMT)}

Located closest to the interaction point is the silicon microstrip tracker. Its overall design is determined by the extended interaction point $\left(\sigma_{z}=25 \mathrm{~cm}\right)$ requiring a minimum length for the barrel section of $72 \mathrm{~cm}$. To provide optimal resolution all tracks should intersect the detector planes approximately perpendicularly. This leads to a hybrid system with barrel detectors measuring primarily the $r$ - $\phi$ coordinates, and disk detectors which measure $r$ - $\phi$ as well as $r-z$. The SMT consists of six $12.4 \mathrm{~cm}$ long barrel detectors containing eight layers of rectangular silicon microstrip detectors, here referred to as ladders. Layers $3,4,7$ and 8 of the barrel detectors are double-sided detectors at a stereo angle of $2^{\circ}$ relative to the beam-axis. In layers $1,2,5$ and 6 the stereo angle is $90^{\circ}$. Layers $1-4$ consist of 6 ladders, layers 5 to 8 of 12. In the outermost barrels the ladders on layers $1,2,5$ and 6 are single sided and no stereo information is available. 


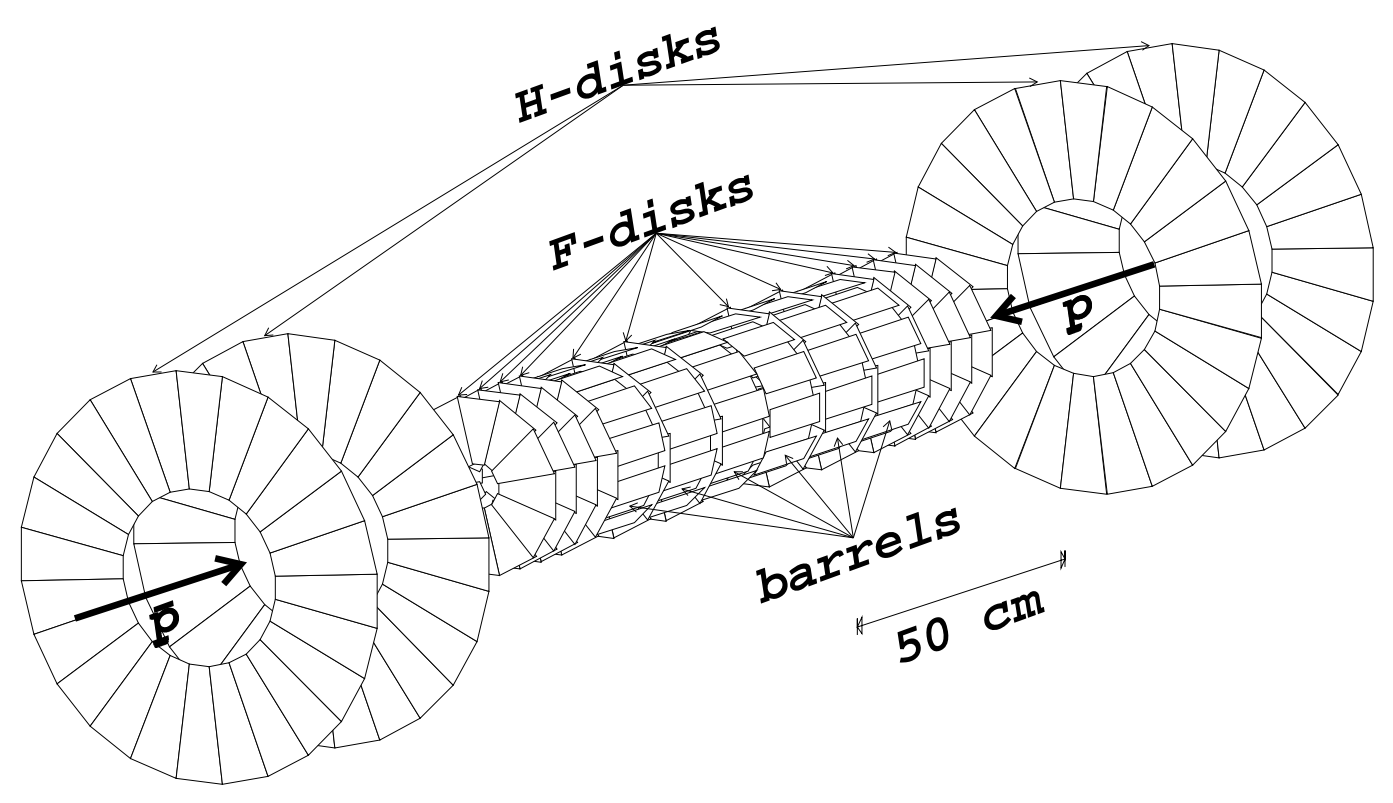

Figure 3.4: The Silicon Microstrip Tracker (picture taken from [36]).

There are 12 so-called 'F-disks' made from double sided detectors with $\pm 15^{\circ}$ stereo strips. Four of them are sandwiched between the barrel sections, the other eight being located at both ends of the barrel. Outside the F-disks, towards the end of the interaction region, there are two 'H-disks' on each side. They are made of two sets of single-sided wedge detectors with an effective stereo angle of $\pm 7.5^{\circ}$. F-disks have an outer radius of $12.5 \mathrm{~cm}, \mathrm{H}$-disks of $26 \mathrm{~cm}$. An overview of the microstrip detectors is given in table 3.2 .

The SMT is read out by 128 channel silicon readout chips, so-called SVXIIe chips [37]. They are designed to work with double sided detectors and are able to accept positive and negative currents as input signals. The SVXIIe chips are mounted on a high density interconnect (HDI). From the HDI the data pass via adaptor cards and interface boards to the sequencer board. An optical link connects the sequencer to the readout buffer. The whole SMT comprises $\sim 793000$ readout channels.

Test beam results show that a spatial resolution (track residuals) of better than 10 $\mu \mathrm{m}$ can be obtained [38]. The expected vertex resolution is $15-40 \mu \mathrm{m}$ in $r-\phi$ and $80-100 \mu \mathrm{m}$ in $z$. 
To minimize the effects of radiation damage [34] the operation temperature for the SMT is kept at $5-10^{\circ} \mathrm{C}$.

\begin{tabular}{|l||c|c|c|c|}
\hline Detector & View & Number of strips & Angle & Pitch \\
\hline Barrel (single sided) & 1 (p-side) & 384 & $0^{\circ}$ & $50 \mu \mathrm{m}$ \\
Barrel $90^{\circ}$ & 1 (p-side) & 384 & $0^{\circ}$ & $50 \mu \mathrm{m}$ \\
& 2 (n-side) & 768 & $90^{\circ}$ & $153.5 \mu \mathrm{m}$ \\
Barrel $2^{\circ}$ & 1 (p-side) & 640 & $0^{\circ}$ & $50 \mu \mathrm{m}$ \\
& 2 (n-side) & 512 & $2.004^{\circ}$ & $62.5 \mu \mathrm{m}$ \\
& & & & \\
F-Disk & 1 (p-side) & 1024 & $-15^{\circ}$ & $50 \mu \mathrm{m}$ \\
& 2 (n-side) & 768 & $15^{\circ}$ & $62.5 \mu \mathrm{m}$ \\
H-Disk & 1 (p-side) & 768 & $7.5^{\circ}$ & $79.3 \mu \mathrm{m}$ \\
& 2 (p-side) & 768 & $-7.5^{\circ}$ & $79.3 \mu \mathrm{m}$ \\
\hline
\end{tabular}

Table 3.2: SMT parameters. Note that the $n$-side of the ladders on the $90^{\circ}$ barrels are 'multiplexed': In these ladders two strips are connected to one readout channel and are read out simultaneously.

\section{Central Fibre Tracker (CFT)}

The central fibre tracker consists of 32 concentric barrel-shaped layers of scintillating fibres. These 32 layers are arranged in 16 doublet layers which are then grouped together in eight 'superlayers' at radii of approximately 19.5, 23.4, 28.1, 32.8, 37.5, 42.1, 48.8 and $51.4 \mathrm{~cm}$. The inner doublet in each superlayer is parallel to the beam axis, the outer one is oriented at alternating plus or minus $2.0^{\circ}-3.0^{\circ}$ stereo angles. The CFT comprises 76800 fibres. These scintillating fibres with a diameter of $835 \mu \mathrm{m}$ are made of a polystyrene core doped with $1 \%$ p-terphenyl (PTP) and 1500 ppm 3-hydroxyflavone. The PTP is used to increase the light yield, while the hydroxyflavone acts as a wavelength shifter to match the transmission properties of the polystyrene [39]. The core is surrounded by $15 \mu \mathrm{m}$ acrylic cladding, which in turn is covered by $15 \mu \mathrm{m}$ of fluoro-acrylic cladding in order to increase the light trapping [34]. The peak emission wavelength of these fibres is around $530 \mathrm{~nm}$.

Every fibre is mated to a 7-11 $\mathrm{m}$ long waveguide which pipes the scintillation light to a visible light photon counter (VLPC). VLPCs [40] are solid state photon detectors 
based on silicon diodes with an operating temperature of $\sim 10 \mathrm{~K}$. They have high gains (50 000 electrons per converted photon) and their quantum efficiency for visible light is $\sim 80 \%$.

The CFT is designed to provide track reconstruction within the range of $|\eta|<2.0$.

\subsubsection{Solenoid Magnet}

The central tracking system is surrounded by a superconducting solenoid [34]. This is a $2.8 \mathrm{~m}$ long two layer coil with a mean radius of $60 \mathrm{~cm}$, a field strength of 2 Tesla and a stored energy of 5 MJ. There is no specific flux return ${ }^{1}$. A uniformity of $\sin \theta \times \int B_{z} d l$ within $0.5 \%$ is achieved by applying an increased current density near the ends of the coil. The coil and its cryostat correspond to approximately 1.1 radiation lengths of material.

\subsubsection{Preshower Detectors}

\section{Central Preshower}

Located in the $51 \mathrm{~mm}$ gap between the solenoid and the central calorimeter cryostat, the central preshower detector covers a region of $|\eta|<1.2$. Its main purpose is to enhance the electron identification and to correct for the effects of the tracking system, solenoid and lead absorber in the reconstruction of electromagnetic energy in the calorimeter. The solenoid and the tapered lead absorber placed in front of the detector correspond to about two radiation lengths of material. Studies [42] suggest that the energy in the central preshower must be known within $10 \%$ to reach the overall electromagnetic energy resolution of Run I.

The central preshower consists of triangular scintillating strips arranged in one inner axial layer and two outer stereo layers at an angle of $\sim \pm 23^{\circ}$. Each strip has a base of $7.1 \mathrm{~mm}$ with a $1 \mathrm{~mm}$ hole in the centre. The readout occurs via wavelength-shifting fibres passing through this hole. Near the end of the strips the wavelength-shifting

\footnotetext{
${ }^{1}$ Most of the flux generated by the solenoid returns in the space between the cryostat and the muon system. The magnetic interaction between the solenoid and the muon system was found to be negligible [41].
} 
fibres are connected to clear waveguide fibres. These lead to visible light photon counters (VLPCs) under the detector platform.

\section{Forward Preshower}

Two forward preshower detectors are mounted on the inner surface of each end calorimeter. Their design is very similar to the central preshower detectors and they cover the pseudo-rapidity range $1.4<|\eta|<2.5$. A layer of lead absorber is sandwiched between two scintillator planes. The inner scintillators act as detectors for minimum ionising particles, except between $1.4<|\eta|<1.6$, where particles traverse the magnet solenoid. In this region the inner layer is not necessary as particles are likely to shower upstream of the forward preshower.

\subsubsection{Calorimeter}

The liquid-argon calorimeter [33] provides energy measurements for electrons, photons and jets. It remains unchanged from Run I, but the much lower bunch crossing time of 396(132) ns in Run II requires an upgrade of its front-end electronics. The former peak sampling time of $2.2 \mu$ s will be reduced to $400 \mathrm{~ns}$, mainly by decreasing the effective integration time and reducing the intrinsic noise of the pre-amplifier [34].

The calorimeter as shown in fig. 3.5 is divided into a central calorimeter $(|\eta| \leq 1)$ and two end calorimeters to cover the region $1 \leq|\eta| \leq 4$. Both calorimeters contain three basic types of modules: An electromagnetic section with thin (3 or 4 $\mathrm{mm}$ ) nearly pure depleted uranium plates, fine hadronic modules with $6 \mathrm{~mm}$ thick uranium-niobium(2\%) alloy plates and coarse hadronic sections. These sections consist of $46.5 \mathrm{~mm}$ thick plates, made from copper for the central calorimeter and stainless steel in the end calorimeters. There are four separate layers for the electromagnetic modules, three for the fine hadronic and one or three for the coarse hadronic modules. These layers are used to measure the longitudinal shower shape in order to distinguish between electrons and hadrons. The energy resolution is $14 \% / \sqrt{E}$ for electromagnetic showers and $80 \% / \sqrt{E}$ for hadronic jets. 
The smallest unit of the calorimeter is a readout cell, typically covering $0.1 \times 0.1$ in $\eta$ and $\phi$, except for the third layer in the electromagnetic modules, where the cells cover $0.05 \times 0.05$.

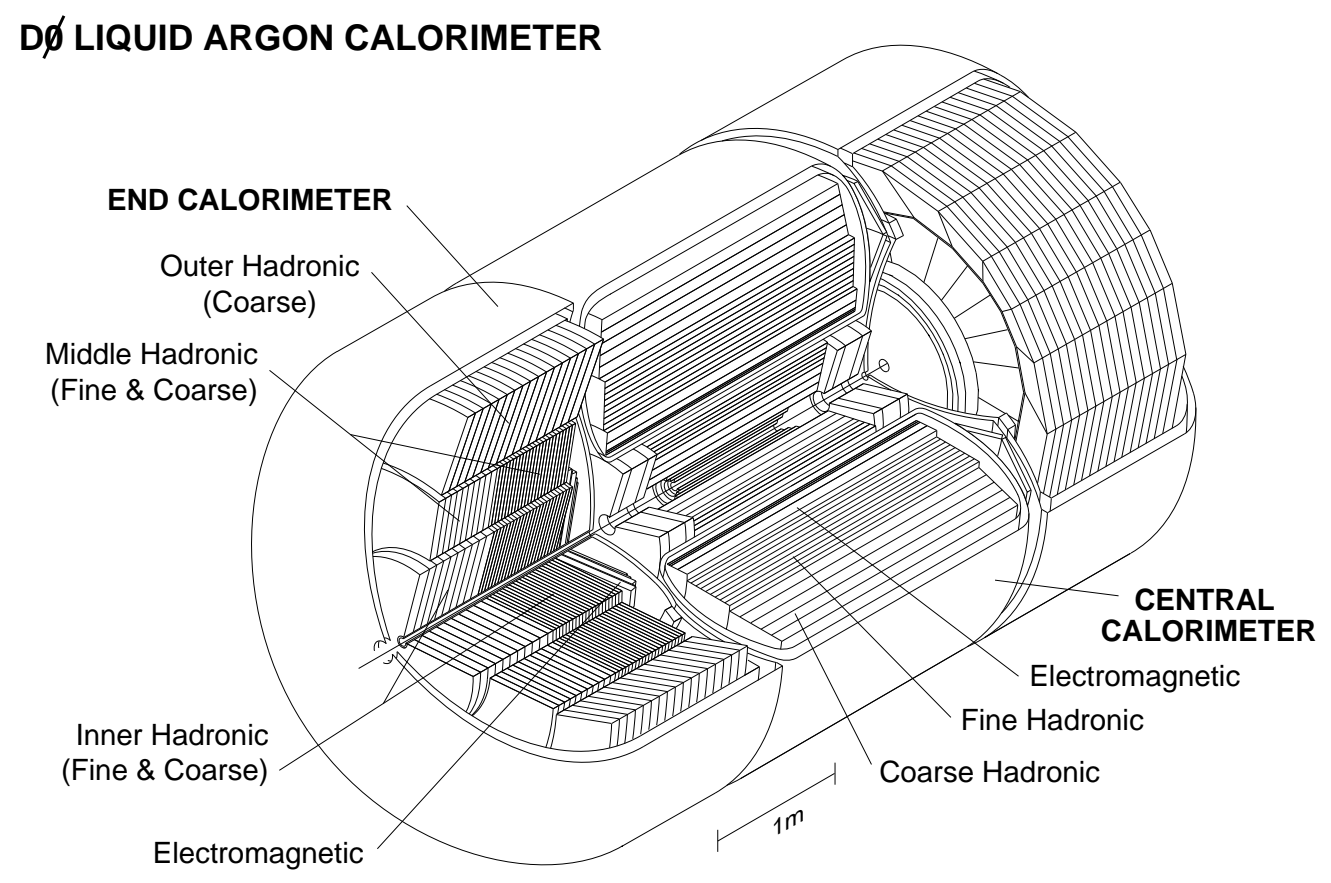

Figure 3.5: The liquid-argon calorimeter [33].

\subsubsection{Intercryostat Detector}

The transition region between the central and the end calorimeters $(0.8 \leq|\eta| \leq$ 1.4) contains a large amount of uninstrumented material, mainly support structures for the calorimeter and the cryostat walls. To correct for energy deposited in this area, a so-called intercryostat detector has been installed [33], [34]. It consists of a single layer of 384 scintillating tiles of size $\Delta \eta=\Delta \phi=0.1$ to match the liquid-argon calorimeter cells. The tiles are mounted on the front surface of the end calorimeters. The light signals are picked up by wavelength-shifting fibres in the tiles and then transported via clear fibre ribbon cables towards the phototubes outside the magnetic field. 


\subsubsection{Muon System}

In order to study low cross-section processes in a wide range of $p_{t}$ the muon system must cover the region $|\eta|<2$ and provide an efficient unprescaled trigger. Good muon coverage is essential for a range of $b$-physics topics, which require the reconstruction of a $J / \psi$ meson.

The muon detectors [34] are divided into two main parts: The Wide Angle Muon System covering the region $|\eta|<1$ and the Forward Muon System covering $1<|\eta|<2$.

\section{Wide Angle Muon System (WAMUS)}

From the interaction region outwards the WAMUS consists of a layer of scintillation counters located between the calorimeter and the first layer of proportional drift chambers (PDT). The scintillators are needed for triggering, as the maximum drift time in the PDT of 750 ns exceeds the bunch spacing in Run II (396 or $132 \mathrm{~ns}$ ). Located after the first layer of PDTs is a toroidal magnet providing a 1.6 Tesla field. Immediately after the magnet follows a second layer of PDTs and after a gap a third PDT layer. The hit efficiency [43] for the PDTs is 99\%. Mounted on the outside of the third PDT layer is another layer of scintillation counters. Scintillation counters can also be found on the second layer, at the bottom of the detector. The rectangular drift chambers are constructed from extruded aluminium. Their anode wires are made from gold-plated tungsten. The drift distance resolution is $\sim 500$ $\mu \mathrm{m}$. The scintillation counters are made from plastic scintillator and wavelength shifting fibres.

\section{Forward Muon System (FAMUS)}

Analogous to the WAMUS, the Forward Muon System consist of three layers of drift chambers for track reconstruction and three layers of scintillation counters for triggering. During Run I, the PDTs in the forward region suffered from radiation induced build-up of material. To avoid time-consuming cleaning procedures, the PDTs in the Forward Muon System will be replaced by so-called Mini Drift Tubes (MDT) which studies have shown to be radiation-hard [44]. Their hit efficiency [43] 
is $>97 \%$. These tubes are made from commercially available aluminium extruded combs. An individual tube contains 8 cells, each with a $9.4 \times 9.4 \mathrm{~mm}^{2}$ internal cross-section with $50 \mu \mathrm{m} \mathrm{W-Au}$ anode wires in the centre. The internal walls are coated with stainless steel foil to form the cathode. When filled with a fast gas mixture $\left(\mathrm{CF}_{4}(90 \%)+\mathrm{CH}_{4}(10 \%)\right)$ the maximum electron drift time is $60 \mathrm{~ns}$ and so is considerably shorter than a bunch spacing time of $132 \mathrm{~ns}$. The coordinate resolution is less than $1 \mathrm{~mm}$.

\subsection{Trigger}

The planned bunch-crossing time of 396 or 132 ns necessitates an upgrade of the trigger systems [45]. The trigger system is needed to reduce the number of background events and select physically interesting events to be written to tape. The data rate is reduced in three steps: Level 1 and 2 are hardware triggers, while the Level 3 trigger is software based.

\begin{tabular}{|l|c|c|}
\hline Trigger & accept rate & time available \\
\hline Events & $7.6 \mathrm{MHz}$ & \\
Level 1 & $10 \mathrm{kHz}$ & $4.2 \mu \mathrm{s}$ \\
Level 2 & $1 \mathrm{kHz}$ & $100 \mu \mathrm{s}$ \\
Level 3 & $20-50 \mathrm{~Hz}$ & $100-150 \mathrm{~ms}$ \\
\hline
\end{tabular}

Table 3.3: Trigger rates for Run II. The accept rate is the number of events per second that are passed on to the next stage. In the case of the Level 3 trigger it is the number of events written to tape.

\subsubsection{Level 1 and Level 2 Trigger}

The Level 1 trigger uses data from the calorimeter, the preshower detectors, the central fibre tracker and the muon MDTs and scintillators. It uses Field Programmable Gate Arrays (FPGAs) to test if any of the 128 Level 1 trigger bits have been set. 
In this case the Level 1 framework issues an accept and the data are digitized and moved to a series of 16 event buffers.

The Level 2 trigger is the first stage to match information from different detector subsystems. The data are processed via FPGAs and microprocessor cards. At a later stage it is planned to introduce a silicon track trigger [36] into Level 2 to enhance the trigger capabilities for long-lived particles e.g. $b$-quarks.

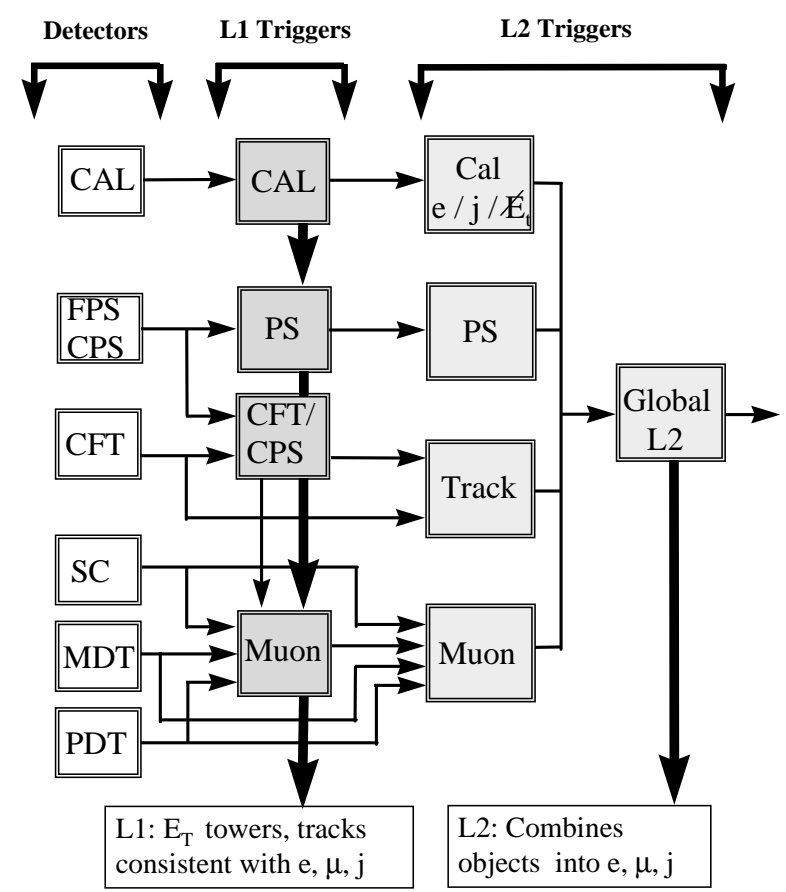

Figure 3.6: Level 1 and 2 trigger overview. The information flows from left to right.

\subsubsection{Level 3 Trigger}

The Level 3 trigger refines the physics objects (tracks, clusters, etc.) created in the Level 2 trigger and performs a limited reconstruction of the event. The Level 3 trigger software runs on a farm of standard PCs using the Linux operating system. Each processor of the farm, a so-called 'Level 3 node' runs an independent instance of the Level 3 filtering software and processes a complete event.

The Level 3 software consists of four major components [46]: Tools, filters, filter scripts and ScriptRunner. 
There are four different types of tools:

- Unpacking tools are the only tools that have access to the raw data. They are responsible for unpacking regions of interest as indicated by the Level 2 trigger.

- Data tools perform a partial reconstruction of the event (e.g. clustering, vertexing).

- Physics tools identify physics objects like electrons, jets, muons and others.

- Trigger tools supply general functionality like prescales or error handling.

A filter uses physics objects produced by the tools and checks against a given set of constraints, defined in the trigger list. A filter script corresponds to a Level 3 filtering trigger bit and combines the results of several filters. The top level software of the Level 3 trigger is ScriptRunner. For each fired Level 1/2 bit, ScriptRunner executes all associated filter scripts. Once the filter script is run the event is classed as passed, failed, unbiased or in error.

\subsection{Software}

The DØ software for Run II is being developed using the Concurrent Versions System (CVS) [47]. The software is divided into packages most of which are part of the DØ framework [48]. All DØsoftware, except for most of the Monte Carlo generators, is written in $\mathrm{C}++$.

Each package is a collection of classes which perform a limited task. For example the package 13fsmtunptool contains the code used to unpack the SMT data for the Level 3 trigger.

Parameters which cannot or should not be hard coded are stored in rcp (run control parameter) files. They are read in when required at execution time.

The format to store data is called a 'chunk'. All chunks inherit from a common base 
class and can be accessed via predefined methods. Each chunk stores a certain type of data. Examples are the SimSMTHitChunk which stores all the simulated GEANT [49] hits in the SMT or the GTrackChunk which contains all reconstructed global tracks. 


\section{Chapter 4}

\section{Clustering for the Silicon Microstrip Tracker}

\subsection{SMT clustering for the Level 3 trigger}

Fast and efficient cluster finding is essential for the Level 3 trigger. The cluster finding is divided into two distinct steps:

First, clusters are found for each side of a wafer. This is done in parallel to the unpacking [50] in the $13 \mathrm{f}$ smtunptool package. While this is a one-dimensional measurement, the clusters are (somewhat misleadingly) referred to as two-dimensional clusters, due to the fact that the axial clusters which are accessed by other parts of the trigger software are described by their $x$ and $y$ coordinates at this stage. Three-dimensional clusters refer to clusters where all three coordinates have been determined from measurements, rather than one or more of them being set to an average value. In the second step, these two-dimensional clusters are combined to form three-dimensional clusters in the $13 \mathrm{fsmt}$ cluster [51] package.

The chapter starts with a short description of the coordinate systems used. Then the algorithms for both steps of the clustering are presented separately, followed by the evaluation of the tools based on Monte Carlo data.

In order to reduce the amount of fake clusters in real data, I investigated using a 
parametrized pedestal subtraction instead of the initially used flat pedestals. This work is described in section 4.3 .

At the end of the chapter the results for testing the tools on cosmics are presented.

\subsubsection{Coordinate systems}

\section{Local coordinates}

Each ladder or wedge has its own local coordinate system [52], [53]. Its origin is located at the centre of the ladder or wedge. The local $z$-axis points towards the SVX chips and the local $y$-axis is directed from the first side $\left(\right.$ view $\left.^{1}=1\right)$ of the ladder to the second. The $x$-axis is oriented to form a right handed coordinate system.

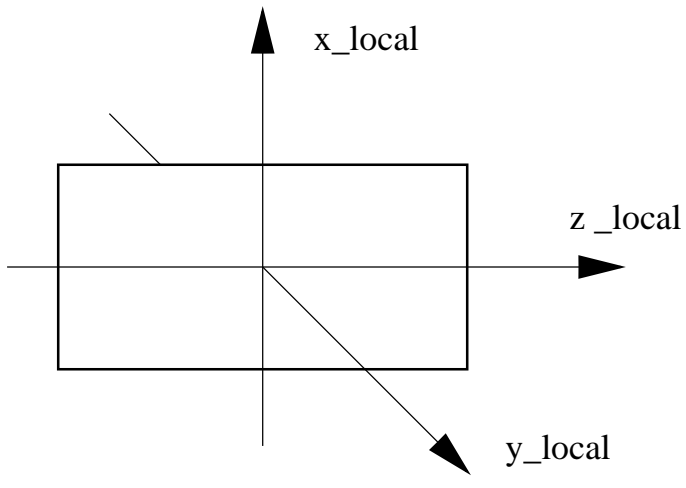

Ladder

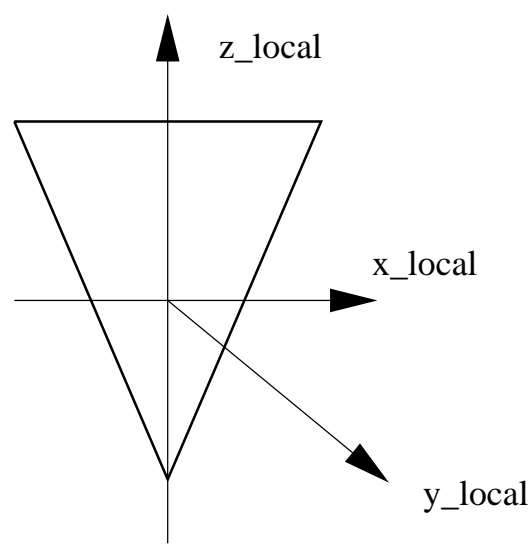

Wedge

Figure 4.1: The local coordinate system for ladders and wedges.

When grouping adjacent strips into clusters on one side of a ladder, the cluster can be described by a single position measurement, the cluster centroid. It is measured along a line perpendicular to the orientation of the strips. On a double sided detector there is a different angle on each side. Given a centroid position on each side the

\footnotetext{
${ }^{1}$ See table 3.2 .
} 


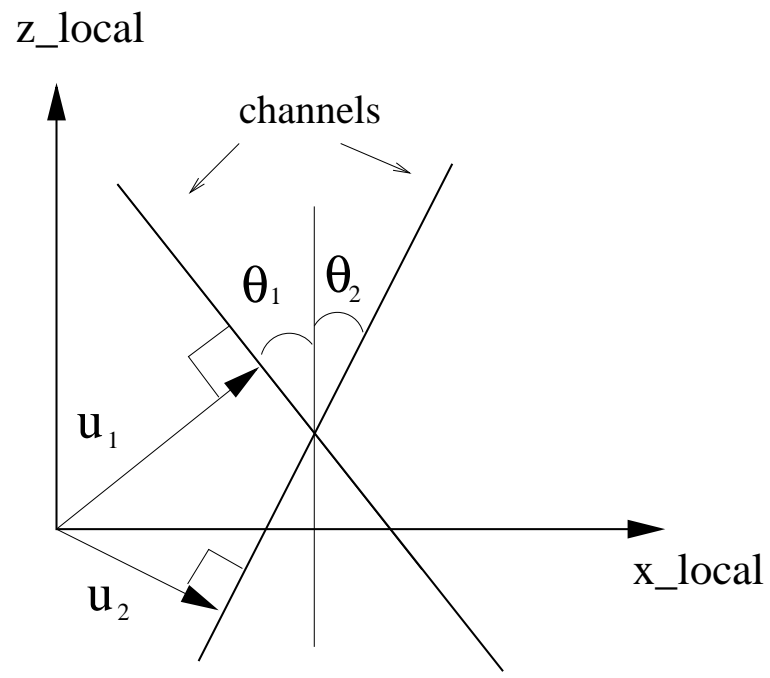

Figure 4.2: Illustration for equation 4.1.

point where they cross (fig. 4.2) is given by

$$
\begin{aligned}
& x_{L}=\frac{u_{1} \sin \theta_{2}-u_{2} \sin \theta_{1}}{\cos \theta_{1} \sin \theta_{2}-\sin \theta_{1} \cos \theta_{2}} \\
& z_{L}=\frac{u_{1} \cos \theta_{2}-u_{2} \cos \theta_{1}}{\cos \theta_{1} \sin \theta_{2}-\sin \theta_{1} \cos \theta_{2}}
\end{aligned}
$$

where $u_{1}, u_{2}$ are the centroid positions and $\theta_{1}, \theta_{2}$ are the angles on sides 1 and 2 respectively. On barrel ladders $\theta_{1}=0$ and this formula reduces to:

$$
\begin{aligned}
& x_{L}=u_{1} \\
& z_{L}=\frac{u_{1} \cos \theta_{2}-u_{2}}{\sin \theta_{2}}
\end{aligned}
$$

and on $90^{\circ}$ stereo ladders it becomes

$$
z_{L}=-u_{2}
$$

\section{Global coordinates}

Fig. 4.3 shows the relative orientation of the SMT detector to the global coordinate system. 
Due to time constraints the Level 3 software does not use the full geometry system. Instead it assumes that the ladders and wedges are flat and uses a simple linear transformation between local $\left(x_{L}, y_{L}, z_{L}\right)$ and global $\left(x_{G}, y_{G}, z_{G}\right)$ coordinates:

$$
\left(\begin{array}{c}
x_{G} \\
y_{G} \\
z_{G}
\end{array}\right)=\vec{r}_{0}+x_{L} \vec{r}_{1}+z_{L} \vec{r}_{2}
$$

The vector $\vec{r}_{0}$ points from the global origin to the centre of the ladder/wedge, $\vec{r}_{1}$ is the global coordinate vector corresponding to the vector $(1,0,0)$ in the local ladder/wedge coordinates, while $\vec{r}_{2}$ corresponds to $(0,0,1)$.

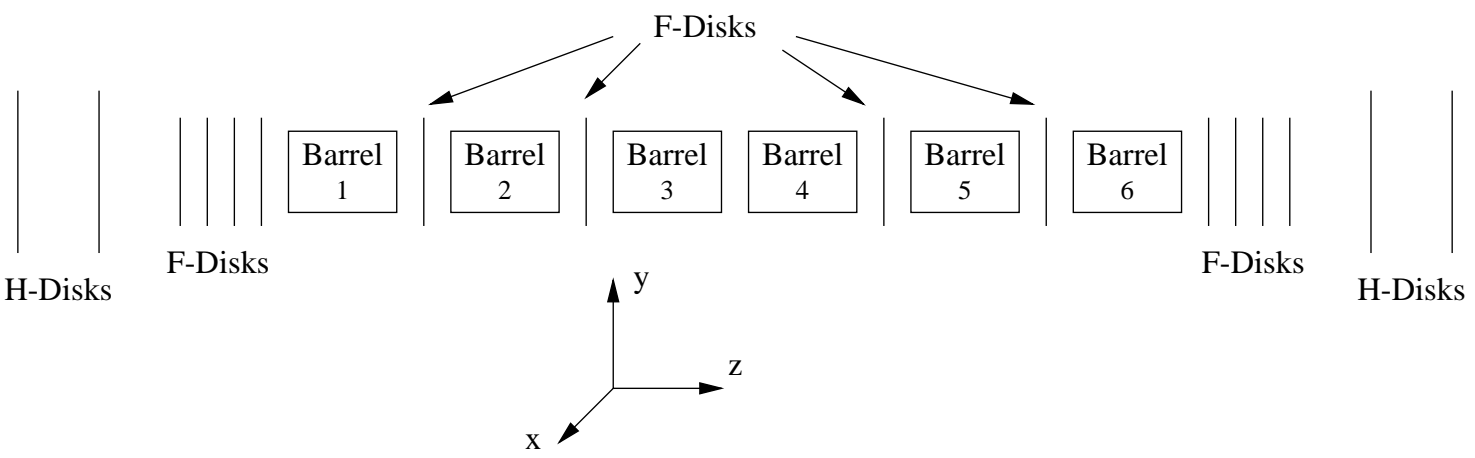

Figure 4.3: The global coordinate system. The proton beam is along the positive $z$-axis. The $x$-axis points away from the centre of the ring.

\subsubsection{Two-dimensional clustering}

The unpacking [50] of the data and the first step of the clustering are performed simultaneously to avoid copying large amounts of data to and from memory. The algorithm for forming the clusters is outlined below:

Only strips with an energy above a set threshold are used. For each new strip, its position address is checked. If it is on the same side on the same detector element as the current cluster and if there is a gap of no more than one strip between it and this cluster then the strip is added to the current cluster. If not or if it is the first strip 
unpacked then it marks the start of a new cluster and the geometry calculations for the existing cluster (if any) are done and it is stored in the list of clusters.

The geometry calculations and storing of the final cluster must be explicitly forced when all the strips have been read out.

As the readout of the stereo side of the $90^{\circ}$ detectors is multiplexed ${ }^{2}$, each of the stereo clusters on these ladders is duplicated, with an offset of $\sim 6 \mathrm{~cm}$. The position of the cluster is given by the pulse-height weighted average of the strips:

$$
\bar{n}=\frac{\sum n_{i} w_{i}}{\sum w_{i}}
$$

where $n_{i}$ is the strip number of the $i$ th strip and $w_{i}$ is the deposited energy in that strip. The weighted average is converted into a centroid position by using

$$
u=u_{1}+(\bar{n}-1) p
$$

where $u_{1}$ is the position of the centre of the first strip and $p$ is the pitch of the strips. The -1 is needed because the strip numbering starts at 1 . The next step is transforming the centroid position $u$ into local coordinates. For axial barrel clusters this is

$$
\begin{aligned}
x_{L} & =u \\
z_{L} & =0
\end{aligned}
$$

and for $90^{\circ}$ barrel clusters

$$
\begin{aligned}
& x_{L}=0 \\
& z_{L}=-u
\end{aligned}
$$

For the $2^{\circ}$ barrel and disk detectors the two-dimensional clusters provide no useful information, except their centroid position which is used to form three-dimensional clusters. The last step is to convert the local coordinates into global coordinates by

\footnotetext{
${ }^{2}$ Each SVX channel is connected to two strips which are separated by approximately $6 \mathrm{~cm}$ along the $z$-axis. It is impossible to distinguish from which of the two strips a signal originated.
} 
using equation 4.4 .

\subsubsection{Three-dimensional clustering}

The three-dimensional clustering takes place in the $13 \mathrm{f}$ smtcluster package. It uses the two-dimensional disk and barrel clusters from 13fsmtunptool as input. They are required to have been sorted by ladder or wedge. The algorithm for the barrels and disks follows the same principle. For every ladder/wedge all combinations of $2 \mathrm{~d}$ clusters which result in a $3 \mathrm{~d}$ cluster consistent with the hardware ${ }^{3}$ are taken into account. If there are only clusters on one side of a ladder these are kept as well (pseudo-3d clusters). Their missing coordinates are set to the values at the centre of the ladder.

For barrel clusters the global coordinates for each hit are taken directly from the unpack tool, except for the $z$-coordinate of clusters on layers with a $2^{\circ}$ stereo angle, where equation 4.2 is used. For disk clusters the local $x$ and $z$ coordinates are calculated using equation 4.1 .

The transformation from local into global coordinates is the same as in $13 f$ smtunptool.

\section{Ghost killing}

Ghosts are combinations of $2 \mathrm{~d}$ hits which originate from two different tracks. The $3 \mathrm{~d}$ clusters are grouped in collections: All clusters which derive from the same cluster on the axial (first) side of a ladder belong to one collection. This enables the elimination of ghosts once one cluster of a collection is identified as valid. The collection number for pseudo-3d clusters is set to zero.

For real hits the energy deposited should be approximately the same for both sides of the detector. Therefore the ratio

$$
\frac{\left|e_{1}-e_{2}\right|}{e_{1}+e_{2}}
$$

\footnotetext{
${ }^{3}$ For the disks and for ladders with a $2^{\circ}$ stereo angle, it is possible that $z_{\text {local }}$ calculated according to equation 4.2 results in a value that is greater than the limits of the ladder.
} 


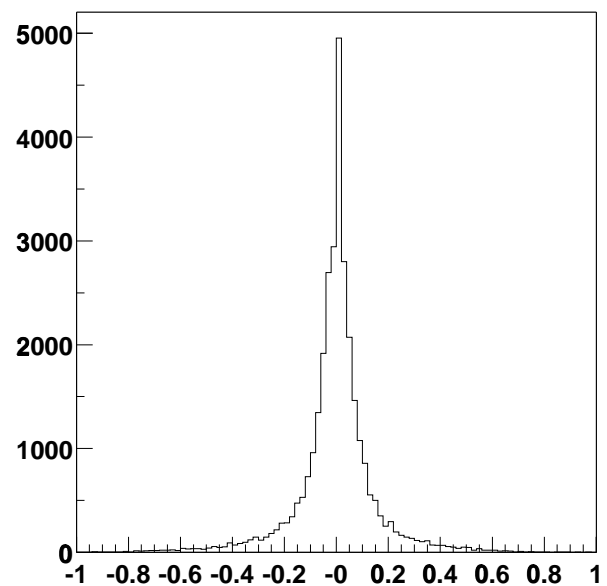

(a)

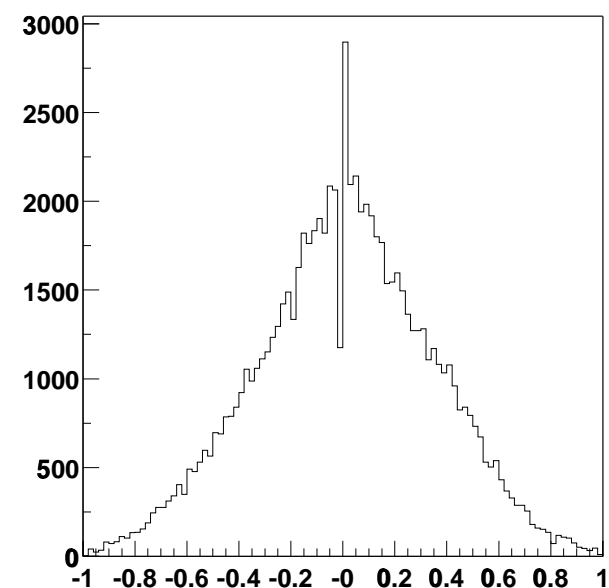

(b)

Figure 4.4: Example distributions of the energy ratio $\frac{e 1-e 2}{e 1+e 2}$ for real hits (a) and ghosts (b). Note that ghost-killing will not reduce the number of fake clusters due to multiplexing in the $90^{\circ}$ stereo layers. The spike in (b) is due to rounding errors.

where $e_{1}, e_{2}$ are the energies on both sides, should be close to zero. It is possible to reject clusters where this ratio is above a certain threshold. This cut is controlled by an $\mathrm{rcp}$ parameter. The default setting is 1 , i.e. no ghost-killing by $\mathrm{dE} / \mathrm{dx}$. While ghost-killing works on $\mathrm{MC}$ data (see fig. 4.4) it has yet to be seen whether the calibration will be sufficient to allow ghost-killing in real data. 


\subsection{Evaluation of the software}

\subsubsection{Monte Carlo matching}

The Monte Carlo (MC) data are stored as SimTkHits [54] which are an interface to the underlying GEANT [49] hits. GEANT propagates a particle in discrete steps through the detector. As the stepsize exceeds the thickness of the strips it is necessary to calculate the position of a MC hit at the middle of a strip (i.e. at $y_{\text {local }}$ $=0)$. This is done using the following equation:

$$
\begin{aligned}
& x_{\text {global }}^{m c}=x_{\text {global }}^{\text {in }}+y_{\text {local }}^{\text {in }} * \frac{x_{\text {global }}^{\text {out }}-x_{\text {global }}^{\text {in }}}{y_{\text {local }}^{\text {in }}-y_{\text {local }}^{\text {out }}} \\
& y_{\text {global }}^{m c}=y_{\text {global }}^{\text {in }}+y_{\text {local }}^{\text {in }} * \frac{y_{\text {global }}^{\text {out }}-y_{\text {global }}^{\text {in }}}{y_{\text {local }}^{\text {in }}-y_{\text {local }}^{\text {out }}} \\
& z_{\text {global }}^{m c}=z_{\text {global }}^{\text {in }}+y_{\text {local }}^{\text {in }} * \frac{z_{\text {global }}^{\text {out }}-z_{\text {global }}^{\text {in }}}{y_{\text {local }}^{\text {in }}-y_{\text {local }}^{\text {out }}}
\end{aligned}
$$

The index in refers to the beginning of a GEANT step, out to the end. The coordinates $x_{\text {global }}^{m c}, y_{\text {global }}^{m c}, z_{\text {global }}^{m c}$ are the global coordinates at the middle of the strip. For (almost) every particle GEANT creates a shower inside the material. Some of these shower particles create MC hits. These hits are usually not visible in the detector because they deposit only a small amount of charge. As they are very close to the primary particle position, the charge is superimposed. This means they fire exactly the same set of strips as the original particle (at least in the simulation). The task is to find hits suitable for tracking and not the position for every single particle from a shower within one silicon layer. Characteristically a shower hit is indicated by a step size less than the thickness of the silicon detector $(0.0275 \mathrm{~cm})$. These hits are not used in the MC matching.

Unless indicated otherwise the Monte Carlo studies are based on $t \bar{t}$ events with a typical track multiplicity of 50-60 tracks per event. 


\subsubsection{Results: Resolutions, Efficiencies and Timing}

Track and vertex finding in the Level 3 trigger depend on properly reconstructed SMT clusters. Therefore considerable effort has gone into determining the properties of these clusters. The results are summarised in this section.

Figs. 4.5 and 4.6 show the resolution for barrel clusters and figs. 4.7 and 4.8 their efficiencies. The resolutions are determined by fitting a Gaussian to the central peak of the distributions. The tails of these distributions have been ignored as only a partial reconstruction of the event is performed at Level 3 and the trigger efficiencies will not be significantly effected by the non-Gaussian tails.

It is unclear if the disks will be used in the trigger, but extending the code to include the disks is straightforward and the achieved resolutions are shown in figs 4.9 and 4.10.

Definitions of terms used are given below. A summary of all resolutions is given in table 4.1 .

\section{Definitions}

Matched: In order to be considered matched the distance between the MC hit and the corresponding reconstructed hit has to be less than approximately $3 \sigma$, where $\sigma$ is the resolution of the distance between the $\mathrm{MC}$ and the reconstructed hits. The exact values for each case are given with the histograms.

Efficiency is defined as the ratio of all MC hits which could be matched divided by all MC hits with the exception of shower hits as described in section 4.2.1.

Misidentification is defined as the number of unmatched reconstructed clusters divided by the total number of reconstructed clusters. 


\section{Barrels: Resolutions}

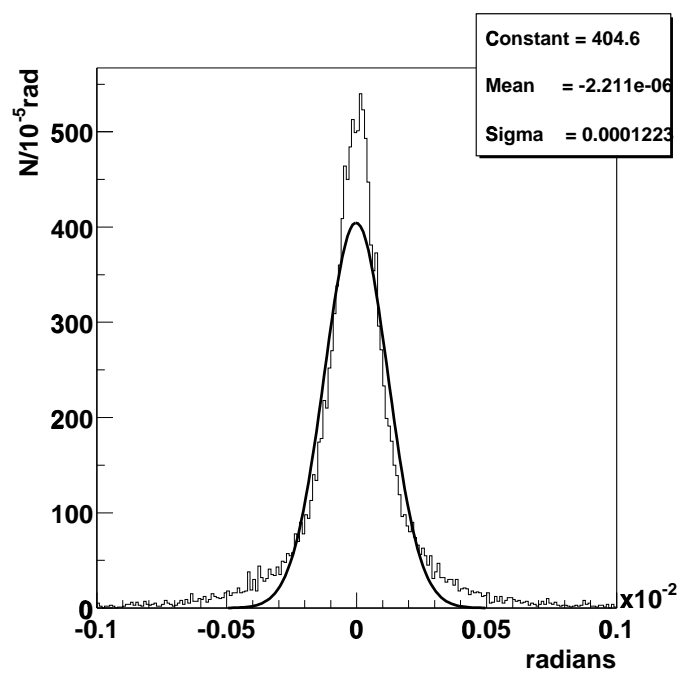

(a)

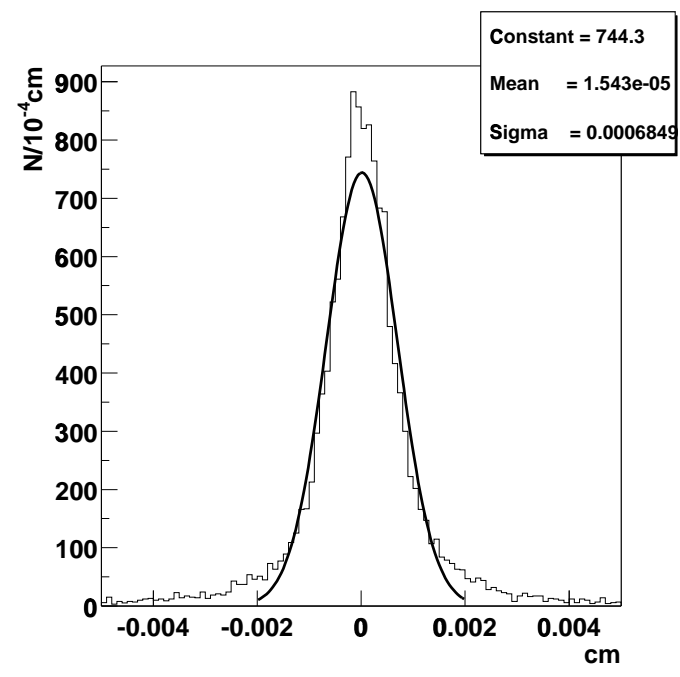

(b)

Figure 4.5: Spatial resolution for the barrels: (a) difference in the angle $\phi$ between reconstructed and MC clusters, (b) resolution in $x_{\text {local }}\left(\Delta\left(x_{\text {local }}^{\text {eco }}-x_{\text {local }}^{M C}\right)\right)$. The fitted function is a Gaussian.

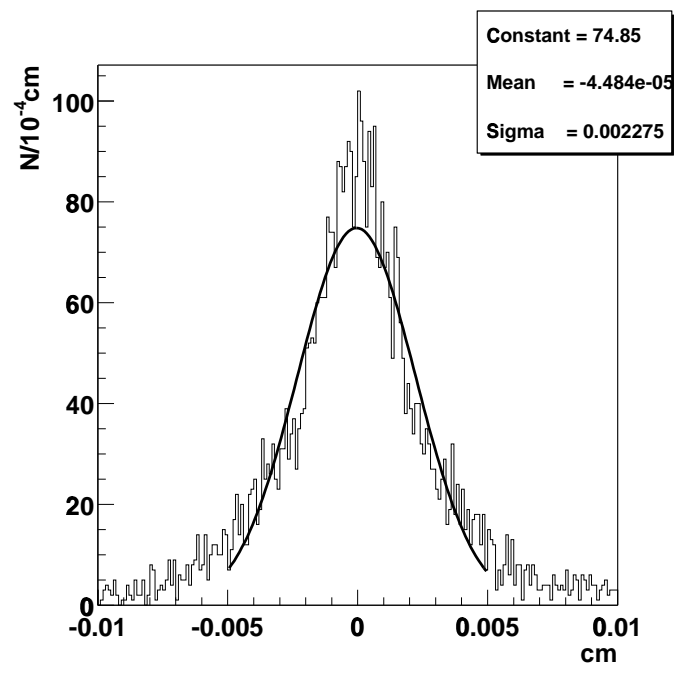

(a)

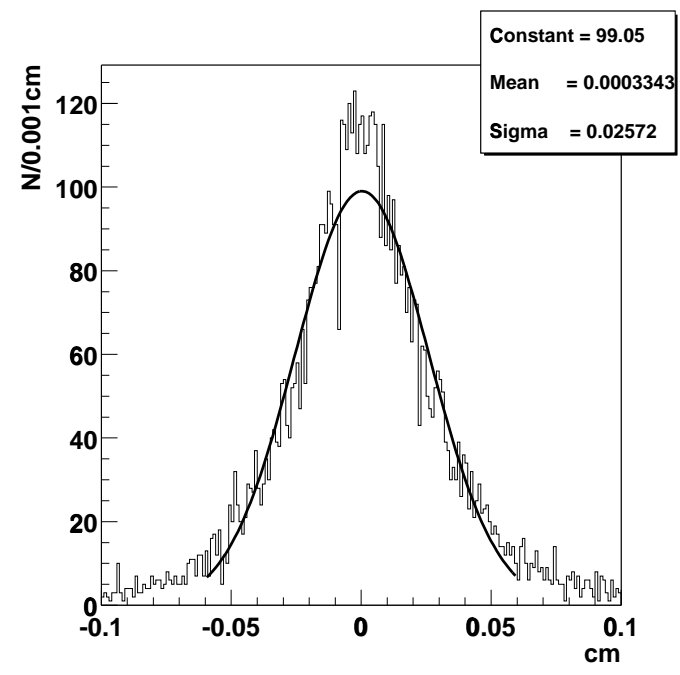

(b)

Figure 4.6: Spatial resolution for the barrels: (a) resolution in $z$ (plotted is the difference between the reconstructed $z$-position and the $\mathrm{MC} z$-position) for layers with a $90^{\circ}$ stereo angle, (b) resolution in $z$ in $2^{\circ}$ stereo layers. 


\section{Barrels: Efficiencies and Misidentification}

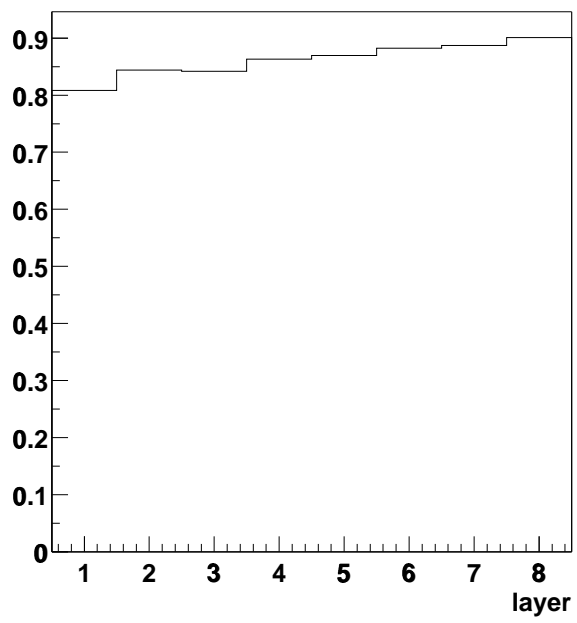

(a)

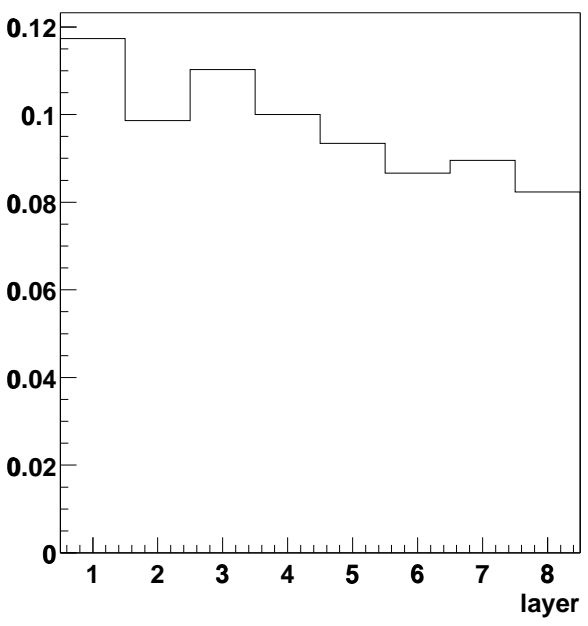

(b)

Figure 4.7: Efficiency (a) and misidentification (b) for 2d clusters on axial layers. A cluster was defined as matched if the distance between the reconstructed and the MC clusters was less than $25 \mu \mathrm{m}$.

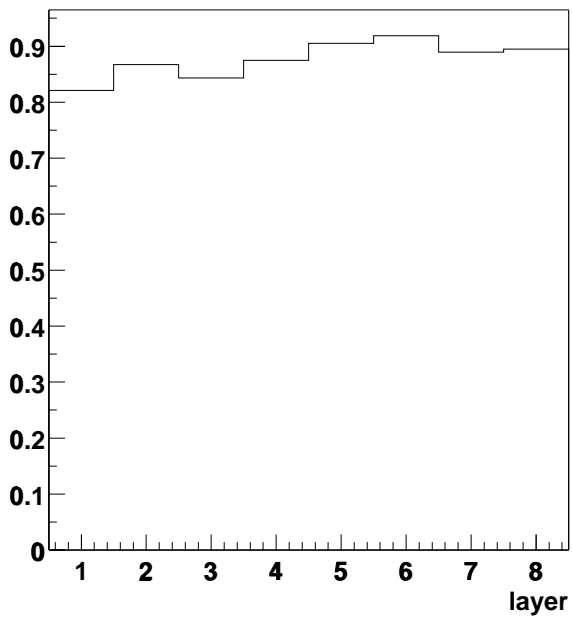

(a)

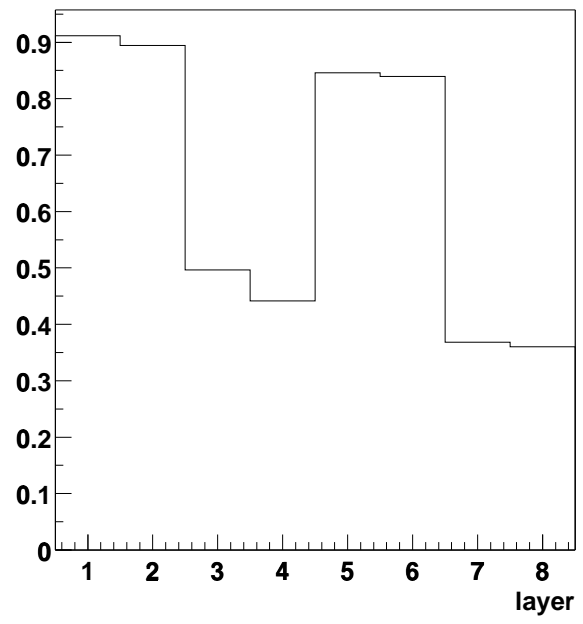

(b)

Figure 4.8: Efficiency (a) and misidentification (b) for 3d clusters. A cluster was defined as matched if the distance between the reconstructed and the MC cluster was less than $150 \mu \mathrm{m}$ for clusters on $90^{\circ}$ layers and $1000 \mu \mathrm{m}$ for clusters on $2^{\circ}$ layers. The extremely high misidentification in the $90^{\circ}$ layers is due to multiplexes (and their ghosts) on top of ghosts and genuinely wrong clusters. 


\section{Disks: Resolutions}

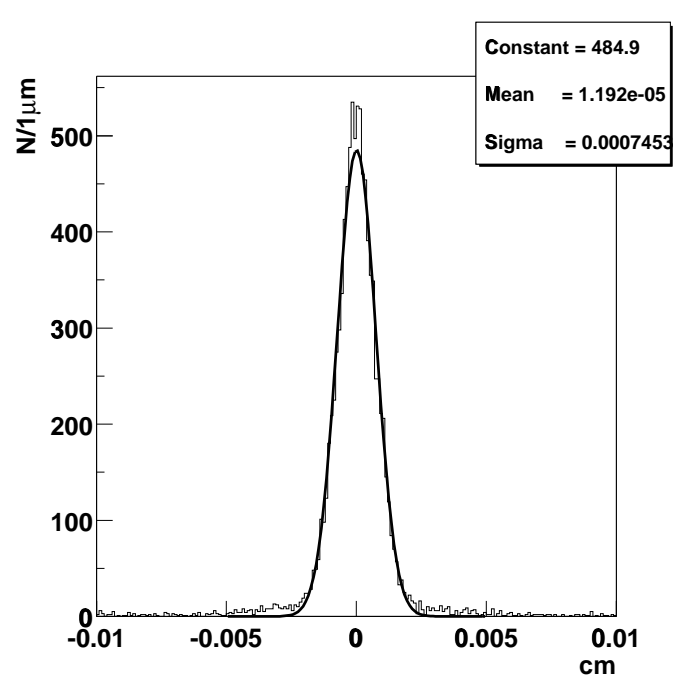

(a) resolution in $x_{\text {local }}$

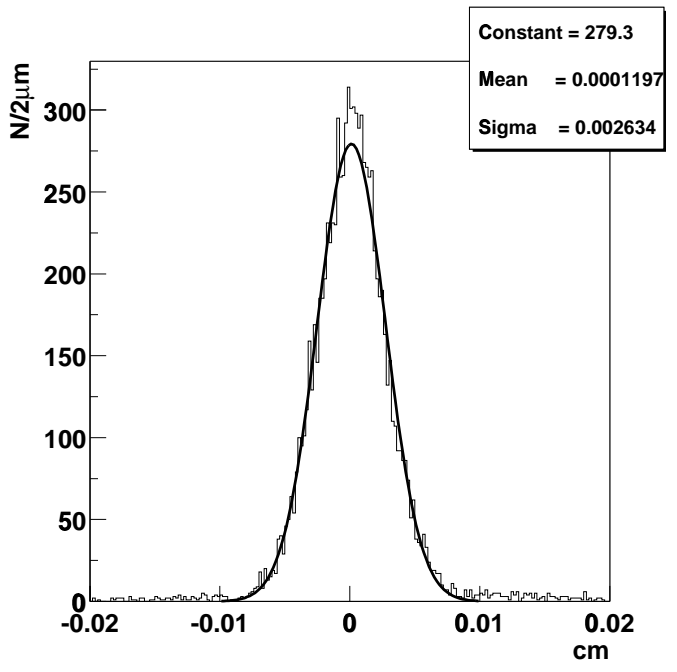

(b) resolution in $z_{\text {local }}$

Figure 4.9: Spatial resolution for F-Disks. Plotted is the difference between the Monte Carlo and the reconstructed cluster position for each reconstructed cluster. For the definiton of $x_{\text {local }}$ and $z_{\text {local }}$ see fig. 4.1.

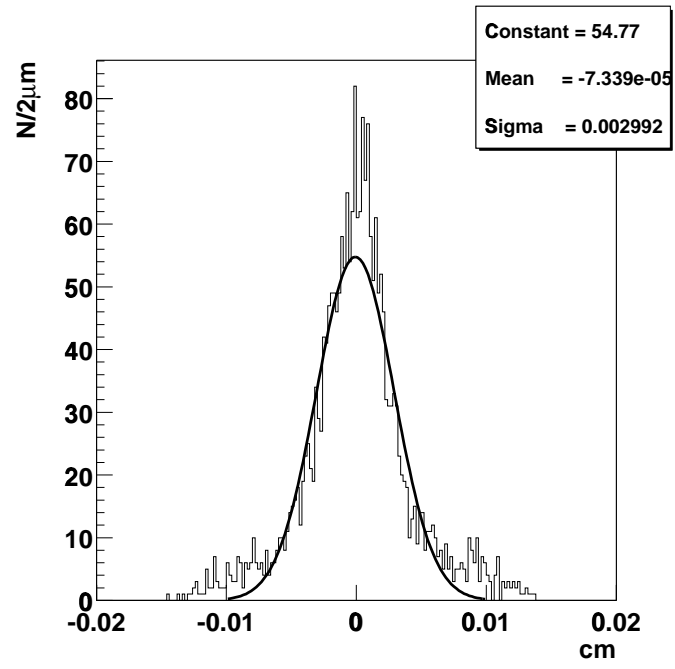

(a) resolution in $x_{\text {local }}$

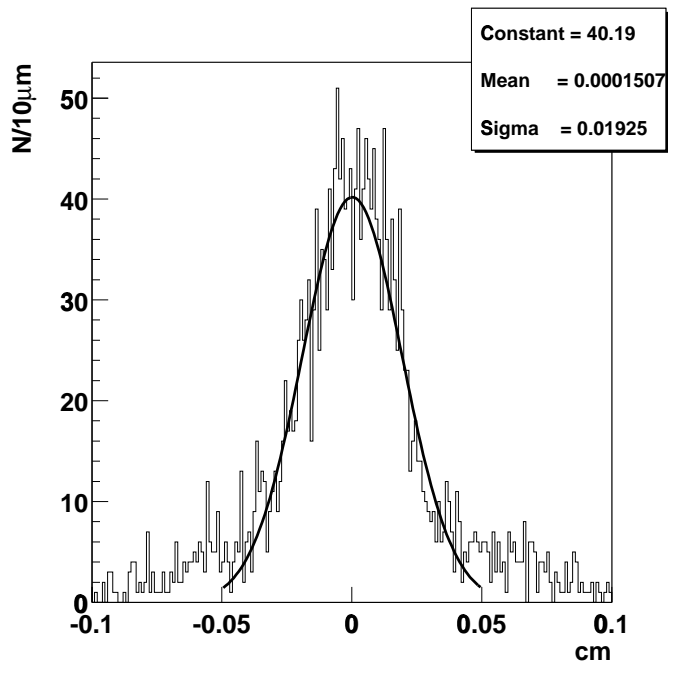

(b) resolution in $z_{\text {local }}$

Figure 4.10: Spatial resolution for H-Disks. Plotted is the difference between the Monte Carlo and the reconstructed cluster position for each reconstructed cluster. 


\begin{tabular}{|l|c|}
\hline Coordinate & Resolution \\
\hline Barrels: $x_{\text {local }}$ & $6.8 \mu \mathrm{m}$ \\
Barrels $\left(2^{\circ}\right): z_{\text {global }}$ & $257 \mu \mathrm{m}$ \\
Barrels $\left(90^{\circ}\right): z_{\text {global }}$ & $23 \mu \mathrm{m}$ \\
F-Disks: $x_{\text {local }}$ & $7.5 \mu \mathrm{m}$ \\
F-Disks: $z_{\text {local }}$ & $26 \mu \mathrm{m}$ \\
H-Disks: $x_{\text {local }}$ & $30 \mu \mathrm{m}$ \\
H-Disks: $z_{\text {local }}$ & $192 \mu \mathrm{m}$ \\
\hline
\end{tabular}

Table 4.1: Summary of SMT cluster resolutions for Level 3.

\section{Timing}

As can be seen in table 3.3, there are severe time constraints on all the trigger components. Figs. 4.11(a,b) show the time required for the clustering of $Z \rightarrow \mu \mu$ events with different numbers of minimum bias events added. These plots show that with a few exceptions and even under difficult circumstances (events containing six minimum bias events are rare) the amount of time needed for the clustering is reasonable.

\subsection{Calibration}

The signal measured in each channel in the SMT consists of a pedestal and a gain. The pedestal is the ADC count measured even if there is no signal. The gain is the amount by which the signal increases when a channel registers a hit. Ideally the gain is proportional to the deposited energy. In real data, unlike simulation, the pedestals and gain vary for each channel. Due to time and memory constraints at Level 3 it is not possible to calibrate each channel individually. Instead the pedestals for each chip (cf. section 3.2.2) can be parametrized as a function of the strip number. 


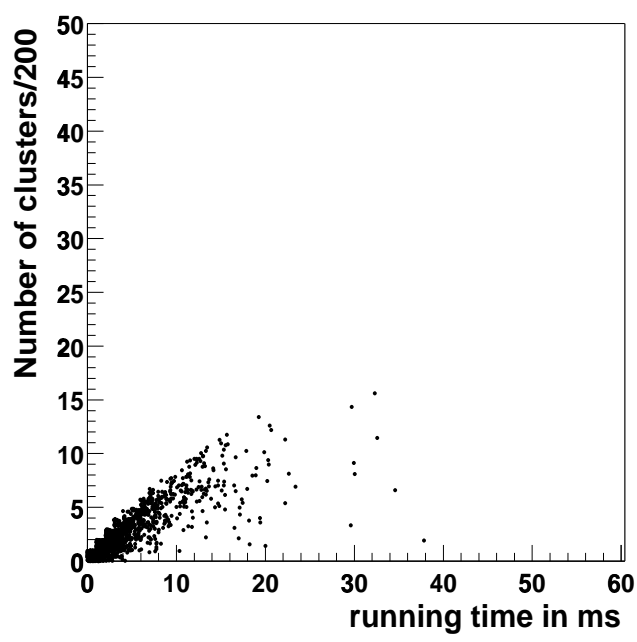

(a) Timing results for $Z \rightarrow \mu \mu$ events without any minimum bias events added.

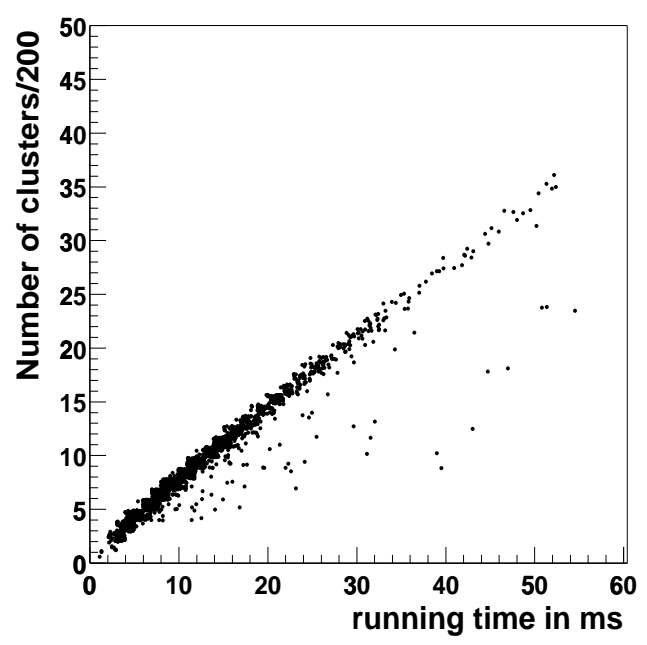

(b) Timing results for $Z \rightarrow \mu \mu$ events with six minimum bias events added.

Figure 4.11: The histograms show the number of clusters versus the time (in milliseconds) needed for their reconstrution. The timing studies were done on a machine comparable to the machines used in the trigger system. The total time allocated for the processing of one event is around 100 milliseconds.

Principally there are two ways of recovering the pedestals:

- From primary data acquisition (PDAQ): The pedestal is the average value in a channel from data taken during ordinary runs.

- From secondary data acquisition (SDAQ): A charge is put on every channel to measure the pedestals without signal. The calibration will use pedestals from SDAQ.

\section{Parametrizing Pedestals}

In the majority of chips the pedestal distribution is flat. It is not fully understood where the non-flat distributions (e.g. fig. 4.12) come from. A probable cause is that the power for the chip electronics (preamplifier etc.) comes from one side of the chip and therefore the voltage applied to the channels is not constant over the range of a chip [55]. 


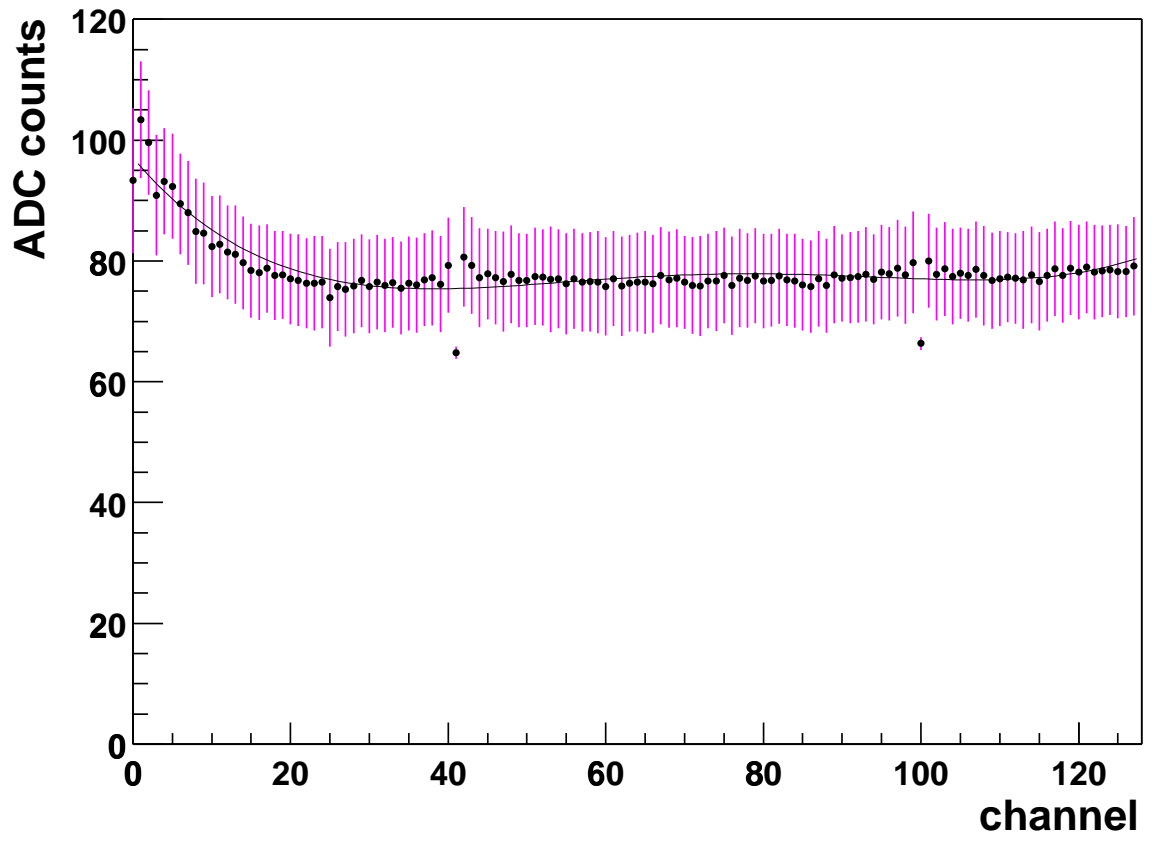

Figure 4.12: Characteristic curve for a chip at the end of a ladder. The errors are from SDAQ measurements and are highly correlated. Dead channels are characterised by an offset and extremely small errors.

The biggest source of error when fitting are dead channels. These channels have an almost constant value and a very low error and therefore a strong influence on the fit (see fig. 4.13(a)). Removing those channels from the fit improves it considerably as shown in fig. 4.13(b). In order to remove the dead channels, initially the channels with the 10 smallest errors were removed from the fit. The influence of removing a low number of working channels during this procedure is negligible. More recent versions of the SDAQ flag individual channels as dead, when necessary, which makes their removal straightforward. The function used to parametrize the pedestals is a fourth order polynomial. This parametrizes the existing data sufficiently well, i.e. the fitted curve almost always lies within the error for each channel (fig. 4.14), but also leaves enough flexibility should the shape of the pedestal curve change over time.

The parametrized pedestals have been tested on real data, but the results have been inconclusive. 


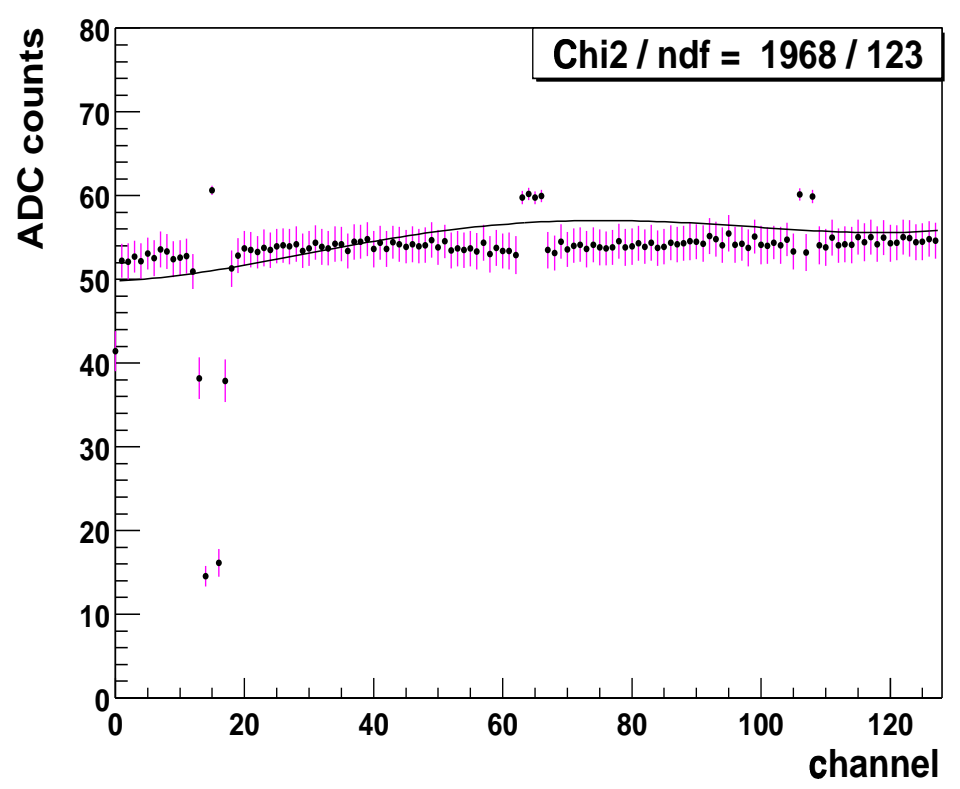

(a)

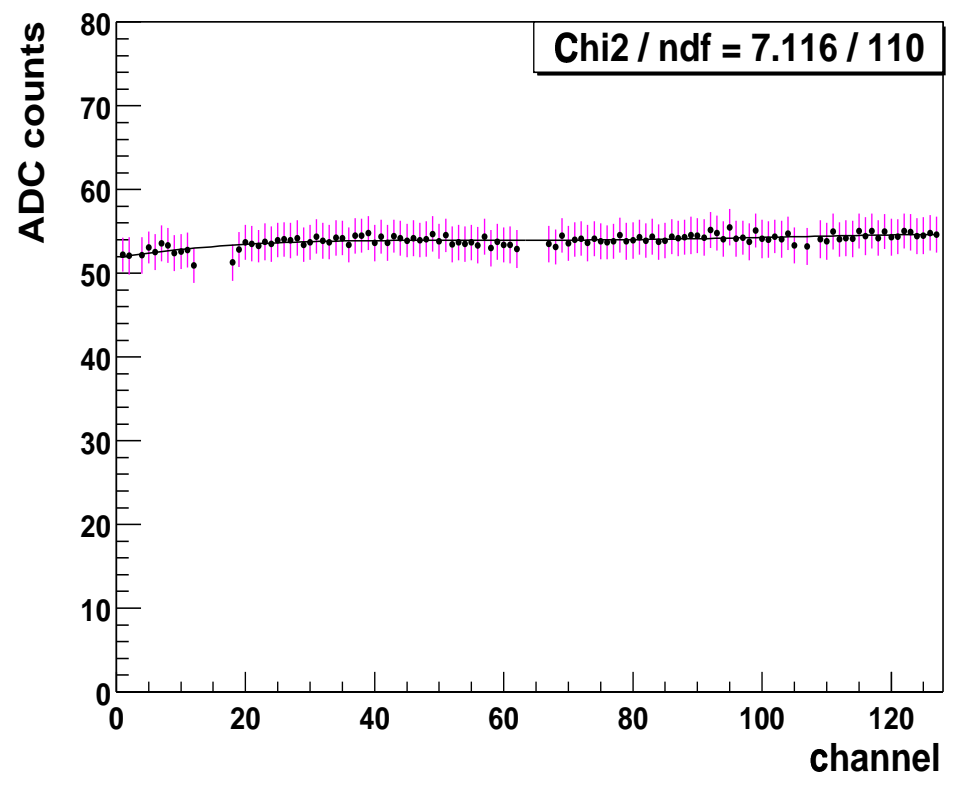

(b)

Figure 4.13: Fig. (a) shows a fit to an otherwise flat pedestal curve including the dead channels on the chip. Fig. (b) shows a considerably improved fit on the same chip after the removal of the dead channels. 


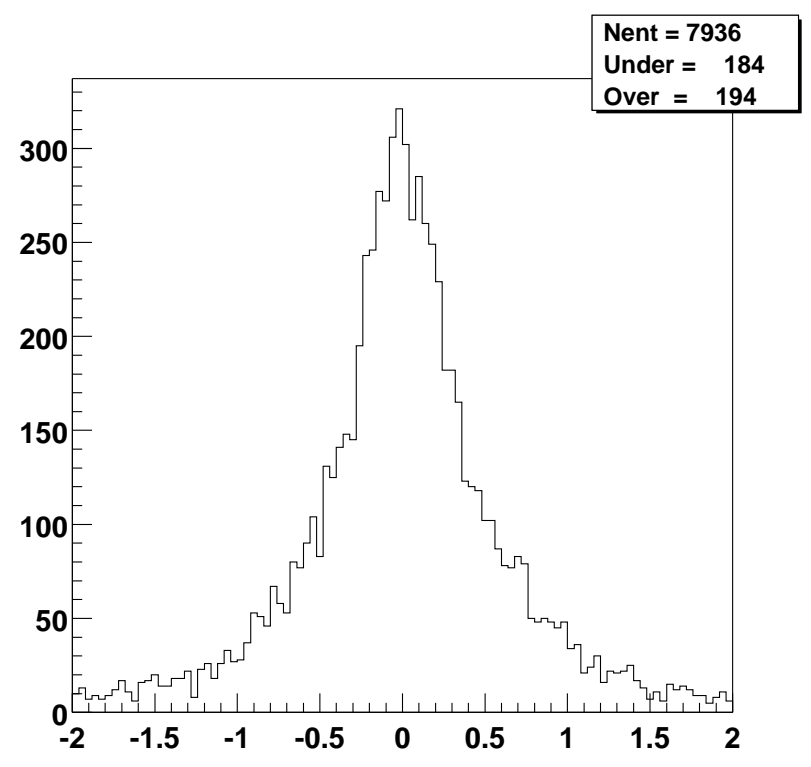

Figure 4.14: Difference between fitted and measure pedestal divided by the error (from calibration) on the measured pedestal. The calibration file contained 62 chips. For the vast majority of channels the difference between the fitted value and the measured value is less than the error on the measurement (i.e the absolute of this quantity is less than 1 ).

\subsection{Cosmics}

One of the SMT barrels was used to collect cosmic data before integrating it into the detector. A schematic [56] of the test stand is given in fig. 4.15. The scintillators were used for triggering and the iron plate for momentum cuts. An event was accepted by the following trigger decisions:

$$
(A \text { and } B \text { ) and } C \text { or } \quad C \text { and } D
$$

Not all ladders of this barrel were read out. These ladders appear as light grey in the figures. For readout, the actual collider data readout system was used.

As a proof of principle, 500 events $^{4}$ of this test run were used to reconstruct tracks

\footnotetext{
${ }^{4}$ The number of events was limited by the difficulties in calibrating the detector. The calibration file for these events was provided by R. Illingworth.
} 


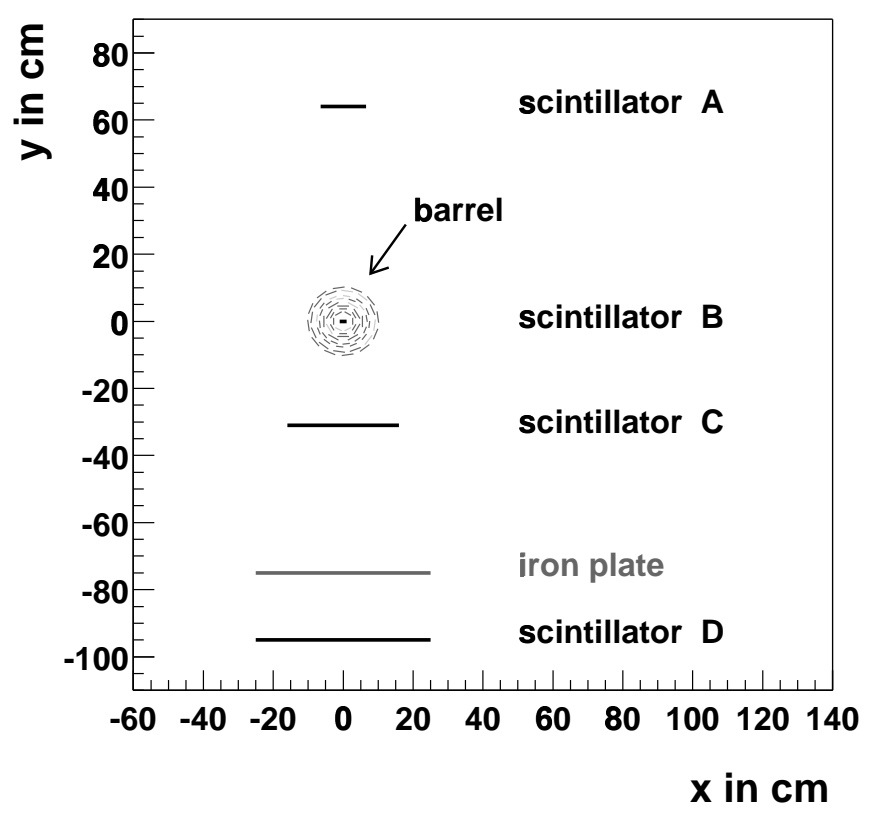

Figure 4.15: Setup for the cosmic ray test (to scale).

originating from cosmics using the Level 3 clustering and a specialized cosmic track finder.

\section{A Cosmic Track Finder}

Unlike tracks reconstructed during collisions, the tracks caused by cosmics in the test-setup were straight as no magnetic field was present and they did not originate close to $x=y=0$. The tracking algorithm used for cosmic tracks is as follows:

- Only clusters where information in $x, y$ and $z$ is available are used.

- Each hit is combined with every other hit, except when both are on the same ladder. These combinations can be represented by a point in $\left(\tan (\lambda), y_{0}\right)$-space where $\tan (\lambda)$ is the angle relative to the $y$-axis and $y_{0}$ the point at which the track candidate would cross $y=0$ (see fig. 4.16).

- Cuts on $\tan (\lambda)$ and $y_{0}$ ensure that track candidates lie within the detector range and are consistent with cosmics (i.e. not parallel to the $x$-axis). 


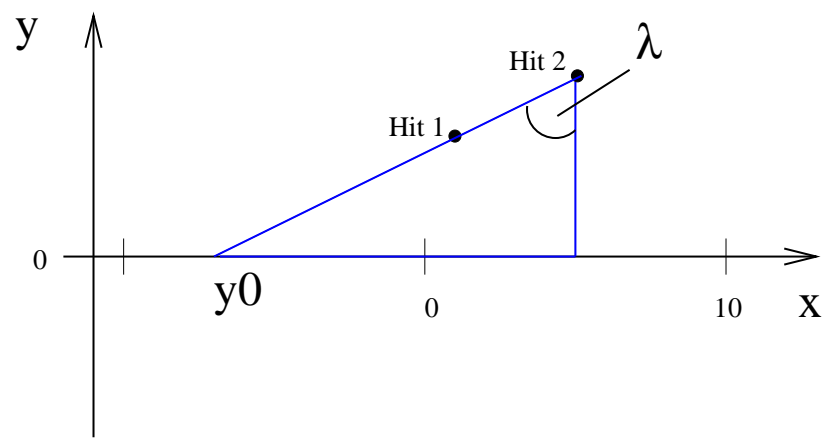

Figure 4.16: Variables used by the cosmic track finder.

- A track candidate is found by finding the biggest 'cluster' of points in $\left(\tan (\lambda), y_{0}\right)$ space.

- Minimum requirement is 4 hits on at least 4 different layers.

- A straight line is fitted (cf. Appendix A.4) to all points belonging to this 'cluster'.

- Once an $(x, y)$ track is fitted an attempt is made to find a straight line in $(z, y)$ using all $3 \mathrm{~d}$-clusters associated with the axial clusters used in the previous fit.

Using this algorithm 187 tracks in 500 events are found. The offline reconstruction finds 210 tracks in the same sample [57]. This is due to better calibration, which results in slightly more clusters and less noise. In events where both online ${ }^{5}$ and offline reconstruction find a track, they find the same track. Figs. 4.17 and 4.18 show the same (typical) event, with a reconstructed track, from online and offline reconstruction respectively.

Fig. 4.19 shows the track residuals in $x$ and fig. 4.20 the track residuals in $z$ for online reconstruction. For comparison the track residuals in $z$ for offline reconstruc$\operatorname{tion}^{6}$ are given in fig. 4.21. They are in reasonable agreement with the online results.

\footnotetext{
${ }^{5}$ Any reconstruction using trigger software is referred to as 'online'.

${ }^{6}$ Track residuals in $x$ were not available for offline reconstruction.
} 


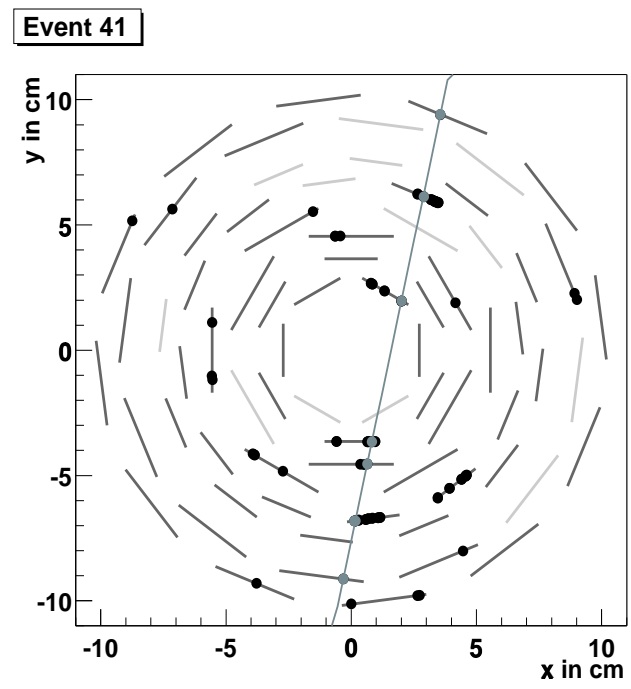

(a)

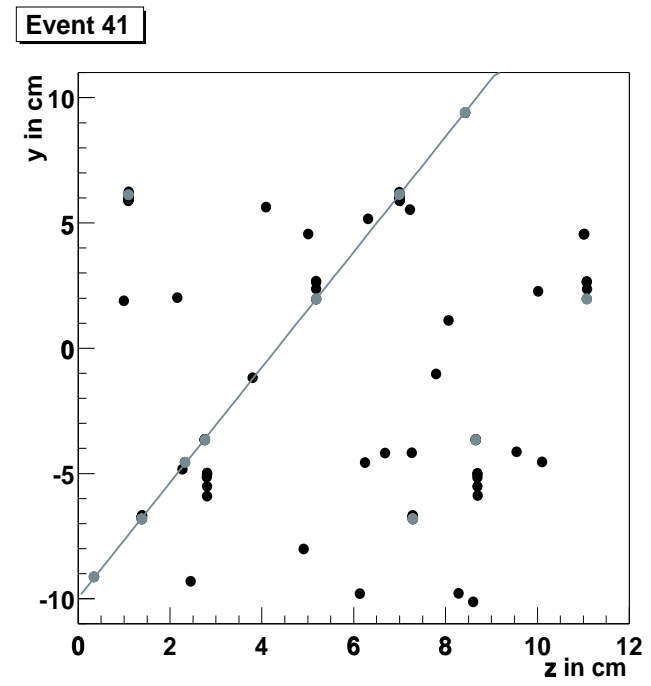

(b)

Figure 4.17: Event display for a cosmic track. Ladders which are not read out appear light grey. In fig. (b) the lighter clusters are all possible $z$ values belonging to the clusters used for the trackfit in $x-y$.

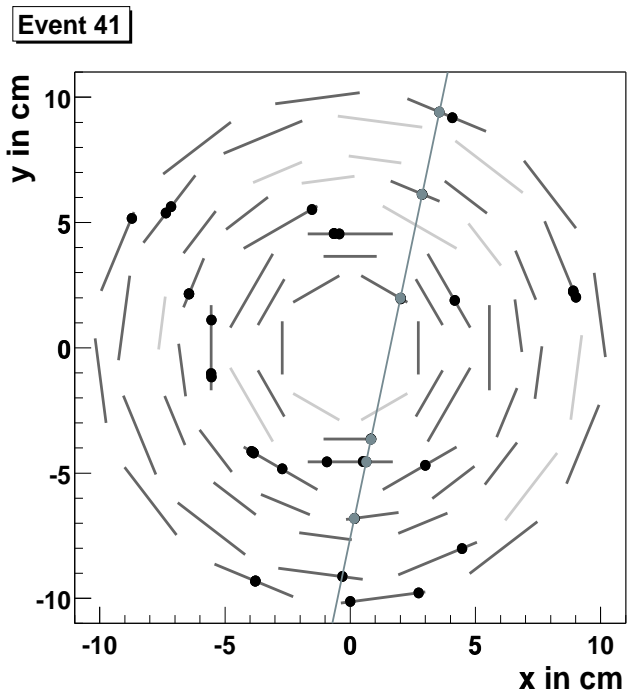

(a)

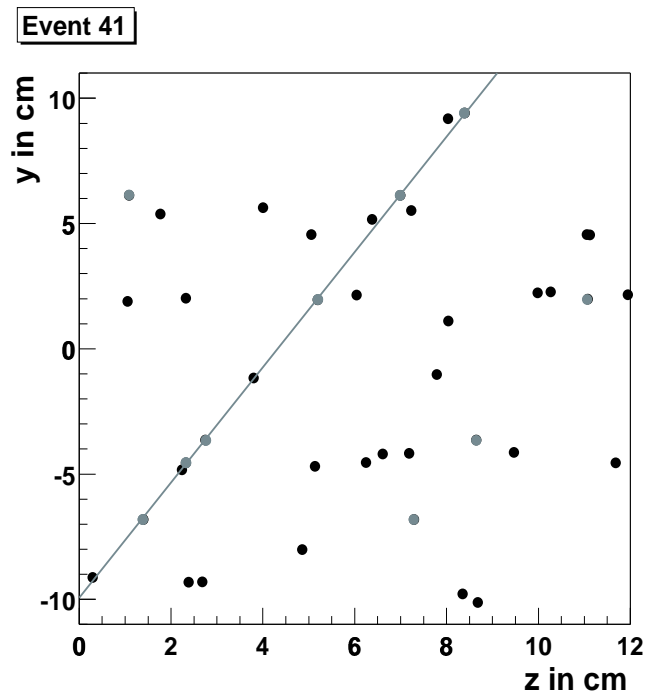

(b)

Figure 4.18: The same event as in fig. 4.17 from offline reconstruction [58]. 


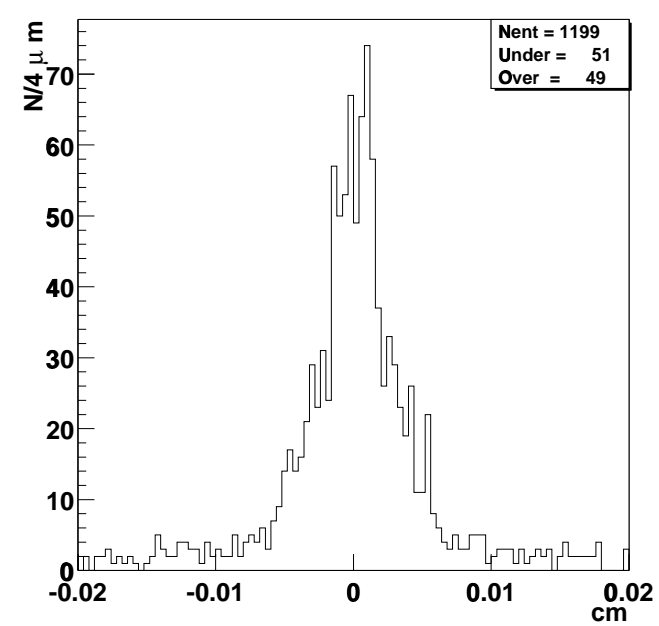

Figure 4.19: Track residuals in $x\left(\Delta\left(x_{\text {track }}-x_{\text {cluster }}\right)\right)$.

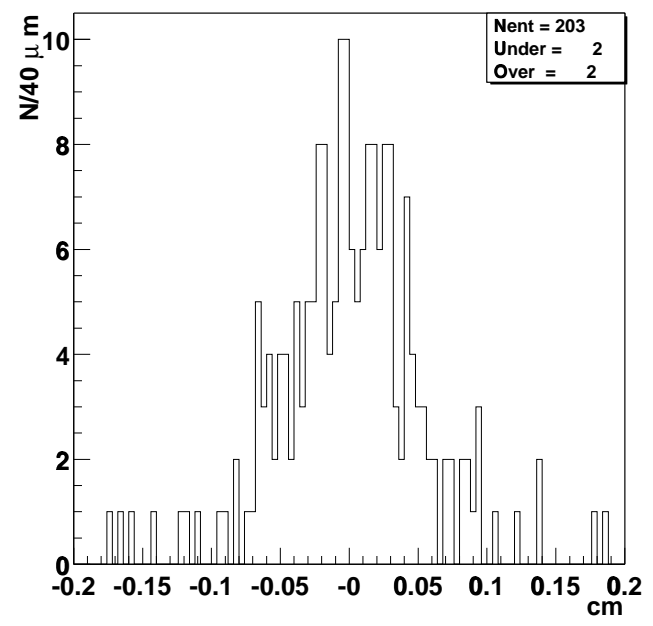

(a) $2^{\circ}$ ladders.

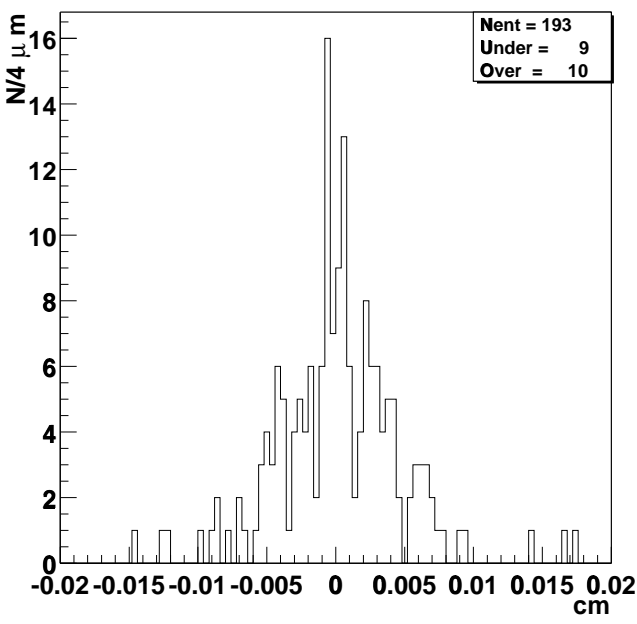

(b) $90^{\circ}$ ladders.

Figure 4.20: Track residuals in $z\left(\Delta\left(z_{\text {track }}-z_{\text {cluster }}\right)\right)$ for tracks made exclusively of $2^{\circ}$ or $90^{\circ}$ clusters. 

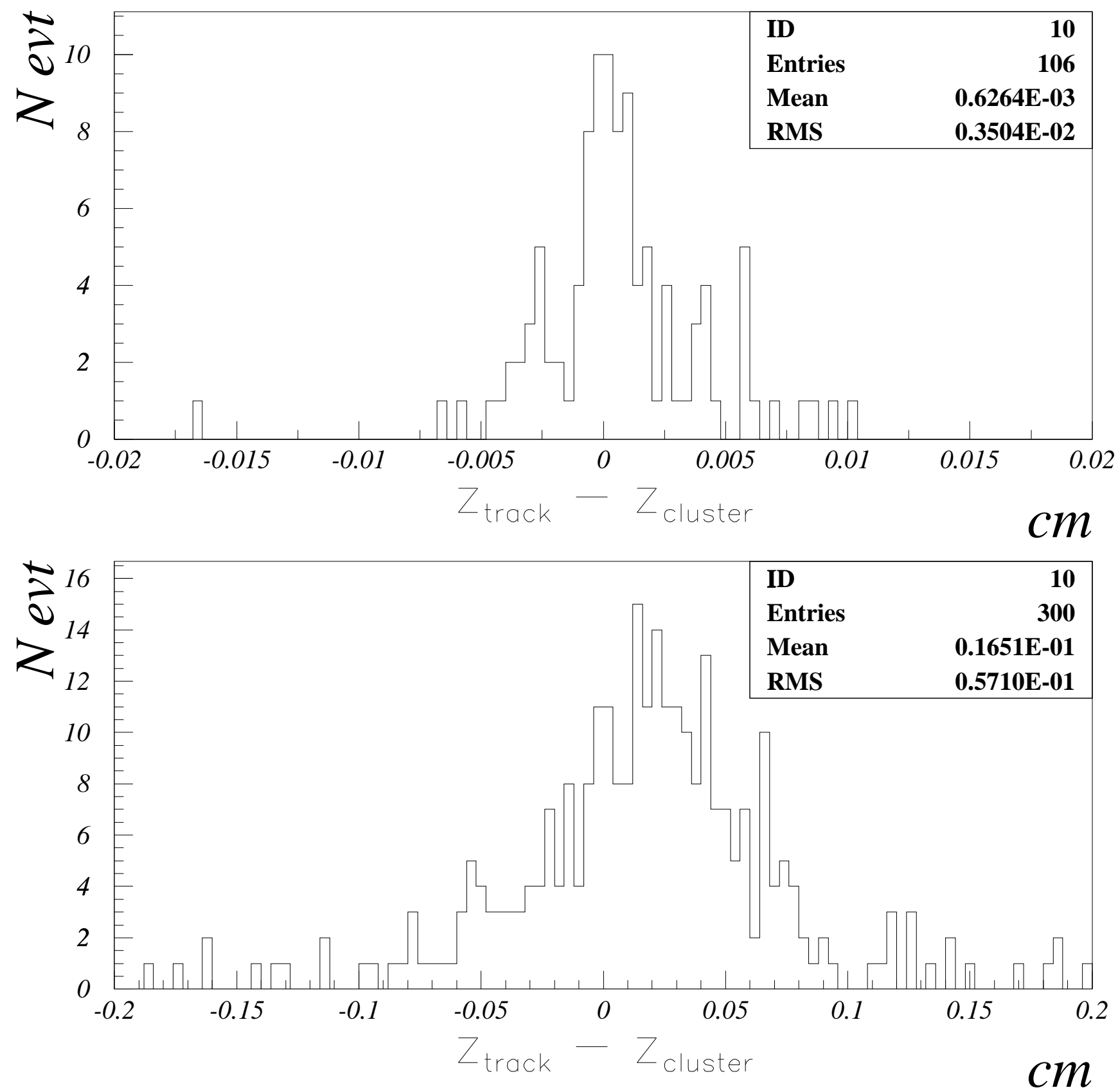

Figure 4.21: Resolution in $z$ (top: $90^{\circ}$, bottom: $2^{\circ}$ ) from offline reconstruction [57]. 


\section{Chapter 5}

\section{Analysis}

\section{$5.1 \quad$ Introduction}

In this chapter Monte Carlo studies for lifetime measurements in the decays $B_{s}^{0} \rightarrow J / \psi \phi$ and $B_{d}^{0} \rightarrow J / \psi K^{* 0}$ are presented. A greater emphasis is given to $B_{s}^{0} \rightarrow J / \psi \phi$ as $B_{s}^{0}$ mesons are currently exclusive to the Tevatron. While both decays ${ }^{1}$ have been measured at the Tevatron before, Run II presents the first opportunity to attempt these measurements at $\mathrm{D} \varnothing$. The initial measurements will provide a valuable check for the reconstruction software by comparing the results to previous measurements (cf. table 2.1, p. 28). Later in the run, once the detector is fully understood, more precise measurements will help to validate predictions made by HQET as mentioned in section 2.6 .

As outlined in chapter 2, lifetime measurements form the basis for other measurements, e.g. $\frac{\Delta \Gamma}{\Gamma}$. Investigating those require more sophisticated Monte Carlo generators than the one available at the time of writing. Section 5.7 outlines the problems encountered and describes the implementation of a new generator which will make these simulations possible in the future.

At the end of the chapter the results of my search for $J / \psi$ mesons in the first consistent set of real data that became available in November 2001 are presented.

\footnotetext{
${ }^{1}$ References to a decay in this chapter always refer to the charge-conjugated state as well.
} 


\subsection{The Monte Carlo Data Sample}

\subsubsection{Software}

Within the $\mathrm{D} \emptyset$-framework there are four stages in the production of a Monte Carlo data sample. PYTHIA [59] is used to simulate the creation of the $B$-mesons and the underlying event. It also handles the decays of all particles except the $B$-mesons. These are decayed by QQ [60], the CLEO event generator. The results are written out as so-called McKineChunks, which store the four-vector and vertex information for each particle along with links to its parent and/or daughters. The cuts in PYTHIA are kept to a minimum to avoid distorting the momentum distributions of the $B$-mesons. But to prevent excessive CPU consumption, only events which contain the signal channel (e.g. $B_{s}^{0} \rightarrow J / \psi \phi$ ) and pass certain cuts, e.g. a minimum transverse momentum for the decay products, are selected from the PYTHIA/QQ output for further processing.

For these events the detector response is simulated using the d0gstar (D $\varnothing$ GEANT Simulation of the Total Apparatus Response) package. The data are then digitized and minimum bias events are added to the signal by the d0sim package. Output is in the raw data format. These files can then be processed by the reconstruction software, d0reco. Most software components include an analysis package. The results of these analyses are written into an ntuple. While using the content of this ntuple cannot replace a full analysis, it is a good starting point to check the quality of the data and perform basic particle reconstruction ${ }^{2}$. The $J / \psi$ reconstruction in real data in this chapter is based on an ntuple-analysis using the ROOT [61] software package.

All software components at $\mathrm{D} \varnothing$ are still under heavy development. Once every week all updates to the software are combined and released to the collaboration. These software releases are huge and only a limited number of them is available at a time. To facilitate the production and analysis of data (as opposed to software development) certain releases are designated 'production releases'. These releases are

\footnotetext{
${ }^{2}$ ROOT tuple analyses are an 'approved' DØ practice.
} 
supposed to be stable and only bug-fixes to the code should have been allowed. In practice this did not always work and parts of the software would regularly display unexpected $^{3}$ behaviour.

\subsubsection{Producing a Monte Carlo signal sample}

$\mathrm{D} \emptyset$ uses computer farms around the world to produce large amounts of Monte Carlo data. Most of these samples are of a general nature (e.g. QCD) and not really useful for a more specialized analysis like $B_{s}^{0} \rightarrow J / \psi \phi$. Producing Monte Carlo data for exclusive decay channels is slightly more complicated and so data in the $b$-physics group was initially produced by individuals using local machines on request. This proved to be very inefficient. I was involved in the efforts of the $b$-physics group to use the computer farms instead. The fact that most of the $B$-meson decays need a selection step as described earlier after the initial generation of data prevented a full automation of the process. Nevertheless the first files were reconstructed on the farms in November 2001. A close investigation of these files revealed a number of problems, so the data sets used in this analysis have been re-made several times. My work in this area lead to the decision to change generators after I showed that the EvtGen generator [62] was better at modelling $b$-decays than the current default generator QQ. (cf. section 5.7.1). A decision was made not to use the farms for large scale production until the group was confident that the problems with the generators were fully understood [63].

The 7000 signal events for each of the decay channels represent a compromise between the difficulty of producing Monte Carlo data and the need to produce results. It should be emphasized that the aforementioned problems in producing Monte Carlo data are purely software related and will be resolved in the future.

\footnotetext{
${ }^{3}$ Like the magnetic field changing direction.
} 


\subsubsection{Background sample}

The exact backgrounds depend heavily on the triggers used. A trigger simulation is being developed to estimate these backgrounds, but was not available for this study. The main background for $B$-meson reconstruction in both signal channels is expected to come from events containing real $J / \psi$ which are combined with random tracks to form fake $B$-mesons [11]. The ratio [71] of direct (i.e. produced during the hard-scatter process) $J / \psi$ and $J / \psi$ mesons coming from $b$-decays is expected to be approximately 80 : 20. During Run I CDF found that zero-lifetime background was the main background component in the $B_{s}^{0} \rightarrow J / \psi \phi$ lifetime measurement, with an overall signal to background ratio [64],[71] of $\sim 0.9$. This background can be considerably reduced when measuring the angular distributions in these decays by requiring a minimum lifetime for the reconstructed $B$-mesons. Due to their generally low transverse momentum muons from $\pi$ and $K$ decays are not expected to pollute considerably the $J / \psi$ signal. Most fake $J / \psi$ are a combination of a muon produced in the hard-scatter process and a muon from a $\pi$ or $K$ decay. Preliminary results [65] from Run II indicate that this background will be small. The background resulting from reconstructing a $B_{d}^{0}$ decaying to $J / \psi K^{* 0}$ as $B_{s}^{0} \rightarrow J / \psi \phi$ or vice-versa is often referred to as cross-talk and is discussed further in section 5.5. The branching ratios of 'non-resonant' decays (e.g. $B_{d}^{0} \rightarrow K^{* 0} \mu^{+} \mu^{-}$) which result in the same final state as the signal channels are extremely small $\left(\sim 10^{-7}\right.$ for the full decay chain $)$ and are not expected to pose a problem.

Even though a proper simulation of the backgrounds was not possible at this time, as a preliminary measure all available events ${ }^{4}$ containing $J / \psi \rightarrow \mu \mu$ were used as background. These samples consisted of $24750 q \bar{q} \rightarrow J / \psi(\rightarrow \mu \mu) X$ and 24750 $b \bar{b} \rightarrow J / \psi(\rightarrow \mu \mu) X$ events. The second sample contained a number of signal events, 879 for $B_{s}^{0} \rightarrow J / \psi \phi$ and 4056 for $B_{d}^{0} \rightarrow J / \psi K^{* 0}$. As a precautionary measure these events were not used, since there is currently no mechanism to retrieve the exact parameters (e.g. momentum cuts, decay tables, etc.) used to generate a particular

\footnotetext{
${ }^{4}$ Courtesy of the New Phenomena group.
} 
file $^{5}$. However in order to determine the expected event yields (see section 5.6) a knowledge of all generator level cuts is necessary. Both signal samples were also used as background for each other.

\section{Monte Carlo particle masses}

All particles were simulated with masses very close or identical to their values as listed in the PDG [3]. These masses are throughout the text referred to as $m_{\text {particle }}^{\mathrm{PDG}}$. They are listed in table 5.1.

\begin{tabular}{|l|c|}
\hline$J / \psi$ & $3096.87 \pm 0.04 \mathrm{MeV}$ \\
$\phi$ & $1019.417 \pm 0.014 \mathrm{MeV}$ \\
$K^{* 0}$ & $896.10 \pm 0.27 \mathrm{MeV}$ \\
$B_{d}^{0}$ & $5279.4 \pm 0.5 \mathrm{MeV}$ \\
$B_{s}^{0}$ & $5369.6 \pm 2.4 \mathrm{MeV}$ \\
\hline
\end{tabular}

Table 5.1: Particle masses according to the Particle Data Group(PDG) [3].

\footnotetext{
${ }^{5}$ This is a known problem at $\mathrm{D} \emptyset$.
} 


\section{$5.3 \quad \mathrm{~B}_{\mathrm{s}}^{0} \rightarrow \mathrm{J} / \psi\left(\rightarrow \boldsymbol{\mu}^{+} \boldsymbol{\mu}^{-}\right) \phi\left(\rightarrow K^{+} K^{-}\right)$}

\subsubsection{Muon reconstruction}

In order to reach the muon chambers a particle needs a minimum $p_{t}$ of $1.5 \mathrm{GeV}$. To reduce the amount of principally unreconstructable data in the MC sample a cut at generator level ensured that muons coming from the $J / \psi$ had a minimum $p_{t}$ of $1.5 \mathrm{GeV}$. They were also required to have $|\eta|<2$, equivalent to the coverage of the muon system. All other muons were unrestricted. Fig. 5.1 shows the generated muon $p_{t}$ spectrum for the $7000 B_{s}^{0} \rightarrow J / \psi \phi$ signal events.

The muon reconstruction is based on a match between a track reconstructed by the central tracking system (SMT and CFT), here referred to as a 'global track', and a signal in the muon system. Overlaid on the generated $p_{t}$ spectrum in fig. 5.1 are the $p_{t}$ spectra of all muons found in the global tracks and of all those identified by the muon system. It shows clearly that while most muons at low $p_{t}(1.5-2.5 \mathrm{GeV})$ will leave a track in the central tracking system, they often cannot be identified as muons by the muon system.

While the muon system has its own magnetic field and a coarse momentum reconstruction $^{6}$, the momentum of the muon candidates is that of the global tracks they are based on. Fig. 5.2 shows the overall momentum resolution for muons. The momentum resolution depends on the absolute momentum, and will generally worsen at higher momenta. Fig. 5.3 shows the muon $p_{t}$ resolution as a function of $p_{t}$.

The main backgrounds for muons are expected to be cosmic rays, particles other than muons reaching the muon chambers (punch-through) and combinatorics, where combinatorics refer to hits in the muon chambers originating from one track being reconstructed as two or more different tracks. While this background is expected to pose a major problem for the triggers [66], combining information from the central tracking system and the muon chambers will reduce this background considerably in the offline reconstruction. Fig. 5.4 shows the purity as a function of $p_{t}$ for the

\footnotetext{
${ }^{6}$ The expected momentum resolution $\Delta p / p$ in the muon system is $\sim 20 \%$ at threshold, worsening with increasing momentum [67].
} 
$B_{s}^{0} \rightarrow J / \psi \phi$ sample. Except for low $p_{t}\left(p_{t}<2 \mathrm{GeV}\right)$ muons, around $90 \%$ of all muon candidates can be matched to an MC muon.

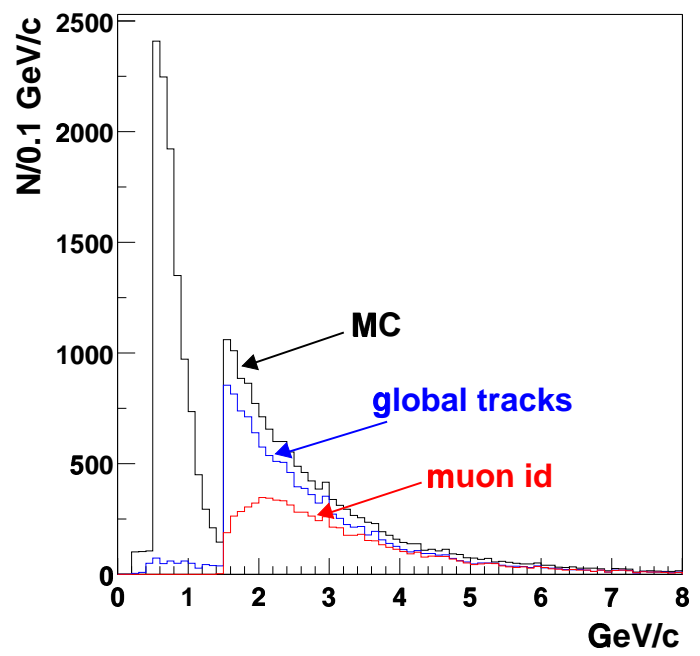

Figure 5.1: Transverse momentum distribution for simulated muons, global tracks matched to $\mathrm{MC}$ muons and muon candidates matched to MC muons. There was a generator level cut requiring the muons in the signal channel to have $p_{t}>1.5 \mathrm{GeV}$. All other muons were unrestricted, resulting in the peak at low $p_{t}$.

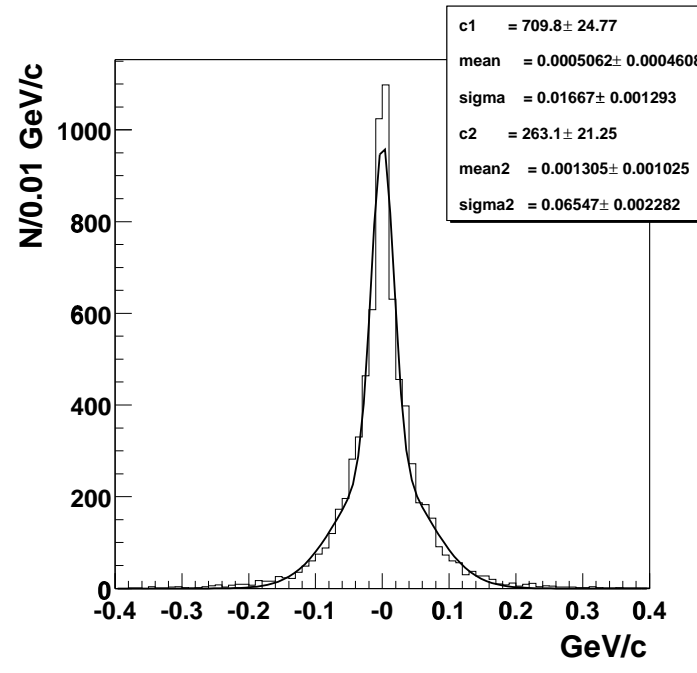

(a) $p_{x}$

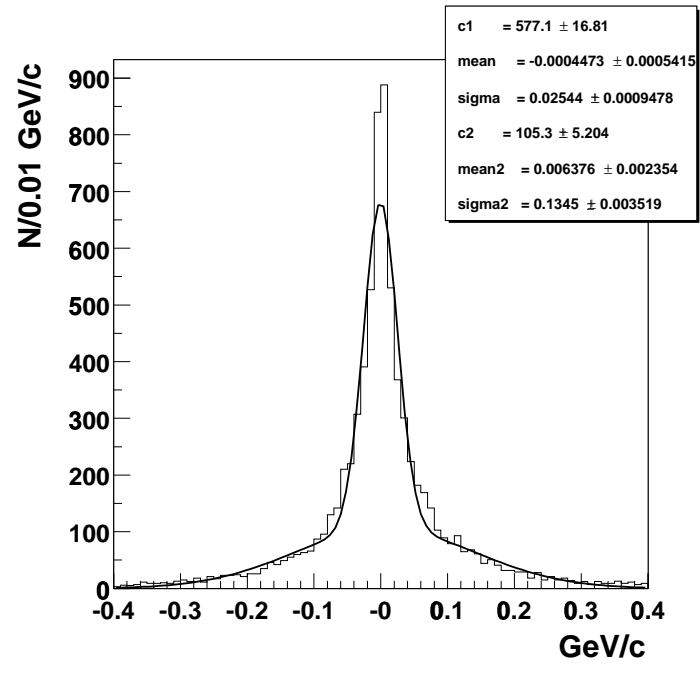

(b) $p_{z}$

Figure 5.2: Muon momentum resolution (MC momentum - reconstructed momentum). The fitted function is a double Gaussian. 


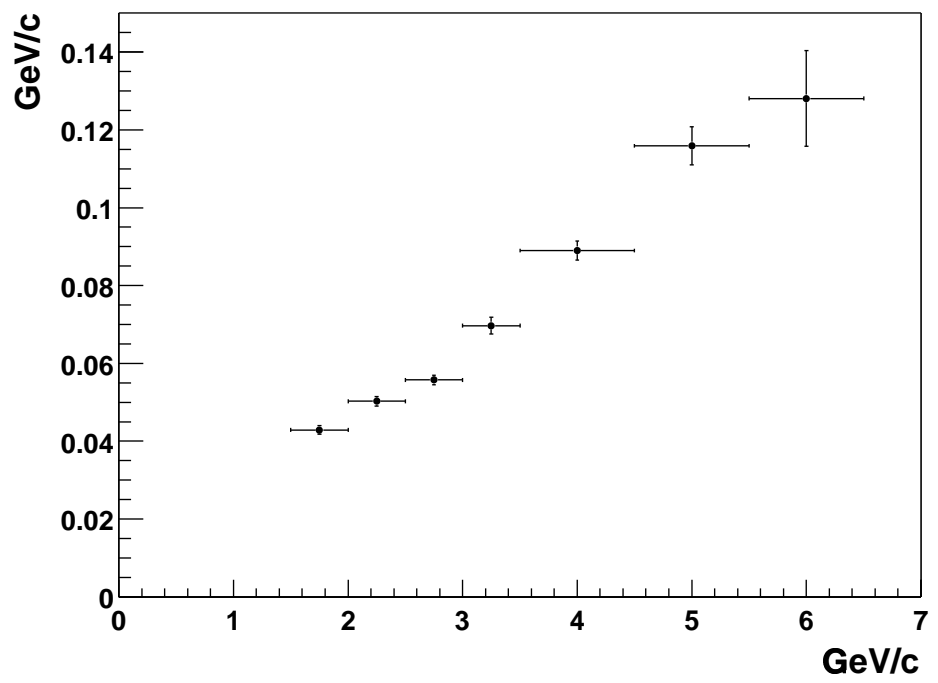

Figure 5.3: Muon $p_{t}$ resolution as a function of $p_{t}$. With increasing $p_{t}$ the tracks become straighter and the resolution worsens. The resolutions for the first four points $(1.75,2.25,2.75,3.25 \mathrm{GeV})$ were derived from all muon candidates with a $p_{t}$ of $\pm 0.25 \mathrm{GeV}$ around the central value. As there were fewer muons available at higher $p_{t}$, the bin size was increased to $\pm 0.5 \mathrm{GeV}$ around the central values of 4.0, 5.0 and $6.0 \mathrm{GeV}$. A Gaussian was fitted to the difference between the reconstructed and the MC $p_{t}$ in all cases. Plotted is the $\sigma$ of each Gaussian and its error vs. $p_{t}$.

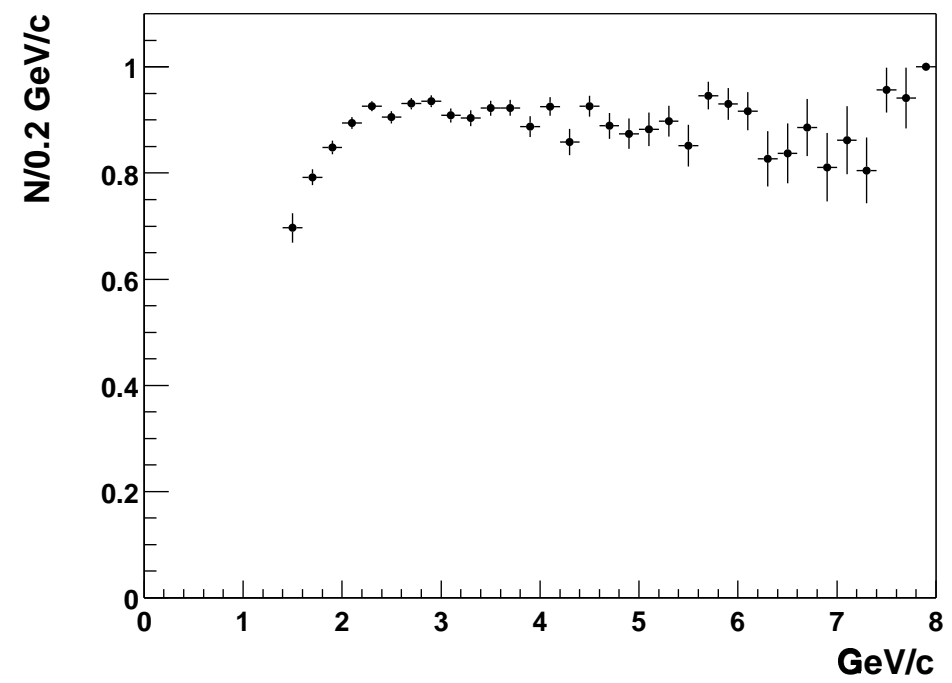

Figure 5.4: Purity: Number of matched muon candidates per $p_{t}$ bin divided by the number of all muon candidates per bin. The Monte Carlo matching was based on the comparison of the generated and reconstructed momenta with a fixed cut-off value ( $\sim 3 \sigma$ of the momentum resolution). The fixed cut-off is justified by the fact that a badly reconstructed muon (like a fake muon) is not useful for this analysis. As the momentum resolution worsens for higher momenta (fig. 5.3), there are a greater number of high $p_{t}$ tracks generated by real muons that remain unmatched, therefore causing a slight drop in purity. 


\subsection{2 $J / \psi$ reconstruction}

$J / \psi$ mesons are reconstructed by combining two oppositely charged tracks, where at least one of them has been identified as a muon by the muon system. Requiring muon identification for both tracks significantly reduces the background, at the expense of losing approximately half the signal (see fig. 5.5). This is due to the fact that of all the signal events which contained at least one muon candidate, only half contained two or more (see fig. 5.6). The invariant mass of the $J / \psi$ has a resolution of 0.047 $\mathrm{GeV}$.

Fig. 5.7 shows the $p_{t}$ spectrum of the simulated $J / \psi$ and the $p_{t}$ spectrum of all $J / \psi$ candidates (with a one muon requirement) which could be matched to an $\mathrm{MC} J / \psi$. The $J / \psi$ momentum resolution is shown in fig. 5.8.

A common vertex for the two tracks is found using a Kalman fitter [68]. The $\chi^{2}$ distribution of the $J / \psi$ candidates is shown in fig. 5.9. The goal of the measurement is to measure the $B$-meson lifetime which requires the reconstruction of the $B$ decay vertex. To reduce combinatorics $J / \psi$ candidates with a $\chi^{2}>10$ are discarded even if they pass the $J / \psi$ mass cut of $m_{J / \psi}^{\mathrm{PDG}} \pm 0.09 \mathrm{GeV}(\approx 2 \sigma)$.

It is possible to enhance the $J / \psi$ signal by using the $J / \psi \rightarrow e^{+} e^{-}$channel. While a $J / \psi \rightarrow e^{+} e^{-}$signal has been established in real data [69], there is no trigger for this channel available and while studies are underway it is unlikely that it will contribute to physics measurements in the near future. 


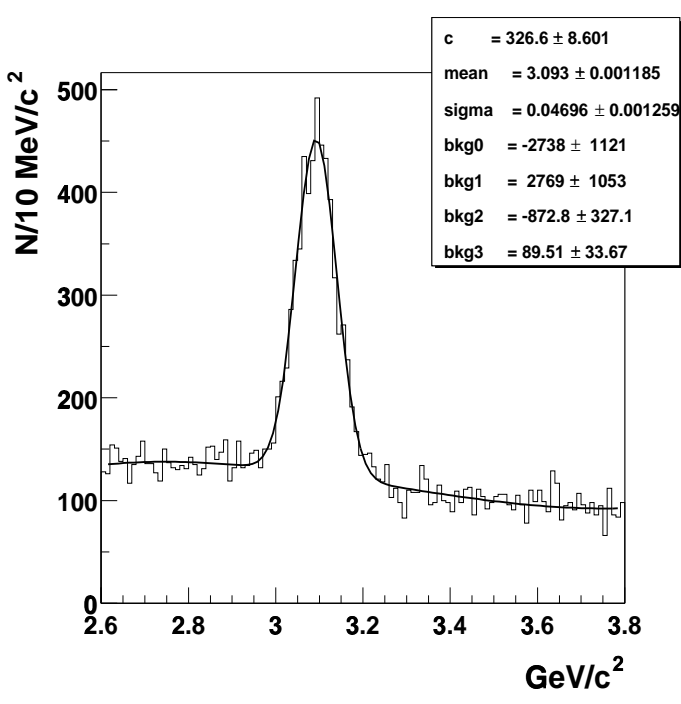

(a) one identified $\mu$ required

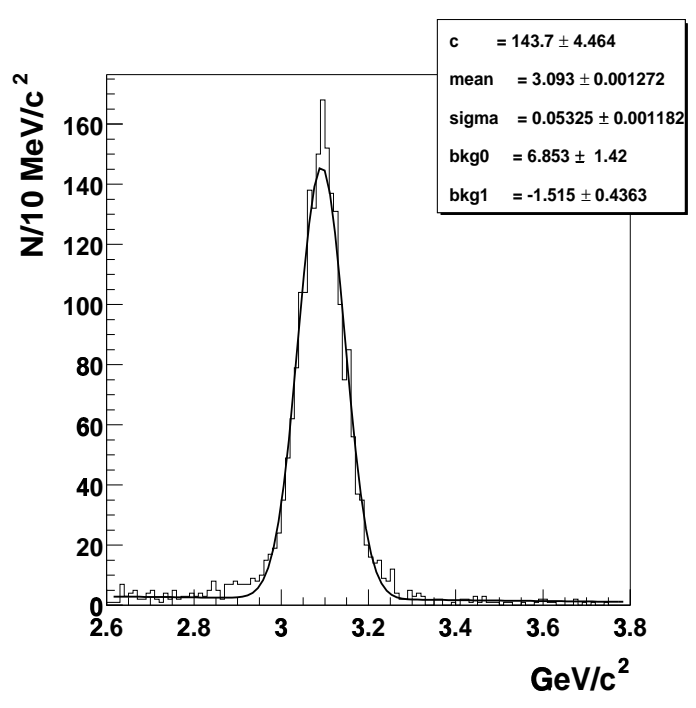

(b) two identified $\mu$ required

Figure 5.5: Invariant mass distribution for all $J / \psi$ candidates in the signal sample. The fitted function in (a) is a Gaussian and a third order polynomial, the function in (b) a Gaussian and a straight line. The peak in (a) contains approximately twice as many $J / \psi$ candidates as the peak in (b).

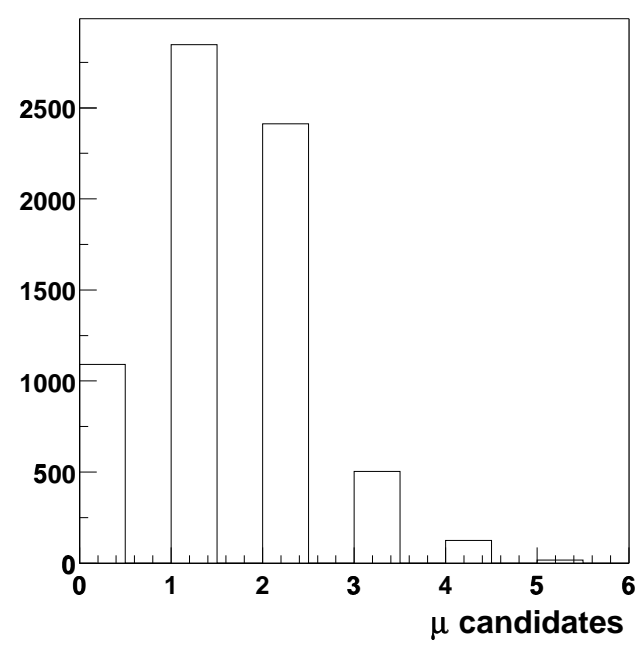

Figure 5.6: Number of muon candidates per event. Of the 7000 signal events 1091 did not contain any muon candidates, 2847 contained one candidate and 3062 contained two or more muon candidates. 


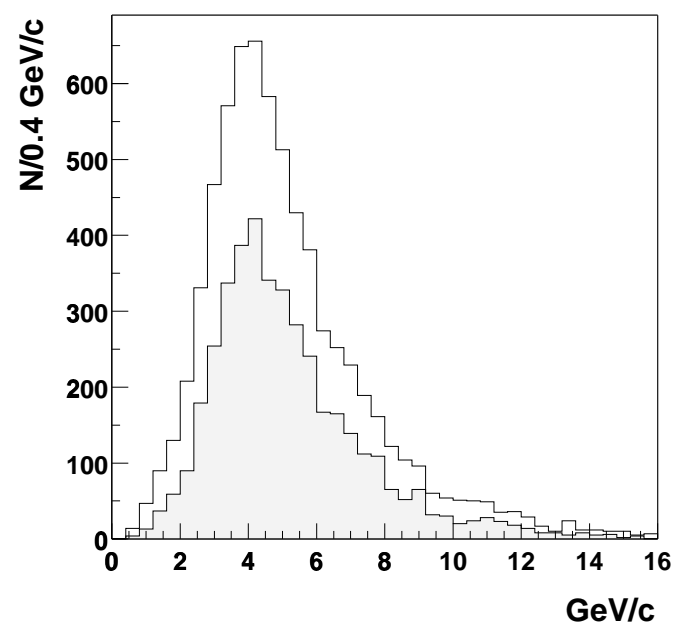

Figure 5.7: Transverse momentum for simulated (open histogram) and matched (shaded histogram) $J / \psi$. The $J / \psi$ candidates were required to have at least one identified muon.

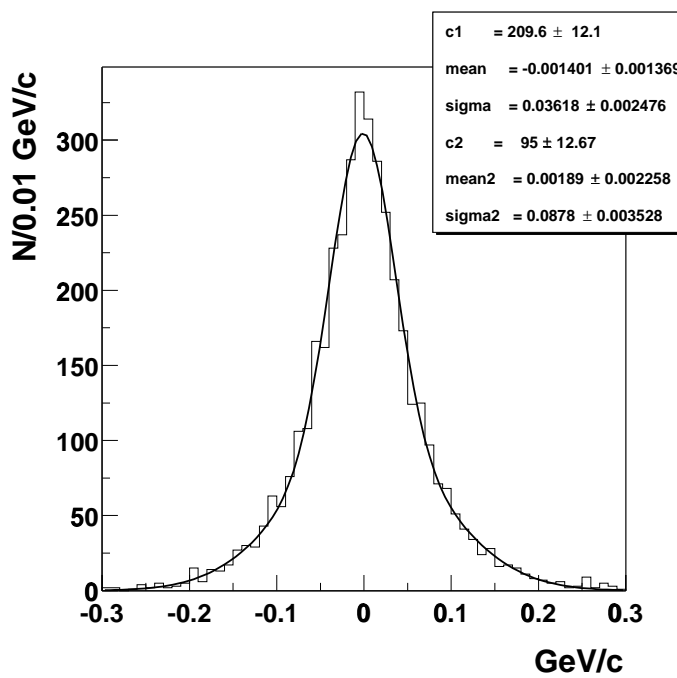

(a) $p_{x}$

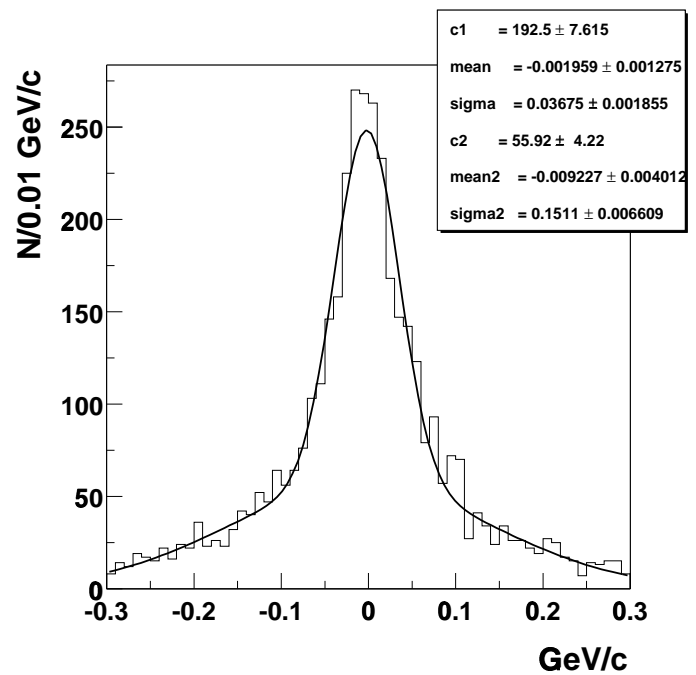

(b) $p_{z}$

Figure 5.8: $J / \psi$ momentum resolution in $p_{x}$ and $p_{z}$. The fitted function is a double Gaussian. 


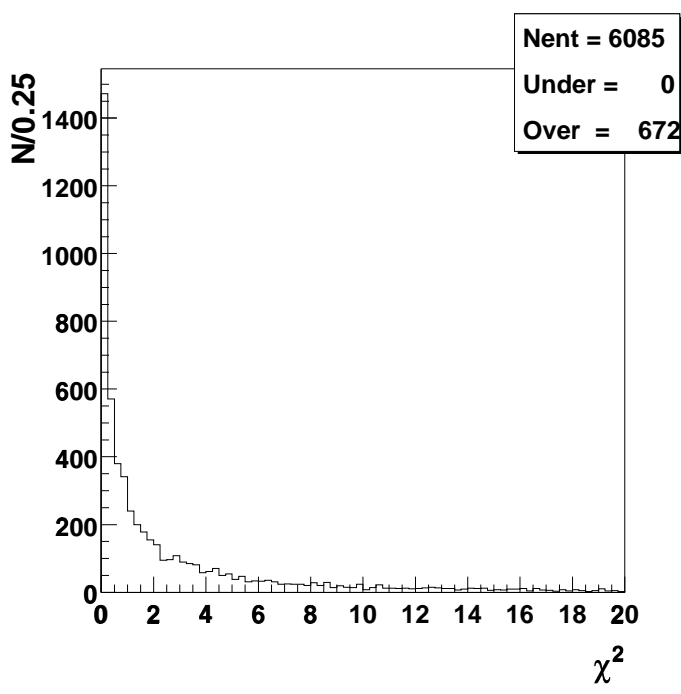

(a) one identified $\mu$

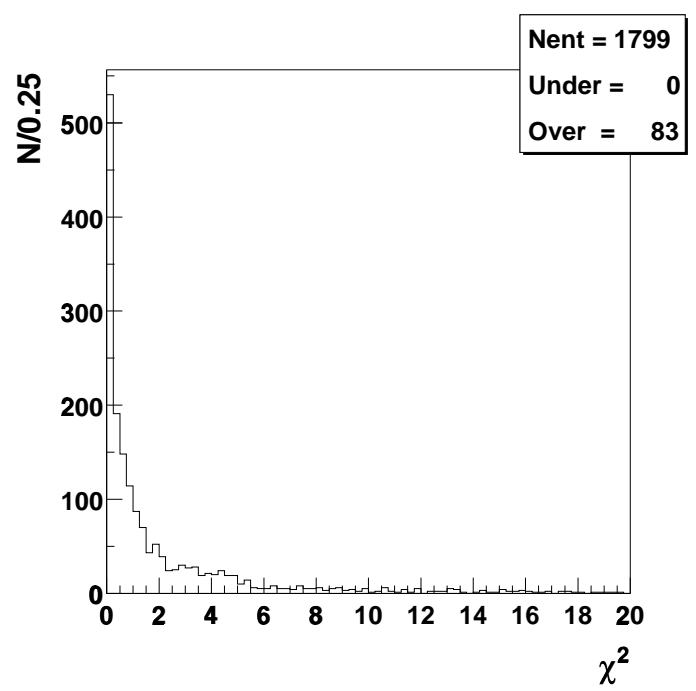

(b) two identified $\mu$

Figure 5.9: $J / \psi \quad \chi^{2}$ distribution for candidates with an invariant mass of $m^{\text {reco }}=m_{J / \psi}^{\mathrm{PDG}} \pm 0.09 \mathrm{GeV}$. The two track fit has one degree of freedom.

\subsection{3 $\phi$ reconstruction}

As $D \varnothing$ has no particle identification apart from its muon chambers, $\phi$ candidates are formed by combining opposite charged tracks which are not identified as muons. The fact that the $\phi$ has a very small natural width $(\Gamma=4.458 \pm 0.032 \mathrm{MeV})$ allows for a significant background reduction when combining a $\phi$ candidate with $J / \psi$ candidate to form a $B_{s}^{0}$. Fig. 5.10 shows the reconstructed $\phi$ mass in the signal sample. The signal resolution is dominated by the natural width of the $\phi$. The $\phi$ mass resolution itself is approximately $7 \mathrm{MeV}$.

Most reconstructed $\phi$ candidates have a $p_{t}>1.5 \mathrm{GeV}$, while the $p_{t}$ of the background in the $\phi$ mass range peaks around $1 \mathrm{GeV}$ (see fig. 5.11). In order to reduce background therefore a minimum $p_{t}$ of $1.5 \mathrm{GeV}$ is required for all $\phi$ candidates. 


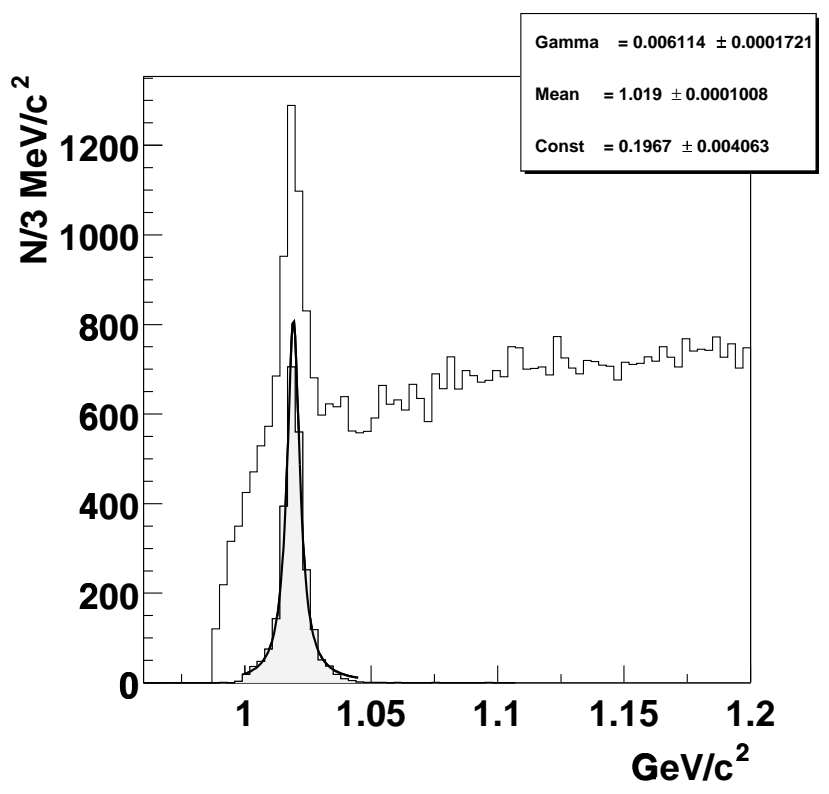

Figure 5.10: $\phi$ mass from opposite charged tracks (signal sample only). The shaded histogram is the invariant mass of all $\phi$ candidates which could be matched to an MC $\phi$. The fitted function is a Breit-Wigner distribution.

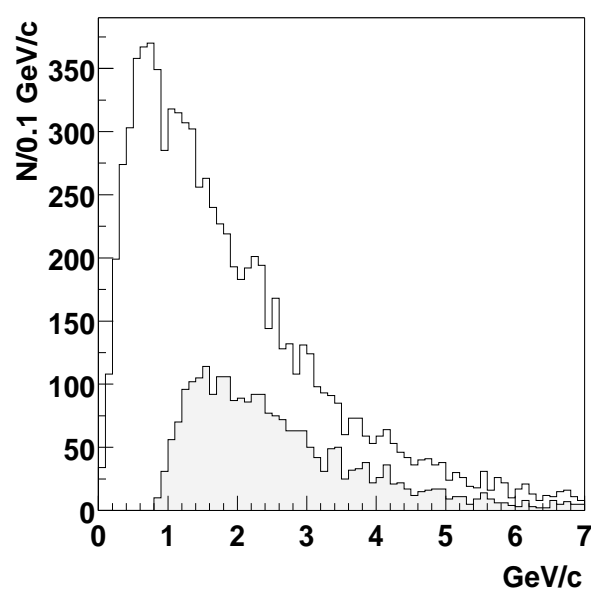

(a) signal

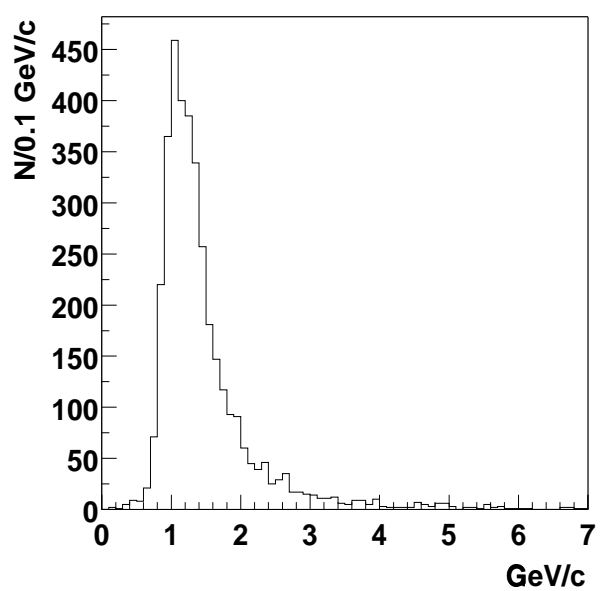

(b) background

Figure 5.11: (a) MC transverse momentum for all simulated $\phi \rightarrow K^{+} K^{-}$. The shaded histogram is the MC momentum for all matched $\phi$ candidates. (b) $p_{t}$ distribution for unmatched $\phi$ candidates passing the $\phi$ mass cut $\left(\left|m_{\phi}^{\mathrm{PDG}}-m_{\phi}^{\mathrm{reco}}\right|<0.01 \mathrm{GeV}\right)$. 


\subsection{4 $\mathrm{B}_{\mathrm{s}}^{0}$ reconstruction}

A $B_{s}^{0}$ candidate is formed by combining a $J / \psi$ and a $\phi$ candidate that passed their respective cuts. The invariant mass is calculated and the four tracks are fitted to a common vertex using a Kalman fitter. A loose $\chi^{2}$ cut of 50 is applied to remove track combinations where the tracks obviously originate at different points in the detector. An overview of all cuts is given in table 5.2.

The $B_{s}^{0}$ mass resolution is $0.028 \mathrm{GeV}$. Figs. 5.12 and 5.13 show the invariant mass of the $B^{0}$ candidates for two different sets of cuts. In fig. 5.12 only a minimum number of cuts is applied ( $J / \psi$ and $\phi$ mass cuts and $J / \psi$ vertex cut), while fig. 5.13 shows the result after applying all cuts.

The $B_{s}^{0}$ mesons were produced with a minimum $p_{t}$ cut at generator level of $3.0 \mathrm{GeV}$. The same cut is applied in the reconstruction. As fig. 5.14 shows the $p_{t}$ of the reconstructed $B_{s}^{0}$ candidates peaks around 5-6 GeV and falls off towards lower $p_{t}$. If there is more than one $B_{s}^{0}$ candidate in the event ${ }^{7}$, candidates where the $J / \psi$ is made of two identified muons are given preference. If there is no such candidate or if there is more more than one, then the candidate with the lowest $\chi^{2}$ is kept. The $\chi^{2}$ distribution of the remaining candidates is shown in fig. 5.15.

\footnotetext{
${ }^{7}$ The chance that an event contains a $B_{s}^{0}$ and a $\bar{B}_{s}^{0}$ which both decay to $J / \psi \phi$ is tiny $\left(\sim 10^{-10}\right)$.
} 


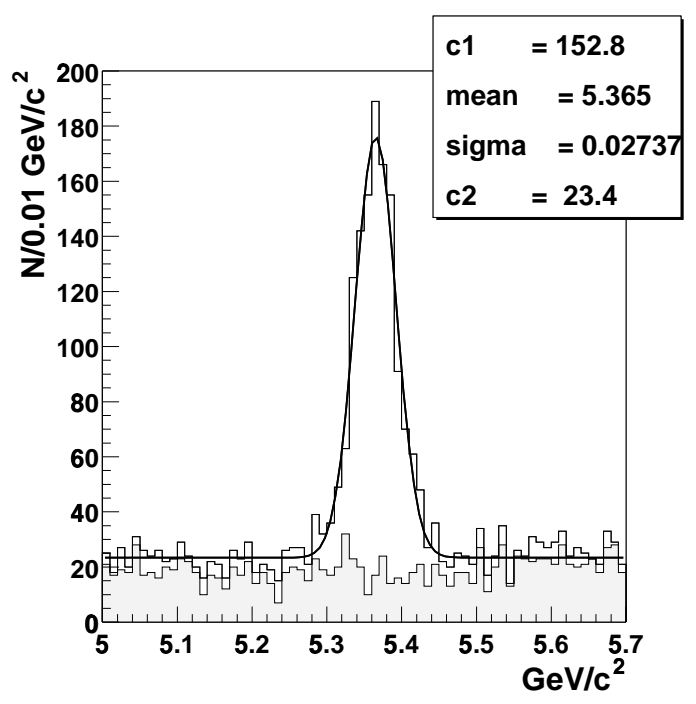

Figure 5.12: Invariant mass of all $B_{s}^{0}$ candidates after applying the $J / \psi$ and $\phi$ mass and the $J / \psi$ $\chi^{2}$ cuts. The open histogram shows all candidates found in the complete sample (63500 events). The shaded histogram contains all candidates found in the background sample. A Gaussian and a constant were fitted. The peak contains approximately $1000 B_{s}^{0}$ candidates.

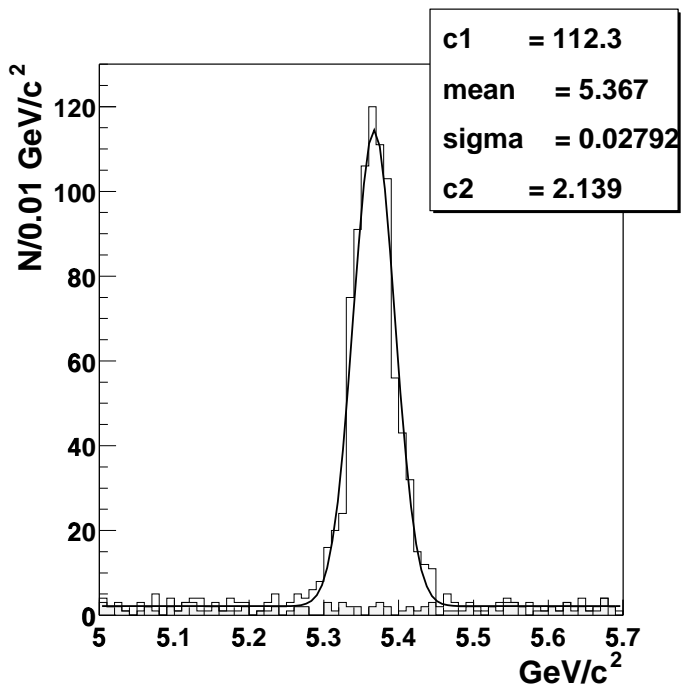

Figure 5.13: Invariant mass of all $B_{s}^{0}$ candidates passing all cuts (cf. table 5.2). The open histogram shows all candidates found in the complete sample. The shaded histogram are all candidates found in the background sample. A Gaussian and a constant were fitted. The peak contains approximately $780 B_{s}^{0}$ candidates. 

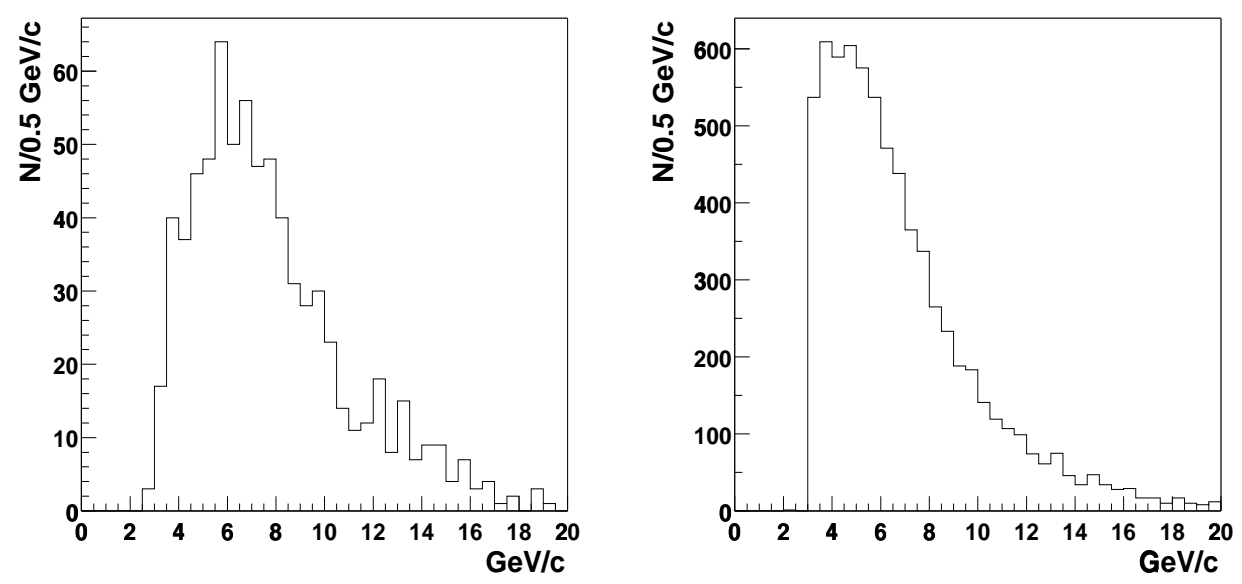

(a) reconstructed

(b) generated

Figure 5.14: Transverse momentum for generated and reconstructed $B_{s}^{0}$.

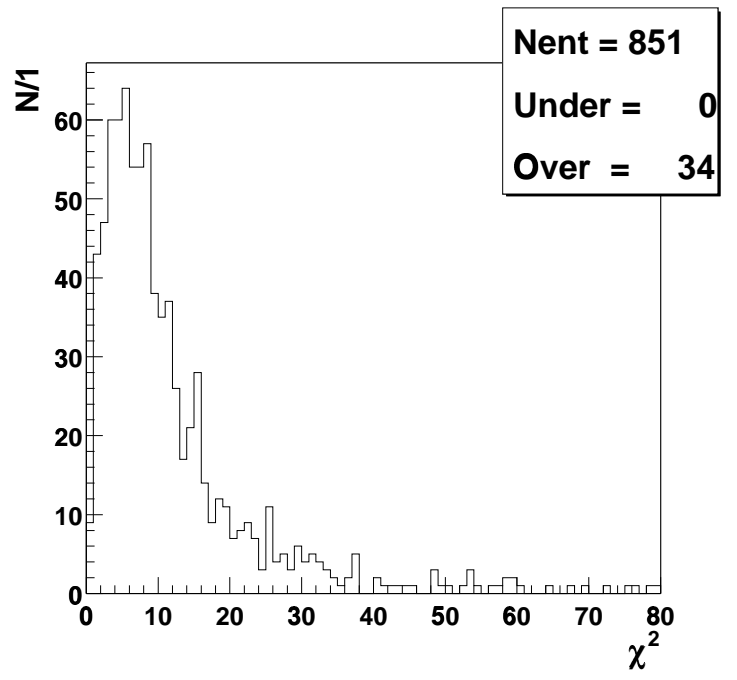

Figure 5.15: $\chi^{2}$ distribution for selected $B_{s}^{0}$ candidates within $0.06 \mathrm{GeV}(\approx 2 \sigma)$ of the nominal $B_{s}^{0}$ mass. The four track fit has five degrees of freedom. Candidates had to pass all cuts listed in table 5.2, except for the $B_{s}^{0} \chi^{2}$ cut. If more than one candidate per event passed all cuts, only the candidate with the smallest $\chi^{2}$ was kept. 


\begin{tabular}{|l|l|}
\hline cut & reference \\
\hline \hline$\left|m_{J / \psi}-m_{J / \psi}^{\mathrm{PDG}}\right|<0.09 \mathrm{GeV}$ & $2 \sigma$ of $J / \psi$ mass resolution (fig. 5.5$)$ \\
\hline$J / \psi$ fit $\chi^{2}<10.0$ & see fig. 5.9 \\
\hline$\left|m_{\phi}-m_{\phi}^{\mathrm{PDG}}\right|<0.01 \mathrm{GeV}$ & see fig. 5.10 \\
\hline$p_{t}$ of $\phi$ candidate $>1.5 \mathrm{GeV}$ & see fig. 5.11 \\
\hline $\begin{array}{l}\text { prefer } B_{s}^{0} \quad \text { candidates with two } \\
\text { identified muons }\end{array}$ & less fake $J / \psi$ (fig. 5.5) \\
\hline$p_{t}$ of $B_{s}^{0}$ candidate $>3.0 \mathrm{GeV}$ & see fig. 5.14 \\
\hline$\chi^{2}$ four track fit $<50.0$ & see fig. 5.15 \\
\hline $\begin{array}{l}\text { choose candidate with smallest } \chi^{2} \text { in } \\
\text { four track fit }\end{array}$ & $\begin{array}{l}\text { all tracks should be originating at the } \\
\text { same point (cf. section 2.7) }\end{array}$ \\
\hline \hline $\begin{array}{l}\text { distance between primary vertex and } \\
\text { secondary vertex }<2 \mathrm{~cm}\end{array}$ & unphysical \\
\hline$\left|m_{B_{s}^{0}}-m_{B_{s}^{0}}^{\text {PDG }}\right|<0.06 \mathrm{GeV}$ & $2 \sigma$ of $B_{s}^{0}$ mass resolution (fig. 5.13) \\
\hline
\end{tabular}

Table 5.2: Summary of cuts used for $B_{s}^{0}$ reconstruction. The last two cuts are only used in the lifetime fit.

\subsubsection{Vertex reconstruction}

Apart from the main interaction, each bunch crossing in the detector can produce a number of minimum bias events which are recorded together with the main event. The number of minimum bias events depends on the luminosity (fig. 5.16). In the $\mathrm{MC}$ sample the average number of minimum bias events was set to 0.5 .

Primary vertices are initially found in a tear down approach [70] using all tracks with a transverse impact parameter significance ${ }^{8}$ smaller than 3 to exclude tracks from secondary vertices. All tracks that pass the cut are fitted together and the $\chi^{2}$ contribution of each track is calculated. The track with the highest $\chi^{2}$ is excluded and the fit repeated until all the remaining tracks have a $\chi^{2}$ contribution of less than 10. This procedure is then repeated with the unused tracks to find further primary

\footnotetext{
${ }^{8}$ The transverse impact parameter significance is defined as the distance of closest approach of a track to the origin $(0,0)$ in the $x-y$ plane (nominal beam position) divided by its resolution. Large impact parameters are associated with secondary vertices.
} 


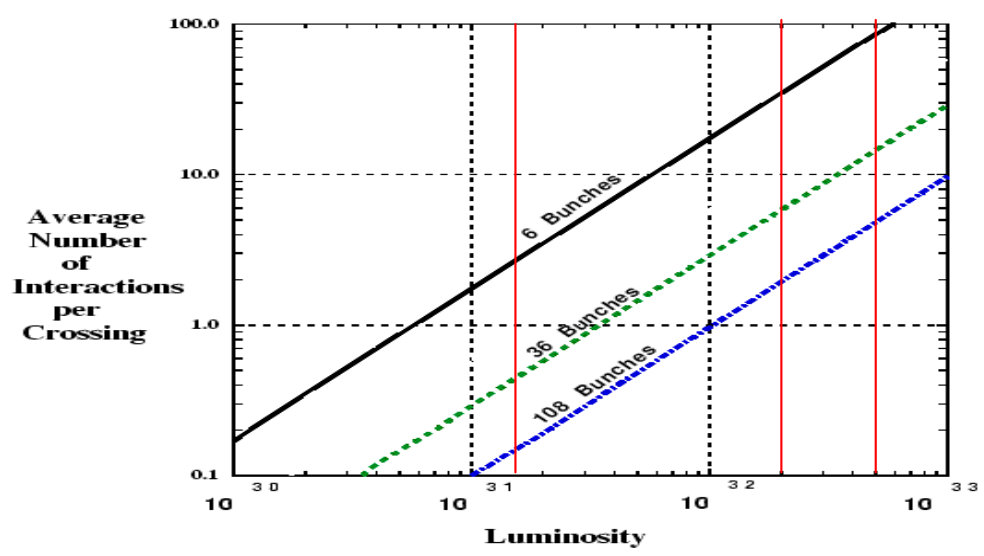

Figure 5.16: Number of minimum bias events as a function of the luminosity and the number of bunches.

vertices. If more than one primary vertex is found all the vertices are kept, but the vertex with highest $\sum \log p_{t}$ is selected as the primary vertex corresponding to the hard-scatter rather than the minimum bias event.

As the main interaction and the minimum bias vertices are usually several centimeters apart, multiple primary vertices do not pose a problem for the lifetime measurement. If the vertex reconstruction finds several primary vertices, the one closest to the reconstructed $B$-meson decay vertex (secondary vertex) is chosen. Events where there is no primary vertex within $2 \mathrm{~cm}$ of the secondary vertex are discarded.

Another, albeit small, problem results from the fact that the impact parameter cut is often not sufficient to exclude tracks originating from $B$-meson decays. This introduces a slight bias towards the secondary vertex. This bias can be shown by calculating the projection of the vertex residual onto the Monte Carlo $B$-momentum (see fig. 5.17).

Fig. 5.18 shows the projected residual $\Delta \vec{r} \cdot \vec{p}_{B}$ for the original primary vertex and for a refitted vertex after removing all tracks used to form the secondary vertex. The original primary vertex shows a (positive) bias towards the secondary vertex. Refitting reduces this bias from about $14 \mu \mathrm{m}$ to about $2 \mu \mathrm{m}$. For all lifetime mea- 


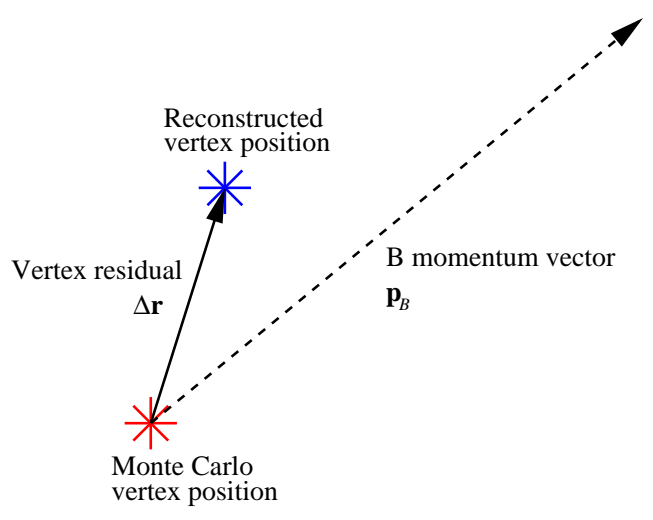

Figure 5.17: The reconstructed and MC primary vertex and the MC $B$-momentum.

surements the refitted vertex is used.

Figs. 5.19 and 5.20 show the resolutions in $x$ and $z$ for all the primary vertices in the signal sample and fig. 5.21 for the secondary vertices. In the case of the primary vertex two Gaussians were fitted to the distribution. The $\sigma$ of the inner Gaussians are $32 \mu \mathrm{m}$ in $x$ and $38 \mu \mathrm{m}$ in $z$. The resolutions for the secondary vertices, which were fitted with a single Gaussian, are $40 \mu \mathrm{m}$ in $\mathrm{x}$ and $50 \mu \mathrm{m}$ in $z$.

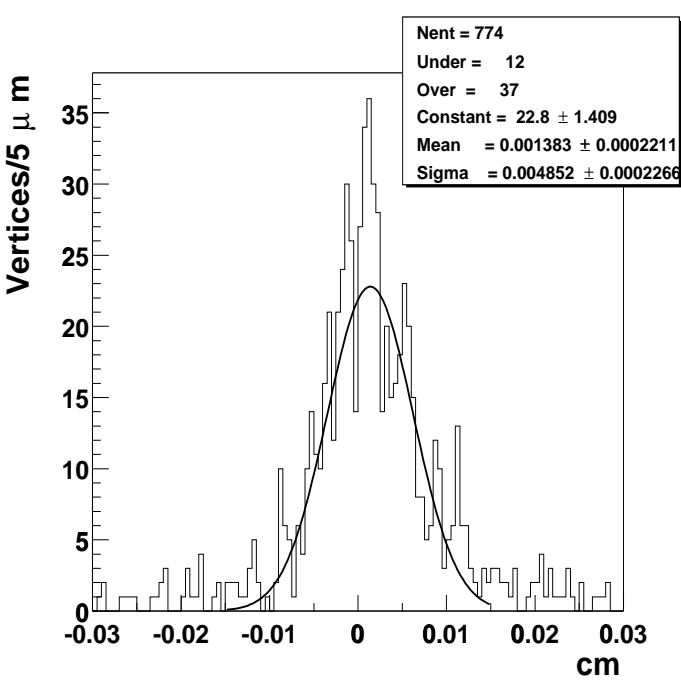

(a) initial vertex

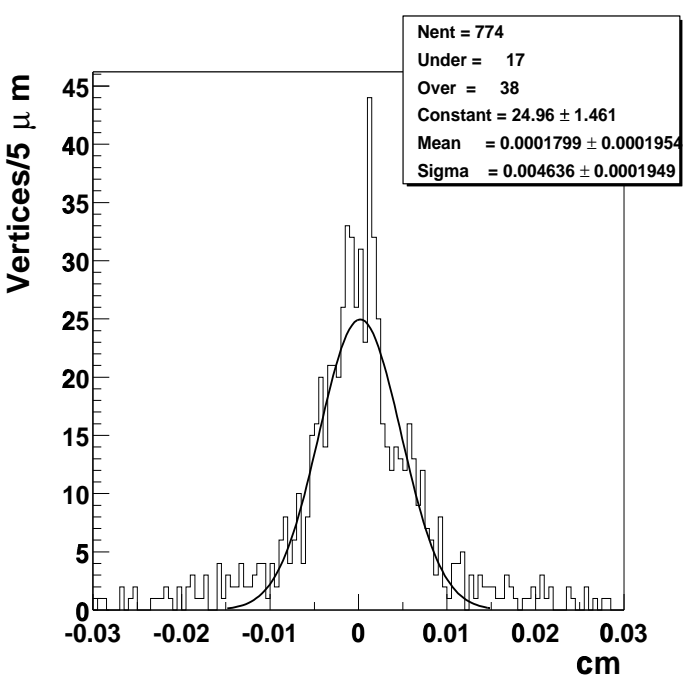

(b) refitted vertex

Figure 5.18: Projected residual for the primary vertex. 


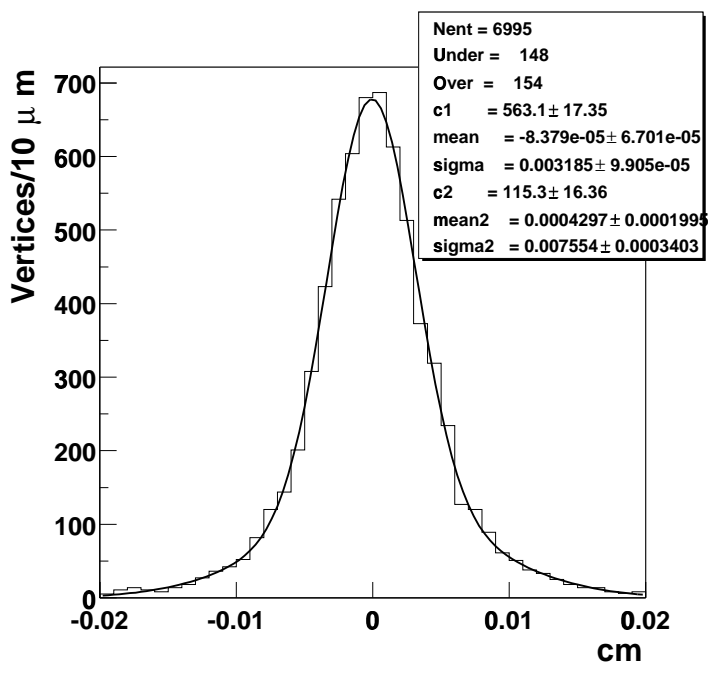

Figure 5.19: Primary vertex resolution (MC-vertex - reconstructed vertex) in $x$. The sample contained 7000 events. In five events no primary vertex was found. Two Gaussians were fitted to the distribution.

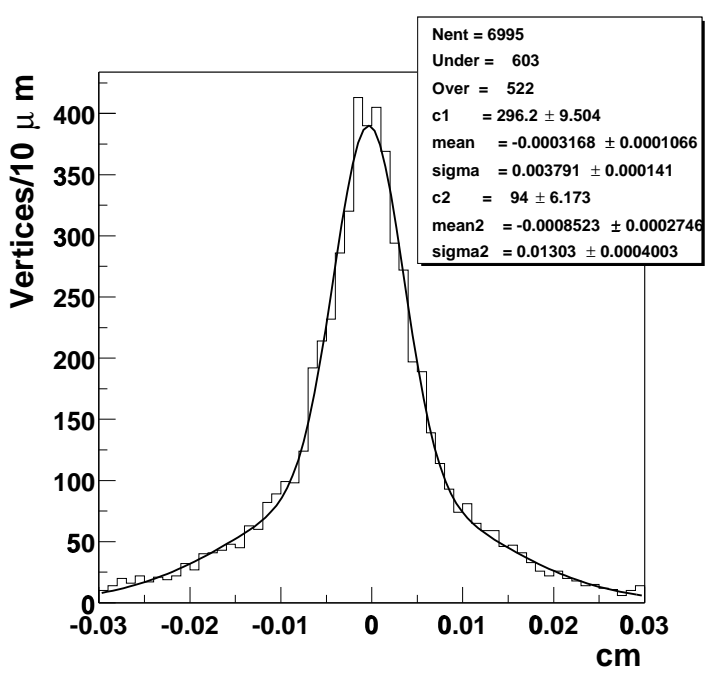

Figure 5.20: Primary vertex resolution (MC-vertex - reconstructed vertex) in $z$. Two Gaussians were fitted to the distribution. 


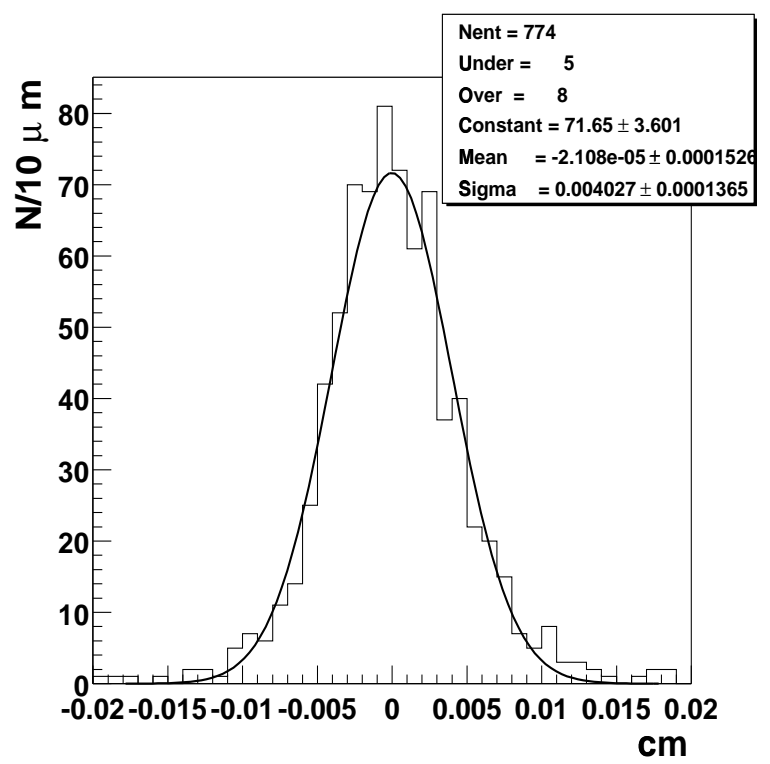

(a) resolution in $x$

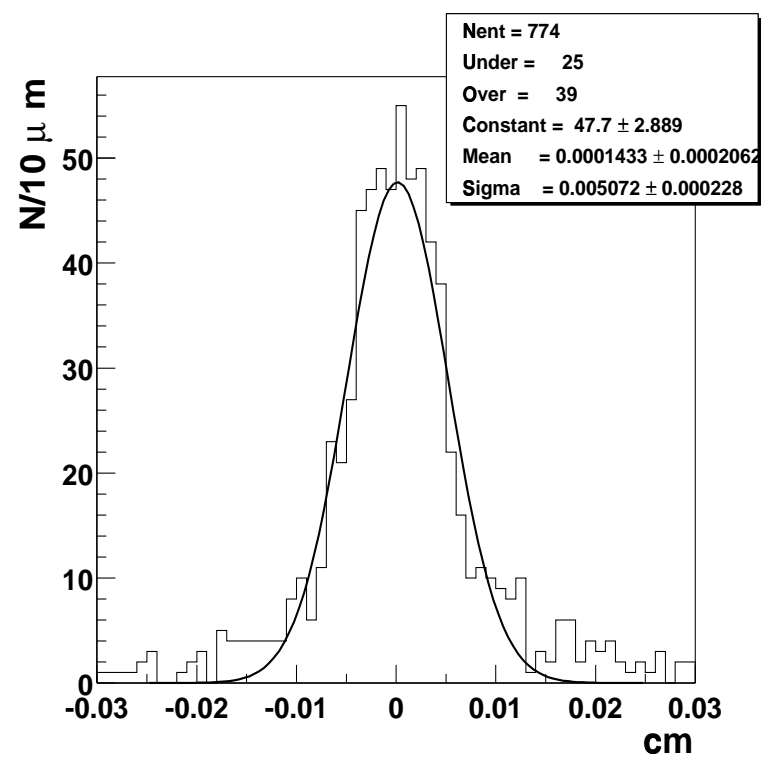

(b) resolution in $z$

Figure 5.21: $B_{s}^{0}$ secondary vertex resolution with a fitted Gaussian. 


\subsubsection{Lifetime}

The decay length $d$ in the laboratory frame is the distance between the secondary and primary vertex position:

$$
\vec{d}=\vec{x}_{\text {primary }}-\vec{x}_{\text {secondary }}
$$

It is related to the proper decay length $L$ by

$$
\vec{d}=\vec{\beta} \gamma L
$$

where $\vec{\beta}$ is the velocity of the $B$-meson and $\gamma=1 / \sqrt{1-\beta^{2}}$. Using $\vec{p}=\vec{\beta} \gamma m_{B}$ gives

$$
\vec{d}=\frac{\vec{p}}{m_{B}} L
$$

The proper lifetime and proper decay length only differ by a factor of $c$ and are used interchangeably.

Equation 5.3 does not take into account that the reconstructed decay length $\vec{d}$ and the $B$-momentum $\vec{p}$ will not always be proportional (or parallel) to each other. When measuring a lifetime, therefore the projection of the decay length vector $\vec{d}$ onto the $B$-momentum is used:

$$
L=\frac{\vec{d} \cdot \vec{p}}{p^{2}} \cdot m_{B}
$$

$L$ is a signed variable. A negative value indicates that $\vec{p}$ and $\vec{d}$ point in 'opposite' directions and the $B$-meson seems to decay before it was produced. This is especially common for short decay times: For a particle with zero lifetime, $L$ should result in a Gaussian distribution peaked at zero. 
The error on the lifetime is

$$
\sigma^{2}(L)=\frac{m_{B}}{p^{4}}\left[\vec{p}^{T} V_{d} \vec{p}+\left(\vec{d}-\frac{2(\vec{d} \cdot \vec{p})}{p^{2}} \vec{p}\right)^{T} V_{p}\left(\vec{d}-\frac{2(\vec{d} \cdot \vec{p})}{p^{2}} \vec{p}\right)\right]+(\vec{d} \cdot \vec{p})^{2} \sigma^{2}\left(m_{B}\right)
$$

where $V_{d}$ and $V_{p}$ are the covariance matrices of $\vec{d}$ and $\vec{p}$ and $\sigma\left(m_{B}\right)$ is the error on the $B$-meson mass. A derivation of eq. 5.5 can be found in Appendix A.3. Both the $B_{s}^{0}$ and the $B_{d}^{0}$ masses are known to an accuracy of $0.05 \%$ (see table 5.1 ), and therefore their contribution is negligible. The main contribution to the error in the lifetime measurement is the error in the vertex measurement.

As well as the full three-dimensional decay length the so-called transverse decay length $L_{x y}$ has been used in lifetime measurements [71]. Here, instead of using the full decay length and $B$ momentum, only the transverse components are used, which leads to

$$
L_{x y}=\frac{\overrightarrow{d_{t}} \cdot \vec{p}_{t}}{p_{t}^{2}} m_{B}
$$

Using $L_{x y}$ instead of $L$ can be an advantage if the vertex resolution in $x$ and $y$ is considerably better than in $z$. According to figs. 5.19, 5.20, and 5.21 this is not the case here and, as figs. 5.22(a) and 5.23(a) show, the lifetime resolution for the two and three-dimensional case are comparable. Figs. 5.22(b) and 5.22 (b) show the difference between the $\mathrm{MC}$ and the reconstructed proper decay length divided by its error. $\mathrm{A} \sigma$ of $\approx 1.4$ indicates that the calculated error values are too small, but not completely unreasonable. When performing a fit on the data this will be taken into account by allowing a scale factor for the error to float with the fit. 


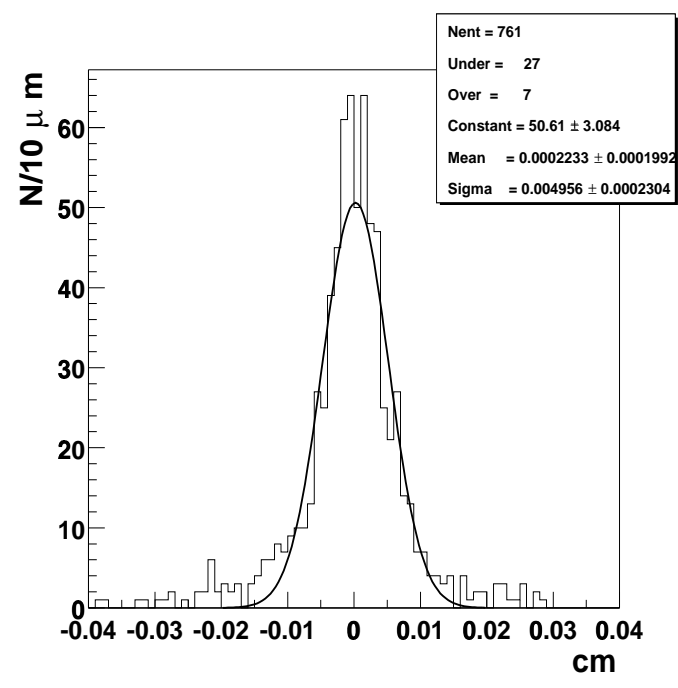

(a)

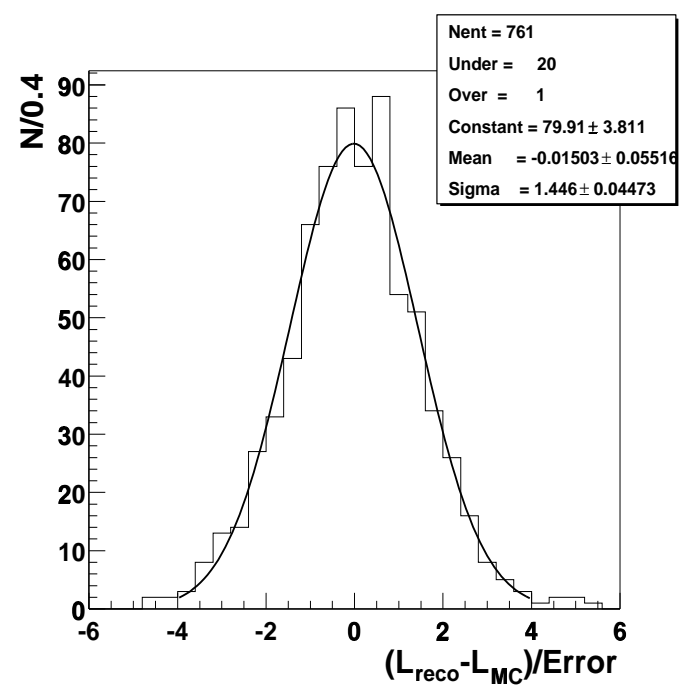

(b)

Figure 5.22: (a) Proper decay length resolution (reconstructed proper decay length-MC proper decay length) for $B_{s}^{0} \rightarrow J / \psi \phi$. If the event was a background event the MC proper decay length was set to 1000.0 which causes the excess of events in the underflow bin. (b) Difference between the MC proper decay length and the reconstructed proper decay length divided by the error on

the proper decay length according to eq. 5.5. In both histograms a Gaussian was fitted.

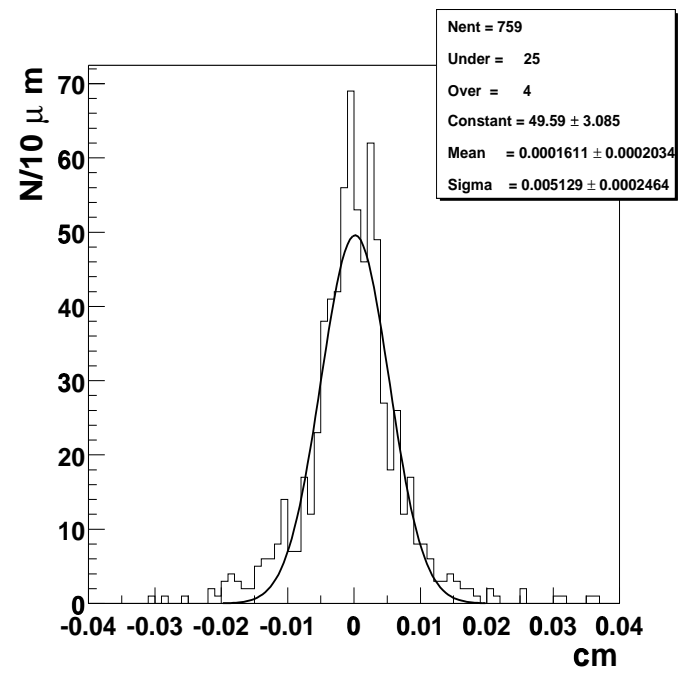

(a)

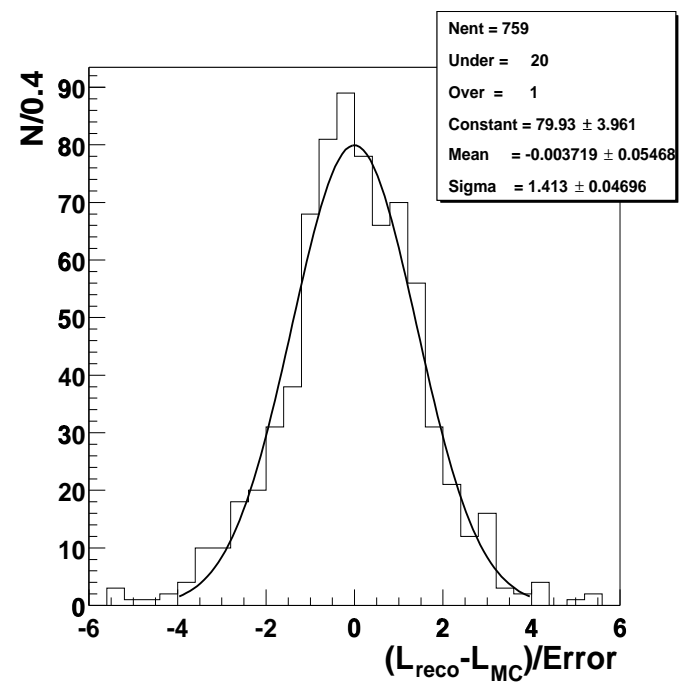

(b)

Figure 5.23: (a) Transverse decay length resolution. (b) Difference between the MC transverse proper decay length and the reconstructed transverse proper decay length divided by its error. 


\subsubsection{Fitting}

In order to extract the $B$-meson lifetime, an unbinned maximum likelihood fit is performed on the data. A brief description of this technique is given in Appendix A.5. It also lists all probability density functions used in full.

The probability density function for the lifetime of a particle is an exponential decay. The error on each measurement is taken into account by folding this exponential with a Gaussian. The resulting function $\mathcal{F}$ is given in eq. A.30.

The background is assumed to be a Gaussian peak $\mathcal{G}$ around zero with a nonGaussian tail on the positive side. This tail is mainly caused by other long-lived particles $\left(D, \Lambda_{b}\right)$ that are mis-reconstructed as $B$-mesons. The fractions of background events in the tail and the peak are denoted by $f_{+}$and $\left(1-f_{+}\right)$.

The likelihood function $\mathcal{L}$ for each event is then

$$
\mathcal{L}_{i}=N_{\text {sig }} \mathcal{F}\left(L_{i}, \sigma_{i} \mid \lambda\right)+N_{\text {bg }} \mathcal{L}_{\text {bg }}
$$

where $N_{\text {sig }}$ is number of signal and $N_{\text {bg }}$ the number of background events. The $L_{i}$ are the reconstructed lifetimes with their errors $\sigma_{i}$ and $\lambda$ is the $B$-meson lifetime. The likelihood function $\mathcal{L}_{\text {bg }}$ for the background is given by

$$
\mathcal{L}_{\mathrm{bg}}=\left(1-f_{+}\right) \mathcal{G}\left(L_{i}, \sigma_{i}\right)+f_{+} \frac{e^{-L_{i} / \lambda_{+}}}{\lambda_{+}}
$$

The extended likelihood fit then maximises

$$
\sum_{i} \log \mathcal{L}_{i}-N_{\mathrm{sig}}-N_{\mathrm{bg}}
$$

The sample itself is divided in a background ('sidebands') and signal part. Any $B_{s}^{0}$-candidate with $\left|m_{B_{s}^{0}}^{\mathrm{reco}}-m_{B_{s}^{0}}^{\mathrm{PDG}}\right|<0.06 \mathrm{GeV}$ is defined to be in the signal region. The sidebands comprise masses between $\left|m_{B_{s}^{0}}^{\text {reco }}-m_{B_{s}^{0}}^{\mathrm{PDG}}\right|>0.06 \mathrm{GeV}$ and $\left|m_{B_{s}^{0}}^{\mathrm{reco}}-m_{B_{s}^{0}}^{\mathrm{PDG}}\right|<0.4 \mathrm{GeV}$. The signal region still contains background events, while the sidebands are supposed to be background only. The shape of the background distribution can be found by fitting the background part $\mathcal{L}_{\mathrm{bg}}$ of the likelihood func- 
tion to the sidebands. It is assumed that it will have the same shape in the signal region. For practical purposes this fit was implemented as an extended likelihood fit as well, even though it only contains one component. The fitting routine then simultaneously fits the background likelihood function to the sidebands and the full likelihood function to the signal region. The proper decay length distributions for the signal region and the sidebands are shown in figs. 5.24 and 5.25.

The results from the full fit are given in table 5.3. The 7000 signal events correspond to $\sim 0.12 \mathrm{fb}^{-1}$ of data. The fit finds a $B_{s}^{0}$ lifetime of $1.488 \mathrm{ps}(\mathrm{c} \tau=446 \mu \mathrm{m})$ with an error of 0.063 ps. The simulated lifetime was 1.464 ps $(439 \mu \mathrm{m})$. The current error [3] on the $B_{s}^{0}$ lifetime is $0.062 \mathrm{ps}$. The real measurement on $2 \mathrm{fb}^{-1}$ will suffer from more background events, but will also have higher statistics. As long as it it possible to model the lifetime distribution of the background events correctly $D \varnothing$ should be able to improve the current measurement in this channel.

\begin{tabular}{|l|c|}
\hline$\lambda_{B_{s}^{0}}$ Lifetime of $B_{S}^{0}$ & $1.488 \pm 0.0063 \mathrm{ps}$ \\
Error scale factor & $1.78 \pm 0.11$ \\
$\lambda_{+}$Lifetime of positive tail & $1.187 \pm 0.127 \mathrm{ps}$ \\
$f_{+}$Fraction of background events in positive tail & $0.69 \pm 0.05$ \\
Number of signal events & $701 \pm 39$ \\
Number of background events in signal region & $59 \pm 30$ \\
Number of events in sideband & $191 \pm 14$ \\
\hline
\end{tabular}

Table 5.3: Results from the unbinned maximum likelihood fit for the $B_{s}^{0}$ lifetime. The $B_{s}^{0}$ was simulated with a lifetime of 1.464 ps. 


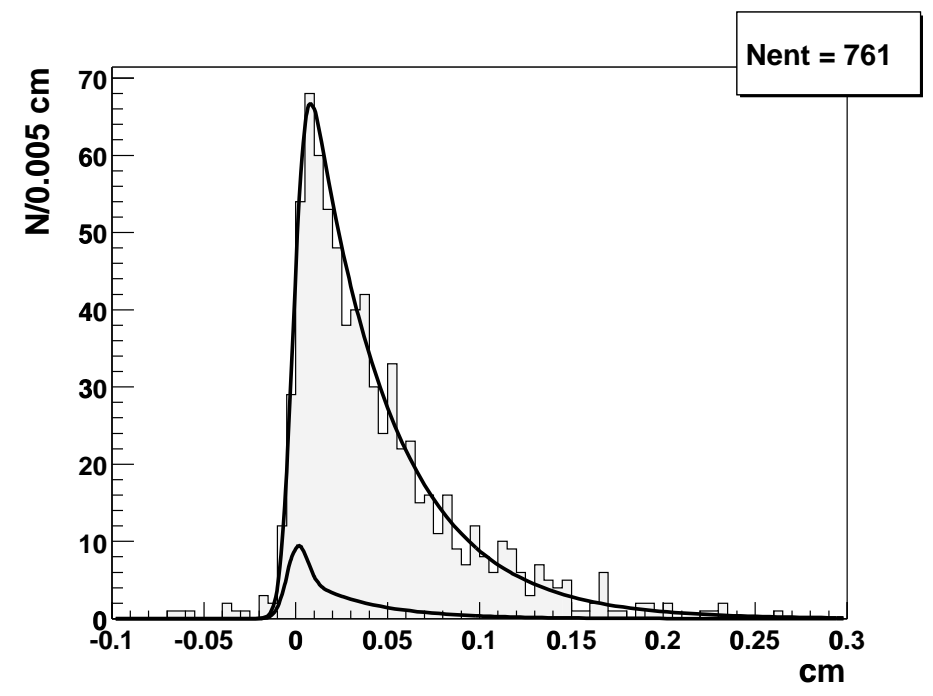

Figure 5.24: Proper decay length distribution for events in the signal region. Note that the fit was an unbinned likelihood fit, so the fit function is plotted with a fixed value for the resolution, while during the fit the individual error for each entry is used. The upper curve is the full likelihood function (signal and background), the lower curve is the background contribution in the signal region.

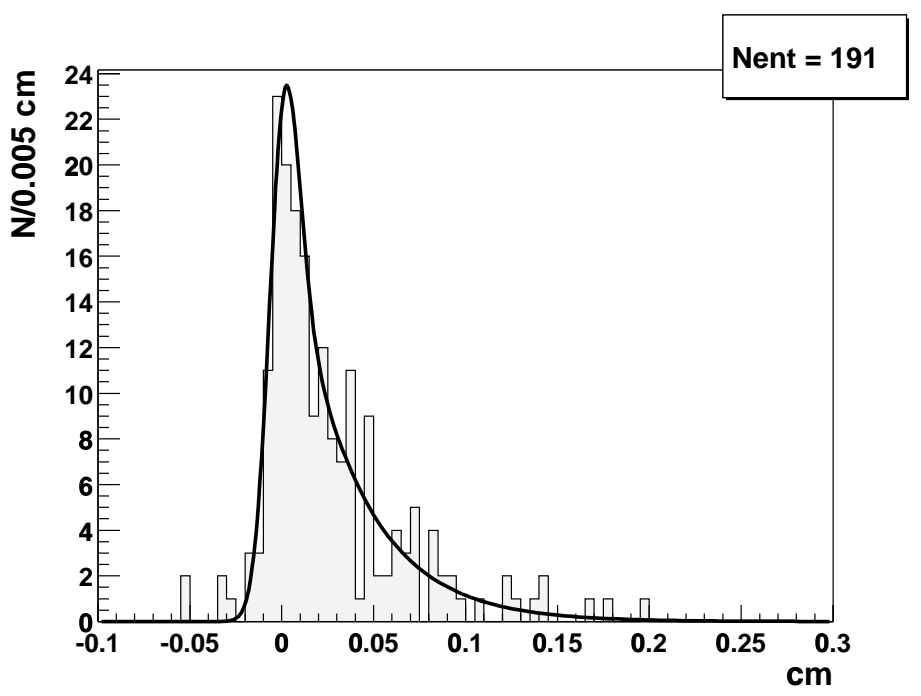

Figure 5.25: Proper decay length distribution and fitted function in the sidebands. 


\section{$5.4 \quad B_{d}^{0} \rightarrow J / \psi\left(\rightarrow \mu^{+} \mu^{-}\right) K^{* 0}\left(\rightarrow K^{+} \pi^{-}\right)$}

The reconstruction of $B_{d}^{0} \rightarrow J / \psi K^{* 0}$ is very similar to $B_{s}^{0} \rightarrow J / \psi \phi$ with the $K^{* 0}$ taking the place of the $\phi$. In fact both channels will always contain a small contribution of each other as background (section 5.5).

The lack of particle identification adds a complication to the reconstruction of the $K^{* 0}\left(\rightarrow K^{+} \pi^{-}\right)$as there are now two possibilities to assign the $K$ and the $\pi$ to each combination of opposite charged tracks. As fig. 5.26 shows, in general if the particle masses are assigned correctly the invariant mass of these tracks will be closer to the nominal $K^{* 0}$ mass than in case of a wrong assignment. If both track combinations pass the $K^{* 0}$ mass cut, then the combination with a mass closer to $m_{K^{* 0}}^{\mathrm{PDG}}$ is used. The reconstructed $K^{* 0}$ mass is dominated by the natural width of the $K^{* 0}$ of 50.7 $\mathrm{MeV} \pm 0.6 \mathrm{MeV}$ (see fig. 5.26). The natural width of the $K^{* 0}$ is approximately 10 times bigger than the natural $\phi$ width, so the background when combining a $K^{* 0}$ and a $J / \psi$ candidate to form a $B_{d}^{0}$ is considerably higher. Fig. 5.27 shows that even in the signal sample the $B_{d}^{0}$ signal is almost swamped by the background if only a $J / \psi$ and a $K^{* 0}$ mass cut are applied.

Similar to the $\phi$ reconstruction a $p_{t}$ cut of $1.5 \mathrm{GeV}$ is applied to the $K^{* 0}$ candidates to reduce background (fig. 5.28).

The $J / \psi$ and $K^{* 0}$ candidates are combined to form $B_{d}^{0}$ and a vertex of all four tracks is fitted. The secondary vertex resolutions of $38 \mu \mathrm{m}$ in $x$ and $53 \mu \mathrm{m}$ in $z$ (fig. 5.29) are comparable to those found in the $B_{s}^{0}$ reconstruction.

As before candidates with two identified muon candidates are given preference and for multiple candidates in an event, the one with the smallest $\chi^{2}$ is used. A summary of all cuts used to reconstruct $B_{d}^{0}$ mesons is given in table 5.4. The $\chi^{2}$ distribution of the selected $B_{d}^{0}$ candidates is shown in fig. 5.30.

The invariant mass for candidates passing all cuts is shown in fig. 5.31. The mass resolution of the $B_{d}^{0}$ is $0.037 \mathrm{GeV}$.

For the measurement of the proper decay length a refitted primary vertex as described in section 5.3.5 is used. The proper decay length resolution for the $B_{d}^{0}$ 
candidates is shown in fig. 5.32 .

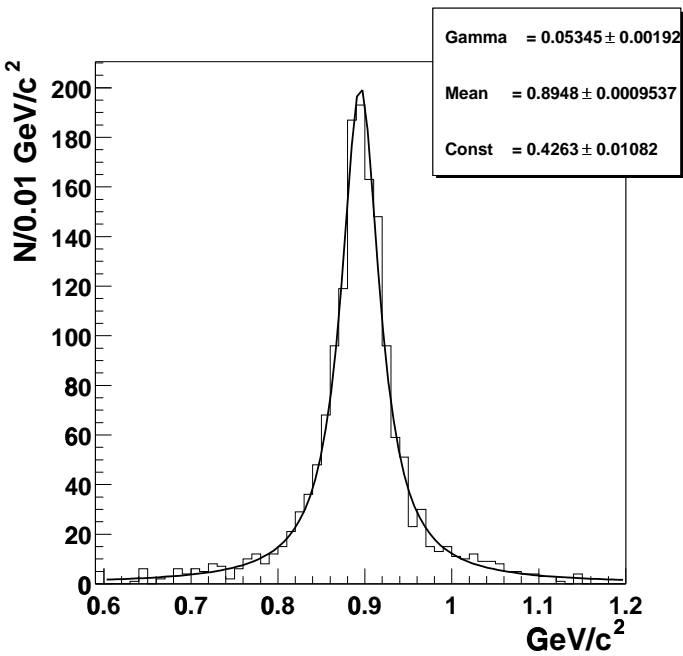

(a) correct assignment of $K$ and $\pi$

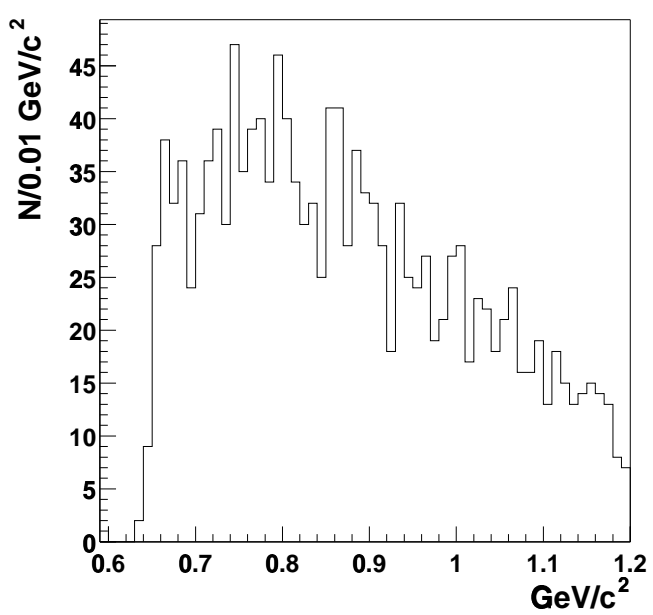

(b) swapped

Figure 5.26: (a) Invariant mass distribution for matched $K^{* 0}$ for the correct assignment of $K$ and $\pi$ masses to their respective tracks and (b) the same candidates with the $K$ and $\pi$ masses swapped. In (a) a Breit-Wigner distribution was fitted.

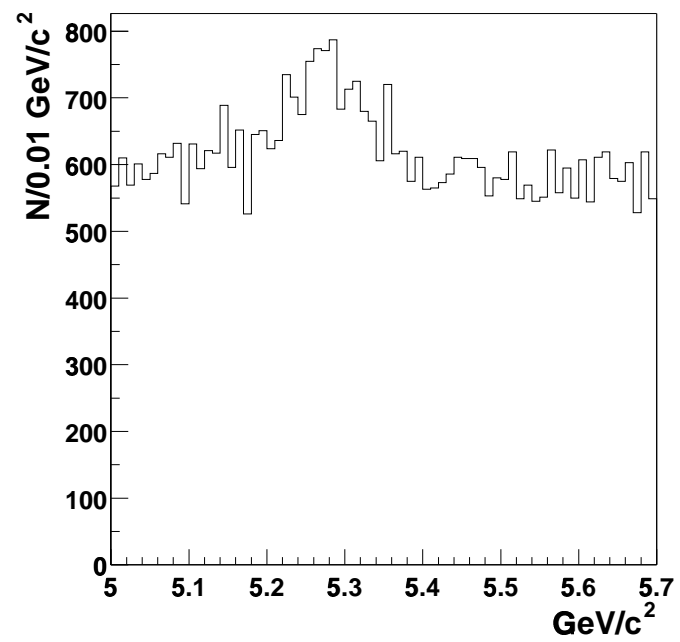

Figure 5.27: Invariant mass of all $B_{d}^{0}$ candidates after applying the $J / \psi$ and $K^{* 0}$ mass cuts for the signal sample only. Unlike in the $B_{s}^{0} \rightarrow J / \psi \phi$ channel the signal is almost swamped by the background. 


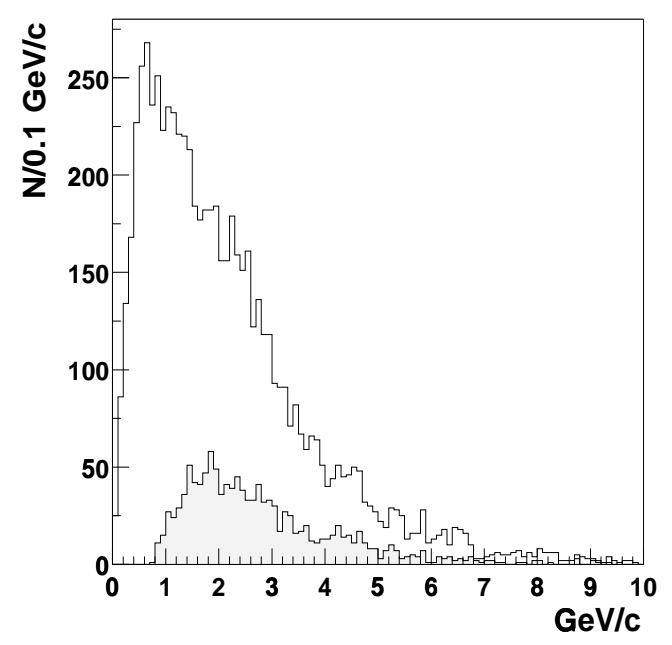

(a)

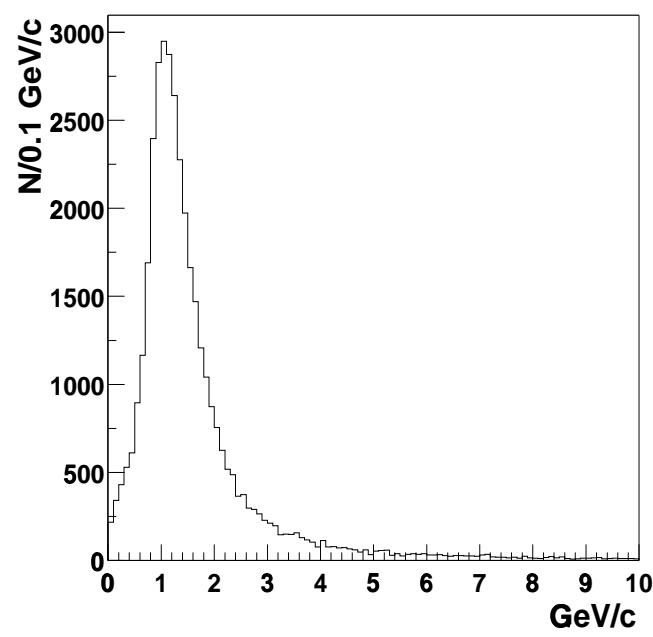

(b)

Figure 5.28: $K^{* 0}$ transverse momentum distribution. Fig. (a) shows the momentum distribution of all simulated $K^{* 0}$ in the signal channel. The shaded histogram is the momentum distribution of all matched $K^{* 0}$ candidates. Fig. (b) shows the $p_{t}$ distribution for background in the $K^{* 0}$ mass window $\left(m_{\mathrm{PDG}} \pm 0.07 \mathrm{GeV}\right)$.

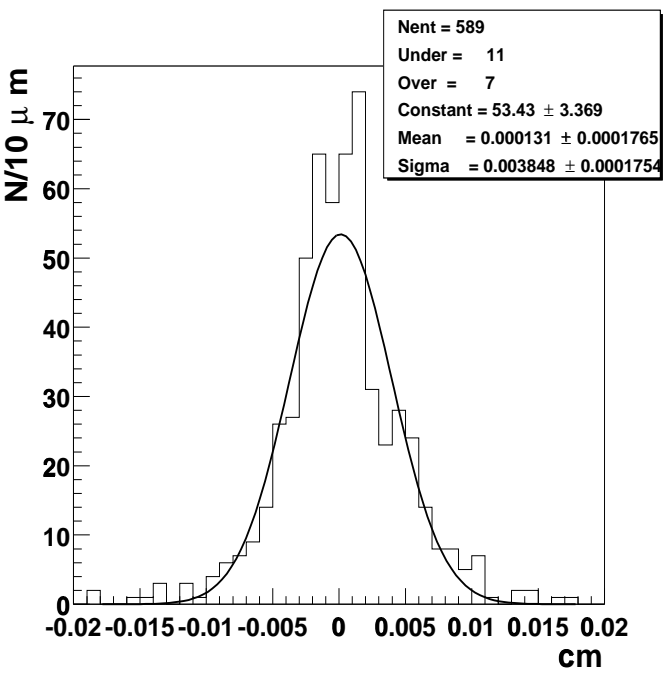

(a) resolution in $x$

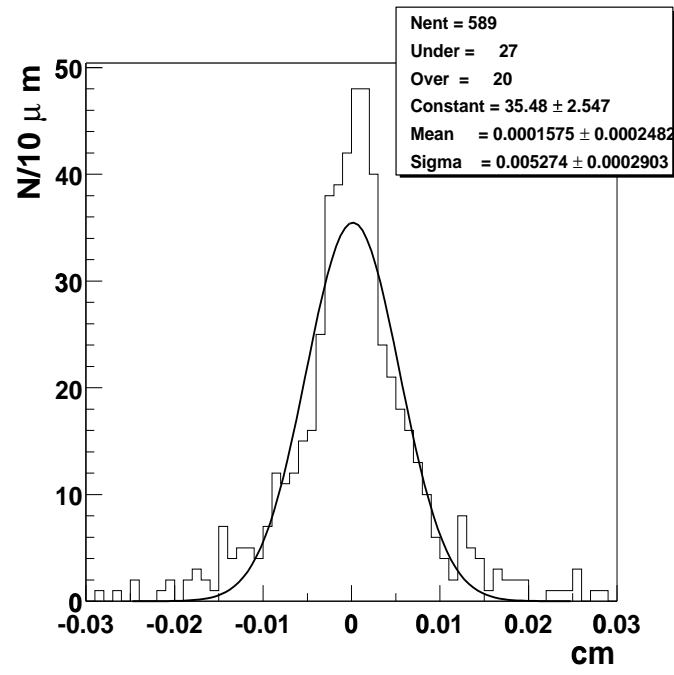

(b) resolution in $z$

Figure 5.29: $B_{d}^{0}$ secondary vertex resolution in $x$ and $z$ with a Gaussian fitted in both cases. 


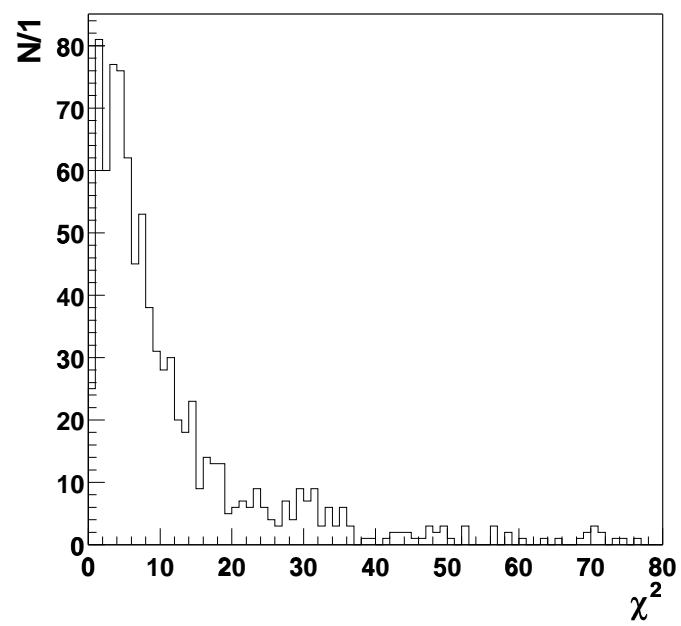

Figure 5.30: $B_{d}^{0} \chi^{2}$ distribution. The four track fit has five degrees of freedom. $B_{d}^{0}$ candidates had to pass all cuts listed in table 5.4 including the $B_{d}^{0}$ mass cut, but not the $B_{d}^{0} \chi^{2}$ cut. If more than one candidate per event passed all cuts, only the candidate with the smallest $\chi^{2}$ was kept.

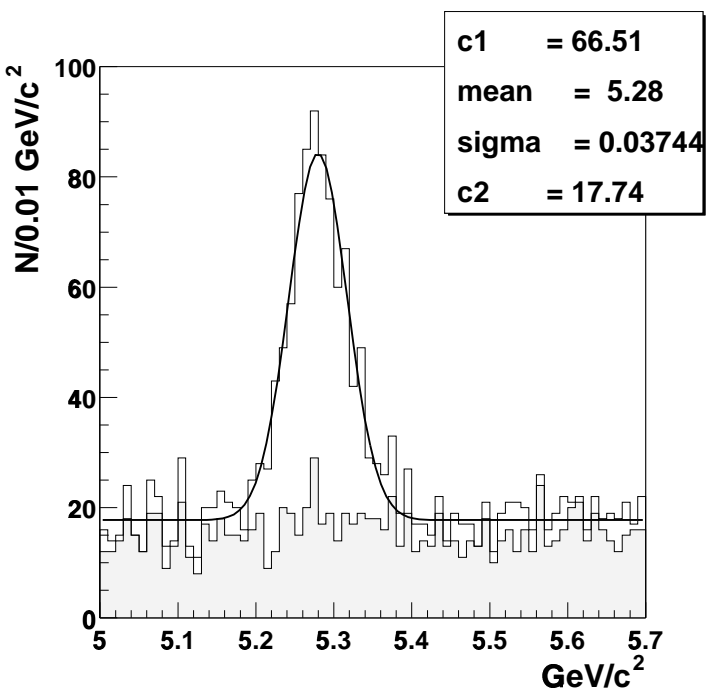

Figure 5.31: Open histogram: Invariant mass of $B_{d}^{0}$ candidates (in signal and background sample) after applying all cuts listed in table 5.4. Shaded histogram: Same for background sample only.

The fitted function is a Gaussian and a constant. 


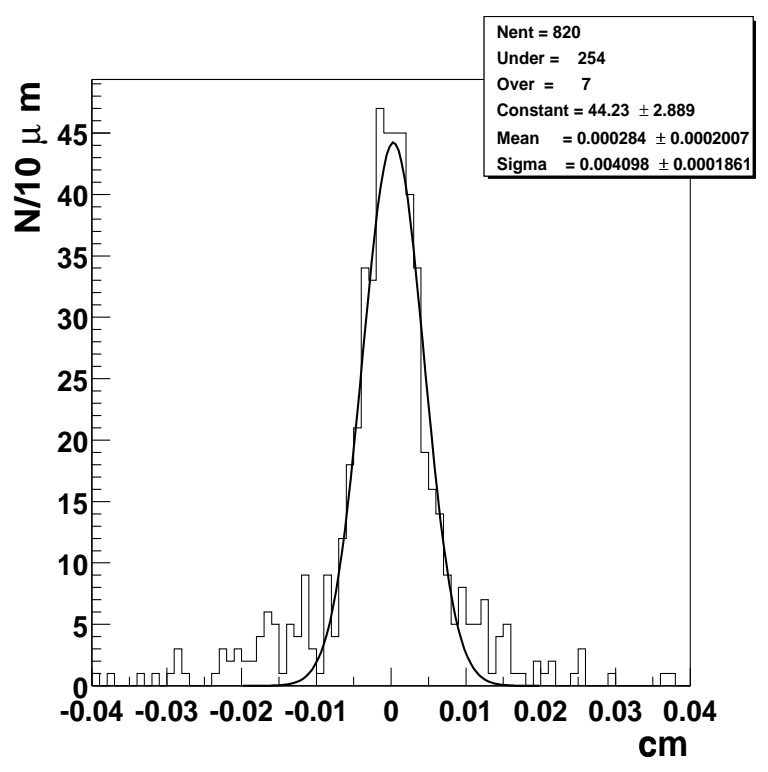

(a)

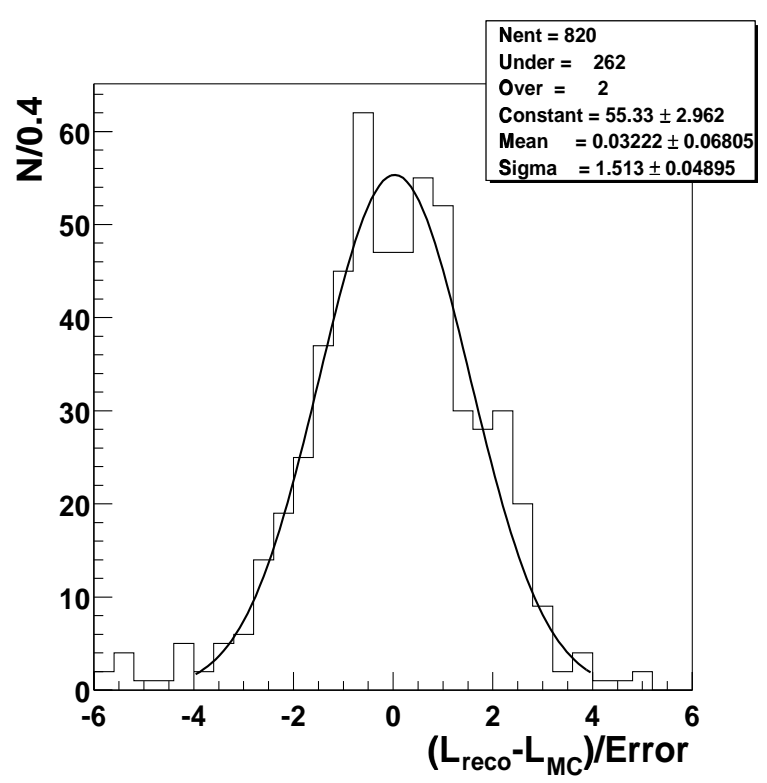

(b)

Figure 5.32: (a) Proper decay length resolution (reconstructed proper decay length-MC proper decay length) for $B_{d}^{0} \rightarrow J / \psi K^{* 0}$. If the event was a background event the MC proper decay length was set to 1000.0 which causes the excess of events in the underflow bin. (b) Difference between the MC proper decay length and the reconstructed proper decay length divided by the error on the proper decay length according to eq. 5.5. 


\begin{tabular}{|l|l|}
\hline cut & reference \\
\hline \hline$\left|m_{J / \psi}-m_{J / \psi}^{\mathrm{PDG}}\right|<0.09 \mathrm{GeV}$ & $2 \sigma$ of $J / \psi$ mass resolution (fig. 5.5$)$ \\
\hline$J / \psi$ fit $\chi^{2}<10.0$ & see fig. 5.9 \\
\hline$\left|m_{K^{* 0}}-m_{K^{* 0}}^{\mathrm{PDG}}\right|<0.07 \mathrm{GeV}$ & see fig. 5.26 \\
\hline $\begin{array}{l}\text { prefer } K^{* 0} \text { candidates with mass closer } \\
\text { to } m_{K^{* 0}}^{\mathrm{PDG}}\end{array}$ & see fig. 5.26 \\
\hline$p_{t}$ of $K^{* 0}$ candidate $>1.5 \mathrm{GeV}$ & see fig. 5.28 \\
\hline $\begin{array}{l}\text { prefer } B_{d}^{0} \text { candidates with two } \\
\text { identified muons }\end{array}$ & see fig. 5.5 \\
\hline$p_{t}$ of $B_{d}^{0}$ candidate $>3.0 \mathrm{GeV}$ & analogous $B_{s}^{0}$ reconstruction \\
\hline$\chi^{2}$ four track fit $<50.0$ & see fig. 5.30 \\
\hline $\begin{array}{l}\text { choose candidate with best } \chi^{2} \text { in four } \\
\text { track fit }\end{array}$ & cf. section 2.8 \\
\hline \hline $\begin{array}{l}\text { distance between primary vertex and } \\
\text { secondary vertex }<2 \mathrm{~cm}\end{array}$ & unphysical \\
\hline \begin{tabular}{l}
$\left|m_{B_{d}^{0}}-m_{B_{d}^{0}}^{\mathrm{PDG}}\right|<0.07 \mathrm{GeV}$ \\
\hline
\end{tabular} & see fig. 5.31 \\
\hline
\end{tabular}

Table 5.4: Summary of cuts used for $B_{d}^{0}$ reconstruction. The last two cuts are only used in the lifetime fit.

\subsubsection{Lifetime}

The higher background in the $B_{d}^{0}$ signal region $\left(\left|m_{B_{d}^{0}}^{\mathrm{reco}}-m_{B_{d}^{0}}^{\mathrm{PDG}}\right|<0.07 \mathrm{GeV}\right)$ requires a more complicated function to model the background contribution than in the case of the $B_{s}^{0}$. The likelihood function for the background now consists of a central Gaussian with two exponential tails. The tail for positive lifetimes contains a number of long-lived particles misidentified as a $B_{d}^{0}$, while negative lifetimes are mostly due to the decay of short-lived particles or random combinations of four tracks. The parametrization of the background as a negative exponential is based on previous analyses [71] and does not represent any physical process. Therefore the fraction of events in both tails can be different. The likelihood function for the background is 
then given by

$$
\begin{aligned}
\mathcal{L}_{\mathrm{bg}}= & \left(1-f_{+}-f_{-}\right) \mathcal{G}\left(L_{i}, \sigma_{i}\right) \\
& + \begin{cases}\lambda_{+}{\frac{f_{+}}{e}}^{-L_{i} / \lambda_{+}} & \text {if } L_{i} \geq 0, \\
\lambda_{-}{\frac{f_{-}}{e}}^{L_{i} / \lambda_{-}} & \text {if } L_{i}<0 .\end{cases}
\end{aligned}
$$

where $f_{-}$and $f_{+}$are the fraction of events in the negative and positive tails. The sidebands are defined as $B_{d}^{0}$ candidates with masses $m_{B}$ fulfilling $0.4 \mathrm{GeV}>\mid m_{B_{d}^{0}}^{\text {reco }}$ $m_{B_{d}^{0}}^{\mathrm{PDG}} \mid>0.07 \mathrm{GeV}$. The likelihood function for the signal is the same as before.

The lifetime distribution for the $B_{d}^{0}$ candidates that pass all cuts given in table 5.4 is shown in fig. 5.33. Fig. 5.34 shows the lifetime distribution of the sidebands.

The fit finds a $B_{d}^{0}$ lifetime of $1.598 \mathrm{ps} \pm 0.080 \mathrm{ps}$. The simulated lifetime was 1.538 ps. The full results of the fit are given in table 5.5. Current lifetime measurements for the $B_{d}^{0}$ yield $1.548 \pm 0.032$ ps. Again, the Monte Carlo measurement was limited by the size of the data sample. With an expected event yield of $\sim 30000$ events in Run II an improvement of the $B^{0}$ lifetime measurement in this channel should be possible.

\begin{tabular}{|l|c|}
\hline$\lambda_{B_{d}^{0}}$ Lifetime of $B_{d}^{0}$ & $1.598 \pm 0.080 \mathrm{ps}$ \\
Error scale factor & $1.60 \pm 0.52$ \\
$\lambda_{+}$Lifetime of positive tail & $0.947 \pm 0.050 \mathrm{ps}$ \\
$f_{+}$Fraction of background events in positive tail & $0.42 \pm 0.02$ \\
$\lambda_{-}$Lifetime of negative tail & $1.424 \pm 0.547 \mathrm{ps}$ \\
$f_{-}$Fraction of background events in negative tail & $0.24 \pm 0.07$ \\
Number of signal events & $600 \pm 32$ \\
Number of background events in signal region & $219 \pm 27$ \\
Number of events in sideband & $1122 \pm 30$ \\
\hline
\end{tabular}

Table 5.5: Results from the unbinned maximum likelihood fit for the $B_{d}^{0}$ lifetime. The simulated lifetime was $1.5388 \mathrm{ps}(461 \mu \mathrm{m})$. 


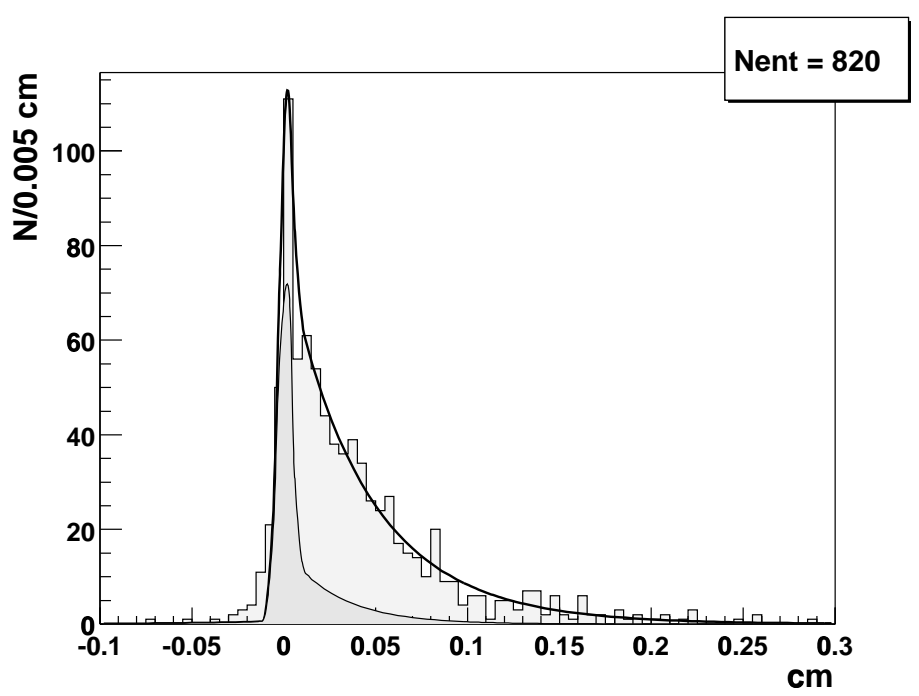

Figure 5.33: Proper decay length distribution for events in the signal region. The fit was an unbinned likelihood fit, so the fit function is plotted with a fixed value for the resolution, while during the fit the individual error for each entry is used. The upper curve is the full likelihood function (signal and background), the lower curve is the background contribution in the signal region.

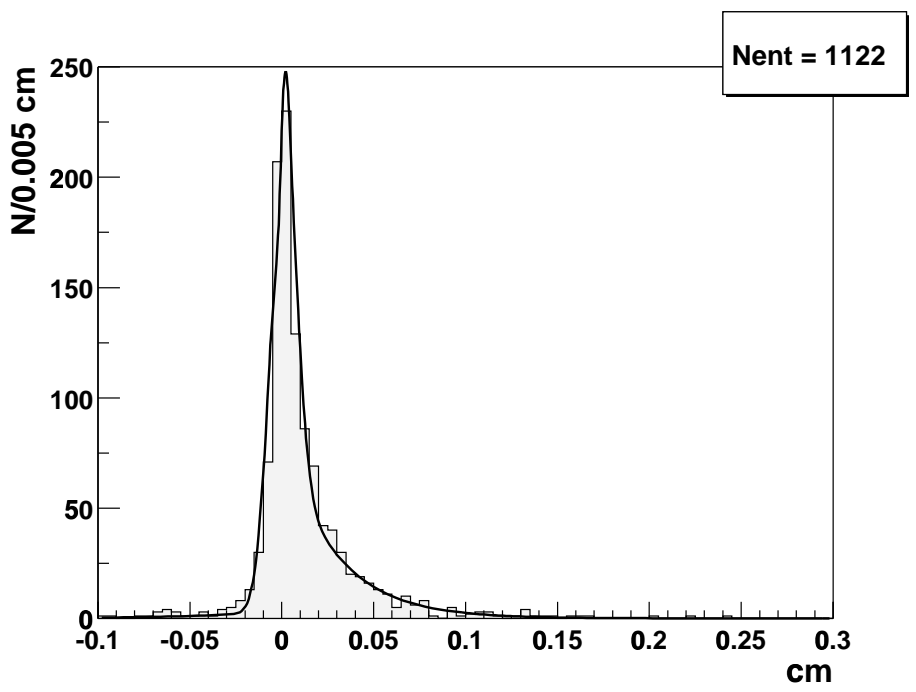

Figure 5.34: Proper decay length distribution in sideband region. 


\subsection{Cross-talk}

In a detector without particle identification interpreting a $K$ as a $\pi$ or vice-versa can lead to confusing $\phi \rightarrow K^{+} K^{-}$with $K^{* 0} \rightarrow K^{+} \pi^{-}$decays. This can lead to confusion between the two signal channels $B_{s}^{0} \rightarrow J / \psi \phi$ and $B_{d}^{0} \rightarrow J / \psi K^{* 0}$.

In fig. 5.35 the mass distributions for an equal number of $B_{s}^{0} \rightarrow J / \psi \phi$ and $B_{d}^{0} \rightarrow J / \psi K^{* 0}$ events reconstructed as $B_{s}^{0}$ and $B_{d}^{0}$ are shown.

The probability of a $B_{s}^{0}$ to be confused with a $B_{d}^{0}$ is $1.5 \%$ while for a $B_{d}^{0}$ to be reconstructed as a $B_{s}^{0}$ it is only $0.16 \%$. But while per meson it is more likely for a $B_{s}^{0}$ to fake a $B_{d}^{0}$ than vice-versa, there are considerably less $B_{s}^{0}$ than $B_{d}^{0}$ produced at the Tevatron $\left(B_{s}^{0}: B_{d}^{0} \approx 1: 3\right)$. Also the branching ratio for the full decay chain of $B_{s}^{0} \rightarrow J / \psi \phi\left(2.7 \times 10^{-5}\right)$ is approximately half the $B_{d}^{0} \rightarrow J / \psi K^{* 0}$ branching ratio of $5.8 \times 10^{-5}$. Taking into account the reconstruction efficiency for $B_{s}^{0} \rightarrow J / \psi \phi$ of $10 \%$ and an $8.5 \%$ efficiency for $B_{d}^{0} \rightarrow J / \psi K^{* 0}$ the expected contamination through cross-talk is $9.0 \%$ in the $B_{s}^{0}$ signal and $2.8 \%$ in the $B_{d}^{0}$ signal.

\subsection{Event yields}

The error on the lifetime measurements will depend on the number of signal events $\mathrm{D} \varnothing$ will be able to reconstruct. In this section I give an estimate for the expected event yields in both signal channels for an integrated luminosity of $2 \mathrm{fb}^{-1}$.

As mentioned in section 2.9 there is a discrepancy between the predicted and the measured $b \bar{b}$ cross-section. For calculations here the number of $b \bar{b}$ pairs used is $2 \times 10^{11}$.

The main trigger for both signal channels will be a di-muon trigger. As no full trigger simulation was available, the trigger efficiency quoted here is an efficiency for the Level 1 trigger. It was calculated from a simulation for $B_{d}^{0} \rightarrow J / \psi K_{s}^{0}$, a decay which relies on the same triggers as the signal channels. The sample contained 1000 events, with a minimum $p_{t}$ of $1.5 \mathrm{GeV}$ and $|\eta|<1.6$ for both muons [11]. No studies had been done for the Level 2 and 3 triggers. Generally lower luminosities (as currently seen at the Tevatron) favour b-physics at trigger level. 


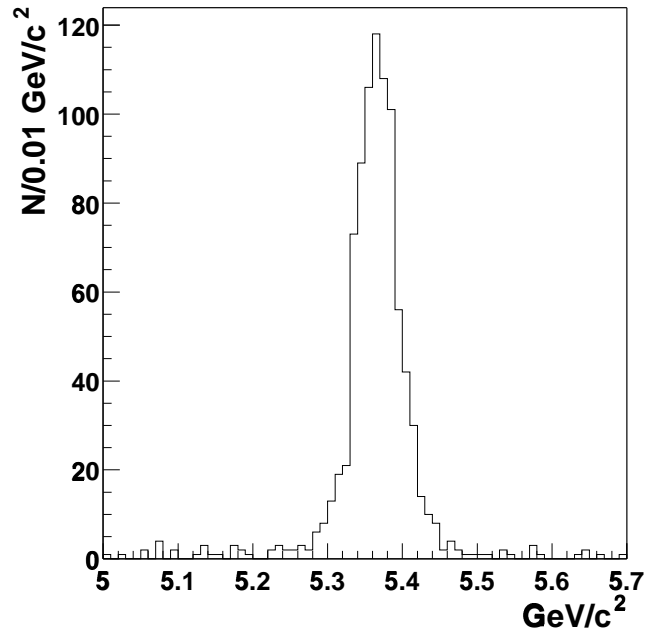

(a) $B_{s}^{0}$ reconstructed as $B_{s}^{0}$.

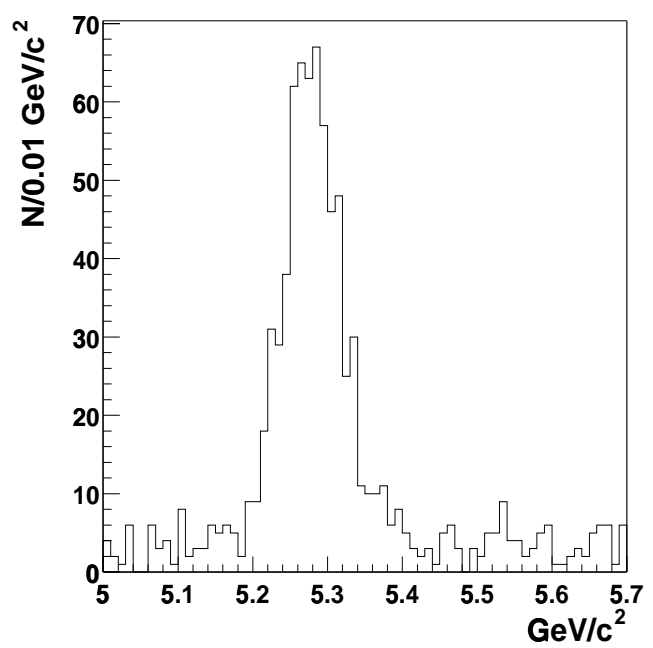

(c) $B_{d}^{0}$ reconstructed as $B_{d}^{0}$.

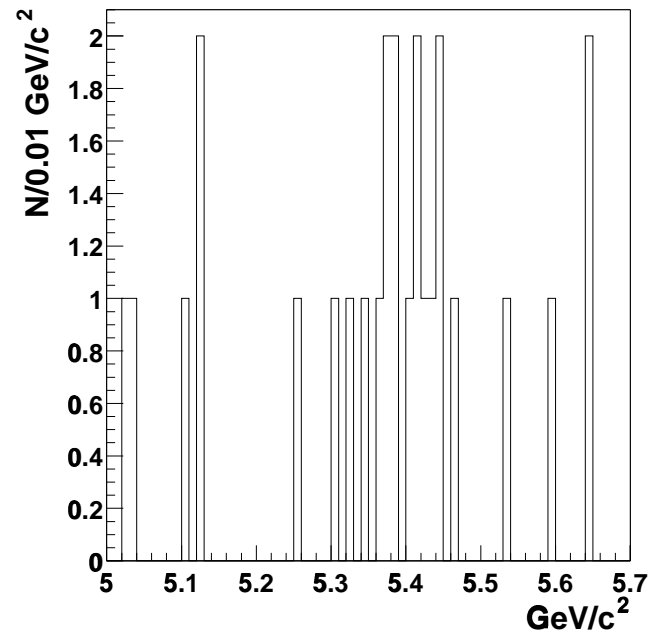

(b) $B_{d}^{0}$ reconstructed as $B_{s}^{0}$.

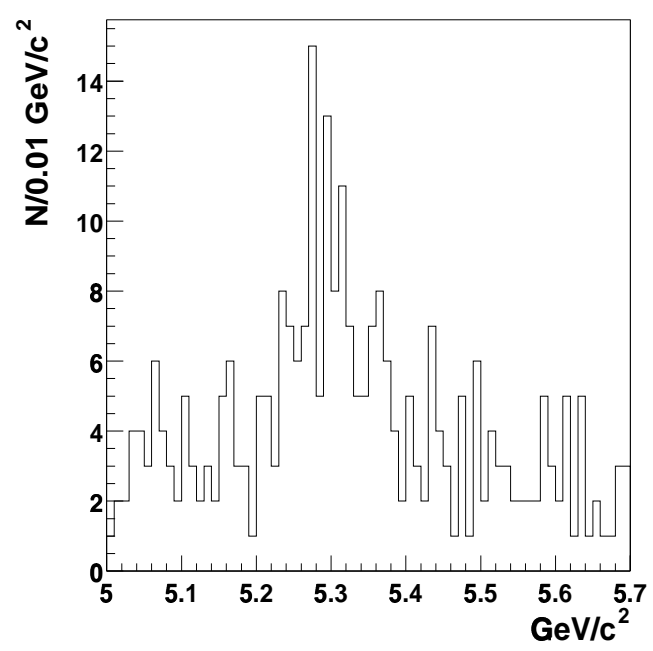

(d) $B_{s}^{0}$ reconstructed as $B_{d}^{0}$.

Figure 5.35: Cross-talk: Reconstructing $B_{s}^{0} \rightarrow J / \psi \phi$ as $B_{d}^{0} \rightarrow J / \psi K^{* 0}$ and vice-versa. Fig. (b) contains 11 entries in the $B_{s}^{0}$ mass range. Fig. (d) contains 105 entries in the $B_{d}^{0}$ mass range.

It is also assumed that events which did not pass the generator level cuts will not be reconstructed. As these cuts $\left(p_{t}^{\mu}>1.5 \mathrm{GeV},\left|\eta_{\mu}\right|<2\right)$ mostly represent the detector acceptance, it is indeed unlikely that any of these events could be seen in the detector. 
All input factors are summarized in table 5.6. The resulting event yields are 6000 fully reconstructed events for $B_{s}^{0} \rightarrow J / \psi \phi$ and 30000 for $B_{d}^{0} \rightarrow J / \psi K^{* 0}$.

\begin{tabular}{|l|c|}
\hline $\begin{array}{l}\text { integrated luminosity Run II } \\
\sigma(b \bar{b})\end{array}$ & $2 \mathrm{fb}^{-1}$ \\
\hline total number of $b \bar{b}$ pairs & $(2-3) \times 158 \mu \mathrm{b}$ \\
fragmentation fraction $f_{s}$ & $0.160 \pm 0.044$ \\
fragmentation fraction $f_{d}$ & $0.375 \pm 0.023$ \\
\hline Branching fraction $B_{s}^{0} \rightarrow J / \psi \phi$ & $(9.3 \pm 3.3) \times 10^{-4}$ \\
Branching fraction $B_{d}^{0} \rightarrow J / \psi K^{* 0}$ & $(1.50 \pm 0.17) \times 10^{-3}$ \\
Branching fraction $J / \psi \rightarrow \mu^{+} \mu^{-}$ & $(5.88 \pm 0.10) \%$ \\
Branching fraction $\phi \rightarrow K^{+} K^{-}$ & $(49.2 \pm 0.7) \%$ \\
Branching fraction $K^{0 *} \rightarrow K^{+} \pi^{-}$ & $06 \%\left(K^{* 0} \rightarrow K \pi \sim 100 \%\right)$ \\
\hline Trigger efficiency & $12 \%$ \\
\hline$B_{s}^{0}$ Percentage of events that pass cuts at generator level & 0.1 \\
$B_{s}^{0}$ Reconstruction efficiency (of sample with cuts) & $14 \%$ \\
$B_{d}^{0}$ Percentage of events that pass cuts at generator level & 0.085 \\
$B_{d}^{0}$ Reconstruction efficiency (of sample with cuts) & 6000 \\
\hline \hline Event yield $B_{s}^{0} \rightarrow J / \psi \phi$ & 30000 \\
Event yield $B_{d}^{0} \rightarrow J / \psi K^{0 *}$ & \\
\hline
\end{tabular}

Table 5.6: Event yields for Run II a. All branching fractions are taken from [3], the fragmentation fractions from [30] and the cross-section from [11]. Note that there is a large error $(\sim 35 \%)$ on the $B_{s}^{0}$ branching fraction. The trigger efficiency is based on studies for $B_{d}^{0} \rightarrow J / \psi K_{S}^{0}$ which relies on the same di-muon trigger as the signal channels [11]. The reconstruction efficiency used is based on the results of this thesis.

\subsection{Angular distributions}

\subsubsection{Generators for $b$-physics}

When I started looking at the angular distributions in the decay $B_{s}^{0} \rightarrow J / \psi \phi$ (with $J / \psi \rightarrow \mu^{+} \mu^{-}$and $\phi \rightarrow K^{+} K^{-}$) I found that they were simulated incorrectly by the 
QQ version available at FNAL. Taking into account that CLEO stopped supporting QQ, I looked into using the BABAR event generator EvtGen [62] instead. EvtGen is available as a public release or on request directly from the BABAR repository. As a recent development it is, like the vast majority of the $\mathrm{D} \emptyset$ software, written in $\mathrm{C}++$ while $\mathrm{QQ}$ still uses Fortran. The software structures at BABAR and $\mathrm{D} \varnothing$ are very similar, so the conversion of EvtGen into a $D \emptyset$ package was straightforward, with only minor adjustments to account for the stricter compiler options at DØ. A major difficulty arose however from the fact that EvtGen uses JetSet [59] and PHOTOS [72] for fragmentation and final state radiation in some of its decays. Based on its own decay table EvtGen creates a new limited decay table for JetSet which replaces the default JetSet decay table. This is done during run-time to reflect any changes made to the EvtGen decay table. The fact that EvtGen was developed using JetSet 7.4 while D $\varnothing$ uses PYTHIA 6.155 [73] (merged with JetSet) required major modifications in the code that generates the modified decay table for JetSet to conform with the more detailed standard set by the new PYTHIA. It also required an update of the particle codes in the EvtGen decay table. Other modifications included changing real type variables to double precision in all Fortran code and renaming a number of common blocks to conform with the new PYTHIA standards. A detailed overview of the porting of EvtGen to D $\varnothing$ can be found in [74].

EvtGen originally only decayed $B_{d}^{0}$ mesons. A decay table including $B_{s}^{0}$ mesons was developed by CDF [75] and is now also used at $\mathrm{D} \varnothing$.

When I stopped active code-development in order to finish this thesis EvtGen ran as a stand-alone executable on Linux and IRIX platforms and initial tests (see figs. 5.36 and 5.37) looked promising. Fig. 5.36 shows the (wrong) transversity angle distribution as simulated by QQ, while fig. 5.37 shows that the distributions for the two $C P$-eigenstates are correctly simulated by EvtGen. EvtGen has now been fully integrated into the $\mathrm{D} \varnothing$ framework [76] and will replace QQ as the default event generator in the near future. 


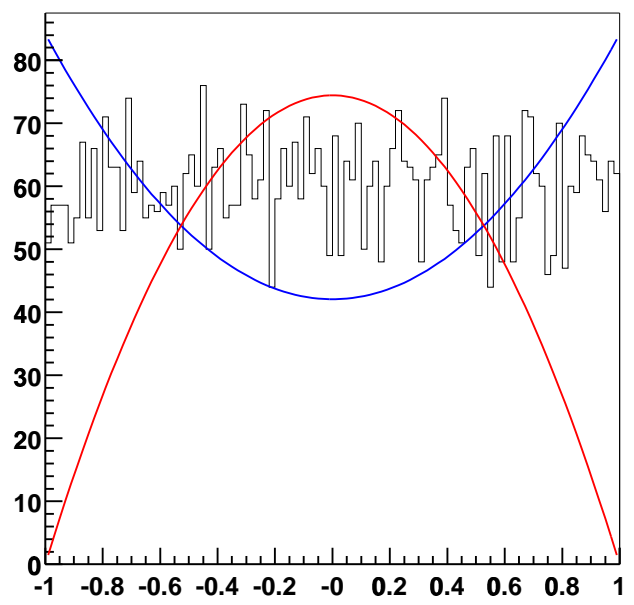

Figure 5.36: Transversity angle of one of the $C P$-eigenstates in $B_{s}^{0} \rightarrow J / \psi \phi$ as simulated by QQ. The distribution is flat. The curves represent the expected distributions for $\mathrm{CP}$ even $\left(1+\cos ^{2} \theta\right)$ and $\mathrm{CP}$ odd $\left(1-\cos ^{2} \theta\right)$ decays.

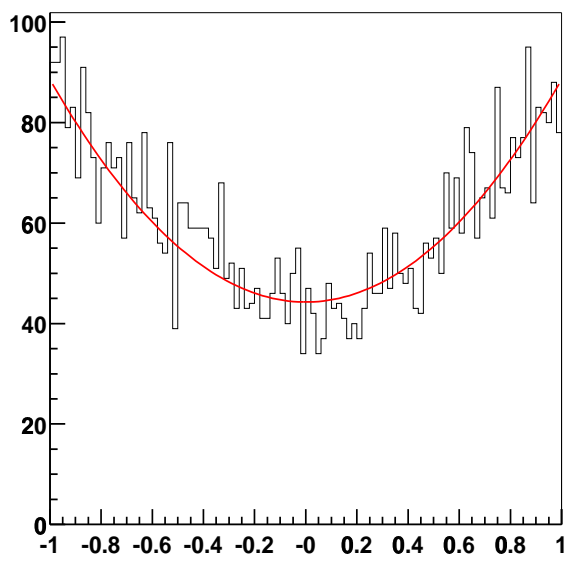

(a) even

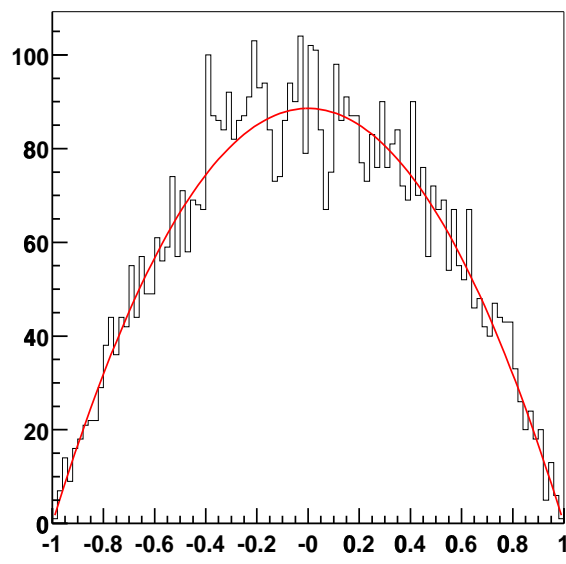

(b) odd

Figure 5.37: Transversity angle for $\mathrm{CP}$ even and odd $B_{s}^{0} \rightarrow J / \psi \phi$ events in EvtGen fitted with the appropriate distributions (see section 2.7). 


\subsubsection{Shaping angular distributions}

To disentangle physics effects from detector contributions when measuring an angular distribution, the influence of the reconstruction on the shape of the distribution needs to be known to a good extent. For example the kinematic cuts $\left(p_{t}^{\mu}>1.5\right)$ will introduce a parabolic shape to an otherwise flat distribution (see fig. 5.38).

The more difficult problem is the shaping introduced by more subtle detector effects, like dependence of the reconstruction efficiencies on the momentum of the particle, making it often impossible to reconstruct low-momentum particles. Fig. 5.39 shows the generated and the reconstructed $\cos \theta_{t}$ spectrum for the $B_{s}^{0} \rightarrow J / \psi \phi$ sample with an additional lifetime cut of $L>50 \mu \mathrm{m}$. A lifetime cut will reduce the background without distorting the transversity angle distribution. The reconstructed spectrum does not reproduce the generated spectrum completely, but there are also no significant differences (e.g. an asymmetric distribution). While this cannot replace a full analysis, it indicates that $\mathrm{D} \varnothing$ should be able to investigate the different $C P$-eigenstates in $B_{s}^{0} \rightarrow J / \psi \phi$.

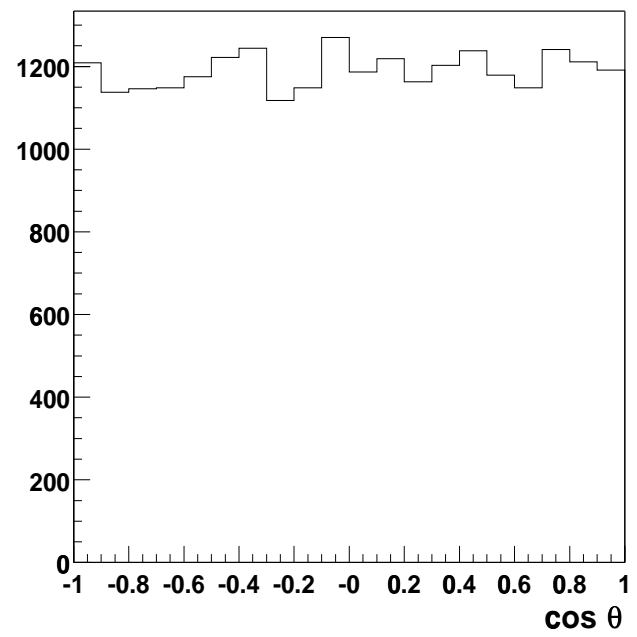

(a)

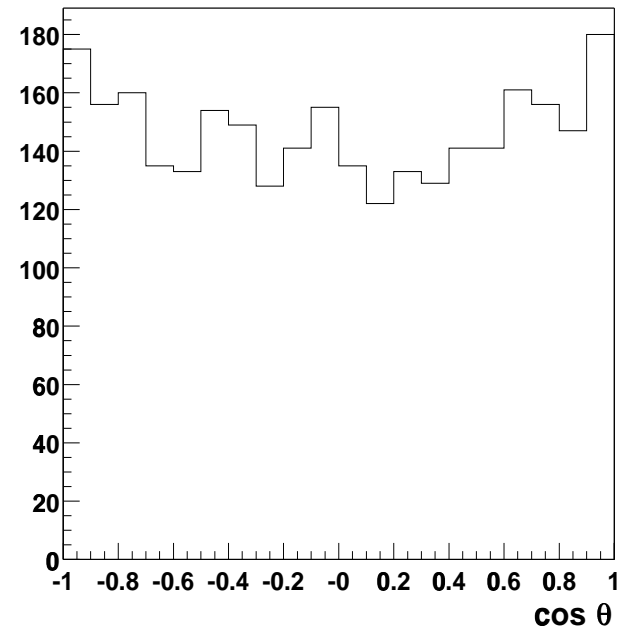

(b)

Figure 5.38: A flat transversity angle distribution at generator level. Fig. (a) shows the generated distribution (by QQ) without any cuts. Fig. (b) shows the same sample when requiring that the muons from the $J / \psi$ have a transverse momentum of at least $1.5 \mathrm{GeV}$. 


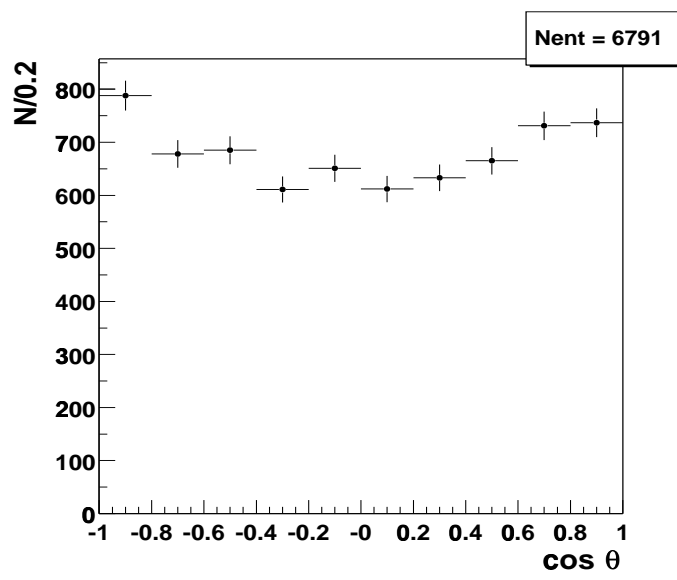

(a) $\mathrm{MC}$

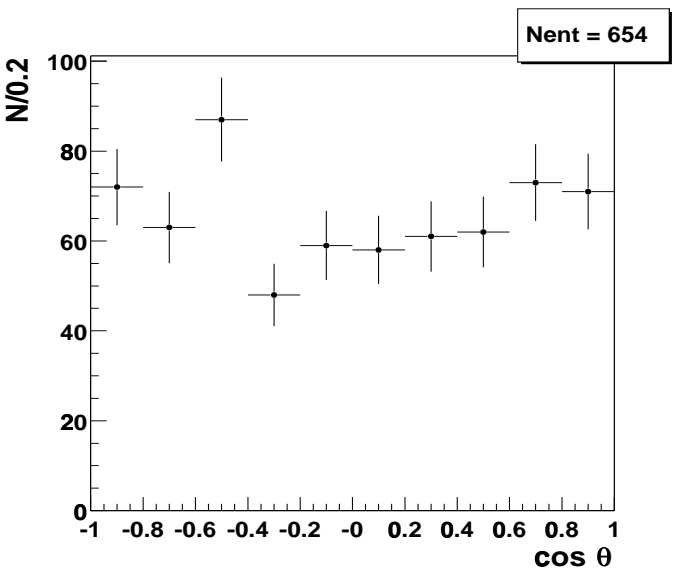

(b) reconstructed

Figure 5.39: Transversity angle distribution in the $B_{s}^{0} \rightarrow J / \psi \phi$ signal sample. Fig. (a) shows the generated distribution, (b) shows the reconstructed distribution for $B_{s}^{0}$ candidates passing all cuts listed in table 5.2 and an additional cut of $L>50 \mu \mathrm{m}$.

\section{$5.8 J / \psi$ reconstruction using real data}

The first major set of data comprising 5.8 million reconstructed events was taken between August and October 2001. During this time, the fibre tracker was not operational, so the muons used were reconstructed by the muon system alone. There is no match between tracks found in the Silicon Microstrip Tracker and those in the muon system, as only tracks with a low transverse momentum $\left(p_{t}<2.5 \mathrm{GeV}\right)$ were reconstructed in the SMT, while the muon reconstruction required a minimum transverse momentum of $3 \mathrm{GeV}$.

The complete data set contained 47389 events with two or more muon candidates. To ensure a minimum momentum resolution, only muon candidates with hits in all three layers of the muon system were used. This left 3060 events, which still included a number of tracks without stereo information. After excluding these muon candidates, fig. 5.40 shows the invariant mass distributions for muon pairs with opposite and same signs.

The background can be reduced by requiring that both muon tracks originate within $3 \mathrm{~cm}$ of the primary vertex. This mainly reduces the random combinations which 
result in the peak at low masses. The peak at $m_{\mu \mu}>6.0 \mathrm{GeV}$ is due to combinations of muons from different quarks and cosmics which are reconstructed as opposite sign muon pairs as there is no matching to global tracks [77].

Fig. 5.41 shows the transverse momentum vs. the invariant mass of the muon pairs. Due to the minimum $p_{t}$ of the muon candidates enforced by the reconstruction there are no $\mu \mu$-combinations with low invariant masses and low momenta. A required minimum transverse momentum of $5 \mathrm{GeV}$ for the $\mu \mu$-combinations will reduce the background further, without diminishing the signal. The $\eta$ distribution in fig. 5.42 shows an excess of candidates in the range $1<|\eta|<2$ for both types (same sign, opposite sign) of muon pairs. This was due to problems in the central muon chambers $(|\eta|<1)$, while the forward muon chambers were working as planned. To reduce background only muon pairs with $\eta<2.1$ were used.

Fig. 5.43 shows the invariant mass distribution after all cuts. Fitted to it was a Gaussian for the signal and a straight line for the background. The signal peak contains $610 \mathrm{~J} / \psi$. It peaks at $3.54 \mathrm{GeV}$ with a $\sigma$ of $0.97 \mathrm{GeV}$. The bias towards a higher mass than the nominal $J / \psi$ mass of $3.09 \mathrm{GeV}$ is expected to disappear when the full reconstruction is used. The width of the peak is within $20 \%$ of the MC expectation of $0.81 \pm 0.08 \mathrm{GeV}[67]$.

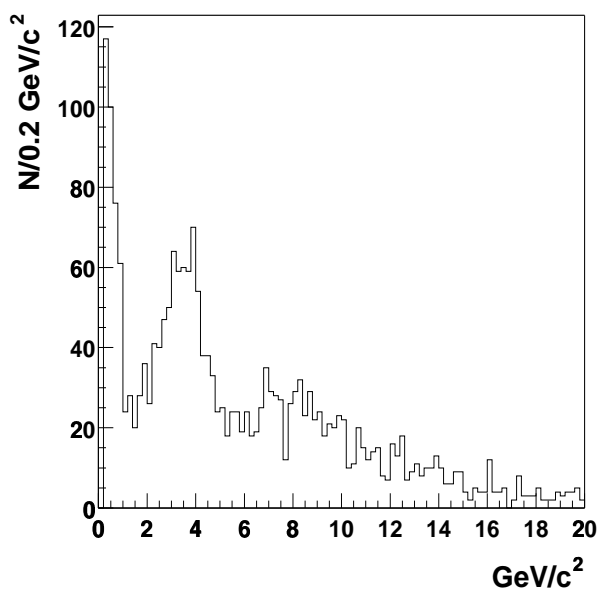

(a) opposite charges

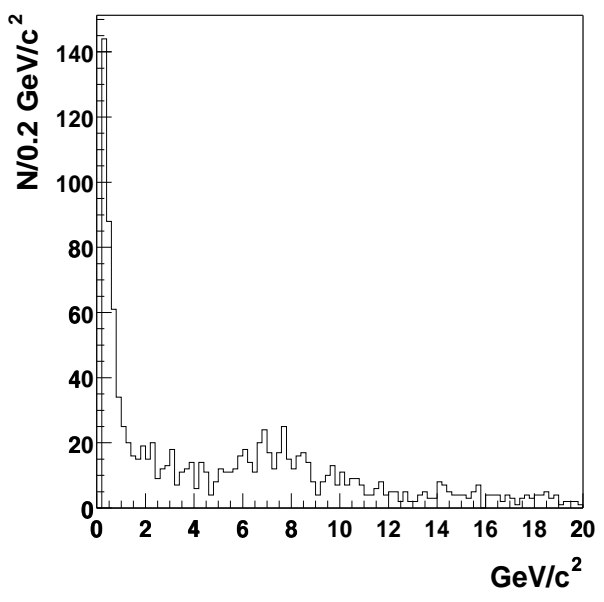

(b) same charges

Figure 5.40: Invariant mass distribution for muon pairs using minimal cuts only. 


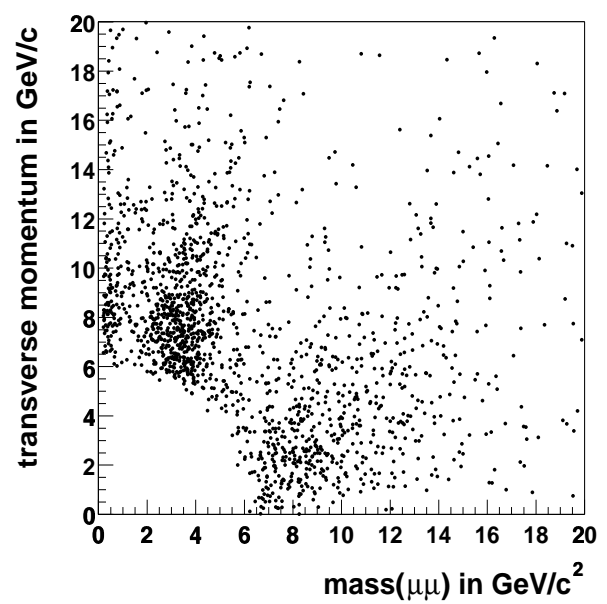

(a) combinations of opposite sign tracks

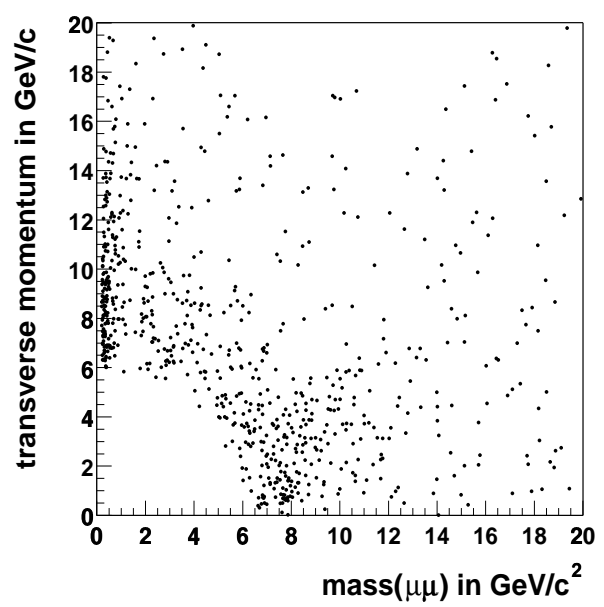

(b) combinations of same sign tracks

Figure 5.41: Transverse momentum vs. invariant mass of muon pairs. Only events with at least two muon candidates with hits in all three layers of the muon system were used.

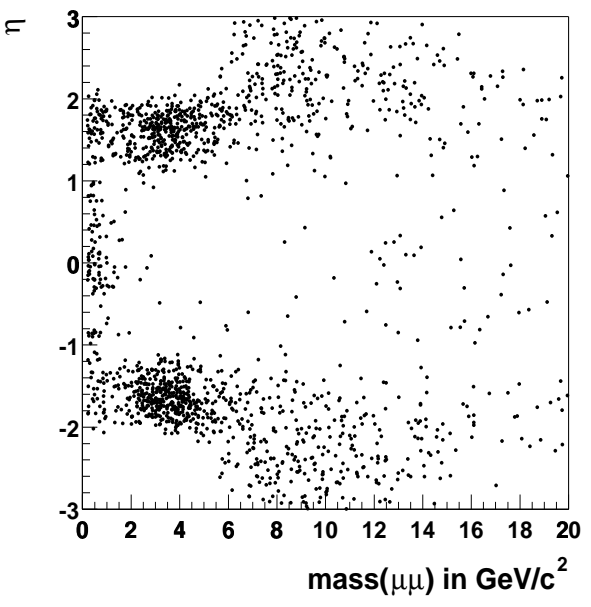

(a) opposite charges

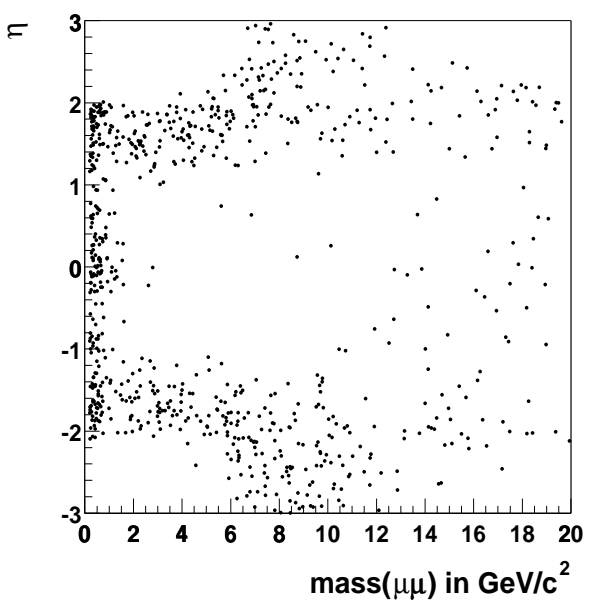

(b) same charges

Figure 5.42: Pseudo-rapidity $\eta$ vs. invariant mass of muon pairs. Only events with at least two muon candidates with hits in all three layers of the muon system were used. 


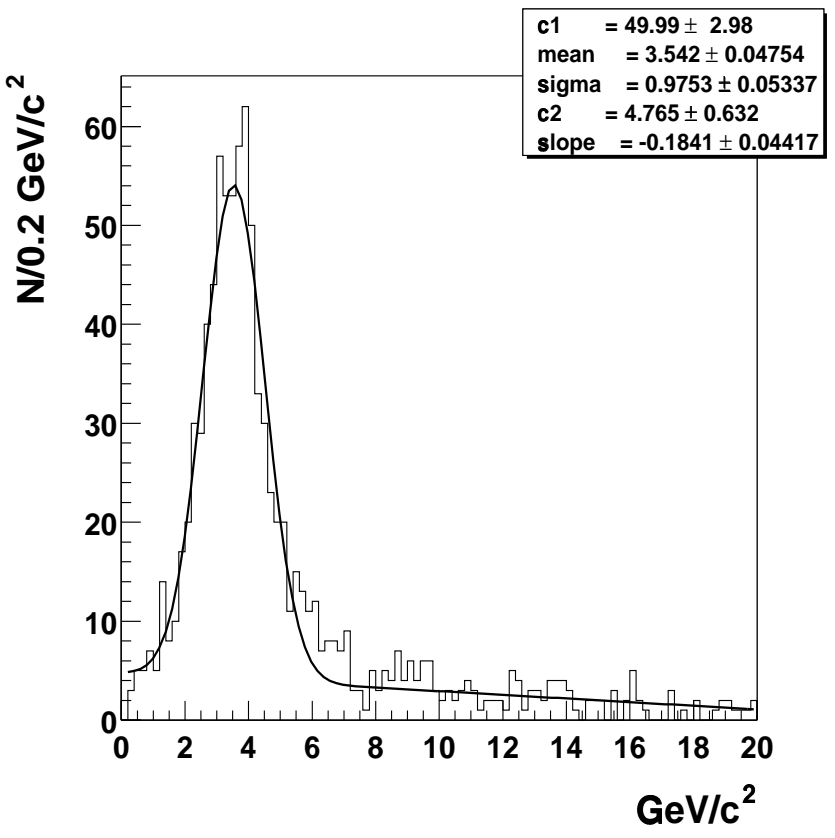

(a) opposite charges

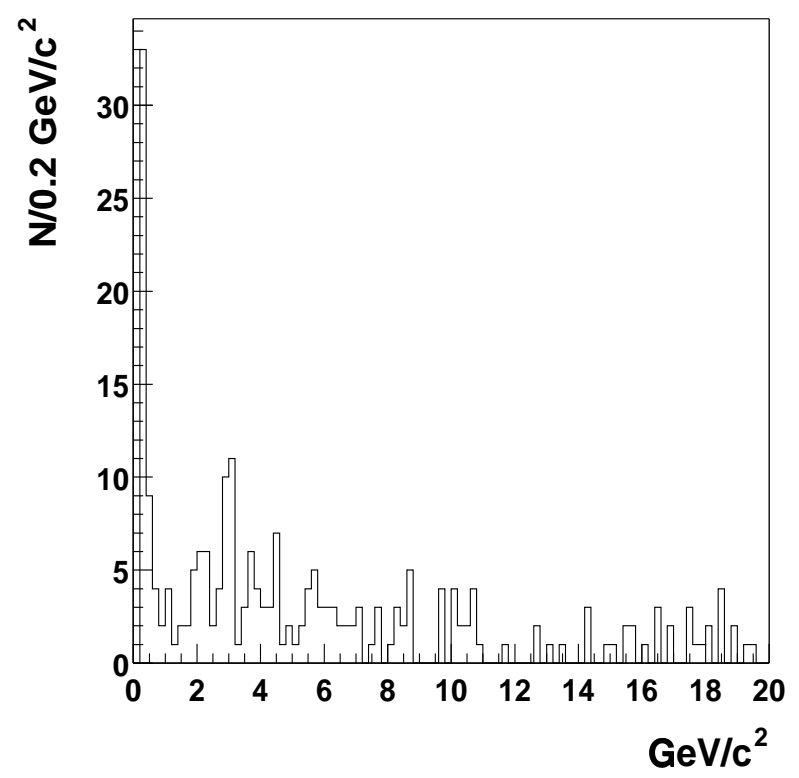

(b) same charges

Figure 5.43: Invariant mass distribution for muon pairs after applying all cuts. 


\section{Chapter 6}

\section{Technology transfer}

\subsection{Introduction}

Traditionally High Energy Physics (HEP) is very research orientated and few attempts are made to exploit its potential for commercialisation.

The recently completed analysis of the human genome emphasizes the need for new methodologies in high throughput macromolecular separation and analysis. Possible contributions of HEP in this area include pattern recognition, simulations, signal processing, instrumentation (e.g. photon detectors), electronics and computing.

The HEP group at Imperial College has a number of projects aimed at the transfer of HEP technologies into other areas, emphasizing the use of HEP technologies in industrial applications.

The $\mu$ DiaGene project which is described in this chapter concerns the development of a high throughput DNA sequencing chip. As part of my work for $\mu$ DiaGene I developed MEDUSA, a simulation package to model the movement and detection of DNA bands on a UV transparent chip.

The chapter begins with a short introduction to DNA sequencing, followed by an overview of the $\mu$ DiaGene project. The last section of this chapter describes MEDUSA. 

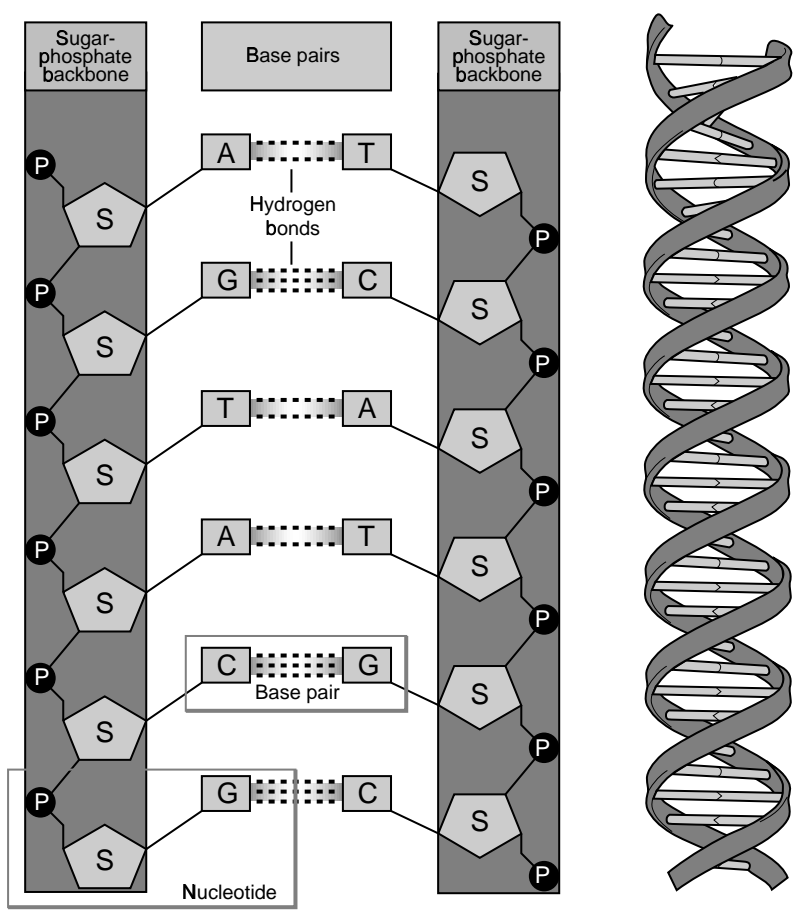

Figure 6.1: DNA structure (Picture taken from [79])

\subsection{DNA sequencing}

\subsubsection{The DNA helix}

Deoxyribonucleic acid (DNA) forms the genetic material in all cells. It forms a righthanded double helix consisting of a sugar-phosphate backbone with four different types of bases attached to it (fig. 6.1). Its structure was first described in 1953 by Watson and Crick [78]. The bases are located inside the helix, held together by hydrogen bonds. The four bases are: Thymine(T), Adenine(A), Cytosine(C) and Guanine $(\mathrm{G})$. Thymine always couples to Adenine and Cytosine to Guanine (fig. $6.2)$.

\subsubsection{Traditional DNA sequencing}

In order to understand the genetic code described in DNA it is essential to determine the sequence in which the four bases appear. This is done by using electrophoresis 

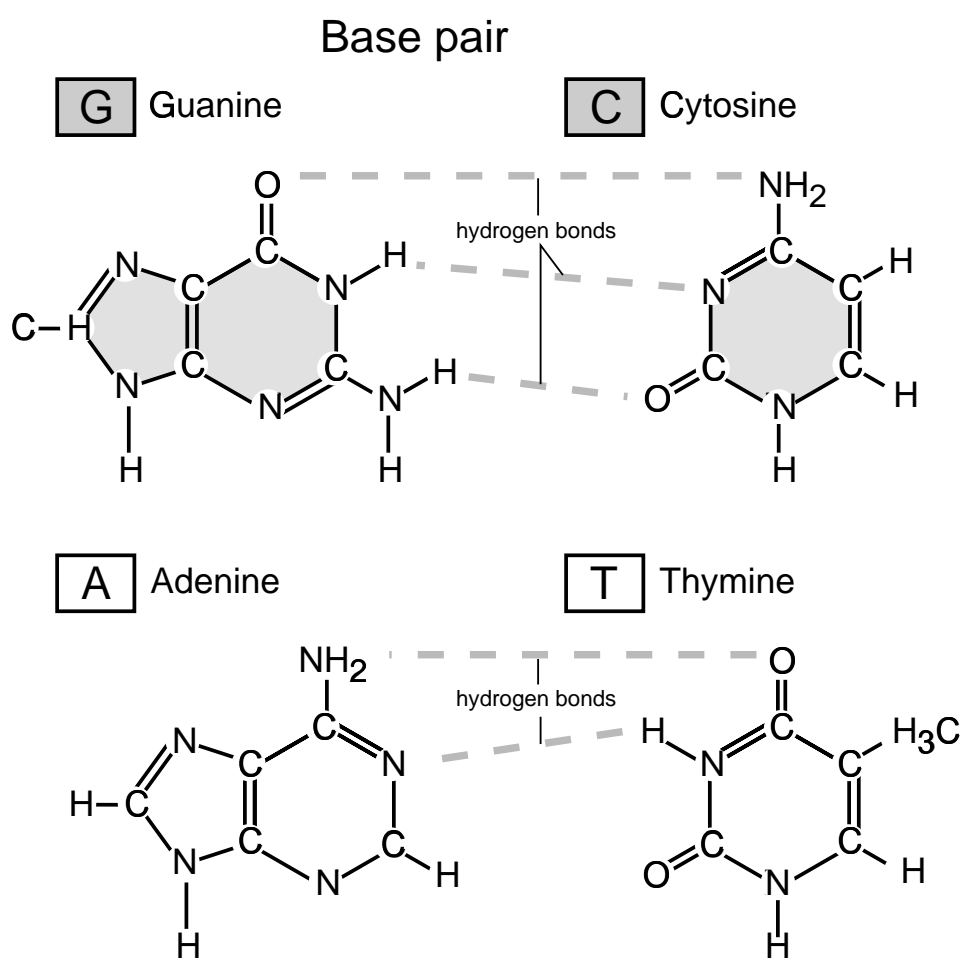

Figure 6.2: The four bases (Picture taken from [79]).

in sequencing gels. Sequencing gels are designed to $\underset{C}{\text { fractionate negatively charged }}$

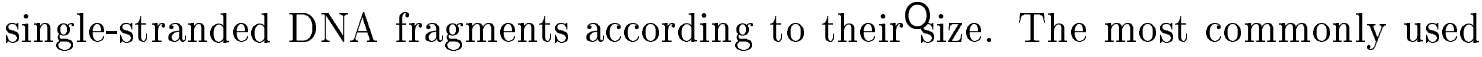
gels are polyacrylamide for small and agarose for large fragments up to 20000 basepairs. Agarose is a polysaccharide obtained from seaweed. Agarose gels have the advantage of being non-toxic, while polyacrylamide can contain free acrylamide, a neurotoxin. As oxygen inhibits the polymerization process, the preparation of polyacrylamide gels is significantly more complicated than of agarose gels, which are obtained by melting the agarose in the desired buffer.

Electrophoresis is based on the migration of ions in an electric field. The charge to mass ratio of DNA fragments is constant, i.e. the electrophoretic mobility of DNA in free solution is independent of its molecular weight. The separation of fragments of different lengths in sequencing gels is a result of the movement of the fragments through the pores of the gel. Small fragments suffer less resistance to their movement than larger ones and are therefore faster. Once the size of the fragments exceeds 


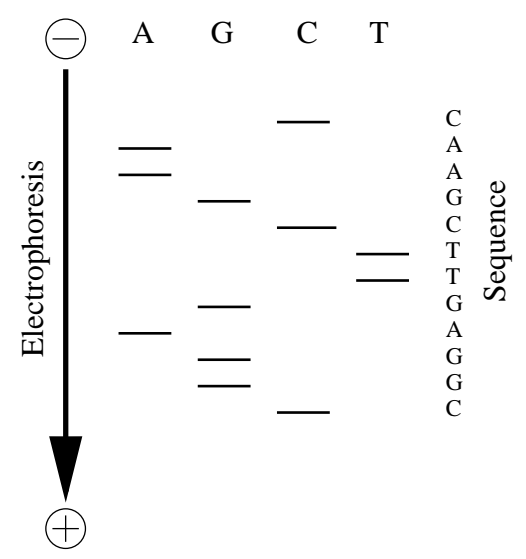

Figure 6.3: Traditional DNA sequencing (schematic).

the pore size the mobility becomes size-independent and no further separation is possible.

All sequencing methods require the separation of the helix into two strands. This can be done chemically or by using heat. In order to retrieve a sequence it is necessary to obtain fragments of the original DNA or its copies which end in a predetermined type of base-pair. The two most common ways to achieve this are outlined below.

\section{The Sanger or chain termination method}

The Sanger [80] method requires a number of reagents:

- Deoxynucleoside triphosphate (dNTP): A base N (N=A,G,T,C) and its part of the sugar-phosphate backbone.

- DNA polymerase: An enzyme which in the presence of dNTP has the ability to synthesize a complementary copy of a single-stranded DNA template.

- Dideoxynucleoside triphosphate (ddNTP): One OH group of the dNTP is replaced by a hydrogen atom. If a ddNTP is added to a growing strand of DNA the polymerase induced synthesis of new DNA is stopped.

- Primer: A short DNA fragment (15-25 bases) which complements the target strand from which the synthesis of the copy starts. It can carry a radioactive (or other) label. 
The reaction is initiated by a primer and the DNA polymerase starts adding dNTP to one predefined side of the primer. The reaction is carried out in the presence of a low concentration of one type of ddNTP. When a ddNTP molecule is added to the growing strand the DNA polymerase cannot add further bases and the strand growth will stop here. By using ddNTP of the four different types, the polymerase produces partial copies of the original strand which end in a specific type of base. These fragments are then sequenced. The results are obtained by audioradiography using large sheets of X-ray film. Fig. 6.3 shows a schematic outline of an audioradiograph.

\section{The Maxam-Gilbert or chemical sequencing method}

The DNA strand to be sequenced must be radioactively labeled at one end. Chemical reagents which alter one type of base are added. The altered base can then be removed from the sugar-phosphate backbone and the strand is cleaved at the sugar residue lacking the base. This produces DNA fragments of different sizes ending in a specific type of base pair according to the chemical used in step one. The sequencing and detection is analogous to the Sanger method [81].

\subsubsection{Capillary electrophoresis}

Capillary electrophoresis has become increasingly popular as an analysis technique. Compared to other electrophoresis techniques it allows the analysis of small sample volumes and the usage of high voltages which lead to faster separation times [82]. Another advantage is the possibility to use the ability of DNA to absorb UV light for detection, hence no markers are required. A patent for the intrinsic imaging of DNA was filed by Hassard et al. (Imperial College) in 1995 and granted in 1999 [83]. 


\subsubsection{Lambert-Beer's Law}

The absorption of light by a solution with a concentration $c$ is described by LambertBeer's law

$$
I=I_{0} \times 10^{-c k(\lambda) p}
$$

where $I$ is the intensity after the light passed through the sample, $I_{0}$ the initial intensity, $p$ the path length the light travels through the solution and $k(\lambda)$ is the wavelength-dependent absorption coefficient. Fig. 6.4 shows the change in intensity at a wavelength $\lambda=260 \mathrm{~nm}$ as a function of the concentration. Lambert-Beer's law is only valid for low concentrations $(<0.01 \mathrm{~mol} / \mathrm{l})$, before the interaction between the molecules causes an artificial increase in absorption [84]. Typical concentrations of DNA bands to be analyzed are 10-50 ng/ $\mu \mathrm{l}$ where $50 \mathrm{ng} / \mu \mathrm{l}$ single stranded 2000 base-pair DNA corresponds to $\simeq 8 \times 10^{-8} \mathrm{~mol} / \mathrm{l}$.

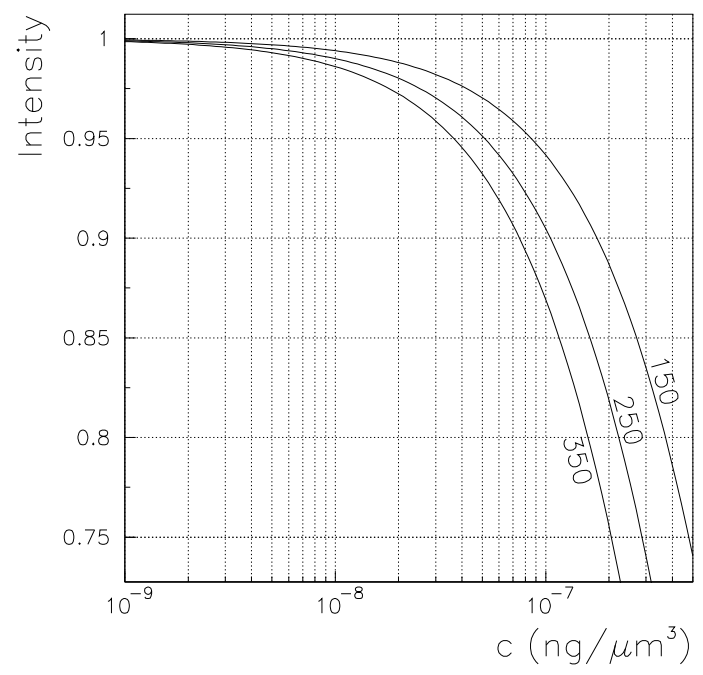

Figure 6.4: Connection between concentration and change in intensity for Lambert-Beer's law for three different path lengths $(150,250$ and $350 \mu \mathrm{m})$. 


\subsection{The $\mu$ DiaGene Project}

\subsubsection{Efficiency of capillary electrophoresis}

Separations are based on differences in electrophoretic mobilities.

A theoretical plate is defined as the distance necessary to achieve complete separation of two fragments and the efficiency of a separation device is often measured in the number of theoretical plates. An efficient separation device has a high number of theoretical plates. In the simplest model [85] the number of theoretical plates $N$ is connected with the electrophoretic mobility $\mu$, the diffusion $D$, the time $t$ and the electrical field $E$ by

$$
N=\frac{\mu^{2} E^{2} t}{2 D}
$$

This means the applied voltage is a determining factor for the efficiency of a device. The voltage is limited by the ability to dissipate the Joule heat that is generated as a result of the electric current passing through the electrophoresis buffer. Cooling is essential as temperature gradients can induce density gradients in the electrophoretic matrix. The electrophoretic mobility $\mu$ of the sample will depend on the temperature. If the average temperature in the sequencing gel exceeds a certain limit, then the sample may disintegrate.

The time $t$ for a solute to migrate through a device of length $L$ with an applied voltage $V$ is

$$
t=\frac{L^{2}}{\mu V}
$$

In order to sequence large amounts of DNA $t$ and therefore $L$ should be small. 


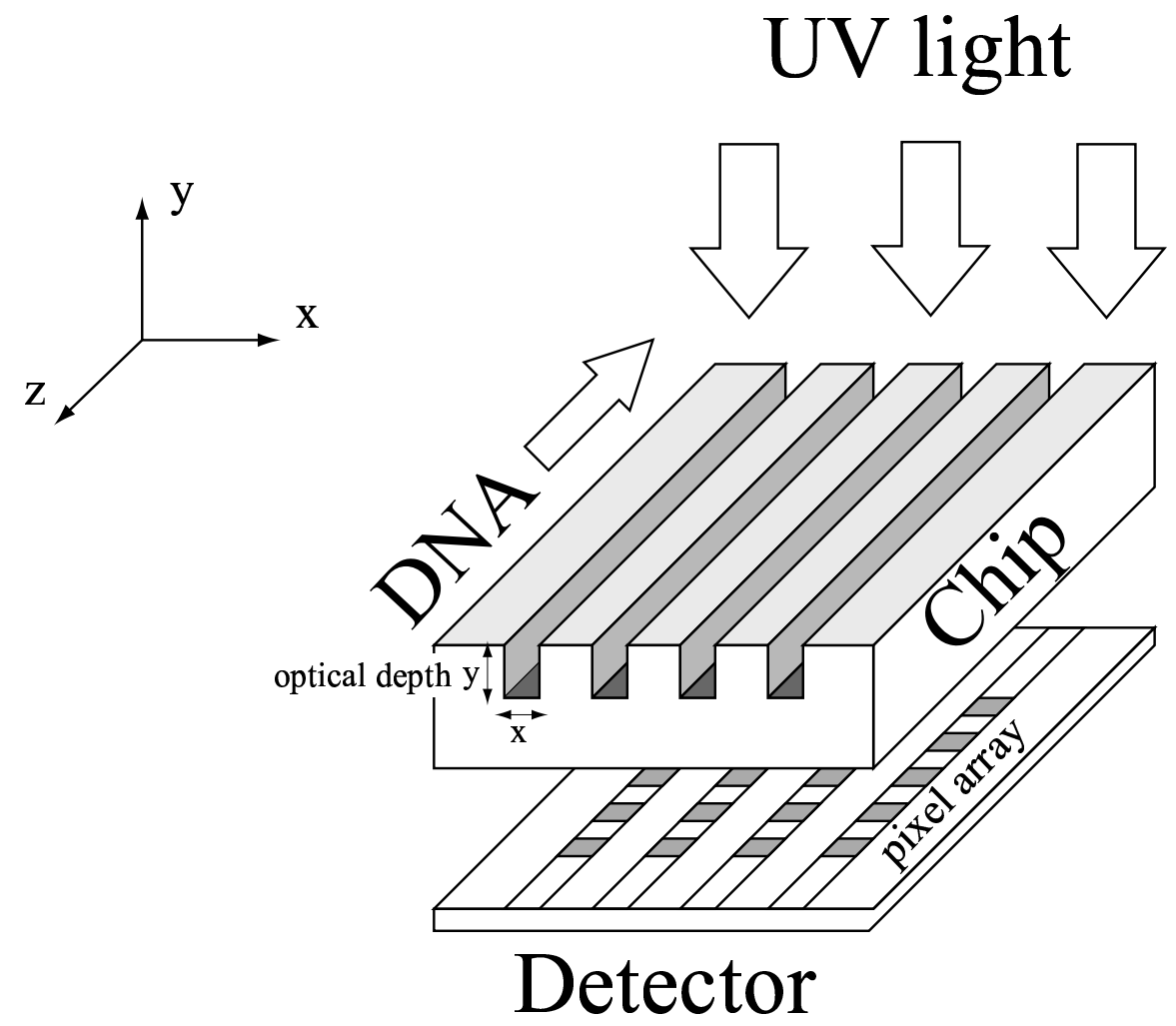

Figure 6.5: $\mu$ DiaGene schematic.

\subsubsection{Status of the $\mu$ DiaGene project}

$\mu$ DiaGene aims at combining several macroscopic technologies at chip ${ }^{1}$ level.

Instead of using capillaries the separation of the fragments will take place in channels etched into a $4 \times 4 \mathrm{~cm}$ wafer. The detection of the DNA fragments in $\mu$ DiaGene is based on the intrinsic absorption of UV light by the DNA molecules. A schematic view of $\mu$ DiaGene is shown in fig. 6.5. The light source will be a laser or a Deuterium lamp combined with a filter. The chip has to be UV transparent and a good heat conductor to allow effective cooling. Several materials for the chip are under investigation:

- Sapphire with channels etched directly into the sapphire.

- Sapphire with a polymer layer on top. The channels are etched into the polymer, so that the channel walls consist of the non-UV transparent polymer and

\footnotetext{
${ }^{1}$ In this context 'chip' refers to a $4 \times 4 \mathrm{~cm}$ wafer (see also fig. 6.5 ).
} 
the channel base is sapphire.

- Polydimethylsiloxane (PDMS) and PDMS coated with nanocrystalline diamond.

A silicon pixel detector is being developed at the University of Cagliari (Italy). As a fallback solution a commercial CCD [86] is being tested at Imperial College.

\subsubsection{Algorithms developed for $\mu$ DiaGene}

A typical measurement in $\mu$ DiaGene consists of several hundred readout periods. This can be used to reduce errors on the measurement or to enhance the signal to noise ratio. Two algorithms adapted from HEP were proposed in the context of $\mu$ DiaGene. They are outlined below. In both cases the velocity of the fragments is assumed to be constant or at least well understood. Detailed descriptions can be found in [87] and [88].

\section{Vertexing Algorithm:}

In each readout period the bands passing the detector appear as a drop in photocurrent. If these minima are plotted in a space-time diagram, background signals will appear as randomly distributed points, while the fragments passing through the area covered by the detector will produce straight lines (see fig. 6.6). The slope of these lines represents the velocity of a fragment. As all the bands start at the same point in time and space these lines will have a common vertex. A fit to this vertex reduces the error on the fit of the velocities. This algorithm is currently used to evaluate measurements made with a test setup [89] at Imperial College. The test stand consists of an agarose filled fused-silica capillary for the electrophoresis and a silicon pixel array for detection. The light source is a Deuterium lamp combined with a filter. The Imperial College group has shown that they can achieve a one basepair resolution in agarose between fragments consisting of up to 400 basepairs. This was a significant step towards a proof of principle for the $\mu$ DiaGene project. 


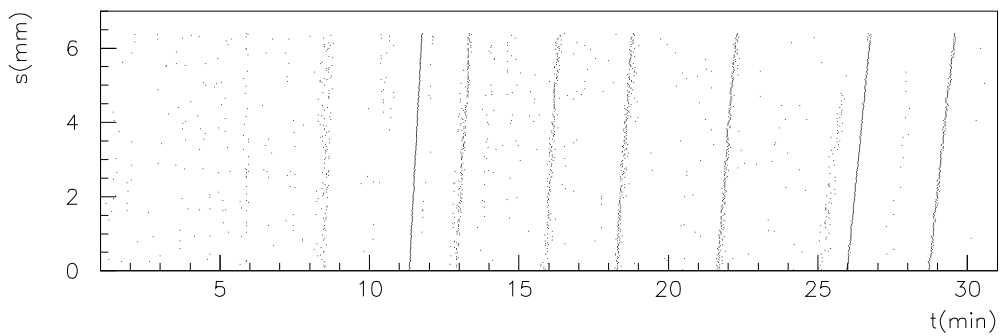

Figure 6.6: Vertexing Algorithm: Space-Time diagram from a capillary measurement at Imperial College [90]. Each point represents a minimum found in one readout period of all the pixels.

\section{Velocity sorting algorithm:}

In contrast to the Vertexing Algorithm, the Velocity Sorting Algorithm does not require detectable minima in the photo-current. Instead, for each readout period the pixels are associated with the velocity a band would have had, if it had reached the pixel at this time. This requires a well-defined starting point for the sample. For each measurement each velocity is associated with a weight. This weight is the difference between an unshadowed photo-current and the measured photo-current. The weighted velocities are then histogrammed. As the noise component is as likely to be positive as it is to be negative compared to the unshadowed current, only the real velocities will be accumulated, while the integrated noise in a given range of velocities grows much more slowly. This leads to an excellent signal to noise ratio (see fig. 6.7).
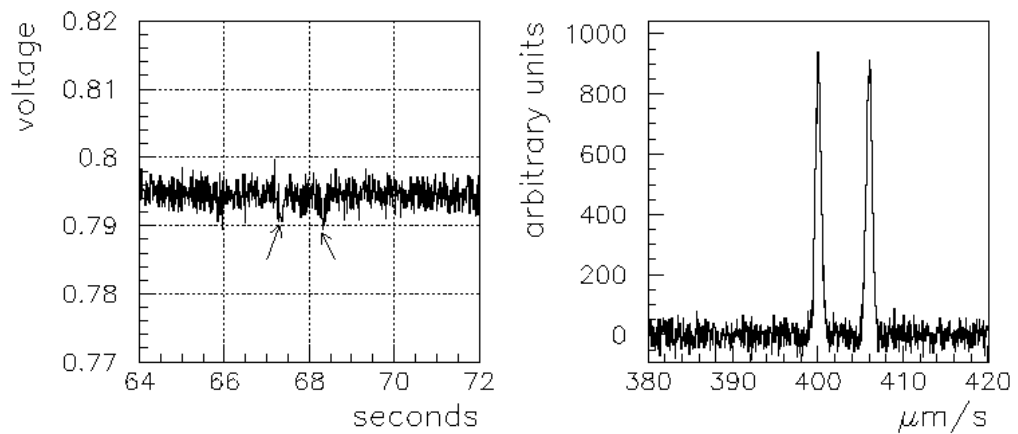

Figure 6.7: Velocity sorting algorithm. The left picture shows a simulated spectrum of one pixel in an array. The signal produced by two bands passing the detector (indicated by the arrows) is of almost the same size as the noise. The right picture shows the reconstructed velocities using the Velocity Sorting Algorithm. The data were simulated using MEDUSA described in section 6.4. Both the signal and the unshadowed photo-current were smeared with random noise for photons and electrons. 


\section{MEDUSA}

\begin{tabular}{|l|}
\hline sample data: \\
velocity \\
fragment length \\
diffusion \\
concentration
\end{tabular}

\section{technical data:} chip detector

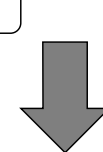

MEDUSA calculates the signal in every pixel for each readout period

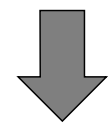

\section{Output formats:}

Raw data (pixel number, voltage)

One pixel over complete runtime

All pixels at a given time

Result of velocity sorting algorithm

Figure 6.8: MEDUSA schematic

\subsection{MEDUSA: A simulation tool for $\mu$ DiaGene}

\subsubsection{MEDUSA Overview}

MEDUSA was developed to investigate the influence of various parameters on the performance of the $\mu$ DiaGene experiment. It also provides Monte Carlo data to test the analysis software. MEDUSA was written in ANSI Standard C++ [91] and should be platform independent. It has been ported to Linux and WindowsNT. All code is my original code, except for the integration routine, which I adapted from [92]. MEDUSA's generic design will also allow its use for similar projects which involve gel electrophoresis in a capillary or on a chip. 
A general outline of the simulation is given in fig. 6.8. MEDUSA reads in the sample and detector data and uses these parameters to calculate the detector response for each readout period. The various input parameters and options are given in section 6.4.4. The results can be displayed using a set of macros which are part of MEDUSA.

\subsubsection{The main program}

Internally each band is represented by an instance of its own class ${ }^{2}$. The band object (class) assembles all the properties (e.g. length, velocity) of a band. It also contains a number of member functions to perform certain actions of a band (move, diffuse) and to obtain derived quantities (current position, concentration in relation to a certain pixel, etc).

An outline of the MEDUSA main program is given below. The steps are in chronological order as performed by the simulation. Loops are described from the outer loops towards the inner ones.

- Detector data are read in and some basic checks are performed.

- The sample data are read in and the absolute amount of DNA (in ng) for each band is determined.

- Runtime loop. For each integration period the actual position and width for each band is calculated. A readout or integration period is the time during which the silicon pixel detector collects charge.

- Loop over all pixels. Performed for each integration period.

- Loop over all bands. For every pixel and integration period the concentration of each band is calculated. The concentrations of all bands are summed up to one concentration seen by the pixel.

- This concentration is then used to determine the absorption seen by the pixel using Lambert-Beer's law.

${ }^{2}$ Different sources define the terms class, object and instance slightly differently. Here an instance is defined as a variable of a class type. An object is the class itself. Unfortunately the terms 'object' and 'instance' are often used interchangeably, therefore adding to the confusion. 
- The data are written to file and can be analyzed using the provided macros or the Vertexing Algorithm [87].

\subsubsection{Algorithm}

At the heart of MEDUSA is the concentration function which returns the concentration for each band for a pixel in a given integration period. The calculation of these concentrations is very $\mathrm{CPU}$ intensive, so the number of calculations should be minimized. In order to achieve reasonable simulation times a number of approximations have been made.

As the concentrations are small, a homogeneous concentration distribution for the area covered by one pixel can be applied. This is justified by the assumption that if $c k p$ is small, Lambert-Beer's law (eq. 6.1) can be approximated as

$$
I=I_{0}\left(1-(c k p \times \ln 10)+\mathcal{O}\left((c k p)^{2}\right)\right) .
$$

Provided the detector response is linear, using an average concentration for the whole area of one pixel therefore results in the same absorption as adding up individual intensities for infinitesimally small parts of a pixel.

The concentration distribution is assumed to be Gaussian. In each integration period the position and the width for each band are calculated. The width is determined by

$$
\sigma=\sqrt{l_{i n j}^{2} / 12+2 \times D \times t}
$$

where $l_{i n j}$ is the injection length, $D$ the diffusion coefficient and $t$ time [93]. The concentration outside of the mean $\pm 3 \sigma$ ( $\simeq 99.73 \%$ of the area of the Gaussian) of each plug is set to zero.

As the concentration distribution broadens with time, the absolute amount of DNA located in the area covered by one pixel has to be calculated individually for each pixel and integration period. A difficulty in using a Gaussian concentration distribu- 
tion is that no analytical solution for the integral exists ${ }^{3}$. To perform the integration MEDUSA uses a routine adapted from [92]. The advantage is that the function to be integrated is a parameter of the routine, so other concentration distributions can be implemented easily.

The movement of the sample plug during an integration period is only negligible if the fragments are slow or the integration times very short. The concentration is calculated at the beginning and end of each integration period and then the average concentration is used to determine the absorption.

It is always possible to ensure that the conditions for these approximations are valid. For large pixels or high velocities an internal division of pixels or integration periods might be necessary.

Once the relevant concentration for a pixel is determined, the change in intensity is calculated using Lambert-Beer's law.

The initial photon intensity (photons $/ \mu \mathrm{m}^{2} / \mathrm{s}$ ) has been estimated from measurements [90] and the spectral distribution of the Deuterium lamp [94]. The number of photons reaching the pixel is smeared with random noise, simulated by Gaussian distributed random numbers $(\sigma=1)$ multiplied by $\sqrt{N_{\text {photons }}}$. The number of electrons collected by a pixel is the number of photons times the quantum efficiency of the detector. It is also smeared with random noise.

\subsubsection{Input data}

The input data are read in from two different files. One file contains the sample data, the other contains technical data like chip geometry and the detector parameters. This allows the comparison of the efficiency of different configurations for a given sample. The sample data are taken from publications (e.g. [95]) or from fits to capillary measurements at Imperial College (fig.6.9(b)). The migration rate of DNA molecules should be to a first approximation inversely proportional to the logarithm

\footnotetext{
${ }^{3}$ The indefinite integral $\int e^{-a x^{2}}$ cannot be evaluated in terms of elementary functions. Only solutions for special cases (e.g. $\int_{0}^{\infty} e^{-a x^{2}}$ ) exist.
} 


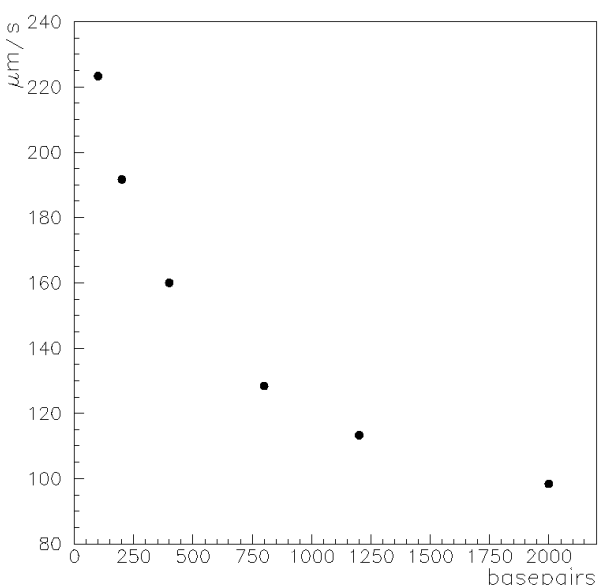

(a) Velocities: Capillary measurement 12.07.99.

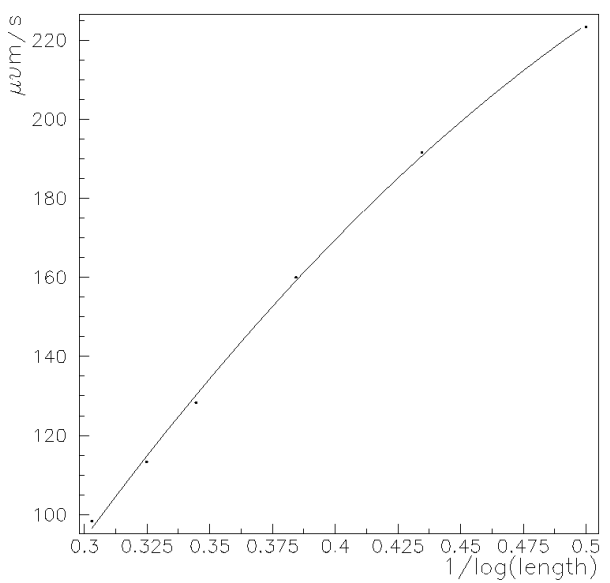

(b) Fit using equation 6.6

Figure 6.9: Fits to measured velocities.

of their molecular weights [81]. MEDUSA uses the function

$$
v=p_{1}+p_{2} \times\left(\frac{1}{\log (l)}\right)+p_{3} \times\left(\frac{1}{\log (l)}\right)^{2}
$$

where $v$ is the velocity of the fragments, $l$ their length in basepairs and $p_{i}$ are fit parameters as this reproduces the original measurements best. The following section gives an overview of the parameters which can be modified. A User Guide for MEDUSA can be found in [96].

\section{Sample data (for each band):}

- velocity in $\mu \mathrm{m} / \mathrm{s}$

- band-length in base-pairs

- diffusion coefficient in $\mu \mathrm{m}^{2} / \mathrm{s}$

- initial concentration in $\mathrm{ng} / \mu \mathrm{m}^{3}$

\section{Technical data:}

- Initial sample plug length in $\mu \mathrm{m}$.

- Number of pixels in $z$. 
- Pixel size in $\mu \mathrm{m}$ in $x$ and in $z$.

- Detector: Average quantum efficiency at a wavelength $240 \mathrm{~nm} \leq \lambda \leq 260$ nm.

- Integration time in seconds.

- Channel width in $x$ and depth in $y$ in $\mu \mathrm{m}$. For a capillary measurement $x$ is set to zero and $y$ is the diameter of the capillary.

- Beginning and end of the instrumented area. Defines the distance between the sample injection point and the location of the detector. Together with the size of the pixel this is used to calculate spacings between the pixels.

- For control purposes the simulation produces an overview of the signal in all pixels at a given time. This time can be set or, if this parameter is zero, MEDUSA will automatically pick a time approximately in the middle of the measurement.

- Pixel number to plot signal in one pixel over the complete runtime. For an example see fig. 6.10. If not set, a pixel in the centre of the array will be used. The data are written to a file and can displayed by using a PAW [97] macro.

- Signal in one pixel without noise (on/off).

- 'Raw data' format: If this parameter is set to zero, the data for each simulated integration period are written to a file. As these files are large $(\approx 125$ MByte $)$ the default setting is 'one', i.e. no data are written out.

- Start of runtime and end of runtime: As the amount of data generated can get quite large, it is often sufficient to generate only data for the time between the first band entering the area covered by the detector and the last band leaving it. If this parameter is set to zero, MEDUSA automatically determines the optimal runtime. 

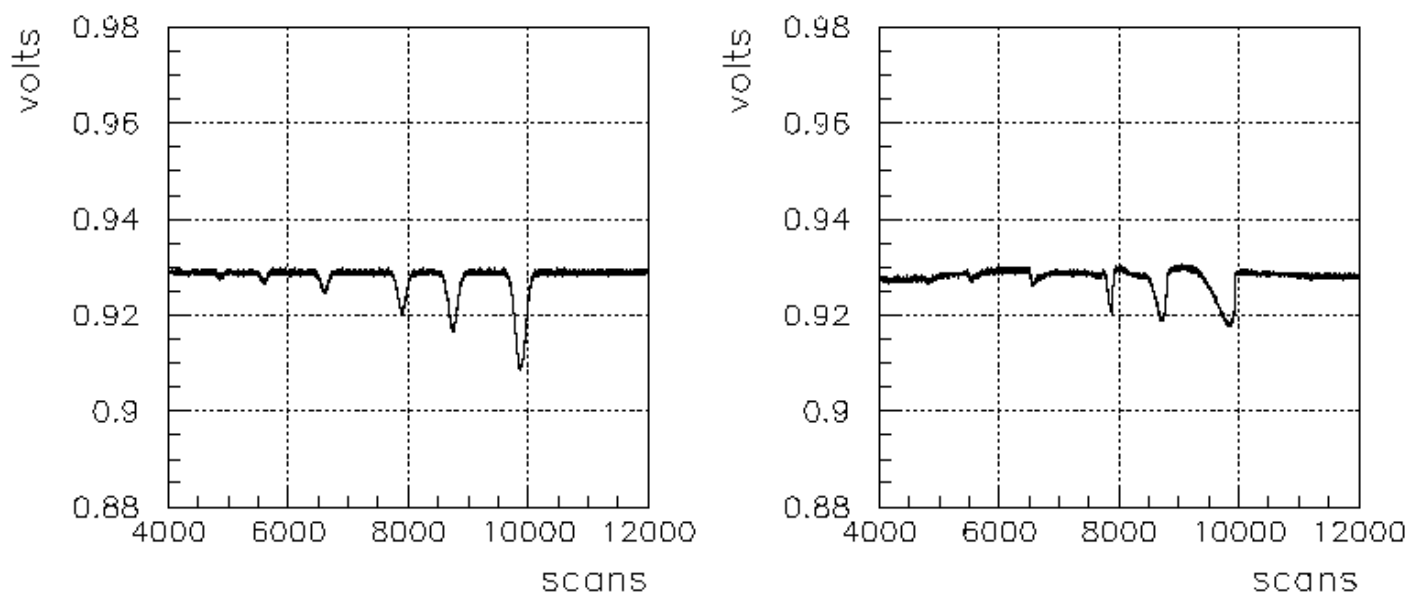

Figure 6.10: Simulation (left) of a capillary measurement and data from a real measurement at Imperial College [90].

\subsubsection{Results}

Fig. 6.10 shows a comparison between a typical capillary measurement using a setup as described in section 6.3.3. The velocities used as input for the simulation were determined using the vertex algorithm [87]. The absorption coefficient for a DNA solution at a wavelength of $260 \mathrm{~nm}$ was measured to be $1740 \mu \mathrm{m}^{2} / \mathrm{ng}$ [98]. For $\mu$ DiaGene the wavelength dependence of the absorption coefficient should be negligible as only light in a narrow band $(254 \mathrm{~nm} \pm 10 \mathrm{~nm})$ is used. The sample was a commercial product [99] composed of an equimolar mixture of six blunt-ended ${ }^{4}$ DNA fragments of 2000, 1200, 800, 400, 200 and 100 base-pairs. The respective concentrations are $50,30,20,10,5$ and $2.5 \mathrm{ng} / \mu \mathrm{l}$. The diameter of the capillary was $500 \mu \mathrm{m}$. The simulation used the same concentrations, but an empirically determined path length of $80 \mu \mathrm{m}$. The results of the simulation were normalized, by using an average voltage of the capillary measurement with no DNA present and the respective value in the simulation as reference points.

\footnotetext{
${ }^{4}$ Blunt-ended DNA (as opposed to sticky ended DNA) is produced such that it cannot stick to other DNA molecules, therefore minimizing the interaction between the fragments.
} 


\section{Conclusion}

Since its initial development in 1999 MEDUSA has been used extensively for different projects within the Imperial College HEP group. It is currently being updated and used to design ultra-high throughput protein analysis systems. 


\section{Chapter 7}

\section{Conclusion}

\subsection{The DØ Run II $b$-physics programme}

The decays presented in this thesis are part of a wider $b$-physics programme at $\mathrm{D} \varnothing$. To put them into context a brief overview of the different areas of $b$-physics investigated at $\mathrm{D} \varnothing$ is given. Most analyses mentioned below are work-in-progress and predictions might change as the understanding of the detector increases. All data are taken from [11] unless otherwise stated.

At end of the chapter the results of this thesis are summarized and future prospects for this work are discussed.

\section{The $\sin 2 \beta$ measurement}

One of the most anticipated measurements is that of $\sin 2 \beta$ in the decay $B_{d}^{0} \rightarrow J / \psi K_{s}^{0}$. Measuring $C P$-violation in this decay requires tagging the flavour of the $B$-meson decaying into the $C P$-eigenstate (cf. section 2.5). As b-quarks are produced in $b \bar{b}$-pairs this can be done either by determining the flavour of the signal $B$-meson directly (same side tagging) or by determining the flavour of the other $B$-meson (opposite side tagging). Same side tagging relies on the correlation between the $B$-meson flavour and the charge of the particles produced in the $b$-quark fragmentation: A $B^{0} \sim(\bar{b} d)$ is likely to have a $\pi^{+} \sim(\bar{d} u)$ nearest in the fragmentation chain, while 
it would be a $\pi^{-}$for a $\overline{B^{0}}$. Opposite side tagging either uses semileptonic decays of $b$-quarks or jet charge tagging. Jet charge tagging exploits the fact that on average the sign of the jet charge is the same as the sign of the $b$-quark producing the jet. In semileptonic decays $\left(b \rightarrow c l^{-} \nu\right)$ the sign of the lepton indicates the flavour of the $b$-quark. Effective tagging algorithms are crucial for the $\sin 2 \beta$ measurement. The quality of a tagging method is described by the effective tagging efficiency $\varepsilon \mathcal{D}^{2}$ where

$$
\varepsilon=\frac{N_{R}+N_{W}}{N} \quad \text { and } \quad \mathcal{D}=\frac{N_{R}-N_{W}}{N_{R}+N_{W}}
$$

with $N$ being the number of reconstructed signal events before tagging, $N_{R}$ the number of correctly tagged events and $N_{W}$ the number of wrong tags. Note that in an ideal case the dilution $\mathcal{D}$ is 1.

For $2 \mathrm{fb}^{-1} \mathrm{D} \varnothing$ expects an event yield of 34000 fully reconstructed $B_{d}^{0} \rightarrow J / \psi K_{s}^{0}$ events in the di-muon mode and a combined tagging efficiency of $10 \%$. This leads to an uncertainty of 0.04 on the $\sin 2 \beta$ measurement. The accuracy of this measurement can be further improved by including $J / \psi \rightarrow e^{+} e^{-}$events to reduce the statistical error.

\section{Lifetime Measurements}

Apart from the lifetime studies presented in this thesis, $D \varnothing$ will attempt to measure the lifetimes of all types of $B$-mesons, including the $B_{c}$ meson. A study similar to this thesis has been done for a lifetime measurement in the channel $B^{+} \rightarrow J / \psi K^{+}$ where $D \varnothing$ expects 12000 fully reconstructed events [100].

In 1998 the CDF collaboration reported the observation [101] of approximately 20 events in the channel $B_{c}^{ \pm} \rightarrow J / \psi l^{ \pm} \nu$. For $2 \mathrm{fb}^{-1} \mathrm{D} \emptyset$ expects about 600 of these events which should be sufficient to improve the $B_{c}$ mass and lifetime measurement. The $b$-physics programme also includes the study of $B$-baryons, especially the $\Lambda_{b}$. 


\section{$B_{s}^{0}$ mixing}

$B_{s}^{0}$ mixing is currently being investigated in the decays $B_{s}^{0} \rightarrow D_{s}^{(*)} \pi, B_{s}^{0} \rightarrow J / \psi K^{* 0}$ and $B_{s}^{0} \rightarrow D_{s}^{(*)} l \nu$. DØ expects to reconstruct 40000 events in the semileptonic decay channels [102]. These decays can only be used for searches up to $\Delta m_{B_{s}}=20 \mathrm{ps}^{-1}$ as the decay length resolution suffers from the undetected neutrino momentum [103]. The hadronic channels have lower statistics (500-1000 events/channel) but a reach of up to $22 \mathrm{ps}^{-1}$ for $2 \mathrm{fb}^{-1}$.

Current experimental results for $\Delta m_{s}$ are $\Delta m_{s} \geq 14.9 \mathrm{ps}^{-1}$ at $95 \%$ confidence level. Theory predicts [104] a value of $15.6 \mathrm{ps}^{-1} \leq \Delta m_{s} \leq 20.5 \mathrm{ps}^{-1}$ at $95 \%$ confidence level under the assumption that there are no contributions from beyond the Standard Model.

\section{Rare Decays}

$\mathrm{D} \varnothing$ has investigated the prospects of measuring the following rare decays: $B_{d}^{0} \rightarrow K^{* 0} \mu^{+} \mu^{-}$: DØ expects between 1300 and 4000 reconstructed events in this decay channel, depending on the cuts used in the analysis. Combined with the expected modest background levels this would be sufficient to establish a signal.

The inclusive decay $b \rightarrow s \mu^{+} \mu^{-}$: While D $\varnothing$ expects to record between 1000 and 2000 events for this measurement, the signal will be swamped by background where the muons come from two different $b$-quarks and $\mathrm{D} \emptyset$ does not expect to be able to establish a signal.

$B_{s}^{0} \rightarrow \mu^{+} \mu^{-}$: D $\varnothing$ will attempt to measure the branching ratio in this channel, but feasibility studies so far have been inconclusive.

\section{The CKM angles $\alpha$ and $\gamma$}

The decay $B_{d}^{0} \rightarrow \pi^{+} \pi^{-}$was initially thought to be an ideal candidate to measure the CKM angle $\alpha$. But measurements [105] by the CLEO experiment indicated that the penguin contribution to this decay is too large for the extraction of $\alpha$. However combining measurements of the decays $B_{d}^{0} \rightarrow \pi^{+} \pi^{-}$and $B_{s}^{0} \rightarrow K^{+} K^{-}$with a $C P$ - 
violation measurement in $B_{d}^{0} \rightarrow J / \psi K_{s}^{0}$ could give access to the angle $\gamma$.

Measuring these channels is difficult as $\mathrm{D} \varnothing$ cannot trigger on the hadronic final states and must rely on the other $B$-meson decaying semileptonically for triggering. Monte Carlo studies have been done and the expected event yields for $2 \mathrm{fb}^{-1}$ of data were found to be 1400 events for $B_{d}^{0} \rightarrow \pi^{+} \pi^{-}, 5600$ events for $B_{d}^{0} \rightarrow K^{+} \pi^{-}$, 2500 events for $B_{s}^{0} \rightarrow K^{+} K^{-}$and 600 events for $B_{s}^{0} \rightarrow K^{+} \pi^{-}$. All these events are automatically flavour tagged due to the trigger requirement. Separating the different decay channels is difficult due to the lack of particle identification. Also it is not possible to separate the two signal decays on the basis of their reconstructed $B$-meson mass as the expected mass resolution of $44 \mathrm{MeV}$ is too large. Furthermore $B_{d}^{0} \rightarrow K^{+} \pi^{-}$lies directly over the two signal channels.

It might be possible to separate the contribution from the different channels with a multivariant fit, but measuring these channels will not be a priority for the time being.

$\mathrm{D} \emptyset$ will also measure the $b \bar{b}$ production cross-section.

\subsection{Summary and Outlook}

After years of preparation, data taking with the D $\varnothing$ Run II detector has begun. Not only has the detector hardware been upgraded, the reconstruction and trigger software has also been completely rewritten to accommodate the higher luminosities and the changes made to the detector. The first results from real data show a good agreement between the expected and the achieved detector performance.

This thesis was written in preparation for Run II and its results are summarized here.

\section{$b$-physics}

While the decays $B_{s}^{0} \rightarrow J / \psi \phi$ and $B_{d}^{0} \rightarrow J / \psi K^{* 0}$ had been studied by CDF during Run I, the absence of a magnetic field prevented $D \varnothing$ from doing a similar analysis at the time. This thesis is the first Run II study of these decays using the full detector simulation and reconstruction. The results show that an improvement of 
the current lifetime measurements in the decays $B_{s}^{0} \rightarrow J / \psi \phi$ and $B_{d}^{0} \rightarrow J / \psi K^{* 0}$ can be achieved with the $\mathrm{D} \varnothing$ detector.

The lifetime measurement is a first step towards further measurements in these channels. An angular analysis allows us to measure the contributions from the different $C P$-eigenstates. The large error on the current measurement [24] is dominated by statistics, so with the comparatively high event yields predicted in this thesis $\mathrm{D} \emptyset$ should be able to increase the accuracy of this measurement in Run II. D $\varnothing$ does not expect to see any $C P$-violation in $B_{s}^{0} \rightarrow J / \psi \phi$, unless there are contributions from beyond the Standard Model. Any $C P$-violation measured by D $\emptyset$ would therefore be a clear indication of New Physics. A simultaneous analysis of the lifetime and angular distributions in $B_{s}^{0} \rightarrow J / \psi \phi$ can give access to the $B_{s}^{0}$ width difference, provided it is sufficiently large. This measurement requires a large number of fully reconstructed decays and will be investigated at end of Run II.

The analyses developed in this thesis make use of large parts of the newly developed $\mathrm{D} \varnothing$ software. This provided a valuable test of the underlying tools (generators, vertexing, etc.) and a number of modifications to them have been made as a result. The successful completion of these analyses show that the software has reached the stage that $\mathrm{D} \emptyset$ is ready to produce physics results.

Experimental data taken at the beginning of Run II have been analysed and a clear signal for the $J / \psi$ meson found. The quality of the signal suffered from the missing matching between the central tracking system and the muon chambers, but this will improve in the future. Reconstructing the $J / \psi$ was a benchmark measurement for a wide range of $b$-physics, including the channels discussed in this thesis.

\section{Trigger software}

In the high-luminosity environment of a $p \bar{p}$ collider fast and reliable triggers are essential. I developed cluster finding software for the SMT and tested it both on Monte Carlo and on real data. The analysis of the cluster resolutions in this thesis helped define the tracking errors, improved the power of the impact parameter tagging and vertex finding which are essential for $b$-physics. The software has been used for a year of data taking without any major problems. 


\section{Simulation of a DNA sequencing device}

Systematic transfer of HEP technologies into other areas and their commercial exploitation will play an important role in the future of particle physics. The simulation developed as part of this thesis was the first proper modelling of a DNA sequencing chip at Imperial College. It has since been heavily used and led to the development of a high throughput DNA sequencing device incorporating a range of HEP technologies and techniques which is currently being commercialised. This is an encouraging result and has lead to further developments in this area. The simulation currently under development for the next generation of devices is based on my original software. 


\section{Appendix A}

\section{A.1 Lorentz transformations}

Using the standard conventions of

$$
\vec{\beta}=\frac{\vec{v}}{c} \quad \gamma=\frac{1}{\sqrt{1-\frac{v^{2}}{c^{2}}}}
$$

the Lorentz transformation can be written as

$$
\begin{aligned}
E^{\prime} & =\gamma(E-\vec{\beta} \vec{p} c) \\
\vec{p}^{\prime} c & =\gamma(-\vec{\beta} E+\vec{p} c)
\end{aligned}
$$

A particle $a$ with four-vector $\left(\frac{E^{a}}{c}, p_{x}^{a}, p_{y}^{a}, p_{z}^{a}\right)$ is to be boosted into the rest-frame of a particle $b$ with $\left(\frac{E^{b}}{c}, p_{x}^{b}, p_{y}^{b}, p_{z}^{b}\right)$. In this rest-frame particle $a$ is now described by the new four-vector $\left(\frac{E^{\prime} a}{c}, p_{x}^{\prime a}, p_{y}^{\prime a}, p_{z}^{\prime a}\right)$. Using the relations $\gamma=\frac{E^{b}}{m_{b}}$ and $\vec{p}=m \gamma \vec{\beta}$ the new vector can be calculated using

$$
\begin{aligned}
E^{\prime a} & =\frac{\left(E^{a} * E^{b}\right)-\left(p_{x}^{a} * p_{x}^{b}+p_{y}^{a} * p_{y}^{b}+p_{z}^{a} * p_{z}^{b}\right)}{m^{b}} \\
\vec{p}^{\prime} a & =\vec{p}^{a}-\frac{E^{\prime a}+E^{a}}{E^{b}+m^{b}} * \vec{p}^{b}
\end{aligned}
$$

The inverse transformation is defined for a particle $r\left(\frac{E^{r}}{c}, p_{x}^{r}, p_{y}^{r}, p_{z}^{r}\right)$ in the restframe of a particle $b$. If particle $b$ moves in another frame with the four-vector 
$\left(\frac{E^{b}}{c}, p_{x}^{b}, p_{y}^{b}, p_{z}^{b}\right)$ the transformation for particle $r$ into this frame is accomplished by

$$
\begin{aligned}
E^{\prime r} & =\frac{\left(E^{r} * E^{b}\right)+\left(p_{x}^{r} * p_{x}^{b}+p_{y}^{r} * p_{y}^{b}+p_{z}^{r} * p_{z}^{b}\right)}{m^{b}} \\
\vec{p}^{\prime r} & =\vec{p}^{r}+\frac{E^{\prime r}+E^{a}}{E^{b}+m^{b}} * \vec{p}^{b}
\end{aligned}
$$

\section{A.2 A cookbook recipe for calculating $\cos \theta_{t}$}

To calculate the transversity angle in the decay $B_{s}^{0} \rightarrow J / \psi\left(\rightarrow \mu^{+} \mu^{-}\right) \phi\left(\rightarrow K^{+} K^{-}\right)$:

1. Boost $K^{+}, K^{-}, \phi$ and $\mu^{+}$from the laboratory frame into the $J / \psi$ rest-frame using the Lorentz transformations as described above.

2. The $\phi$ direction in the $J / \psi$ rest-frame is the $x$-axis.

3. The cross product $K^{-} \times K^{+}$(in this order) is the $z$-axis.

4. $\cos \left(\theta_{t}\right)=\frac{\vec{\mu}^{+} \cdot z \text {-axis }}{\left|\vec{\mu}^{+}\right| \mid z \text {-axis } \mid}$

An equivalent definition for the axes is given by [11]:

$$
\vec{x}=\vec{p}_{\phi}, \quad \vec{y}=\frac{\vec{p}_{K^{+}}-\vec{p}_{\phi}\left(\vec{p}_{\phi} \cdot \vec{p}_{K^{+}}\right)}{\mid \vec{p}_{K^{+}}-\vec{p}_{\phi}\left(\vec{p}_{\phi} \cdot \vec{p}_{K^{+}} \mid\right.}, \quad \vec{z}=\vec{x} \times \vec{z}
$$

where all momentum vectors are unit three-vectors in the $J / \psi$ rest-frame.

\section{A.3 Error on the reconstructed proper decay length}

The proper decay length $L$ is given by

$$
L=\frac{\vec{d} \cdot \vec{p}}{p^{2}} \cdot m_{B}=\frac{d_{x} \cdot p_{x}+d_{y} \cdot p_{y}+d_{z} \cdot p_{z}}{p_{x}^{2}+p_{y}^{2}+p_{z}^{2}} \cdot m_{B}
$$


Differentiating $L$ with respect to $p_{x}$ gives

$$
\begin{aligned}
\frac{\partial L}{\partial p_{x}} & =\frac{d_{x}}{p_{x}^{2}+p_{y}^{2}+p_{z}^{2}} \cdot m_{B}-\frac{2 p_{x}}{\left(p_{x}^{2}+p_{y}^{2}+p_{z}^{2}\right)^{2}} \cdot m_{B} \cdot\left(d_{x} \cdot p_{x}+d_{y} \cdot p_{y}+d_{z} \cdot p_{z}\right) \\
& =\frac{d_{x}}{p^{2}} \cdot m_{B}-\frac{2 p_{x}}{p^{4}} \cdot m_{B} \cdot \vec{d} \cdot \vec{p}
\end{aligned}
$$

and similarly for $\frac{\partial L}{\partial p_{y}}$ and $\frac{\partial L}{\partial p_{z}}$.

Differentiating $L$ with respect to $d_{x}, d_{y}$ and $d_{z}$ yields

$$
\frac{\partial L}{\partial d_{x}}=\frac{p_{x}}{p^{2}} \cdot m_{B} \quad \text { etc. }
$$

and with respect to $m_{B}$

$$
\frac{\partial L}{\partial m_{B}}=\frac{\vec{d} \cdot \vec{p}}{p^{2}}
$$

Combining the above results the error on the decay length $L$ is then

$$
\begin{aligned}
\sigma^{2}(L)=\frac{m_{B}^{2}}{p^{4}}[ & \left(\left(d_{x}, d_{y}, d_{z}\right)-\frac{2 \vec{d} \vec{p}}{p^{2}}\left(p_{x}, p_{y}, p_{z}\right)\right) V_{p}\left(\left(\begin{array}{c}
d_{x} \\
d_{y} \\
d_{z}
\end{array}\right)-\frac{2 \overrightarrow{d \vec{p}}}{p^{2}}\left(\begin{array}{c}
p_{x} \\
p_{y} \\
p_{z}
\end{array}\right)\right) \\
& \left.+\left(p_{x}, p_{y}, p_{z}\right) V_{d}\left(\begin{array}{c}
p_{x} \\
p_{y} \\
p_{z}
\end{array}\right)\right]+\frac{\vec{d} \cdot \vec{p}}{p^{2}} \sigma^{2}\left(m_{B}\right)
\end{aligned}
$$

where $V_{d}$ is the covariance matrix for the decay-vector $\vec{d}$ and $V_{p}$ the covariance matrix for the $B$-meson momentum.

\section{A.4 Straight line fit: Method of the Least Squares}

For a function $f(x ; a)$ which predicts the values $y$ for any $x$ minimising the weighted squared difference between a set of measurements and their predicted values can 
provide an estimate for the parameter $a$. This is known as the method of least squares [106].

When fitting a straight line $y=m x+c$, the sum to be minimised is:

$$
\chi^{2}=\sum_{i} \frac{\left(y_{i}-m x_{i}-c\right)^{2}}{\sigma_{i}^{2}}
$$

where $\sigma_{i}$ is the expected error on the value $y_{i}$. The $x_{i}$ are assumed to be correct. If all the $\sigma_{i}$ are the same they can be taken outside the sum:

$$
\chi^{2}=\frac{1}{\sigma^{2}} \sum_{i}\left(y_{i}-m x_{i}-c\right)^{2}
$$

Differentiating eq. A.12 with respect to $c$ and setting it to zero gives

$$
\frac{d \chi^{2}}{d c}=\frac{1}{\sigma^{2}} \sum_{i}-2\left(y_{i}-\hat{m} x_{i}-\hat{c}\right)=0
$$

which can be reduced to

$$
\bar{y}-\hat{m} \bar{x}-\hat{c}=0
$$

by dividing it by the number of data points $N$. The variables $\hat{m}, \hat{c}$ indicate a fitted value, as opposed to the true value. In a second step, differentiating eq. A.12 with respect to $m$ gives

$$
\sum_{i}-2 x_{i}\left(y_{i}-\hat{m} x_{i}-\hat{c}\right)=0
$$

and again dividing by $N$ results in

$$
\overline{x y}-\hat{m} \overline{x^{2}}-\hat{c} \bar{x}=0
$$


Eliminating $\hat{c}$ from equations A.14 and A.16 gives the result for the slope $\hat{m}$ :

$$
\hat{m}=\frac{\overline{x y}-\bar{x} \bar{y}}{\overline{x^{2}}-\bar{x}^{2}}
$$

\section{Weighted Straight Line Fit}

If the $\sigma_{i}$ are not equal then the simple averages $\overline{x y}, \bar{y}$ etc. in eq. A.14 and A.17 have to be replaced by a weighted average according to

$$
\bar{y}=\frac{\sum_{i=1}^{N} y_{i}}{N} \rightarrow \frac{\sum_{i=1}^{N} y_{i} / \sigma_{i}^{2}}{\sum_{i=1}^{N} 1 / \sigma_{i}^{2}}
$$

\section{A.5 The Method of Maximum Likelihood}

\section{Probability density functions}

In an experiment whose outcome is characterized by a single continuous variable $x$ the probability to observe this variable in the interval $[x, x+d x]$ is $f(x) d x$. The function $f(x)$ is called the probability density function (pdf). It gives the fraction of times that $x$ is observed in the interval $[x, x+d x]$ in the limit of an infinitely large number of observations. It is normalized to one:

$$
\int_{x_{\min }}^{x_{\max }} f(x) d x=1
$$

\section{Maximum likelihood}

For a pdf $f(x \mid \theta)$ where the functional form is known, but which contains at least one unknown parameter $(\theta)$ the method of maximum likelihood can be used to estimate $\theta$ from a finite sample of data [107].

In an experiment with $n$ measurements, the probability for the first measurement to be in the interval $\left[x_{1}, x_{1}+d x_{1}\right]$ is $f\left(x_{1} \mid \theta\right)$, for the second one to be in $\left[x_{2}, x_{2}+d x_{2}\right]$ is $f\left(x_{2} \mid \theta\right)$, etc. 
For $n$ measurements the probability that $x_{i}$ is in $\left[x_{i}, x_{i}+d x_{i}\right]$ is

$$
\prod_{i=1}^{n} f\left(x_{i} \mid \theta\right) d x_{i}
$$

If the pdf and the assumed value for $\theta$ are correct the probability for the measured data should be high. As the $d x_{i}$ do not depend on $\theta$ the same is true for

$$
\mathcal{L}(\theta)=\prod_{i=1}^{n} f\left(x_{i} \mid \theta\right)
$$

$\mathcal{L}$ is called the likelihood function. Provided that $\mathcal{L}$ is differentiable for $\theta$, maximising $\mathcal{L}$ by imposing

$$
\frac{\partial \mathcal{L}}{\partial \theta}=0
$$

will provide an estimator $\hat{\theta}$ for the parameter $\theta$.

Instead of maximising $\mathcal{L}$ it is more common to maximise $\log \mathcal{L}$. As the logarithm is a monotonically increasing function it will be at its maximum for the same $\theta$ as $\mathcal{L}$ would be, but now the product of $\mathcal{L}$ becomes a sum:

$$
\log (\mathcal{L})=\sum_{i=1}^{n} \log f\left(x_{i} \mid \theta\right)
$$

\section{Exponential decay}

The probability density function for an exponential decay with mean lifetime $\lambda$ is

$$
\mathcal{E}(t \mid \lambda)=\frac{1}{\lambda} e^{-\frac{t}{\lambda}}
$$

Therefore

$$
\log \mathcal{E}(t \mid \lambda)=\sum_{i=1}^{n} \log \left(\frac{1}{\lambda} e^{-\frac{t}{\lambda}}\right)=\sum_{i=1}^{n}\left(\log \frac{1}{\lambda}-\frac{t_{i}}{\lambda}\right)
$$


The condition $\frac{\partial \log \mathcal{E}(t \mid \lambda)}{\partial \theta}=0$ then gives the estimator $\hat{\lambda}$ as

$$
\hat{\lambda}=\frac{1}{n} \sum_{i=1}^{n} t_{i}
$$

which is simply the sample mean of the measured time values.

Unlike this example most functions will not be solvable by algebra and it is necessary to use numerical software like MINUIT [108].

All other probability density functions used to fit the $B$-lifetimes in this thesis are listed below:

\section{Gaussian distribution}

The probability density function for a Gaussian distribution with mean $\mu$ and standard deviation $\sigma$ is

$$
\mathcal{G}(x \mid \mu, \sigma)=\frac{1}{\sqrt{2 \pi \sigma^{2}}} e^{-\frac{(x-\mu)^{2}}{2 \sigma^{2}}}
$$

\section{Exponential decay with Gaussian resolution function}

The probability density function of an exponential decay function smeared with a Gaussian resolution function at each point is

$$
\mathcal{F}(x \mid \lambda, \sigma)=\frac{1}{\lambda \sqrt{2 \pi \sigma^{2}}} \int_{0}^{\infty} e^{-u / \lambda} e^{-(u-x)^{2} / 2 \sigma^{2}} d u
$$


The exponential in the integral can be re-written as

$$
\begin{aligned}
-\frac{u}{\lambda}-\frac{(u-x)^{2}}{2 \sigma^{2}} & =-\frac{x^{2}}{2 \sigma^{2}}-\frac{u}{\lambda}+\frac{u x}{\sigma^{2}}-\frac{u^{2}}{2 \sigma^{2}} \\
& =-\frac{x^{2}}{2 \sigma^{2}}-\left(\frac{\left(\sigma^{2}-\lambda x\right) u}{\lambda \sigma^{2}}+\frac{u^{2}}{2 \sigma^{2}}\right) \\
& =-\frac{x^{2}}{2 \sigma^{2}}-\left[\left(\frac{u}{\sqrt{2} \sigma}+\frac{\sigma^{2}-\lambda x}{\sqrt{2} \sigma \lambda}\right)^{2}-\frac{\sigma^{2}}{2 \lambda^{2}}+\frac{x}{\lambda}-\frac{x^{2}}{2 \sigma^{2}}\right] \\
& =-\frac{\left(u+\frac{\sigma^{2}}{\lambda}-x\right)^{2}}{2 \sigma^{2}}+\frac{\sigma^{2}}{2 \lambda^{2}}-\frac{x}{\lambda}
\end{aligned}
$$

Using eq. A.29 the probability density function then becomes

$$
\mathcal{F}(x \mid \lambda, \sigma)=\frac{1}{\lambda \sqrt{2 \pi \sigma^{2}}} e^{-x / \lambda+\sigma^{2} / 2 \lambda^{2}} \int_{0}^{\infty} e^{-\left(u-\left(x-\sigma^{2} / \lambda\right)\right)^{2} / 2 \sigma^{2}} d u
$$

Substituting $\mathrm{k}=\frac{u+\frac{\sigma^{2}}{\lambda}-x}{\sqrt{2} \sigma}$ and $\frac{d k}{d u}=\frac{1}{\sqrt{2} \sigma}$ gives

$$
\mathcal{F}(x \mid \lambda, \sigma)=\frac{1}{2 \lambda} e^{-x / \lambda+\sigma^{2} / 2 \lambda^{2}}\left[1+\operatorname{erf}\left(\left(\frac{x}{\sigma}-\frac{\sigma}{\lambda}\right) / \sqrt{2}\right)\right]
$$

where $\operatorname{erf}(x)$ is the error function

$$
\operatorname{erf}(x)=\frac{2}{\sqrt{\pi}} \int_{0}^{x} e^{-y^{2}} d y
$$

Normalising this function over a restricted range requires the integral $\int_{a}^{b} \mathcal{F}(x \mid \lambda, \sigma)$, which can be obtained by integrating by parts

$$
\begin{aligned}
\int_{a}^{\infty} \mathcal{F}(x \mid \lambda, \sigma) d x= & -\frac{1}{2} e^{-x / \lambda+\sigma^{2} / 2 \lambda^{2}}\left[1+\operatorname{erf}\left(\left(\frac{x}{\sigma}-\frac{\sigma}{\lambda}\right) / \sqrt{2}\right)\right] \\
& +\frac{1}{2} \int e^{-x / \lambda+\frac{1}{2} \sigma^{2} / \lambda^{2}} \frac{2}{\sqrt{\pi}} \frac{1}{\sqrt{2 \sigma^{2}}} e^{-\frac{1}{2}(x / \sigma-\sigma / \lambda)^{2}} d x \\
= & -\lambda \mathcal{F}(x \mid \lambda, \sigma)+\frac{1}{2} \frac{2}{\sqrt{\pi}} \frac{1}{\sqrt{2 \sigma^{2}}} \int e^{-x^{2} / 2 \sigma^{2}} d x \\
= & \frac{1}{2}\left(1+\operatorname{erf}\left(\frac{a}{\sqrt{2 \sigma^{2}}}\right)\right)-\lambda \mathcal{F}(x \mid \lambda, \sigma)
\end{aligned}
$$




\section{Extended maximum likelihood}

Often the number of observations in an experiment (e.g. the number of events found) is itself a Poisson distributed variable with mean $\nu$. The likelihood function can be extended to incorporate this information

$$
\mathcal{L}(\nu, \theta)=\frac{e^{-\nu}}{n !} \nu^{n} \prod_{i=1}^{n} f(x \mid \theta)
$$

This is useful when the overall probability density function is the superposition of $m$ components

$$
f(x \mid \theta)=\sum_{j=1}^{m} \eta_{j} f_{j}(x) \quad \text { and } \quad \sum_{j=1}^{m} \eta_{j}=1
$$

with $\eta_{i}$ being the fractional contribution of each component. Taking the logarithm of equation A.33 gives

$$
\log \mathcal{L}(x \mid \nu, \theta)=-\nu+\sum_{i=1}^{n} \log \left(\sum_{j=1}^{m} \nu \eta_{j} f_{j}(x \mid \theta)\right)
$$

and defining $\mu_{i}=\eta_{i} \nu$ as the expected number of events of type $i$ leads to

$$
\log \mathcal{L}(x \mid \vec{\mu}, \theta)=-\sum_{j=1}^{m} \mu_{j}+\sum_{i=1}^{n} \log \left(\sum_{j=1}^{m} \mu_{j} f_{j}(x \mid \theta)\right)
$$




\section{References}

[1] Atmospheric Neutrino Results From Super Kamiokande and Kamiokande: Evidence for Neutrino Osciallations. Talk given at the 18th International Conference on Neutrino Physics and Astrophysics (NEUTRINO 98), Takayama, Japan, 4-9 Juni 1998. Nucl. Phys. Proc. Suppl. 77(1999) 123-132

[2] M. Peskin, D. Schroeder, An Introduction to Quantum Field Theory, AddisonWesley, 1995

[3] D.E.Groom et al., The European Physical Journal C15(2000)1

[4] C.S. Wu, E. Ambler, R. W. Hayward, D. D. Hoppes, and R. P. Hudson. Phys. Rev. 105, 1413 (1957).

[5] J.H. Christenson, J.W. Cronin, R.Turlay, Phys. Rev. Lett. 13, 138 (1964)

[6] M. Kobayashi, T. Maskawa, Prog. Theor. Phys. 49, 652 (1973)

[7] L. Wolfenstein, Phys. Rev. Lett. 51 (1983) 1945

[8] A.Buras, M. Lautenbacher, G. Ostermaier, Phys. Rev D50 (1994) 3433

[9] P. Ball, R. Fleischer, G.F. Tartarelli, P.Vikas, G. Wilkinson, B decays at the LHC, CERN-TH/2000-101, hep-ph/0003238, March 2000

[10] G. Castelo Branco, L. Lavoura, J.P. Silva, CP Violation, Oxford Science Publications, Oxford 1999 
[11] K. Anikeev et al., B physics at the Tevatron, RunII and beyond, hep-ph/0201071, December 2001

[12] ALEPH collaboration, Phys. Lett. B 486 (2000) 286

[13] KTEV Collaboration, Phys. Rev. Lett. 83, 22, July 1999

[14] NA 48 Collaboration, Phys. Lett. B 465 (1999) 335

[15] BABAR Collaboration (P.F. Harrisonet al.), The BABAR Physics Book, SLACR-504, October 1998

[16] BABAR Collaboration, Improved measurement of the CP violating Asymmetry Amplitude $\sin 2 \beta$, hep-ex/0203007, March 2002

[17] BELLE Collaboration, Improved Measurement of CP Asymmetry in the neutral B-Meson System, hep-ex/0205020, May 2002

[18] E. Fernandez et al., Phys. Rev. Lett. 51 (1983), 1022-1025

[19] N.S. Lockyer et al., Phys. Rev. Lett. 51 (1983), 1316-1319

[20] M. Neubert, Introduction to B physics, hep-ph/0001334

[21] T. Mannel Thirteen Years of Heavy Quark Expansion, Acta Physica Polonica B, No 6, Vol. 32(2001), 1857-1868, April 2001

[22] M. Jacob, G.C. Wick, Annals of Physics 7 (1959), p. 404-428

[23] A.Dighe, I. Dunietz, H. Lipkin, J. Rosner, Phys. Lett. B 369 (1996) p. 144-150

[24] CDF collaboration, Phys. Rev. Lett. 85, 22, p.4668-4673, November 2000

[25] A. Dighe, S. Sen, Phys. Rev. D 59, 074002, February 1999

[26] I. Dunietz, Phys. Rev. D 52, p.3048-3064, 1995

[27] I. Dunietz, R. Fleischer, U. Nierste, In Pursuit of New Physics with $B_{s}$ Decays, FERMILAB-Pub-00/245-T, January 2001 
[28] M. Paulini, B Lifetimes, Mixing and CP Violation at CDF, Int. Journal of Mod. Phys. A14, No. 18, p.2791-2886, July 1999

[29] R. D. Field, The Sources of b-Quarks at the Tevatron and their Correlations, Phys. Rev. D 65(2002), 094009, hep-ph/020112, December 2001

[30] The CDF collaboration, Measurement of b-quark fragmentation fractions in $p \bar{p}$ collisions at $\sqrt{s}=1.8$ TeV, Phys. Rev. Lett. 84, p.1663-1668, February 2001

[31] S. Abachi et al., (DØ Collaboration), Phys.Rev. Lett. 74 (1995) 2632

[32] http://www-bd.fnal.gov/runII/parameters.pdf

[33] S. Abachi et al., (DØ Collaboration), Nuclear Instruments and Methods, A 338, 185 (1994)

[34] The DØ Collaboration, The DØ Upgrade, FERMILAB-PUB-96-357 (1996)

[35] Hal Evans, Results from CDF and DØ, SLAC Topological Workshop, 16 August 2002

[36] The DØ Collaboration, A Silicon Track Trigger for the DØ Experiment, Proposal to Fermilab, DØ Note 3516, September 1998

[37] R. Yarema for the SVXII design group, Beginner's guide to SVXIIe, FermilabTM-1892, June 1994, revised October 1996

[38] M. Roco, The Silicon Microstrip Tracker for the DØ Upgrade, DØ Note 3553, October 1998

[39] DØ Fiber Tracker, Technical Design Report, http://d0server1.fnal.gov/projects/SciFi/cft_home.html, December 1997

[40] D. Lincoln The DØ Central Detector Upgrade, http://d0server1.fnal.gov/ projects/SciFi/vlpc_overview/index.htm, March 2001 
[41] J. Brzezniak et. al, Conceptual Design of a 2 Tesla Superconducting Solenoid for the Fermilab DØ Detector Upgrade, DØ Note 2167, May 1994 http://www-d0.fnal.gov/solenoid

[42] P. Grannis, Preshower Calibration with MIPs, DØ Note 3572, Dec. 1998

[43] V. Büscher, Status of the DØ Detector, Talk given at HCP 2002, Sep 29-Oct 4 2002, Karlsruhe, Germany

[44] G. Alexeev et al., Technical Design Report for the DØ Forward Muon Tracking Detector based on Mini Drift Tubes, DØ Note 3366, December 1997

[45] G. Blazey, The DØ Run II Trigger, Proceedings, Xth Real Time Conference, Beaune, France 1997

[46] A. Boehnlein, G. Brooijmans, D. Claes, M. Souza, Description of DØ L3 Trigger Software Components, DØ Note 3630, April 1999 (http://www-d0.fnal.gov/ 〜moacyr/13/13filter.html)

[47] B. Berliner, CVS II Parallelizing Software Development (http://www.fnal.gov/docs/products/cvs/)

[48] J. Kowalkowski, D. Chakraborty, H. Greenlee, Q. Li, G. Watts, Framework User's Guide (http://www-d0.fnal.gov/run2_offline_software/ framework/framework.html)

[49] Application Software Group, CERN, GEANT Detector Description and Simulation Tool, CERN Program Library Long Writeup W5013, 1993

[50] R. Illingworth, DØ Note 3829, January 2001

[51] D. Bauer, R.Illingworth, DØ Note 3822, January 2001

[52] M. Narain, Talk on numbering of the SMT components http://www-d0.fnal.gov/〜d0upgrade/d0_private/software/silicon/Si_ calalgeo/Si_geo.html, February 1998 
[53] E. Barberis et al., DØ Note 3780, October 2000

[54] code is available under:

http://www-d0.fnal.gov/d0dist/dist/releases/current/simpp/simpp/ SimTkHit.hpp

[55] A. Juste (juste@fnal.gov), private communication, September 2001

[56] A. Juste (juste@fnal.gov) and E. Barberis (barberis@fnal.gov), private communication, June 2001

[57] S. Kulik in a talk given at a DØ meeting, 26. January 2001 http://www-d0.fnal.gov/〜skulik/d0_private/smt/cosmics/

[58] S. Kulik (skulik@fnal.gov) and E. Barberis (barberis@fnal.gov), private communication, February 2001

[59] T. Sjöstrand, Pythia 5.7 and Jetset 7.4 Physics and Manual, December 1998

[60] QQ - the CLEO event generator, http://www.lns.cornell.edu/public/ CLEO/s oft/QQ, unpublished

[61] ROOT An Object Oriented Data Analysis Framework, http://root.cern.ch/, On-going development

[62] A. Ryd, D. Lange, http://www.slac.stanford.edu/ lange/EvtGen/

[63] B. Abbott, email to the $b$-physics group summarizing the Oklahoma Workshop, July 2002

[64] F. Azfar (azfar@fnal.gov), private communication, November 2002

[65] W. Taylor, Prospects for B lifetimes, Oscillations and CP violation at DØ, Talk given at HCP 2002, Sep 29-Oct 4 2002, Karlsruhe, Germany

[66] http://www-d0.fnal.gov/phys_id/muon_id/d0_private/muonid_dh.html, June 2002 
[67] Muon ID Group, Muon ID Version 1.0 (for Winter 2002), http://www-d0. fnal.gov/phys_id/muon_id/d0_private/docs.html, January 2002

[68] A. Schartzman, http://www-d0.fnal.gov/〜sch/kalman/results/results.html, November 2000

[69] http://www-d0.fnal.gov/phys_id/emid/d0_private/conference_ material/welcome.html, July 2002

[70] A. Schwartzman, M. Narain, Primary Vertex Selection, DØ Note 3906, September 2001

[71] CDF Collaboration, Measurement of B Hadron Lifetimes Using $J / \psi$ Final States at CDF, Pys. Rev. D57, 5382, 1998

[72] E. Barberio, B. van Eijk and Z. Was, PHOTOS QED Corrections in Particle Decays, Version 2.2, March 1999

[73] T. Sjöstrand, P. Eden, C. Friberg, L. Lönnblad, G. Miu, S. Mrenna, E. Norrbin, High Energy Physics Event Generation with Pythia 6.1, hep-ph0010017, October 2000

[74] D. Bauer, http://www-d0.fnal.gov/〜dbauer/physics/evtgen/evtgen_at_ d0.html, November 2001

[75] F. Würthwein, http://mit1.fnal.gov/〜fkw/EvtGen/, May 2001

[76] A. Sanchez, private communication, June 2002

[77] http://www-d0.fnal.gov/phys_id/muon_id/d0_private/public_ materials.html, July 2002

[78] J. Watson and F. Crick, Molecular structure of nucleic acids-A structure for deoxyribose nucleic acid, Nature (London) 171, 727-738, 1953 
[79] http://www.accessexcellence.org/AB/GG/ (National Human Genome Research Institute), January 2000

[80] S. Primrose, Principles of Genome Analysis, Blackwell Science Ltd., 95-125, 1998

[81] R. Old and S. Primrose, Principles of Gene Manipulation, Blackwell Science Ltd., 8-9 and 167-177, 1994

[82] C. Culbertson, J. Jorgenson, Lowering the UV Absorbance Detection Limit in Capillary Zone Electrophoresis, Anal. Chem. 1998, 70, 2629-2638

[83] Hassard et al. US patent No. 952032 (filed in 1995, granted in 1999)

[84] D. West, D. Skoog, Fundamentals of Analytical Chemistry, Saunders College Publishing, 1996

[85] P. Grossman, J. Colburn, Capillary Electrophoresis, Academic Press, 1992

[86] Hamamatsu CCD S7030-0907, Technical Data Sheet, 1997

[87] D.Sideris, $\mu$ DiaGene Note \# 5, March 1999

[88] J.Hassard, D.Colling, $\mu$ DiaGene Note \# 4, The velocity sorting algorithm, 1999

[89] A. Mitura, D.Sideris, D. Colling, S. Hassard, J.Hassard, Capillary Electrophoresis, $\mu$ DiaGene Note \# 2, March 1999

[90] D. Sideris (Imperial College), private communication, October 1999

[91] Information Technology - Programming Languages - C++, ISO/IEC C++ Standard, ISO/IEC 14882-1998, September 1998

[92] R. Landau, M. Paez, Computational Physics, Wiley, New York, 1997

[93] C. Effenhauser, A. Manz, M. Widmer, Glass Chips for High Speed Capillary Electrophoresis, Anal. Chem. 1993, 65, 2637-2642, October 1993 
[94] Hamamatsu, Technical Notes TN-102, February 1982 and David Colling (Imperial College), private communication, May 1999

[95] A. Woolley, R. Mathies, Ultra-High-Speed DNA Sequencing using Capillary Electrophoresis Chips, Anal. Chem. 1995, 67, 3676-3680, July 1995

[96] D. Bauer, Status of the MEDUSA simulation tool for the $\mu$ DiaGene project, $\mu$ DiaGene Note \# 14, November 1999

[97] PAW: Physics Analysis Workstation, CERN, http://wwwinfo.cern.ch/asd/paw

[98] D. Colling, private communication, April 1999 (measurement by Prof. Nick Franks (Imperial College))

[99] Life Technologies, Low DNA MASS ${ }^{T M}$ Ladder, Cat. No. 10068-013

[100] R. Illingworth (illingwo@fnal.gov), Development of trigger software for the Silicon and Fibre Trackers and a study of B meson lifetimes for the DØ experiment, Thesis, Imperial College, January 2002

[101] CDF Collaboration, Phys. Rev. Lett. 81, 2432, 1998

[102] R. Gelhaus, B Lifetimes and Mixing at DØ, Talk given at APS meeting in Albuquerque, April 2002

[103] C. Leonidopoulos, B mixing prospects at DØ, Talk given at the CKM workshop at CERN, February 2002

[104] M. Chiuchini et al., 2000 CKM triangle analysis, JHEP 0107 (2001) 013 (hep-ph/0012308), March 2001

[105] CLEO Collaboration, Phys. Rev. Lett. 85, 515, 2000 (hep-ex/0001010)

[106] R.J. Barlow, Statistics, John Wiley \& Sons, p.97-117, 1989

[107] G. Cowan, Statistical Data Analysis, Oxford Science Publications, 1998

[108] F. James, MINUIT, CERN Program Libary Long Writeup D506, August 1998 